\title{
Substituent Effects on Ring Opening of 2-Aziridinylmethyl Radicals
}

\author{
Yi-Min Wang, Yao Fu, Lei Liu, Qing-Xiang Guo \\ Department of Chemistry, University of Science and Technology of China, Hefei 230026, China
}

Table S1. The zero-point correction, thermal correction to Gibbs and electronic energy of $\mathrm{N}$-substituted 2-aziridinylmethyl radicals. S2

Table S2. The zero-point correction, thermal correction to Gibbs and electronic energy of C1-substituted 2-aziridinylmethyl radicals. S3

Table S3. The zero-point correction, thermal correction to Gibbs and electronic energy of Nand C1-disubstituted 2-aziridinylmethyl radicals. S5

Table S4. Delta G of some 2-aziridinylmethyl radicals in organic solutions. S7

Table S5. The zero-point correction, thermal correction to Gibbs and electronic energy of the $\mathrm{C} 1$-substituted cyclopropylcarbinyl and oxiranylcarbinyl radicals. S8

Table S6. The zero-point correction, thermal correction to Gibbs and electronic energy of the bicyclic 2-aziridinylmethyl radicals.

Table S7. The zero-point correction, thermal correction to Gibbs and electronic energy of the bicyclic 2-aziridinylmethyl radicals. S11

Table S8. The zero-point correction, thermal correction to Gibbs and electronic energy of the bicyclic 2-aziridinylmethyl radicals. S12

Table S9. Spin values on C1 of all the radicals in Table S1-S3. S13

Table S10. Spin values on $\mathrm{N}$ of all the radicals in Table S1-S3. . $\mathrm{S} 15$

Table S11. The geometry of all radicals in Table S1-S8 2-aziridinylmethyl radical. ......S17

Table S12. $\hat{\mathrm{S}}^{2}$ values of all the radicals in Table S1-S3.

Table S13. Calculated barriers $\left(\Delta H^{\dagger}\right)$ and reaction enthalpies $(\Delta H)$ for cis- and trans-2-Aziridinylcarbinyl Radical in TableS1.

Table S14. Reaction free energies $(\Delta G)$ for N- and C1-disubstituted 2-aziridinylmethyl radicals.. S119

Possible explanation for the extraordinary $C_{1}$-substituent effects 
Table S1. The zero-point correction, thermal correction to Gibbs and electronic energy of $\mathrm{N}$-substituted 2-aziridinylmethyl radicals. (Unit: Hartree)

\begin{tabular}{|c|c|c|c|}
\hline \multirow[b]{2}{*}{$\mathrm{R}_{1}$} & \multicolumn{3}{|c|}{ Reactants } \\
\hline & $\begin{array}{l}\text { Zero-point } \\
\text { Correction }\end{array}$ & $\begin{array}{c}\text { Thermal Correction to } \\
\text { Gibbs }\end{array}$ & electronic energy \\
\hline$c i s-\mathrm{H}$ & 0.083984 & 0.056977 & -172.1969367 \\
\hline trans $-\mathrm{CH}_{3}$ & 0.111562 & 0.082471 & -211.5155773 \\
\hline trans $-\mathrm{CH}_{2} \mathrm{CH}_{3}$ & 0.14024 & 0.108986 & -250.8452152 \\
\hline cis-CHO & 0.093431 & 0.062991 & -285.5660435 \\
\hline trans $-\mathrm{COCH}_{3}$ & 0.121106 & 0.087694 & -324.9039893 \\
\hline trans $-\mathrm{CONH}_{2}$ & 0.110844 & 0.078024 & -340.9712923 \\
\hline trans $-\mathrm{COOCH}_{3}$ & 0.12691 & 0.092397 & -400.1583359 \\
\hline \multirow[t]{2}{*}{ trans-Ph } & 0.164453 & 0.129745 & -403.3173491 \\
\hline & \multicolumn{3}{|c|}{ Transition States of C-N cleavage } \\
\hline $\mathrm{R}_{1}$ & $\begin{array}{l}\text { Zero-point } \\
\text { Correction }\end{array}$ & $\begin{array}{c}\text { Thermal Correction to } \\
\text { Gibbs } \\
\end{array}$ & electronic energy \\
\hline cis- $\mathrm{H}$ & 0.083286 & 0.056521 & -172.1910725 \\
\hline trans $-\mathrm{CH}_{3}$ & 0.110839 & 0.08187 & -211.5084199 \\
\hline trans $-\mathrm{CH}_{2} \mathrm{CH}_{3}$ & 0.139513 & 0.108384 & -250.8382038 \\
\hline cis-CHO & 0.092985 & 0.062645 & -285.5604858 \\
\hline trans- $\mathrm{COCH}_{3}$ & 0.12068 & 0.08765 & -324.8990871 \\
\hline trans $-\mathrm{CONH}_{2}$ & 0.110537 & 0.078611 & -340.9684511 \\
\hline trans $-\mathrm{COOCH}_{3}$ & 0.126586 & 0.092357 & -400.1530369 \\
\hline \multirow[t]{2}{*}{ trans-Ph } & 0.16382 & 0.129317 & -403.3127288 \\
\hline & \multicolumn{3}{|c|}{ Transition States of C-C cleavage } \\
\hline $\mathrm{R}_{1}$ & $\begin{array}{l}\text { Zero-point } \\
\text { Correction }\end{array}$ & $\begin{array}{c}\text { Thermal Correction to } \\
\text { Gibbs }\end{array}$ & electronic energy \\
\hline cis- $\mathrm{H}$ & 0.082271 & 0.055446 & -172.1793073 \\
\hline trans $-\mathrm{CH}_{3}$ & 0.109986 & 0.081206 & -211.5013504 \\
\hline trans $-\mathrm{CH}_{2} \mathrm{CH}_{3}$ & 0.138554 & 0.107592 & -250.8306054 \\
\hline cis-CHO & 0.091827 & 0.06171 & -285.552699 \\
\hline trans $-\mathrm{COCH}_{3}$ & 0.11957 & 0.086268 & -324.8902918 \\
\hline trans $-\mathrm{CONH}_{2}$ & 0.109416 & 0.077701 & -340.9560055 \\
\hline trans $-\mathrm{COOCH}_{3}$ & 0.125268 & 0.091323 & -400.1440343 \\
\hline trans $-\mathrm{Ph}$ & 0.162929 & 0.128687 & -403.3031555 \\
\hline
\end{tabular}


Table S2. The zero-point correction, thermal correction to Gibbs and electronic energy of C1-substituted 2-aziridinylmethyl radicals. (Unit: Hartree)

\begin{tabular}{|c|c|c|c|}
\hline \multirow[b]{2}{*}{$\mathrm{R}_{2}$} & \multicolumn{3}{|c|}{ Reactants } \\
\hline & $\begin{array}{l}\text { Zero-point } \\
\text { Correction }\end{array}$ & $\begin{array}{c}\text { Thermal Correction to } \\
\text { Gibbs }\end{array}$ & electronic energy \\
\hline cis $-\mathrm{CH}_{3}$ & 0.112221 & 0.083056 & -211.5268917 \\
\hline trans $-\mathrm{CH}_{3}$ & 0.112101 & 0.082958 & -211.5298432 \\
\hline cis-CHO & 0.093676 & 0.063668 & -285.560127 \\
\hline trans $-\mathrm{CHO}$ & 0.093644 & 0.063552 & -285.5622305 \\
\hline$c i s-\mathrm{COCH}_{3}$ & 0.121729 & 0.089158 & -324.8994618 \\
\hline trans $-\mathrm{COCH}_{3}$ & 0.121691 & 0.089028 & -324.9015497 \\
\hline cis-COOH & 0.099473 & 0.067893 & -360.8461628 \\
\hline trans-COOH & 0.099388 & 0.067693 & -360.8477273 \\
\hline cis-CONH 2 & 0.111294 & 0.079267 & -340.9704609 \\
\hline trans-CONH${ }_{2}$ & 0.111496 & 0.079545 & -340.9725093 \\
\hline cis $-\mathrm{CH}=\mathrm{CH}_{2}$ & 0.117002 & 0.086205 & -249.620096 \\
\hline trans $-\mathrm{CH}=\mathrm{CH}_{2}$ & 0.116935 & 0.086171 & -249.6224332 \\
\hline cis- $\mathrm{C} \equiv \mathrm{CH}$ & 0.092936 & 0.062731 & -248.364849 \\
\hline Trans $-\mathrm{C} \equiv \mathrm{CH}$ & 0.092812 & 0.06261 & -248.3650705 \\
\hline cis- $\mathrm{Ph}$ & 0.165216 & 0.130452 & -403.3226916 \\
\hline trans $-\mathrm{Ph}$ & 0.165264 & 0.130412 & -403.3264377 \\
\hline$c i s-\mathrm{F}$ & 0.076194 & 0.047731 & -271.472159 \\
\hline trans $-\mathrm{F}$ & 0.076096 & 0.047458 & -271.472886 \\
\hline cis $-\mathrm{NH}_{2}$ & 0.101378 & 0.07254 & -227.5732409 \\
\hline trans $-\mathrm{NH}_{2}$ & 0.100947 & 0.071905 & -227.5744585 \\
\hline cis- $-\mathrm{OCH}_{3}$ & 0.1169 & 0.08597 & -286.7617419 \\
\hline \multirow[t]{2}{*}{ trans $-\mathrm{OCH}_{3}$} & 0.116692 & 0.085516 & -286.7626756 \\
\hline & \multicolumn{3}{|c|}{ Transition States of C-N cleavage } \\
\hline $\mathrm{R}_{2}$ & $\begin{array}{l}\text { Zero-point } \\
\text { Correction }\end{array}$ & $\begin{array}{c}\text { Thermal Correction to } \\
\text { Gibbs } \\
\end{array}$ & electronic energy \\
\hline cis- $\mathrm{CH}_{3}$ & 0.111652 & 0.082909 & -211.5217851 \\
\hline trans $-\mathrm{CH}_{3}$ & 0.111434 & 0.082644 & -211.5241279 \\
\hline cis-CHO & 0.092757 & 0.062765 & -285.5499787 \\
\hline trans-CHO & 0.092382 & 0.062161 & -285.5490201 \\
\hline cis- $\mathrm{COCH}_{3}$ & 0.120939 & 0.088544 & -324.8900542 \\
\hline trans $-\mathrm{COCH}_{3}$ & 0.120621 & 0.088049 & -324.8892838 \\
\hline cis-COOH & 0.098711 & 0.0672 & -360.8371266 \\
\hline trans $-\mathrm{COOH}$ & 0.098311 & 0.066517 & -360.8360787 \\
\hline cis-CONH${ }_{2}$ & 0.110475 & 0.07847 & -340.9619362 \\
\hline trans- $\mathrm{CONH}_{2}$ & 0.110655 & 0.078877 & -340.9649445 \\
\hline cis $-\mathrm{CH}=\mathrm{CH}_{2}$ & 0.116384 & 0.085901 & -249.6126439 \\
\hline
\end{tabular}




\begin{tabular}{|c|c|c|c|}
\hline trans $-\mathrm{CH}=\mathrm{CH}_{2}$ & 0.116228 & 0.085721 & -249.6143652 \\
\hline cis $-\mathrm{C} \equiv \mathrm{CH}$ & 0.092346 & 0.062473 & -248.3574208 \\
\hline Trans $-\mathrm{C} \equiv \mathrm{CH}$ & 0.091884 & 0.061833 & -248.3553073 \\
\hline cis-Ph & 0.164383 & 0.129799 & -403.3142411 \\
\hline trans $-\mathrm{Ph}$ & 0.164549 & 0.129791 & -403.3184551 \\
\hline cis-F & 0.075535 & 0.047298 & -271.470174 \\
\hline trans $-\mathrm{F}$ & 0.075385 & 0.047071 & -271.4690869 \\
\hline cis $-\mathrm{NH}_{2}$ & 0.10072 & 0.072165 & -227.5686511 \\
\hline trans $-\mathrm{NH}_{2}$ & 0.100344 & 0.071682 & -227.5693949 \\
\hline cis $-\mathrm{OCH}_{3}$ & 0.116246 & 0.085578 & -286.7582967 \\
\hline trans $-\mathrm{OCH}_{3}$ & 0.116 & 0.085236 & -286.7579303 \\
\hline & \multicolumn{3}{|c|}{ Transition States of C-C cleavage } \\
\hline $\mathrm{R}_{2}$ & $\begin{array}{l}\text { Zero-point } \\
\text { Correction }\end{array}$ & $\begin{array}{c}\text { Thermal Correction to } \\
\text { Gibbs }\end{array}$ & electronic energy \\
\hline cis $-\mathrm{CH}_{3}$ & 0.110858 & 0.08175 & -211.5125784 \\
\hline trans $-\mathrm{CH}_{3}$ & 0.110654 & 0.081399 & -211.5134171 \\
\hline cis-CHO & 0.093056 & 0.063286 & -285.5568806 \\
\hline trans-CHO & 0.093209 & 0.063342 & -285.5587946 \\
\hline cis- $\mathrm{COCH}_{3}$ & 0.12093 & 0.08781 & -324.8945114 \\
\hline trans- $\mathrm{COCH}_{3}$ & 0.1211 & 0.088254 & -324.8963358 \\
\hline cis-COOH & 0.098644 & 0.067342 & -360.8393558 \\
\hline trans-COOH & 0.098681 & 0.067237 & -360.8405673 \\
\hline cis- $\mathrm{CONH}_{2}$ & 0.110521 & 0.078908 & -340.9622571 \\
\hline trans- $\mathrm{CONH}_{2}$ & 0.110768 & 0.079184 & -340.9619588 \\
\hline cis $-\mathrm{CH}=\mathrm{CH}_{2}$ & 0.116041 & 0.085715 & -249.6133238 \\
\hline trans $-\mathrm{CH}=\mathrm{CH}_{2}$ & 0.11601 & 0.085681 & -249.6164058 \\
\hline cis $-\mathrm{C} \equiv \mathrm{CH}$ & 0.091794 & 0.061752 & -248.3571444 \\
\hline Trans $-\mathrm{C} \equiv \mathrm{CH}$ & 0.091814 & 0.061763 & -248.3585827 \\
\hline cis-Ph & 0.164297 & 0.130027 & -403.3164524 \\
\hline trans $-\mathrm{Ph}$ & 0.16448 & 0.130213 & -403.3200075 \\
\hline$c i s-\mathrm{F}$ & 0.074834 & 0.046296 & -271.45421 \\
\hline trans $-\mathrm{F}$ & 0.074981 & 0.046505 & -271.4558543 \\
\hline$c i s-\mathrm{NH}_{2}$ & 0.099601 & 0.070718 & -227.5586298 \\
\hline trans $-\mathrm{NH}_{2}$ & 0.099455 & 0.070708 & -227.5639789 \\
\hline cis- $\mathrm{OCH}_{3}$ & 0.115697 & 0.084591 & -286.749379 \\
\hline trans $-\mathrm{OCH}_{3}$ & 0.11578 & 0.084431 & -286.7487993 \\
\hline
\end{tabular}


Table S3. The zero-point correction, thermal correction to Gibbs and electronic energy of Nand $\mathrm{C} 1$-disubstituted 2-aziridinylmethyl radicals. (Unit: Hartree)

\begin{tabular}{|c|c|c|c|c|}
\hline \multirow[b]{2}{*}{$\mathrm{R}_{1}$} & \multirow[b]{2}{*}{$\mathrm{R}_{2}$} & \multicolumn{3}{|c|}{ Reactants } \\
\hline & & $\begin{array}{l}\text { Zero-point } \\
\text { Correction }\end{array}$ & $\begin{array}{c}\text { Thermal Correction to } \\
\text { Gibbs }\end{array}$ & electronic energy \\
\hline trans $-\mathrm{CH}_{3}$ & cis- $-\mathrm{CH}_{3}$ & 0.112221 & 0.083056 & -211.5268917 \\
\hline cis $-\mathrm{CH}_{3}$ & trans $-\mathrm{CH}_{3}$ & 0.112101 & 0.082958 & -211.5298432 \\
\hline trans $-\mathrm{CH}_{3}$ & cis-CHO & 0.139651 & 0.108478 & -250.8469908 \\
\hline cis $-\mathrm{CH}_{3}$ & trans-CHO & 0.139874 & 0.108734 & -250.8457521 \\
\hline trans $-\mathrm{CH}_{3}$ & $c i s-\mathrm{Ph}$ & 0.121413 & 0.088888 & -324.8987453 \\
\hline cis $-\mathrm{CH}_{3}$ & trans $-\mathrm{Ph}$ & 0.121483 & 0.089038 & -324.8993857 \\
\hline trans-CHO & cis $-\mathrm{CH}_{3}$ & 0.192467 & 0.155775 & -442.6490375 \\
\hline cis-CHO & trans $-\mathrm{CH}_{3}$ & 0.192606 & 0.156231 & -442.6484756 \\
\hline trans-CHO & cis-CHO & 0.093676 & 0.063668 & -285.560127 \\
\hline cis-CHO & trans-CHO & 0.093644 & 0.063552 & -285.5622305 \\
\hline trans-CHO & cis-Ph & 0.120703 & 0.088279 & -324.8776713 \\
\hline cis-CHO & trans $-\mathrm{Ph}$ & 0.120962 & 0.08857 & -324.8770193 \\
\hline trans $-\mathrm{Ph}$ & cis $-\mathrm{CH}_{3}$ & 0.102213 & 0.068393 & -398.9243973 \\
\hline cis $-\mathrm{Ph}$ & trans $-\mathrm{CH}_{3}$ & 0.102376 & 0.068624 & -398.9258987 \\
\hline trans $-\mathrm{Ph}$ & cis-CHO & 0.173452 & 0.135562 & -516.6782297 \\
\hline cis-Ph & trans-CHO & 0.173584 & 0.135887 & -516.6781757 \\
\hline trans $-\mathrm{Ph}$ & cis-Ph & 0.165216 & 0.130452 & -403.3226916 \\
\hline \multirow[t]{2}{*}{ cis-Ph } & trans $-\mathrm{Ph}$ & 0.165264 & 0.130412 & -403.3264377 \\
\hline & & \multicolumn{3}{|c|}{ Transition States of C-N cleavage } \\
\hline $\mathrm{R}_{1}$ & $\mathrm{R}_{2}$ & $\begin{array}{l}\text { Zero-point } \\
\text { Correction }\end{array}$ & $\begin{array}{c}\text { Thermal Correction to } \\
\text { Gibbs }\end{array}$ & electronic energy \\
\hline trans $-\mathrm{CH}_{3}$ & cis- $-\mathrm{CH}_{3}$ & 0.111652 & 0.082909 & -211.5217851 \\
\hline cis- $\mathrm{CH}_{3}$ & trans $-\mathrm{CH}_{3}$ & 0.111434 & 0.082644 & -211.5241279 \\
\hline trans $-\mathrm{CH}_{3}$ & cis-CHO & 0.138979 & 0.108041 & -250.8397921 \\
\hline cis- $\mathrm{CH}_{3}$ & trans-CHO & 0.139129 & 0.108136 & -250.8417272 \\
\hline trans $-\mathrm{CH}_{3}$ & cis-Ph & 0.120937 & 0.08854 & -324.89264 \\
\hline cis $-\mathrm{CH}_{3}$ & trans $-\mathrm{Ph}$ & 0.121046 & 0.088729 & -324.8934755 \\
\hline trans-CHO & cis- $\mathrm{CH}_{3}$ & 0.191929 & 0.155623 & -442.6442136 \\
\hline cis-CHO & trans $-\mathrm{CH}_{3}$ & 0.192094 & 0.155997 & -442.6459517 \\
\hline trans-CHO & cis-CHO & 0.092757 & 0.062765 & -285.5499787 \\
\hline cis-CHO & trans-CHO & 0.092382 & 0.062161 & -285.5490201 \\
\hline trans-CHO & cis-Ph & 0.119775 & 0.087151 & -324.8656159 \\
\hline cis-CHO & trans $-\mathrm{Ph}$ & 0.119993 & 0.087315 & -324.8685365 \\
\hline trans $-\mathrm{Ph}$ & cis- $-\mathrm{CH}_{3}$ & 0.101639 & 0.06762 & -398.9154899 \\
\hline cis- $\mathrm{Ph}$ & trans $-\mathrm{CH}_{3}$ & 0.101744 & 0.067703 & -398.9169979 \\
\hline
\end{tabular}




\begin{tabular}{|c|c|c|c|c|}
\hline trans $-\mathrm{Ph}$ & cis-CHO & 0.172683 & 0.134584 & -516.6697567 \\
\hline cis- $\mathrm{Ph}$ & trans-CHO & 0.172934 & 0.135146 & -516.672261 \\
\hline trans-Ph & cis-Ph & 0.164383 & 0.129799 & -403.3142411 \\
\hline$c i s-\mathrm{Ph}$ & trans $-\mathrm{Ph}$ & 0.164549 & 0.129791 & -403.3184551 \\
\hline & & \multicolumn{3}{|c|}{ Transition States of C-C cleavage } \\
\hline $\mathrm{R}_{1}$ & $\mathrm{R}_{2}$ & $\begin{array}{l}\text { Zero-point } \\
\text { Correction }\end{array}$ & $\begin{array}{l}\text { Thermal Correction to } \\
\text { Gibbs }\end{array}$ & electronic energy \\
\hline trans $-\mathrm{CH}_{3}$ & cis- $-\mathrm{CH}_{3}$ & 0.110858 & 0.08175 & -211.5125784 \\
\hline cis $-\mathrm{CH}_{3}$ & trans $-\mathrm{CH}_{3}$ & 0.110654 & 0.081399 & -211.5134171 \\
\hline trans $-\mathrm{CH}_{3}$ & cis-CHO & 0.138298 & 0.10712 & -250.8350943 \\
\hline cis $-\mathrm{CH}_{3}$ & trans-CHO & 0.138646 & 0.107641 & -250.8326384 \\
\hline trans $-\mathrm{CH}_{3}$ & cis-Ph & 0.120096 & 0.087637 & -324.8879872 \\
\hline cis $-\mathrm{CH}_{3}$ & trans $-\mathrm{Ph}$ & 0.120196 & 0.087826 & -324.8875484 \\
\hline trans-CHO & cis- $-\mathrm{CH}_{3}$ & 0.191179 & 0.154735 & -442.6370681 \\
\hline cis-CHO & trans $-\mathrm{CH}_{3}$ & 0.191176 & 0.154758 & -442.6337777 \\
\hline trans-CHO & cis-CHO & 0.093056 & 0.063286 & -285.5568806 \\
\hline cis-CHO & trans-CHO & 0.093209 & 0.063342 & -285.5587946 \\
\hline trans-CHO & $c i s-\mathrm{Ph}$ & 0.120101 & 0.088061 & -324.8735608 \\
\hline cis-CHO & trans- $\mathrm{Ph}$ & 0.12047 & 0.088537 & -324.8734998 \\
\hline trans $-\mathrm{Ph}$ & cis $-\mathrm{CH}_{3}$ & 0.101475 & 0.068034 & -398.9204144 \\
\hline$c i s-\mathrm{Ph}$ & trans $-\mathrm{CH}_{3}$ & 0.101741 & 0.068402 & -398.9223136 \\
\hline trans-Ph & cis-CHO & 0.172781 & 0.135362 & -516.6741317 \\
\hline cis-Ph & trans-CHO & 0.173061 & 0.135845 & -516.673673 \\
\hline trans $-\mathrm{Ph}$ & $c i s-\mathrm{Ph}$ & 0.164297 & 0.130027 & -403.3164524 \\
\hline cis-Ph & trans-Ph & 0.16448 & 0.130213 & -403.3200075 \\
\hline
\end{tabular}


Table S4. Delta G of some 2-aziridinylmethyl radicals in organic solutions. (Unit: kcal)

\begin{tabular}{cccccc}
\hline & & \multicolumn{5}{c}{ Reactants } \\
\cline { 3 - 6 } $\mathrm{R}_{2}$ & $\mathrm{R}_{1}$ & $\mathrm{C}_{6} \mathrm{H}_{12}$ & THF & MeCN & DMSO \\
\cline { 3 - 6 } trans $-\mathrm{CH}_{3}$ & cis $-\mathrm{CH}_{3}$ & 3.4 & 4.39 & 5.78 & 4.38 \\
trans $-\mathrm{CHO}$ & cis- $\mathrm{CHO}$ & -1.33 & -4.15 & -3.87 & -5.82 \\
cis $-\mathrm{Ph}$ & trans $-\mathrm{Ph}$ & 0.84 & 1 & 3.54 & 0 \\
\hline & & \multicolumn{4}{c}{ Transition States of C-N cleavage } \\
\cline { 3 - 6 } $\mathrm{R}_{2}$ & $\mathrm{R}_{1}$ & $\mathrm{C}_{6} \mathrm{H}_{12}$ & THF & MeCN & DMSO \\
\cline { 3 - 6 } trans $-\mathrm{CH}_{3}$ & cis $-\mathrm{CH}_{3}$ & 3.16 & 3.75 & 4.99 & 3.55 \\
trans $-\mathrm{CHO}$ & cis $-\mathrm{CHO}$ & -1.18 & -3.68 & -3.23 & -5.18 \\
cis $-\mathrm{Ph}$ & trans $-\mathrm{Ph}$ & 0.91 & 0.89 & 3.35 & -0.14 \\
\hline & & \multicolumn{4}{c}{ Transition States of C-C cleavage } \\
\cline { 3 - 6 } $\mathrm{R}_{2}$ & $\mathrm{R}_{1}$ & $\mathrm{C}_{6} \mathrm{H}_{12}$ & THF & MeCN & DMSO \\
\cline { 3 - 6 } trans $-\mathrm{CH} \mathrm{H}_{3}$ & cis $-\mathrm{CH} \mathrm{H}_{3}$ & 3.52 & 4.63 & 6.1 & 4.69 \\
trans $-\mathrm{CHO}$ & cis $-\mathrm{CHO}$ & -1.54 & -4.73 & -4.62 & -6.58 \\
cis $-\mathrm{Ph}$ & trans $-\mathrm{Ph}$ & 0.92 & 1.18 & 3.84 & 0.28 \\
\hline
\end{tabular}


Table S5. The zero-point correction, thermal correction to Gibbs and electronic energy of the $\mathrm{C} 1$-substituted cyclopropylcarbinyl and oxiranylcarbinyl radicals. (Unit: Hartree)

\begin{tabular}{|c|c|c|c|c|}
\hline \multirow[b]{2}{*}{$X$} & \multirow[b]{2}{*}{$\mathrm{R}$} & \multicolumn{3}{|c|}{ Reactants } \\
\hline & & $\begin{array}{l}\text { Zero-point } \\
\text { Correction }\end{array}$ & $\begin{array}{c}\text { Thermal Correction to } \\
\text { Gibbs }\end{array}$ & electronic energy \\
\hline \multirow[t]{5}{*}{$\mathrm{CH}_{2}$} & $\mathrm{H}$ & 0.094945 & 0.067463 & -156.1434772 \\
\hline & cis-CHO & 0.104908 & 0.074649 & -269.5063766 \\
\hline & trans-CHO & 0.104878 & 0.074459 & -269.5092869 \\
\hline & cis-Ph & 0.176503 & 0.140773 & -387.2669565 \\
\hline & trans $-\mathrm{Ph}$ & 0.176608 & 0.141651 & -387.2701808 \\
\hline \multirow[t]{6}{*}{$\mathrm{O}$} & $\mathrm{H}$ & 0.07102 & 0.044018 & -192.0460601 \\
\hline & cis-CHO & 0.08022 & 0.049777 & -305.4054533 \\
\hline & trans-CHO & 0.080272 & 0.04987 & -305.4080972 \\
\hline & cis- $\mathrm{Ph}$ & 0.152605 & 0.118066 & -423.1749768 \\
\hline & trans $-\mathrm{Ph}$ & 0.15246 & 0.117824 & -423.1772738 \\
\hline & & \multicolumn{3}{|c|}{ Transition States of C-N cleavage } \\
\hline$X$ & $\mathrm{R}$ & $\begin{array}{l}\text { Zero-point } \\
\text { Correction } \\
\end{array}$ & $\begin{array}{c}\text { Thermal Correction to } \\
\text { Gibbs }\end{array}$ & electronic energy \\
\hline \multirow[t]{5}{*}{$\mathrm{CH}_{2}$} & $\mathrm{H}$ & 0.093818 & 0.066855 & -156.1286291 \\
\hline & cis-CHO & 0.103105 & 0.072667 & -269.4851607 \\
\hline & trans-CHO & 0.103075 & 0.072516 & -269.487478 \\
\hline & cis- $\mathrm{Ph}$ & 0.175306 & 0.140715 & -387.2511753 \\
\hline & trans-Ph & 0.175093 & 0.140153 & -387.2532198 \\
\hline \multirow[t]{6}{*}{$\mathrm{O}$} & $\mathrm{H}$ & 0.070811 & 0.04407 & -192.0395213 \\
\hline & cis-CHO & 0.079823 & 0.049371 & -305.3961749 \\
\hline & trans-CHO & 0.079822 & 0.049391 & -305.3986793 \\
\hline & cis- $\mathrm{Ph}$ & 0.152305 & 0.117998 & -423.1679428 \\
\hline & trans $-\mathrm{Ph}$ & 0.152112 & 0.117515 & -423.1688741 \\
\hline & & \multicolumn{3}{|c|}{ Transition States of C-C cleavage } \\
\hline $\mathrm{X}$ & $\mathrm{R}$ & $\begin{array}{l}\text { Zero-point } \\
\text { Correction }\end{array}$ & $\begin{array}{c}\text { Thermal Correction to } \\
\text { Gibbs }\end{array}$ & electronic energy \\
\hline \multirow[t]{5}{*}{$\mathrm{CH}_{2}$} & $\mathrm{H}$ & 0.093817 & 0.066853 & -156.12863 \\
\hline & cis-CHO & 0.104449 & 0.074465 & -269.5034998 \\
\hline & trans-CHO & 0.10458 & 0.074489 & -269.5063763 \\
\hline & cis- $\mathrm{Ph}$ & 0.176012 & 0.141605 & -387.2623737 \\
\hline & trans $-\mathrm{Ph}$ & 0.175997 & 0.141507 & -387.2656065 \\
\hline \multirow[t]{5}{*}{$\mathrm{O}$} & $\mathrm{H}$ & 0.069048 & 0.042236 & -192.024619 \\
\hline & cis-CHO & 0.079309 & 0.04919 & -305.396394 \\
\hline & trans-CHO & 0.079491 & 0.049404 & -305.398671 \\
\hline & cis- $\mathrm{Ph}$ & 0.151369 & 0.117235 & -423.1648715 \\
\hline & trans $-\mathrm{Ph}$ & 0.151459 & 0.117268 & -423.1674839 \\
\hline
\end{tabular}


Table S6. The zero-point correction, thermal correction to Gibbs and electronic energy of the bicyclic 2-aziridinylmethyl radicals. (Unit: Hartree)

\begin{tabular}{|c|c|c|c|c|}
\hline \multirow[b]{2}{*}{$\mathrm{N}$} & \multirow[b]{2}{*}{ Structure } & \multicolumn{3}{|c|}{ Reactants } \\
\hline & & $\begin{array}{l}\text { Zero-point } \\
\text { Correction }\end{array}$ & $\begin{array}{c}\text { Thermal Correction } \\
\text { to Gibbs } \\
\end{array}$ & electronic energy \\
\hline 1-trans & & 0.088881 & 0.060892 & -210.1229104 \\
\hline 1-cis & & 0.088707 & 0.060687 & -210.1264827 \\
\hline 2-trans & & 0.118781 & 0.089518 & -249.5984639 \\
\hline 2-cis & & 0.118296 & 0.088738 & -249.6005855 \\
\hline 3-trans & & 0.148758 & 0.117888 & -288.9569799 \\
\hline 3-cis & & 0.14853 & 0.117801 & -288.9618008 \\
\hline 4-trans & & 0.17809 & 0.145997 & -328.2808388 \\
\hline 4-cis & & 0.177832 & 0.145466 & -328.2814864 \\
\hline & & & tion States of C-N cle & avage \\
\hline $\mathrm{N}$ & ture & $\begin{array}{l}\text { Zero-point } \\
\text { Correction }\end{array}$ & $\begin{array}{c}\text { Thermal Correction } \\
\text { to Gibbs }\end{array}$ & electronic energy \\
\hline 1-trans & & 0.088284 & 0.060566 & 0.093606 \\
\hline 1-cis & & 0.087956 & 0.060191 & -210.1195264 \\
\hline 2-trans & & 0.118123 & 0.089158 & -249.5949126 \\
\hline
\end{tabular}




\begin{tabular}{|c|c|c|c|c|}
\hline $2-c i s$ & & 0.117644 & 0.088484 & -249.5941692 \\
\hline 3-trans & & 0.148158 & 0.117672 & -288.954353 \\
\hline 3-cis & & 0.147689 & 0.117185 & -288.9558114 \\
\hline 4-trans & & 0.177447 & 0.145602 & -328.2771554 \\
\hline 4-cis & & 0.176901 & 0.144763 & -328.277547 \\
\hline & & \multicolumn{3}{|c|}{ Transition States of C-C cleavage } \\
\hline $\mathrm{N}$ & Structure & $\begin{array}{l}\text { Zero-point } \\
\text { Correction }\end{array}$ & $\begin{array}{c}\text { Thermal Correction } \\
\text { to Gibbs }\end{array}$ & electronic energy \\
\hline 1-trans & & 0.087927 & 0.060184 & -210.099409 \\
\hline $1-c i s$ & & 0.087505 & 0.059715 & -210.1000864 \\
\hline 2-trans & & 0.117549 & 0.088455 & 0.123634 \\
\hline $2-c i s$ & & 0.117163 & 0.087947 & -249.5827455 \\
\hline 3-trans & & 0.147636 & 0.117064 & -288.9438959 \\
\hline 3-cis & & 0.147345 & 0.116815 & -288.9471299 \\
\hline 4-trans & & 0.176586 & 0.144349 & -328.2677428 \\
\hline 4-cis & & 0.176671 & 0.14476 & -328.2739177 \\
\hline
\end{tabular}


Table S7. The zero-point correction, thermal correction to Gibbs and electronic energy of the bicyclic 2-aziridinylmethyl radicals. (Unit: Hartree)

\begin{tabular}{|c|c|c|c|c|}
\hline \multirow[b]{2}{*}{$\mathrm{N}$} & \multirow[b]{2}{*}{ Structure } & \multicolumn{3}{|c|}{ Reactants } \\
\hline & & $\begin{array}{l}\text { Zero-point } \\
\text { Correction }\end{array}$ & $\begin{array}{c}\text { Thermal Correction } \\
\text { to Gibbs }\end{array}$ & electronic energy \\
\hline 3 & & 0.148213 & 0.117462 & -288.9614122 \\
\hline \multirow[t]{2}{*}{4} & & 0.177709 & 0.145643 & -328.28044 \\
\hline & & \multicolumn{3}{|c|}{ Transition States of C-N cleavage } \\
\hline $\mathrm{N}$ & $\mathrm{e}$ & $\begin{array}{l}\text { Zero-point } \\
\text { Correction } \\
\end{array}$ & $\begin{array}{c}\text { Thermal Correction } \\
\text { to Gibbs }\end{array}$ & electronic energy \\
\hline 3 & & 0.147998 & 0.117762 & -288.9574906 \\
\hline \multirow[t]{2}{*}{4} & & 0.177065 & 0.145416 & -328.2789327 \\
\hline & & \multicolumn{3}{|c|}{ Transition States of C-C cleavage } \\
\hline $\mathrm{N}$ & Structure & $\begin{array}{l}\text { Zero-point } \\
\text { Correction } \\
\end{array}$ & $\begin{array}{c}\text { Thermal Correction } \\
\text { to Gibbs }\end{array}$ & electronic energy \\
\hline 3 & & 0.14672 & 0.116326 & -288.9435966 \\
\hline 4 & & 0.176051 & 0.144252 & 0.184474 \\
\hline
\end{tabular}


Table S8. The zero-point correction, thermal correction to Gibbs and electronic energy of the bicyclic 2-aziridinylmethyl radicals. (Unit: Hartree)

\begin{tabular}{|c|c|c|c|c|}
\hline \multirow[b]{2}{*}{$\mathrm{N}$} & \multirow[b]{2}{*}{ Structure } & \multicolumn{3}{|c|}{ Reactants } \\
\hline & & $\begin{array}{l}\text { Zero-point } \\
\text { Correction }\end{array}$ & $\begin{array}{c}\text { Thermal Correction } \\
\text { to Gibbs } \\
\end{array}$ & electronic energy \\
\hline 2 & & 0.118515 & 0.089325 & 0.125237 \\
\hline 3 & & 0.148679 & 0.118126 & -288.9725921 \\
\hline \multirow[t]{2}{*}{4} & & 0.178118 & 0.14577 & -328.2958089 \\
\hline & & \multicolumn{3}{|c|}{ Transition States of C-N cleavage } \\
\hline $\mathrm{N}$ & re & $\begin{array}{l}\text { Zero-point } \\
\text { Correction }\end{array}$ & $\begin{array}{c}\text { Thermal Correction } \\
\text { to Gibbs } \\
\end{array}$ & electronic energy \\
\hline 2 & & 0.117696 & 0.08867 & -249.6089963 \\
\hline 3 & & 0.147791 & 0.117514 & -288.9587148 \\
\hline \multirow[t]{2}{*}{4} & & 0.177271 & 0.14563 & -328.2861523 \\
\hline & & \multicolumn{3}{|c|}{ Transition States of C-C cleavage } \\
\hline $\mathrm{N}$ & Str & $\begin{array}{l}\text { Zero-point } \\
\text { Correction }\end{array}$ & $\begin{array}{c}\text { Thermal Correction } \\
\text { to Gibbs }\end{array}$ & electronic energy \\
\hline 2 & & 0.117504 & 0.088389 & -249.5983472 \\
\hline 3 & & 0.148207 & 0.117985 & -288.9668608 \\
\hline 4 & & 0.177598 & 0.145802 & -328.292976 \\
\hline
\end{tabular}


Table S9. Spin values on C1 of all the radicals in Table S1-S3

\begin{tabular}{|c|c|c|c|}
\hline $\mathrm{R}_{2}{ }^{\mathrm{b}}$ & $\mathrm{R}_{1}$ & Reactants & $\begin{array}{c}\text { Transition States of } \\
\text { C-C cleavage }\end{array}$ \\
\hline $\mathrm{H}$ & cis-H & 0.080 & 0.697 \\
\hline $\mathrm{H}$ & trans $-\mathrm{CH}_{3}$ & 0.119 & 0.678 \\
\hline $\mathrm{H}$ & trans $-\mathrm{CH}_{2} \mathrm{CH}_{3}$ & 0.118 & 0.683 \\
\hline $\mathrm{H}$ & cis-CHO & 0.099 & 0.642 \\
\hline $\mathrm{H}$ & trans- $\mathrm{COCH}_{3}$ & 0.107 & 0.624 \\
\hline $\mathrm{H}$ & trans $-\mathrm{CONH}_{2}$ & 0.097 & 0.644 \\
\hline $\mathrm{H}$ & trans $-\mathrm{COOCH}_{3}$ & 0.106 & 0.637 \\
\hline $\mathrm{H}$ & trans $-\mathrm{Ph}$ & 0.116 & 0.650 \\
\hline cis- $\mathrm{COCH}_{3}$ & cis- $\mathrm{H}$ & 0.125 & 0.414 \\
\hline trans $-\mathrm{COCH}_{3}$ & trans $-\mathrm{H}$ & 0.135 & 0.409 \\
\hline$c i s-\mathrm{COOH}$ & $c i s-\mathrm{H}$ & 0.115 & 0.466 \\
\hline trans-COOH & trans $-\mathrm{H}$ & 0.127 & 0.461 \\
\hline cis- $\mathrm{CONH}_{2}$ & cis- $\mathrm{H}$ & 0.108 & 0.498 \\
\hline trans $-\mathrm{CONH}_{2}$ & cis- $\mathrm{H}$ & 0.098 & 0.521 \\
\hline cis $-\mathrm{CH}=\mathrm{CH}_{2}$ & cis- $\mathrm{H}$ & 0.114 & 0.417 \\
\hline trans $-\mathrm{CH}=\mathrm{CH}_{2}$ & cis- $\mathrm{H}$ & 0.109 & 0.387 \\
\hline cis $-\mathrm{C} \equiv \mathrm{CH}$ & cis- $\mathrm{H}$ & 0.090 & 0.445 \\
\hline trans $-\mathrm{C} \equiv \mathrm{CH}$ & trans $-\mathrm{H}$ & 0.129 & 0.416 \\
\hline$c i s-\mathrm{F}$ & cis- $\mathrm{H}$ & 0.058 & 0.606 \\
\hline trans $-\mathrm{F}$ & cis- $\mathrm{H}$ & 0.073 & 0.596 \\
\hline$c i s-\mathrm{NH}_{2}$ & cis- $\mathrm{H}$ & 0.075 & 0.549 \\
\hline trans $-\mathrm{NH}_{2}$ & cis- $\mathrm{H}$ & 0.078 & 0.464 \\
\hline cis- $-\mathrm{OCH}_{3}$ & $c i s-\mathrm{H}$ & 0.072 & 0.550 \\
\hline trans $-\mathrm{OCH}_{3}$ & cis- $\mathrm{H}$ & 0.080 & 0.523 \\
\hline cis $-\mathrm{CH}_{3}$ & cis- $\mathrm{H}$ & 0.091 & 0.625 \\
\hline trans $-\mathrm{CH}_{3}$ & cis- $\mathrm{H}$ & 0.082 & 0.653 \\
\hline cis- $-\mathrm{CH}_{3}$ & trans $-\mathrm{CH}_{3}$ & 0.131 & 0.634 \\
\hline trans $-\mathrm{CH}_{3}$ & cis $-\mathrm{CH}_{3}$ & 0.103 & 0.637 \\
\hline cis- $-\mathrm{CH}_{3}$ & trans $-\mathrm{CHO}$ & 0.121 & 0.577 \\
\hline trans $-\mathrm{CH}_{3}$ & cis-CHO & 0.104 & 0.590 \\
\hline cis- $-\mathrm{CH}_{3}$ & trans- $\mathrm{Ph}$ & 0.128 & 0.613 \\
\hline trans $-\mathrm{CH}_{3}$ & $c i s-\mathrm{Ph}$ & 0.100 & 0.640 \\
\hline cis-CHO & cis- $\mathrm{H}$ & 0.135 & 0.372 \\
\hline trans $-\mathrm{CHO}$ & trans $-\mathrm{H}$ & 0.142 & 0.367 \\
\hline cis-CHO & trans $-\mathrm{CH}_{3}$ & 0.155 & 0.414 \\
\hline trans-CHO & cis $-\mathrm{CH}_{3}$ & 0.141 & 0.386 \\
\hline cis-CHO & trans $-\mathrm{CHO}$ & 0.145 & 0.394 \\
\hline trans-CHO & cis-CHO & 0.132 & 0.377 \\
\hline cis-CHO & trans $-\mathrm{Ph}$ & 0.153 & 0.406 \\
\hline
\end{tabular}




$\begin{array}{cccc}\text { trans }-\mathrm{CHO} & \text { cis }-\mathrm{Ph} & 0.131 & 0.395 \\ \text { cis }-\mathrm{Ph} & \text { trans }-\mathrm{H} & 0.131 & 0.437 \\ \text { trans }-\mathrm{Ph} & \text { cis }-\mathrm{H} & 0.112 & 0.422 \\ \text { cis }-\mathrm{Ph} & \text { trans }-\mathrm{CH}_{3} & 0.142 & 0.424 \\ \text { trans }-\mathrm{Ph} & \text { cis }-\mathrm{CH}_{3} & 0.140 & 0.407 \\ \text { cis }-\mathrm{Ph} & \text { trans }-\mathrm{CHO} & 0.131 & 0.392 \\ \text { trans }-\mathrm{Ph} & \text { cis }-\mathrm{CHO} & 0.134 & 0.381 \\ \text { cis }-\mathrm{Ph} & \text { trans }-\mathrm{Ph} & 0.141 & 0.417 \\ \text { trans }-\mathrm{Ph} & \text { cis }-\mathrm{Ph} & 0.128 & 0.411\end{array}$


Table S10. Spin values on N of all the radicals in Table S1-S3

\begin{tabular}{|c|c|c|c|}
\hline $\mathrm{R}_{2}{ }^{\mathrm{b}}$ & $\mathrm{R}_{1}$ & Reactants & $\begin{array}{c}\text { Transition States of } \\
\text { C-N cleavage }\end{array}$ \\
\hline $\mathrm{H}$ & cis- $\mathrm{H}$ & 0.134 & 0.539 \\
\hline $\mathrm{H}$ & $\operatorname{trans}-\mathrm{CH}_{3}$ & 0.049 & 0.447 \\
\hline $\mathrm{H}$ & trans $-\mathrm{CH}_{2} \mathrm{CH}_{3}$ & 0.050 & 0.443 \\
\hline $\mathrm{H}$ & cis-CHO & 0.081 & 0.345 \\
\hline $\mathrm{H}$ & trans $-\mathrm{COCH}_{3}$ & 0.046 & 0.310 \\
\hline $\mathrm{H}$ & trans $-\mathrm{CONH}_{2}$ & 0.062 & 0.332 \\
\hline $\mathrm{H}$ & trans $-\mathrm{COOCH}_{3}$ & 0.046 & 0.312 \\
\hline $\mathrm{H}$ & trans $-\mathrm{Ph}$ & 0.043 & 0.306 \\
\hline cis- $\mathrm{COCH}_{3}$ & cis- $\mathrm{H}$ & 0.090 & 0.572 \\
\hline trans- $\mathrm{COCH}_{3}$ & trans $-\mathrm{H}$ & 0.036 & 0.543 \\
\hline cis-COOH & $c i s-\mathrm{H}$ & 0.097 & 0.568 \\
\hline trans $-\mathrm{COOH}$ & trans $-\mathrm{H}$ & 0.038 & 0.538 \\
\hline$c i s-\mathrm{CONH}_{2}$ & cis- $\mathrm{H}$ & 0.105 & 0.566 \\
\hline trans $-\mathrm{CONH}_{2}$ & cis- $\mathrm{H}$ & 0.100 & 0.545 \\
\hline cis $-\mathrm{CH}=\mathrm{CH}_{2}$ & cis- $\mathrm{H}$ & 0.104 & 0.570 \\
\hline trans $-\mathrm{CH}=\mathrm{CH}_{2}$ & cis- $\mathrm{H}$ & 0.099 & 0.569 \\
\hline cis $-\mathrm{C} \equiv \mathrm{CH}$ & cis- $\mathrm{H}$ & 0.122 & 0.564 \\
\hline trans $-\mathrm{C} \equiv \mathrm{CH}$ & trans $-\mathrm{H}$ & 0.039 & 0.523 \\
\hline cis-F & cis- $\mathrm{H}$ & 0.152 & 0.448 \\
\hline trans $-\mathrm{F}$ & cis- $\mathrm{H}$ & 0.108 & 0.512 \\
\hline$c i s-\mathrm{NH}_{2}$ & cis- $\mathrm{H}$ & 0.132 & 0.517 \\
\hline trans $-\mathrm{NH}_{2}$ & cis- $\mathrm{H}$ & 0.111 & 0.521 \\
\hline cis- $-\mathrm{OCH}_{3}$ & cis- $\mathrm{H}$ & 0.133 & 0.488 \\
\hline trans $-\mathrm{OCH}_{3}$ & cis- $\mathrm{H}$ & 0.112 & 0.506 \\
\hline cis- $\mathrm{CH}_{3}$ & $c i s-\mathrm{H}$ & 0.135 & 0.540 \\
\hline trans $-\mathrm{CH}_{3}$ & cis- $\mathrm{H}$ & 0.132 & 0.543 \\
\hline cis- $-\mathrm{CH}_{3}$ & trans $-\mathrm{CH}_{3}$ & 0.044 & 0.454 \\
\hline trans $-\mathrm{CH}_{3}$ & cis $-\mathrm{CH}_{3}$ & 0.114 & 0.462 \\
\hline cis $-\mathrm{CH}_{3}$ & trans $-\mathrm{CHO}$ & 0.038 & 0.305 \\
\hline trans $-\mathrm{CH}_{3}$ & cis-CHO & 0.077 & 0.353 \\
\hline cis $-\mathrm{CH}_{3}$ & trans-Ph & 0.039 & 0.313 \\
\hline trans $-\mathrm{CH}_{3}$ & $c i s-\mathrm{Ph}$ & 0.090 & 0.338 \\
\hline cis-CHO & cis- $\mathrm{H}$ & 0.079 & 0.573 \\
\hline trans-CHO & trans $-\mathrm{H}$ & 0.032 & 0.545 \\
\hline cis-CHO & trans $-\mathrm{CH}_{3}$ & 0.033 & 0.485 \\
\hline trans-CHO & cis $-\mathrm{CH}_{3}$ & 0.069 & 0.502 \\
\hline cis-CHO & trans $-\mathrm{CHO}$ & 0.028 & 0.317 \\
\hline trans-CHO & $c i s-\mathrm{CHO}$ & 0.056 & 0.356 \\
\hline cis-CHO & trans $-\mathrm{Ph}$ & 0.029 & 0.329 \\
\hline
\end{tabular}




$\begin{array}{cccc}\text { trans }-\mathrm{CHO} & \text { cis }-\mathrm{Ph} & 0.058 & 0.368 \\ \text { cis }-\mathrm{Ph} & \text { trans }-\mathrm{H} & 0.051 & 0.517 \\ \text { trans }-\mathrm{Ph} & \text { cis }-\mathrm{H} & 0.095 & 0.562 \\ \text { cis }-\mathrm{Ph} & \text { trans }-\mathrm{CH}_{3} & 0.046 & 0.472 \\ \text { trans }-\mathrm{Ph} & \text { cis }-\mathrm{CH}_{3} & 0.076 & 0.485 \\ \text { cis }-\mathrm{Ph} & \text { trans }-\mathrm{CHO} & 0.042 & 0.303 \\ \text { trans }-\mathrm{Ph} & \text { cis }-\mathrm{CHO} & 0.056 & 0.351 \\ \text { cis }-\mathrm{Ph} & \text { trans }-\mathrm{Ph} & 0.040 & 0.332 \\ \text { trans }-\mathrm{Ph} & \text { cis }-\mathrm{Ph} & 0.062 & 0.359\end{array}$


Table S11. The geometry of all radicals in Table S1-S8 2-aziridinylmethyl radical

\begin{tabular}{ccccc} 
Center & Atomic & \multicolumn{3}{c}{ Coordinates (Angstroms) } \\
Number & & $\mathrm{X}$ & $\mathrm{Y}$ & $\mathrm{Z}$ \\
$-\mathrm{-}$ & $\mathrm{C}$ & -0.205754 & -0.338978 & -0.076243 \\
1 & $\mathrm{C}$ & 1.225285 & -0.474587 & 0.114115 \\
2 & $\mathrm{~N}$ & 0.652354 & 0.807693 & -0.225898 \\
3 & $\mathrm{H}$ & 0.618820 & 1.364331 & 0.613800 \\
4 & $\mathrm{H}$ & 1.451741 & -0.471004 & 1.179304 \\
5 & $\mathrm{H}$ & 1.673154 & -0.893188 & -0.785944 \\
6 & $\mathrm{H}$ & -0.854699 & -1.206147 & 0.036933 \\
7 & $\mathrm{C}$ & -1.594705 & 0.077328 & -0.078694 \\
8 & $\mathrm{H}$ & -1.768893 & 0.765367 & 0.747249 \\
9 & $\mathrm{H}$ & -2.235557 & -0.795788 & 0.034876 \\
10 & & & &
\end{tabular}

trans- $N$-methyl 2-aziridinylmethyl radical

\begin{tabular}{ccccc} 
Center & Atomic & \multicolumn{3}{c}{ Coordinates (Angstroms) } \\
Number & & $\mathrm{X}$ & $\mathrm{Y}$ & $\mathrm{Z}$ \\
$-\mathrm{-}$ & & -1.942344 & -0.496857 & -0.120756 \\
1 & $\mathrm{C}$ & -0.649411 & -0.090694 & 0.412283 \\
2 & $\mathrm{C}$ & 0.505550 & -0.045781 & -0.504703 \\
3 & $\mathrm{~N}$ & 0.041526 & 1.213690 & 0.039915 \\
4 & $\mathrm{C}$ & 1.764212 & -0.555197 & 0.023649 \\
5 & $\mathrm{C}$ & -0.435416 & 1.893100 & -0.663410 \\
6 & $\mathrm{H}$ & -631047 & 1.695777 & 0.823265 \\
7 & $\mathrm{H}$ & -2.141256 & -0.412926 & -1.183323 \\
8 & $\mathrm{H}$ & -2.749085 & -0.786109 & 0.543627 \\
9 & $\mathrm{H}$ & 2.593809 & -0.111878 & -0.538811 \\
10 & $\mathrm{H}$ & 1.916594 & -0.331617 & 1.095303 \\
11 & $\mathrm{H}$ & 1.797862 & -1.641629 & -0.110112 \\
12 & $\mathrm{H}$ & & &
\end{tabular}

trans- $N$-ethyl 2-aziridinylmethyl radical

\begin{tabular}{llll} 
Center & Atomic & \multicolumn{3}{c}{ Coordinates (Angstroms) } \\
Number & & Y & Y
\end{tabular}




$\begin{array}{rrrrr}1 & \mathrm{~N} & 0.050953 & 0.358382 & -0.487994 \\ 2 & \mathrm{C} & -0.929257 & 1.311559 & -0.009626 \\ 3 & \mathrm{C} & -0.997292 & -0.147710 & 0.418592 \\ 4 & \mathrm{C} & -1.969756 & -1.095808 & -0.106953 \\ 5 & \mathrm{H} & -2.590882 & -1.680426 & 0.562619 \\ 6 & \mathrm{H} & -2.153466 & -1.148460 & -1.174258 \\ 7 & \mathrm{H} & -0.697652 & -0.302133 & 1.458468 \\ 8 & \mathrm{C} & 1.389574 & 0.467527 & 0.076870 \\ 9 & \mathrm{H} & -1.632268 & 1.688096 & -0.749716 \\ 10 & \mathrm{H} & -0.630736 & 2.032256 & 0.755268 \\ 11 & \mathrm{C} & 2.058562 & -0.814483 & -0.029920 \\ 12 & \mathrm{H} & 1.961436 & 1.204727 & -0.498138 \\ 13 & \mathrm{H} & 1.397784 & 0.777044 & 1.137899 \\ 14 & \mathrm{H} & 3.059886 & -0.737612 & 0.391224 \\ 15 & \mathrm{H} & 1.491553 & -1.566474 & 0.516827 \\ 16 & \mathrm{H} & 2.126681 & -1.102198 & -1.078014 \\ -------------------------------------------- & \end{array}$

cis- $N$-formyl 2-aziridinylmethyl radical

\begin{tabular}{ccccc} 
Center & Atomic & \multicolumn{3}{c}{ Coordinates (Angstroms) } \\
Number & & $\mathrm{X}$ & $\mathrm{Y}$ & $\mathrm{Z}$ \\
----1.635075 & -1.026902 & -0.201426 \\
1 & $\mathrm{C}$ & -1.231623 & 0.324282 & 0.160339 \\
2 & $\mathrm{C}$ & 0.104575 & 0.525391 & 0.758920 \\
3 & $\mathrm{~N}$ & -0.106075 & 1.083837 & -0.548587 \\
4 & $\mathrm{C}$ & -2.681548 & -1.268987 & -0.346048 \\
5 & $\mathrm{H}$ & -0.885349 & -1.781871 & -0.410099 \\
6 & $\mathrm{H}$ & -2.020169 & 0.973152 & 0.543735 \\
7 & $\mathrm{H}$ & 0.191572 & 2.167009 & -0.624686 \\
8 & $\mathrm{H}$ & 1.173281 & -0.336314 & 0.533472 \\
9 & $\mathrm{H}$ & 1.492067 & -0.846953 & 1.461128 \\
10 & $\mathrm{C}$ & 1.741802 & -0.472800 & -0.529206 \\
11 & $\mathrm{H}$ & & &
\end{tabular}

trans- $N$-carboxyl 2-aziridinylmethyl radical

\begin{tabular}{|c|c|c|c|c|}
\hline \multirow{2}{*}{$\begin{array}{l}\text { Center } \\
\text { Number }\end{array}$} & \multirow[t]{2}{*}{ Atomic } & \multicolumn{3}{|c|}{ Coordinates (Angstroms) } \\
\hline & & $X$ & $\mathrm{Y}$ & $\mathrm{Z}$ \\
\hline 1 & $\mathrm{C}$ & 2.426154 & 0.742427 & -0.122712 \\
\hline 2 & $\mathrm{C}$ & 1.272203 & 0.052404 & 0.435510 \\
\hline 3 & $\mathrm{C}$ & 0.870530 & -1.371291 & 0.037900 \\
\hline
\end{tabular}




$\begin{array}{rrrrr}4 & \mathrm{~N} & 0.128463 & -0.242420 & -0.452806 \\ 5 & \mathrm{C} & -1.193566 & -0.006158 & -0.089606 \\ 6 & \mathrm{O} & -2.037239 & -0.865517 & 0.054789 \\ 7 & \mathrm{H} & 1.478002 & -1.896453 & -0.695194 \\ 8 & \mathrm{H} & 0.394621 & -1.995986 & 0.792638 \\ 9 & \mathrm{H} & 1.014097 & 0.305975 & 1.464544 \\ 10 & \mathrm{H} & 2.632103 & 0.687381 & -1.185804 \\ 11 & \mathrm{H} & 3.133345 & 1.246220 & 0.525902 \\ 12 & \mathrm{C} & -1.476954 & 1.411647 & 0.020039 \\ 13 & \mathrm{H} & -2.551633 & 1.573447 & -0.049296 \\ 14 & \mathrm{H} & -1.115340 & 1.781045 & 0.978528 \\ 15 & \mathrm{H} & -0.976724 & 1.945268 & -0.786769\end{array}$

trans- $N$-acylamino 2-aziridinylmethyl radical

\begin{tabular}{crrrr} 
Center & Atomic & \multicolumn{3}{c}{ Coordinates (Angstroms) } \\
Number & & $\mathrm{X}$ & $\mathrm{Y}$ & $\mathrm{Z}$ \\
$-\mathrm{-}$ & & 2.431330 & 0.744369 & -0.099753 \\
1 & $\mathrm{C}$ & 1.279646 & 0.038329 & 0.442902 \\
2 & $\mathrm{C}$ & 0.867443 & -1.367237 & -0.005220 \\
3 & $\mathrm{C}$ & 0.125938 & -0.217102 & -0.444724 \\
4 & $\mathrm{~N}$ & -1.191368 & 0.010687 & -0.059610 \\
5 & $\mathrm{C}$ & -2.037618 & -0.849951 & 0.059950 \\
6 & $\mathrm{O}$ & 1.465275 & -1.866371 & -0.763974 \\
7 & $\mathrm{H}$ & 1.032767 & 0.253162 & 1.483444 \\
8 & $\mathrm{H}$ & 2.626656 & 0.729449 & -1.166165 \\
9 & $\mathrm{H}$ & 3.147161 & 1.219735 & 0.560732 \\
10 & $\mathrm{H}$ & -1.465118 & 1.414671 & 0.106187 \\
11 & $\mathrm{H}$ & -0.880709 & 1.947087 & -0.519164 \\
12 & $\mathrm{~N}$ & -2.434247 & 1.595176 & -0.104218 \\
13 & $\mathrm{H}$ & &
\end{tabular}

trans- $N$-acetoxyl 2-aziridinylmethyl radical

\begin{tabular}{|c|c|c|c|c|}
\hline \multirow{2}{*}{$\begin{array}{l}\text { Center } \\
\text { Number }\end{array}$} & \multirow[t]{2}{*}{ Atomic } & \multicolumn{3}{|c|}{ Coordinates (Angstroms) } \\
\hline & & $\mathrm{X}$ & $\mathrm{Y}$ & $\mathrm{Z}$ \\
\hline 1 & $\mathrm{C}$ & 1.849206 & 1.581021 & 0.265013 \\
\hline 2 & $\mathrm{C}$ & 1.712217 & 0.137507 & 0.266379 \\
\hline 3 & $\mathrm{~N}$ & 0.782577 & -0.269958 & -0.755062 \\
\hline 4 & $\mathrm{C}$ & 1.574349 & -1.266100 & -0.070301 \\
\hline 5 & $\mathrm{C}$ & -0.547932 & -0.308080 & -0.205615 \\
\hline
\end{tabular}




$\begin{array}{rrrrc}6 & \text { H } & 2.387926 & -1.562605 & -0.730679 \\ 7 & \text { H } & 0.937980 & -1.755148 & 0.665761 \\ 8 & \text { H } & 2.415256 & 0.445657 & 1.038844 \\ 9 & \text { H } & 2.216927 & 1.909837 & -0.705854 \\ 10 & \text { H } & 2.553555 & 1.879543 & 1.040062 \\ 11 & \text { O } & -1.430612 & 0.754161 & -0.434789 \\ 12 & \text { O } & -0.905977 & -1.266045 & 0.459597 \\ 13 & \text { C } & -2.652608 & 0.489682 & 0.195112 \\ 14 & \text { H } & -3.339207 & 1.315954 & 0.016848 \\ 15 & \text { H } & -3.077370 & -0.428604 & -0.207690 \\ 16 & \text { H } & -2.491786 & 0.375968 & 1.266152\end{array}$

trans- $N$-phenyl 2-aziridinylmethyl radical

\begin{tabular}{|c|c|c|c|c|}
\hline \multirow{2}{*}{$\begin{array}{l}\text { Center } \\
\text { Number }\end{array}$} & \multirow[t]{2}{*}{ Atomic } & \multicolumn{3}{|c|}{ Coordinates (Angstroms) } \\
\hline & & $\mathrm{X}$ & $\mathrm{Y}$ & $\mathrm{Z}$ \\
\hline 1 & $\mathrm{C}$ & 2.530725 & 0.920876 & -1.031735 \\
\hline 2 & $\mathrm{C}$ & 2.159884 & 0.178694 & 0.157442 \\
\hline 3 & $\mathrm{~N}$ & 1.251025 & -0.883393 & -0.188314 \\
\hline 4 & $\mathrm{C}$ & 1.818698 & -0.832020 & 1.139545 \\
\hline 5 & $\mathrm{C}$ & -0.103420 & -0.400852 & -0.109355 \\
\hline 6 & $\mathrm{H}$ & 2.617662 & -1.570033 & 1.193534 \\
\hline 7 & $\mathrm{H}$ & 1.060332 & -0.439883 & 1.815585 \\
\hline 8 & $\mathrm{H}$ & 2.847209 & 0.981898 & 0.418917 \\
\hline 9 & $\mathrm{H}$ & 3.015218 & 0.251490 & -1.741038 \\
\hline 10 & $\mathrm{H}$ & 3.217581 & 1.721280 & -0.760616 \\
\hline 11 & $\mathrm{C}$ & -1.170289 & -1.258416 & -0.403252 \\
\hline 12 & $\mathrm{C}$ & -2.487110 & -0.789280 & -0.326487 \\
\hline 13 & $\mathrm{C}$ & -2.737062 & 0.537420 & 0.044176 \\
\hline 14 & $\mathrm{C}$ & -1.670194 & 1.394984 & 0.338073 \\
\hline 15 & $\mathrm{C}$ & -0.353372 & 0.925848 & 0.261307 \\
\hline 16 & $\mathrm{H}$ & -0.975861 & -2.290399 & -0.691575 \\
\hline 17 & $\mathrm{H}$ & -3.316981 & -1.456342 & -0.555097 \\
\hline 18 & $\mathrm{H}$ & -3.761361 & 0.902341 & 0.103888 \\
\hline 19 & $\mathrm{H}$ & -1.864621 & 2.426967 & 0.626395 \\
\hline 20 & $\mathrm{H}$ & 0.476499 & 1.592910 & 0.489917 \\
\hline
\end{tabular}

transition state of $\mathrm{C}-\mathrm{N}$ cleavage of 2-aziridinylmethyl radical

\begin{tabular}{llcr} 
Center & Atomic & \multicolumn{3}{c}{ Coordinates (Angstroms) } \\
Number & $\mathrm{X}$ & $\mathrm{Y}$
\end{tabular}




$\begin{array}{rrrrc}1 & \text { N } & 1.011187 & 0.726736 & -0.298354 \\ 2 & \mathrm{C} & -0.363489 & -0.433190 & -0.367891 \\ 3 & \mathrm{C} & 0.919852 & -0.535390 & 0.401908 \\ 4 & \mathrm{H} & 0.678243 & -0.317479 & 1.441168 \\ 5 & \mathrm{H} & 1.545640 & -1.251346 & -0.128863 \\ 6 & \mathrm{H} & 0.786736 & 1.476040 & 0.337435 \\ 7 & \mathrm{H} & -0.357263 & -1.239983 & -1.099305 \\ 8 & \mathrm{C} & -1.572050 & 0.220306 & 0.095600 \\ 9 & \mathrm{H} & -1.358501 & 1.265513 & 0.314371 \\ 10 & \mathrm{H} & -2.279042 & -0.530246 & 0.445965\end{array}$

transition state of C-N cleavage of trans- $N$-methyl 2-aziridinylmethyl radical

\begin{tabular}{ccccc} 
Center & Atomic & \multicolumn{3}{c}{ Coordinates (Angstroms) } \\
Number & & $\mathrm{X}$ & $\mathrm{Y}$ & $\mathrm{Z}$ \\
\hline 1 & $\mathrm{~N}$ & 0.608376 & -0.157528 & -0.605664 \\
2 & $\mathrm{C}$ & -0.793583 & 0.052785 & 0.503515 \\
3 & $\mathrm{C}$ & 0.062968 & 1.099991 & -0.144312 \\
4 & $\mathrm{H}$ & -0.523397 & 1.522159 & -0.959094 \\
5 & $\mathrm{H}$ & 0.727353 & 1.481833 & 0.629452 \\
6 & $\mathrm{C}$ & 1.784637 & -0.474365 & 0.162206 \\
7 & $\mathrm{H}$ & -0.629913 & 0.131849 & 1.577238 \\
8 & $\mathrm{C}$ & -1.972151 & -0.552090 & -0.086064 \\
9 & $\mathrm{H}$ & -1.702133 & -1.033247 & -1.024946 \\
10 & $\mathrm{H}$ & -2.865231 & -0.095117 & 0.337617 \\
11 & $\mathrm{H}$ & 2.632823 & -0.601604 & -0.508848 \\
12 & $\mathrm{H}$ & 1.991945 & 0.335856 & 0.859692 \\
13 & $\mathrm{H}$ & 1.618692 & -1.396953 & 0.716474
\end{tabular}

transition state of C-N cleavage of trans- $N$-ethyl 2-aziridinylmethyl radical

\begin{tabular}{ccccc} 
Center & Atomic & \multicolumn{3}{c}{ Coordinates (Angstroms) } \\
Number & & $\mathrm{X}$ & $\mathrm{Y}$ & $\mathrm{Z}$ \\
$-\mathrm{-}$ & $\mathrm{N}$ & 0.161038 & 0.423949 & -0.580319 \\
1 & $\mathrm{C}$ & -0.863222 & 1.236141 & 0.027108 \\
2 & $\mathrm{C}$ & -1.165789 & -0.155310 & 0.466662 \\
3 & $\mathrm{C}$ & -2.118934 & -0.988628 & -0.120159 \\
4 & $\mathrm{H}$ & -2.287559 & -1.993437 & 0.252635 \\
5 & $\mathrm{H}$ & -2.654292 & -0.686540 & -1.014750 \\
6 & $\mathrm{H}$ & -0.705373 & -0.468948 & 1.399207 \\
7 & & & &
\end{tabular}




$\begin{array}{rrrrc}8 & \mathrm{C} & 1.427559 & 0.429439 & 0.140338 \\ 9 & \mathrm{H} & -1.563235 & 1.674680 & -0.687516 \\ 10 & \mathrm{H} & -0.568560 & 1.946627 & 0.808100 \\ 11 & \mathrm{H} & 1.345058 & 0.615953 & 1.224842 \\ 12 & \mathrm{C} & 2.117080 & -0.829770 & -0.063259 \\ 13 & \mathrm{H} & 2.057433 & 1.227818 & -0.281740 \\ 14 & \mathrm{H} & 3.061946 & -0.817900 & 0.478044 \\ 15 & \mathrm{H} & 1.497918 & -1.646377 & 0.305132 \\ 16 & \mathrm{H} & 2.309231 & -0.970744 & -1.125862\end{array}$

transition state of C-N cleavage of cis- $N$-formyl 2-aziridinylmethyl radical

\begin{tabular}{|c|c|c|c|c|}
\hline \multirow{2}{*}{$\begin{array}{l}\text { Center } \\
\text { Number }\end{array}$} & \multirow[t]{2}{*}{ Atomic } & \multicolumn{3}{|c|}{ Coordinates (Angstroms) } \\
\hline & & $\mathrm{X}$ & $\mathrm{Y}$ & $\mathrm{Z}$ \\
\hline 1 & $\mathrm{~N}$ & 0.268817 & -0.634368 & -0.524477 \\
\hline 2 & $\mathrm{C}$ & -1.449950 & -0.164626 & -0.269166 \\
\hline 3 & $\mathrm{C}$ & -0.619264 & -1.155602 & 0.491051 \\
\hline 4 & $\mathrm{H}$ & -0.353559 & -0.681048 & 1.434513 \\
\hline 5 & $\mathrm{H}$ & -0.941559 & -2.144725 & 0.169053 \\
\hline 6 & $\mathrm{C}$ & 1.130211 & 0.358677 & 0.063283 \\
\hline 7 & $\mathrm{H}$ & -2.245148 & -0.727538 & -0.755697 \\
\hline 8 & $\mathrm{C}$ & -1.527137 & 1.256386 & 0.008804 \\
\hline 9 & $\mathrm{H}$ & -0.533062 & 1.695046 & -0.064090 \\
\hline 10 & $\mathrm{H}$ & -2.445549 & 1.470361 & 0.553464 \\
\hline 11 & $\mathrm{O}$ & 2.341722 & 0.229288 & 0.000844 \\
\hline 12 & $\mathrm{H}$ & 0.700218 & 1.225164 & 0.563511 \\
\hline
\end{tabular}

transition state of C-N cleavage of trans- $\mathrm{N}$-carboxyl 2-aziridinylmethyl radical

\begin{tabular}{ccccc} 
Center & Atomic & \multicolumn{3}{c}{ Coordinates (Angstroms) } \\
Number & & $\mathrm{X}$ & $\mathrm{Y}$ & $\mathrm{Z}$ \\
$-\mathrm{-}$ & & 2.514390 & 0.703784 & -0.022135 \\
1 & $\mathrm{C}$ & 1.421570 & 0.021432 & 0.505309 \\
2 & $\mathrm{C}$ & 0.863943 & -1.252480 & -0.048289 \\
3 & $\mathrm{C}$ & 0.049404 & -0.224316 & -0.631619 \\
4 & $\mathrm{~N}$ & 2.867293 & 1.623961 & 0.430462 \\
5 & $\mathrm{H}$ & 2.994257 & 0.371215 & -0.937101 \\
6 & $\mathrm{H}$ & 0.988250 & 0.365551 & 1.439421 \\
7 & $\mathrm{H}$ & 0.394468 & -1.929156 & 0.675311 \\
8 & $\mathrm{H}$ & 1.497127 & -1.782608 & -0.762413 \\
9 & $\mathrm{H}$ & -1.222256 & -0.025376 & -0.093921 \\
10 & $\mathrm{C}$ & & &
\end{tabular}




$\begin{array}{llrrr}11 & \mathrm{O} & -1.994168 & -0.899869 & 0.235818 \\ 12 & \mathrm{C} & -1.591392 & 1.375955 & -0.043813 \\ 13 & \mathrm{H} & -2.673897 & 1.463543 & 0.036385 \\ 14 & \mathrm{H} & -1.124255 & 1.843670 & 0.821604 \\ 15 & \mathrm{H} & -1.253257 & 1.873100 & -0.951796\end{array}$

transition state of C-N cleavage of trans- $\mathrm{N}$-acylamino 2-aziridinylmethyl radical

\begin{tabular}{ccrrc} 
Center & Atomic & \multicolumn{3}{c}{ Coordinates (Angstroms) } \\
Number & & X & Y & Z \\
- & & 2.515986 & 0.671645 & -0.029080 \\
1 & $\mathrm{C}$ & 1.405507 & 0.026131 & 0.507766 \\
2 & $\mathrm{C}$ & 0.835494 & -1.258911 & -0.006065 \\
3 & $\mathrm{C}$ & 0.045224 & -0.240947 & -0.638591 \\
4 & $\mathrm{~N}$ & 2.877131 & 1.602586 & 0.394045 \\
5 & $\mathrm{H}$ & 3.002955 & 0.297931 & -0.924198 \\
6 & $\mathrm{H}$ & 0.964996 & 0.411393 & 1.422247 \\
7 & $\mathrm{H}$ & 1.469911 & -1.824866 & -0.691003 \\
8 & $\mathrm{H}$ & -1.230340 & -0.002780 & -0.126705 \\
9 & $\mathrm{H}$ & -2.020316 & -0.852530 & 0.224261 \\
10 & $\mathrm{C}$ & -1.575647 & 1.395188 & -0.133770 \\
11 & $\mathrm{O}$ & -2.205440 & 1.589581 & 0.628881 \\
12 & $\mathrm{~N}$ & -0.739586 & 1.948335 & -0.028441 \\
13 & $\mathrm{H}$ & &
\end{tabular}

transition state of C-N cleavage of trans- $\mathrm{N}$-acetoxyl 2-aziridinylmethyl radical

\begin{tabular}{ccccc} 
Center & Atomic & \multicolumn{3}{c}{ Coordinates (Angstroms) } \\
Number & & $\mathrm{X}$ & $\mathrm{Y}$ & $\mathrm{Z}$ \\
$-\mathrm{-}$ & $\mathrm{N}$ & -0.626855 & 0.284930 & -0.741312 \\
1 & $\mathrm{C}$ & -1.797199 & -0.138797 & 0.558976 \\
2 & $\mathrm{C}$ & -1.720089 & 1.107430 & -0.272270 \\
3 & $\mathrm{H}$ & -2.519962 & 1.041259 & -1.008304 \\
4 & $\mathrm{H}$ & -1.297692 & 1.882214 & 0.365862 \\
5 & $\mathrm{C}$ & 0.708385 & 0.290561 & -0.202143 \\
6 & $\mathrm{H}$ & -1.596431 & 0.155889 & 1.587943 \\
7 & $\mathrm{C}$ & -2.507516 & -1.349396 & 0.195107 \\
8 & $\mathrm{H}$ & -2.114597 & -1.731934 & -0.745744 \\
9 & $\mathrm{H}$ & -3.457492 & -1.386424 & 0.726234 \\
10 & $\mathrm{O}$ & 1.548835 & -0.811039 & -0.402449 \\
11 & $\mathrm{O}$ & 1.107238 & 1.255297 & 0.429206 \\
12 & & & &
\end{tabular}




$\begin{array}{rrrrr}13 & \mathrm{C} & 2.784748 & -0.572644 & 0.210483 \\ 14 & \mathrm{H} & 3.438498 & -1.429531 & 0.054674 \\ 15 & \mathrm{H} & 3.240470 & 0.315510 & -0.224743 \\ 16 & \mathrm{H} & 2.636640 & -0.418474 & 1.278292\end{array}$

transition state of C-N cleavage of trans- $N$-phenyl 2-aziridinylmethyl radical

\begin{tabular}{ccccc} 
Center & Atomic & \multicolumn{3}{c}{ Coordinates (Angstroms) } \\
Number & & $\mathrm{X}$ & $\mathrm{Y}$ & $\mathrm{Z}$ \\
\hline 1 & $\mathrm{~N}$ & 1.133007 & -0.884050 & -0.089853 \\
2 & $\mathrm{C}$ & 2.260476 & 0.512268 & 0.048387 \\
3 & $\mathrm{C}$ & 1.802909 & -0.440529 & 1.112723 \\
4 & $\mathrm{H}$ & 2.631384 & -1.122703 & 1.297620 \\
5 & $\mathrm{H}$ & 1.072154 & 0.094774 & 1.717182 \\
6 & $\mathrm{C}$ & -0.225588 & -0.406862 & -0.079233 \\
7 & $\mathrm{H}$ & 1.877652 & 1.495037 & 0.319555 \\
8 & $\mathrm{C}$ & 3.394329 & 0.307774 & -0.831988 \\
9 & $\mathrm{H}$ & 3.252023 & -0.610491 & -1.399842 \\
10 & $\mathrm{H}$ & 4.241939 & 0.889938 & -0.473429 \\
11 & $\mathrm{C}$ & -1.287091 & -1.314522 & -0.175995 \\
12 & $\mathrm{C}$ & -2.607947 & -0.850590 & -0.165670 \\
13 & $\mathrm{C}$ & -2.867299 & 0.521004 & -0.058582 \\
14 & $\mathrm{C}$ & -1.805796 & 1.428664 & 0.038180 \\
15 & $\mathrm{C}$ & -0.484940 & 0.964732 & 0.027855 \\
16 & $\mathrm{H}$ & -1.085352 & -2.381426 & -0.259294 \\
17 & $\mathrm{H}$ & -3.433645 & -1.556620 & -0.240938 \\
18 & $\mathrm{H}$ & -3.894736 & 0.881877 & -0.050551 \\
19 & $\mathrm{H}$ & -2.007535 & 2.495568 & 0.121479 \\
20 & $\mathrm{H}$ & 0.340757 & 1.670762 & 0.103122
\end{tabular}

transition state of C-C cleavage of 2-aziridinylmethyl radical

\begin{tabular}{|c|c|c|c|c|}
\hline \multirow{2}{*}{$\begin{array}{l}\text { Center } \\
\text { Number }\end{array}$} & \multirow[t]{2}{*}{ Atomic } & \multicolumn{3}{|c|}{ Coordinates (Angstroms) } \\
\hline & & $\mathrm{X}$ & $\mathrm{Y}$ & $\mathrm{Z}$ \\
\hline 1 & $\mathrm{~N}$ & 0.624653 & 0.596954 & -0.468442 \\
\hline 2 & $\mathrm{C}$ & -0.343769 & -0.459131 & -0.325461 \\
\hline 3 & $\mathrm{C}$ & 1.276695 & -0.264700 & 0.433658 \\
\hline 4 & $\mathrm{H}$ & 1.159242 & 0.112335 & 1.448533 \\
\hline 5 & $\mathrm{H}$ & 2.004052 & -0.872360 & -0.102645 \\
\hline 6 & $\mathrm{H}$ & 0.358829 & 1.377385 & 0.111496 \\
\hline
\end{tabular}




$\begin{array}{rrrrr}7 & \mathrm{H} & -0.315966 & -1.317689 & -0.994809 \\ 8 & \mathrm{C} & -1.586440 & 0.074923 & 0.197086 \\ 9 & \mathrm{H} & -2.174640 & -0.732472 & 0.630739 \\ 10 & \mathrm{H} & -1.483000 & 1.147570 & 0.354086\end{array}$

transition state of C-C cleavage of trans- $N$-methyl 2-aziridinylmethyl radical

\begin{tabular}{crrrr} 
Center & Atomic & \multicolumn{3}{c}{ Coordinates (Angstroms) } \\
Number & Number & X & Y & Z \\
- & C & -1.962723 & -0.368966 & -0.145200 \\
1 & $\mathrm{C}$ & -0.686762 & -0.087649 & 0.483517 \\
2 & $\mathrm{C}$ & 0.430223 & 1.247620 & 0.025945 \\
3 & $\mathrm{C}$ & 0.354692 & -0.051655 & -0.510311 \\
4 & $\mathrm{~N}$ & -0.087382 & 1.944677 & -0.631413 \\
5 & $\mathrm{H}$ & 1.247369 & 1.299431 & 0.743934 \\
6 & $\mathrm{H}$ & -0.458081 & -0.402178 & 1.500718 \\
7 & $\mathrm{C}$ & -2.769782 & -0.052787 & 0.514047 \\
8 & $\mathrm{H}$ & -1.820787 & -0.484584 & -1.218703 \\
9 & $\mathrm{H}$ & 2.324010 & -0.662396 & -0.742139 \\
10 & $\mathrm{H}$ & 1.869306 & -0.164124 & 0.903287 \\
11 & $\mathrm{H}$ & 1.309600 & -1.724788 & 0.260235 \\
12 & $\mathrm{H}$ & &
\end{tabular}

transition state of C-C cleavage of trans- $N$-ethyl 2-aziridinylmethyl radical

\begin{tabular}{ccccc} 
Center & Atomic & \multicolumn{3}{c}{ Coordinates (Angstroms) } \\
Number & & $\mathrm{X}$ & $\mathrm{Y}$ & $\mathrm{Z}$ \\
\hline 1 & $\mathrm{~N}$ & -0.031820 & 0.405639 & -0.391647 \\
2 & $\mathrm{C}$ & -0.886268 & 1.496119 & -0.090529 \\
3 & $\mathrm{C}$ & -0.974659 & -0.315813 & 0.418297 \\
4 & $\mathrm{C}$ & -1.894474 & -1.191380 & -0.112173 \\
5 & $\mathrm{H}$ & -2.621688 & -1.681936 & 0.526163 \\
6 & $\mathrm{H}$ & -1.960654 & -1.343600 & -1.184222 \\
7 & $\mathrm{H}$ & -0.843248 & -0.248552 & 1.502844 \\
8 & $\mathrm{C}$ & 1.332708 & 0.486953 & 0.128549 \\
9 & $\mathrm{H}$ & -1.632584 & 1.781833 & -0.822179 \\
10 & $\mathrm{H}$ & -0.663675 & 2.161375 & 0.744956 \\
11 & $\mathrm{C}$ & 2.082974 & -0.825283 & -0.080967 \\
12 & $\mathrm{H}$ & 1.829981 & 1.305037 & -0.406164 \\
13 & $\mathrm{H}$ & 1.333396 & 0.757883 & 1.203090 \\
14 & $\mathrm{H}$ & 3.104858 & -0.749142 & 0.306347
\end{tabular}




$\begin{array}{lllll}15 & \mathrm{H} & 1.583867 & -1.651305 & 0.437398 \\ 16 & \mathrm{H} & 2.130804 & -1.074631 & -1.145757\end{array}$

transition state of C-C cleavage of $c i s-N$-formyl 2-aziridinylmethyl radical

\begin{tabular}{|c|c|c|c|c|}
\hline \multirow{2}{*}{$\begin{array}{l}\text { Center } \\
\text { Number }\end{array}$} & \multirow[t]{2}{*}{ Atomic } & \multicolumn{3}{|c|}{ Coordinates (Angstroms) } \\
\hline & & $\mathrm{X}$ & $\mathrm{Y}$ & $\mathrm{Z}$ \\
\hline 1 & $\mathrm{C}$ & -1.243577 & -1.227175 & -0.232516 \\
\hline 2 & $\mathrm{C}$ & -1.267925 & 0.196883 & 0.039447 \\
\hline 3 & $\mathrm{C}$ & 0.015410 & 1.370225 & -0.425623 \\
\hline 4 & $\mathrm{~N}$ & -0.070942 & 0.585135 & 0.739524 \\
\hline 5 & $\mathrm{H}$ & -1.937295 & -1.454313 & -1.040653 \\
\hline 6 & $\mathrm{H}$ & -0.322989 & -1.656104 & 0.160496 \\
\hline 7 & $\mathrm{H}$ & -2.196453 & 0.741216 & 0.205158 \\
\hline 8 & $\mathrm{H}$ & 0.697662 & 0.900627 & -1.132679 \\
\hline 9 & $\mathrm{H}$ & -0.322829 & 2.382603 & -0.209737 \\
\hline 10 & $\mathrm{C}$ & 0.947778 & -0.412529 & 0.538342 \\
\hline 11 & $\mathrm{H}$ & 1.119537 & -1.175815 & 1.295848 \\
\hline 12 & $\mathrm{O}$ & 1.593606 & -0.424823 & -0.496626 \\
\hline
\end{tabular}

transition state of C-C cleavage of trans- $\mathrm{N}$-carboxyl 2-aziridinylmethyl radical

\begin{tabular}{|c|c|c|c|c|}
\hline \multirow{2}{*}{$\begin{array}{l}\text { Center } \\
\text { Number }\end{array}$} & \multirow[t]{2}{*}{ Atomic } & \multicolumn{3}{|c|}{ Coordinates (Angstroms) } \\
\hline & & $\mathrm{X}$ & $\mathrm{Y}$ & $\mathrm{Z}$ \\
\hline 1 & $\mathrm{C}$ & -2.372092 & -0.717251 & -0.219168 \\
\hline 2 & $\mathrm{C}$ & -1.282880 & -0.159032 & 0.425793 \\
\hline 3 & $\mathrm{~N}$ & -0.147801 & 0.341357 & -0.288409 \\
\hline 4 & $\mathrm{C}$ & 1.166027 & -0.041496 & -0.079714 \\
\hline 5 & $\mathrm{O}$ & 2.108351 & 0.722420 & -0.059038 \\
\hline 6 & $\mathrm{C}$ & -0.693898 & 1.590080 & 0.060223 \\
\hline 7 & $\mathrm{H}$ & -1.311304 & 2.095714 & -0.673036 \\
\hline 8 & $\mathrm{H}$ & -0.259776 & 2.149921 & 0.885373 \\
\hline 9 & $\mathrm{H}$ & -1.182767 & -0.237558 & 1.507767 \\
\hline 10 & $\mathrm{H}$ & -3.228969 & -1.060682 & 0.349793 \\
\hline 11 & $\mathrm{H}$ & -2.423026 & -0.748424 & -1.302262 \\
\hline 12 & $\mathrm{C}$ & 1.286676 & -1.480131 & 0.055467 \\
\hline 13 & $\mathrm{H}$ & 2.222979 & -1.720349 & 0.557017 \\
\hline 14 & $\mathrm{H}$ & 0.452274 & -1.860800 & 0.642651 \\
\hline 15 & $\mathrm{H}$ & 1.275386 & -1.939708 & -0.931743 \\
\hline
\end{tabular}


transition state of C-C cleavage of trans- $\mathrm{N}$-acylamino 2-aziridinylmethyl radical

\begin{tabular}{ccccc} 
Center & Atomic & \multicolumn{3}{c}{ Coordinates (Angstroms) } \\
Number & & $\mathrm{X}$ & $\mathrm{Y}$ & $\mathrm{Z}$ \\
\hline 1 & $\mathrm{C}$ & -2.374621 & -0.711190 & -0.202004 \\
2 & $\mathrm{C}$ & -1.287721 & -0.139851 & 0.435326 \\
3 & $\mathrm{~N}$ & -0.140226 & 0.325073 & -0.282990 \\
4 & $\mathrm{C}$ & 1.167667 & -0.061451 & -0.045734 \\
5 & $\mathrm{O}$ & 2.116557 & 0.694562 & -0.041230 \\
6 & $\mathrm{C}$ & -0.678907 & 1.590043 & 0.014918 \\
7 & $\mathrm{H}$ & -1.283453 & 2.074646 & -0.742847 \\
8 & $\mathrm{H}$ & -0.249038 & 2.175045 & 0.824697 \\
9 & $\mathrm{H}$ & -1.200472 & -0.180797 & 1.520485 \\
10 & $\mathrm{H}$ & -3.240895 & -1.026814 & 0.368828 \\
11 & $\mathrm{H}$ & -2.413673 & -0.780334 & -1.283832 \\
12 & $\mathrm{~N}$ & 1.273066 & -1.485482 & 0.140354 \\
13 & $\mathrm{H}$ & 0.462900 & -1.936825 & -0.254594 \\
14 & $\mathrm{H}$ & 2.103793 & -1.823855 & -0.319493 \\
------------1 &
\end{tabular}

transition state of C-C cleavage of trans- $\mathrm{N}$-acetoxyl 2-aziridinylmethyl radical

\begin{tabular}{crrrr} 
Center & Atomic & \multicolumn{3}{c}{ Coordinates (Angstroms) } \\
Number & & $\mathrm{X}$ & $\mathrm{Y}$ & $\mathrm{Z}$ \\
-- & & -2.256741 & -1.479425 & 0.048768 \\
1 & $\mathrm{C}$ & -1.562142 & -0.307062 & 0.544345 \\
2 & $\mathrm{C}$ & -1.669456 & 1.345442 & -0.161124 \\
3 & $\mathrm{C}$ & -0.798665 & 0.298022 & -0.516123 \\
4 & $\mathrm{~N}$ & -2.498424 & 1.386012 & -0.866168 \\
5 & $\mathrm{H}$ & -1.148394 & 2.050469 & 0.484904 \\
6 & $\mathrm{H}$ & -1.221226 & -0.225980 & 1.575424 \\
7 & $\mathrm{C}$ & -3.082945 & -1.720873 & 0.715859 \\
8 & $\mathrm{H}$ & -2.030351 & -1.615953 & -1.007655 \\
9 & $\mathrm{H}$ & 1.368951 & -0.845364 & -0.307052 \\
10 & $\mathrm{H}$ & 1.102860 & 1.331996 & 0.280056 \\
11 & $\mathrm{O}$ & 2.681462 & -0.586558 & 0.105665 \\
12 & $\mathrm{O}$ & 3.283039 & -1.486706 & -0.011638 \\
13 & $\mathrm{C}$ & 3.102176 & 0.212995 & -0.502311 \\
14 & $\mathrm{H}$ & 2.680116 & -0.284650 & 1.151977 \\
15 & $\mathrm{H}$ & &
\end{tabular}

transition state of C-C cleavage of trans- $N$-phenyl 2-aziridinylmethyl radical 


\begin{tabular}{|c|c|c|c|c|}
\hline \multirow{2}{*}{$\begin{array}{l}\text { Center } \\
\text { Number }\end{array}$} & \multirow[t]{2}{*}{ Atomic } & \multicolumn{3}{|c|}{ Coordinates (Angstroms) } \\
\hline & & $X$ & Y & $\mathrm{Z}$ \\
\hline 1 & $\mathrm{C}$ & 3.244025 & 0.487657 & -0.809860 \\
\hline 2 & $\mathrm{C}$ & 2.087522 & 0.507628 & 0.064555 \\
\hline 3 & $\mathrm{C}$ & 1.609487 & -0.810796 & 1.192922 \\
\hline 4 & $\mathrm{~N}$ & 1.275512 & -0.659023 & -0.166055 \\
\hline 5 & $\mathrm{H}$ & 2.429774 & -1.520414 & 1.290352 \\
\hline 6 & $\mathrm{H}$ & 0.779407 & -0.474981 & 1.812693 \\
\hline 7 & $\mathrm{C}$ & -0.116122 & -0.291562 & -0.122177 \\
\hline 8 & $\mathrm{H}$ & 1.624891 & 1.436318 & 0.395355 \\
\hline 9 & $\mathrm{H}$ & 4.014180 & 1.144505 & -0.408197 \\
\hline 10 & $\mathrm{H}$ & 3.215489 & -0.407692 & -1.429098 \\
\hline 11 & $\mathrm{C}$ & -1.103505 & -1.264188 & -0.319853 \\
\hline 12 & $\mathrm{C}$ & -2.456483 & -0.906934 & -0.277195 \\
\hline 13 & $\mathrm{C}$ & -2.822079 & 0.422945 & -0.036860 \\
\hline 14 & $\mathrm{C}$ & -1.834696 & 1.395571 & 0.160816 \\
\hline 15 & $\mathrm{C}$ & -0.481718 & 1.038317 & 0.118157 \\
\hline 16 & $\mathrm{H}$ & -0.819123 & -2.298644 & -0.506799 \\
\hline 17 & $\mathrm{H}$ & -3.224525 & -1.663498 & -0.430958 \\
\hline 18 & $\mathrm{H}$ & -3.874503 & 0.700838 & -0.003677 \\
\hline 19 & $\mathrm{H}$ & -2.119078 & 2.430027 & 0.347762 \\
\hline 20 & $\mathrm{H}$ & 0.286324 & 1.794881 & 0.271921 \\
\hline
\end{tabular}

cis- $C_{1}$-methyl 2-aziridinylmethyl radical

\begin{tabular}{ccccc} 
Center & Atomic & \multicolumn{3}{c}{ Coordinates (Angstroms) } \\
Number & & $\mathrm{X}$ & $\mathrm{Y}$ & $\mathrm{Z}$ \\
$-\mathrm{-}$ & & & & \\
1 & $\mathrm{C}$ & 1.499548 & 0.823084 & 0.090454 \\
2 & $\mathrm{C}$ & 0.794881 & -0.345620 & -0.401964 \\
3 & $\mathrm{~N}$ & 0.007506 & -1.217364 & 0.548037 \\
4 & $\mathrm{C}$ & -0.707934 & -0.383021 & -0.412447 \\
5 & $\mathrm{H}$ & 0.002799 & -0.770793 & 1.467711 \\
6 & $\mathrm{C}$ & -1.410095 & 0.798380 & 0.049896 \\
7 & $\mathrm{H}$ & -1.216257 & -0.927003 & -1.206813 \\
8 & $\mathrm{H}$ & 1.308326 & -0.935744 & -1.157619 \\
9 & $\mathrm{H}$ & 1.026307 & 1.492995 & 0.803139 \\
10 & $\mathrm{H}$ & 2.525510 & 1.022737 & -0.195223 \\
11 & $\mathrm{H}$ & -2.430898 & 0.785562 & -0.329206 \\
12 & $\mathrm{H}$ & -1.427322 & 0.807559 & 1.138721 \\
13 & $\mathrm{H}$ & -0.899412 & 1.689293 & -0.312593
\end{tabular}


trans- $C_{1}$-methyl 2-aziridinylmethyl radical

\begin{tabular}{crrrr} 
Center & Atomic & \multicolumn{3}{c}{ Coordinates (Angstroms) } \\
Number & & X & Y & Z \\
-----------150 & \\
1 & $\mathrm{C}$ & -1.947414 & -0.452867 & 0.153085 \\
2 & $\mathrm{C}$ & -0.647459 & -0.176346 & -0.428770 \\
3 & $\mathrm{~N}$ & 0.010312 & 1.167742 & -0.221005 \\
4 & $\mathrm{C}$ & 0.561335 & -0.006329 & 0.448654 \\
5 & $\mathrm{H}$ & -0.558013 & 1.707552 & 0.435395 \\
6 & $\mathrm{H}$ & 0.423940 & -0.126288 & 1.522999 \\
7 & $\mathrm{C}$ & 1.853254 & -0.443804 & -0.043343 \\
8 & $\mathrm{H}$ & -0.478076 & -0.556023 & -1.433777 \\
9 & $\mathrm{H}$ & -2.169148 & -0.155772 & 1.174470 \\
10 & $\mathrm{H}$ & -2.739218 & -0.907619 & -0.430280 \\
11 & $\mathrm{H}$ & 2.532300 & -0.585720 & 0.796107 \\
12 & $\mathrm{H}$ & 1.737830 & -1.384391 & -0.579900 \\
13 & $\mathrm{H}$ & 2.259907 & 0.310141 & -0.715743
\end{tabular}

cis- $C_{1}$-formyl 2-aziridinylmethyl radical

\begin{tabular}{ccrcc} 
Center & Atomic & \multicolumn{3}{c}{ Coordinates (Angstroms) } \\
Number & & $\mathrm{X}$ & $\mathrm{Y}$ & $\mathrm{Z}$ \\
$-\mathrm{-}$ & $\mathrm{C}$ & 1.386233 & 1.215327 & 0.000911 \\
1 & $\mathrm{C}$ & 1.224359 & -0.206316 & -0.239153 \\
2 & $\mathrm{~N}$ & 0.511092 & -1.073631 & 0.771497 \\
3 & $\mathrm{C}$ & -0.137226 & -0.785807 & -0.504180 \\
4 & $\mathrm{H}$ & 0.126185 & -0.469675 & 1.501108 \\
5 & $\mathrm{C}$ & -1.258880 & 0.132176 & -0.545388 \\
6 & $\mathrm{H}$ & -0.226228 & -1.636159 & -1.178202 \\
7 & $\mathrm{H}$ & 2.074150 & -0.720646 & -0.682103 \\
8 & $\mathrm{H}$ & 0.560776 & 1.807126 & 0.386984 \\
9 & $\mathrm{H}$ & 2.341902 & 1.704805 & -0.144617 \\
10 & $\mathrm{O}$ & -1.761038 & 0.528759 & 0.493342 \\
11 & $\mathrm{H}$ & -1.653044 & 0.467614 & -1.503530 \\
12 & & & &
\end{tabular}

trans- $C_{1}$-formyl 2-aziridinylmethyl radical

\begin{tabular}{llcr} 
Center & Atomic & \multicolumn{3}{c}{ Coordinates (Angstroms) } \\
Number & $\mathrm{X}$ & $\mathrm{Y}$ & $\mathrm{Z}$
\end{tabular}




$\begin{array}{rrrrc}1 & \mathrm{~N} & -0.375443 & 1.121223 & 0.357334 \\ 2 & \mathrm{C} & 0.133246 & -0.216423 & 0.651249 \\ 3 & \mathrm{C} & -0.922928 & -0.028172 & -0.415907 \\ 4 & \mathrm{C} & -2.325381 & -0.366094 & -0.210682 \\ 5 & \mathrm{H} & -2.824033 & -0.072448 & 0.706371 \\ 6 & \mathrm{H} & -2.856730 & -0.986851 & -0.923328 \\ 7 & \mathrm{H} & -0.552739 & -0.158723 & -1.433644 \\ 8 & \mathrm{H} & 0.291867 & 1.634764 & -0.218955 \\ 9 & \mathrm{H} & -0.159967 & -0.616566 & 1.619157 \\ 10 & \mathrm{C} & 1.465282 & -0.591988 & 0.218661 \\ 11 & \mathrm{O} & 2.090048 & 0.136449 & -0.534630 \\ 12 & \mathrm{H} & 1.908006 & -1.524269 & 0.566177\end{array}$

cis- $C_{1}$-acetyl 2-aziridinylmethyl radical

\begin{tabular}{|c|c|c|c|c|}
\hline \multirow{2}{*}{$\begin{array}{l}\text { Center } \\
\text { Number }\end{array}$} & \multirow[t]{2}{*}{ Atomic } & \multicolumn{3}{|c|}{ Coordinates (Angstroms) } \\
\hline & & $\mathrm{X}$ & $\mathrm{Y}$ & $\mathrm{Z}$ \\
\hline 1 & $\mathrm{~N}$ & 1.174484 & -0.909854 & 0.894239 \\
\hline 2 & $\mathrm{C}$ & 0.078330 & -0.881758 & -0.063587 \\
\hline 3 & $\mathrm{C}$ & 1.497146 & -0.326224 & -0.415270 \\
\hline 4 & $\mathrm{C}$ & 1.765176 & 1.081871 & -0.596504 \\
\hline 5 & $\mathrm{C}$ & -1.031461 & 0.077294 & 0.134815 \\
\hline 6 & $\mathrm{H}$ & -0.185699 & -1.838880 & -0.510811 \\
\hline 7 & $\mathrm{H}$ & 1.015476 & -0.150538 & 1.564969 \\
\hline 8 & $\mathrm{H}$ & 2.066947 & -1.005780 & -1.046403 \\
\hline 9 & $\mathrm{H}$ & 1.269194 & 1.828218 & 0.012459 \\
\hline 10 & $\mathrm{H}$ & 2.433216 & 1.405603 & -1.385829 \\
\hline 11 & $\mathrm{C}$ & -2.224155 & -0.177270 & -0.649517 \\
\hline 12 & $\mathrm{O}$ & -0.970314 & 1.026234 & 0.897992 \\
\hline 13 & $\mathrm{H}$ & -3.072916 & 0.331702 & -0.195122 \\
\hline 14 & $\mathrm{H}$ & -2.078312 & 0.193939 & -1.662855 \\
\hline 15 & $\mathrm{H}$ & -2.416993 & -1.248637 & -0.679634 \\
\hline
\end{tabular}

trans- $C_{1}$-acetyl 2-aziridinylmethyl radical

\begin{tabular}{ccccc} 
Center & Atomic & \multicolumn{3}{c}{ Coordinates (Angstroms) } \\
Number & & \multicolumn{2}{c}{ X } & $\mathrm{Z}$ \\
- \hdashline & & -1.031764 & 0.919857 & 0.789002 \\
1 & $\mathrm{~N}$ & -0.139462 & -0.208289 & 0.557784 \\
3 & $\mathrm{C}$ & -1.349624 & 0.117702 & -0.388199 \\
4 & $\mathrm{C}$ & -2.540918 & -0.697932 & -0.373079
\end{tabular}




$\begin{array}{rrrrr}5 & \mathrm{H} & -3.053541 & -0.893195 & 0.562247 \\ 6 & \mathrm{H} & -2.872411 & -1.203174 & -1.273168 \\ 7 & \mathrm{H} & -1.012999 & 0.494070 & -1.354032 \\ 8 & \mathrm{H} & -0.243243 & -1.053667 & 1.232906 \\ 9 & \mathrm{C} & 1.194518 & 0.055954 & -0.021940 \\ 10 & \mathrm{H} & -0.532043 & 1.762470 & 0.489644 \\ 11 & \mathrm{C} & 2.152869 & -1.029000 & 0.061365 \\ 12 & \mathrm{O} & 1.481740 & 1.115715 & -0.555356 \\ 13 & \mathrm{H} & 3.160529 & -0.633075 & -0.056053 \\ 14 & \mathrm{H} & 2.065304 & -1.517038 & 1.030938 \\ 15 & \mathrm{H} & 1.952529 & -1.751724 & -0.728227\end{array}$

cis- $C_{1}$-carboxyl 2-aziridinylmethyl radical

\begin{tabular}{|c|c|c|c|c|}
\hline \multirow{2}{*}{$\begin{array}{l}\text { Center } \\
\text { Number }\end{array}$} & \multirow[t]{2}{*}{ Atomic } & \multicolumn{3}{|c|}{ Coordinates (Angstroms) } \\
\hline & & $\mathrm{X}$ & $\mathrm{Y}$ & $\mathrm{Z}$ \\
\hline 1 & $\mathrm{~N}$ & 1.213227 & -1.220006 & 0.007699 \\
\hline 2 & $\mathrm{C}$ & 0.147293 & -0.550761 & -0.724195 \\
\hline 3 & $\mathrm{C}$ & 1.504888 & 0.158585 & -0.409395 \\
\hline 4 & $\mathrm{C}$ & 1.620089 & 1.249640 & 0.530681 \\
\hline 5 & $\mathrm{C}$ & -1.042224 & -0.125134 & -0.012583 \\
\hline 6 & $\mathrm{H}$ & 0.003266 & -0.874049 & -1.754022 \\
\hline 7 & $\mathrm{H}$ & 0.952792 & -1.217679 & 0.999612 \\
\hline 8 & $\mathrm{H}$ & 2.163512 & 0.202565 & -1.274889 \\
\hline 9 & $\mathrm{H}$ & 1.028007 & 1.270789 & 1.437797 \\
\hline 10 & $\mathrm{H}$ & 2.269459 & 2.087520 & 0.306202 \\
\hline 11 & $\mathrm{O}$ & -1.952899 & 0.742425 & -0.627411 \\
\hline 12 & $\mathrm{O}$ & -1.249471 & -0.523035 & 1.121932 \\
\hline 13 & $\mathrm{H}$ & -2.670947 & 0.921793 & -0.031816 \\
\hline
\end{tabular}

trans- $C_{1}$-carboxyl 2-aziridinylmethyl radical

\begin{tabular}{|c|c|c|c|c|}
\hline \multirow{2}{*}{$\begin{array}{l}\text { Center } \\
\text { Number }\end{array}$} & \multirow[t]{2}{*}{ Atomic } & \multicolumn{3}{|c|}{ Coordinates (Angstroms) } \\
\hline & & $\mathrm{X}$ & $\mathrm{Y}$ & $\mathrm{Z}$ \\
\hline 1 & $\mathrm{~N}$ & -1.003243 & 1.083145 & 0.534398 \\
\hline 2 & $\mathrm{C}$ & -0.149735 & -0.094888 & 0.612765 \\
\hline 3 & $\mathrm{C}$ & -1.318779 & 0.032045 & -0.427846 \\
\hline 4 & $\mathrm{C}$ & -2.541142 & -0.718347 & -0.262288 \\
\hline 5 & $\mathrm{H}$ & -3.087418 & -0.667046 & 0.672963 \\
\hline 6 & $\mathrm{H}$ & -2.866507 & -1.414383 & -1.027134 \\
\hline 7 & $\mathrm{H}$ & -0.940311 & 0.154637 & -1.442507 \\
\hline
\end{tabular}




$\begin{array}{rrrrr}8 & \mathrm{H} & -0.305793 & -0.749648 & 1.466087 \\ 9 & \mathrm{C} & 1.183503 & -0.021006 & 0.047505 \\ 10 & \mathrm{H} & -0.462501 & 1.813092 & 0.060997 \\ 11 & \mathrm{O} & 2.030852 & -1.133973 & 0.104984 \\ 12 & \mathrm{O} & 1.566857 & 1.011027 & -0.478182 \\ 13 & \mathrm{H} & 2.860478 & -0.921925 & -0.306424\end{array}$

cis- $C_{1}$-acylamino 2-aziridinylmethyl radical

$\begin{array}{rrrrc}1 & \mathrm{~N} & 1.213801 & -1.212931 & -0.153439 \\ 2 & \mathrm{C} & 0.124996 & -0.461570 & -0.761495 \\ 3 & \mathrm{C} & 1.498230 & 0.203966 & -0.419991 \\ 4 & \mathrm{C} & 1.656428 & 1.179086 & 0.634300 \\ 5 & \mathrm{C} & -1.033610 & -0.118427 & 0.039994 \\ 6 & \mathrm{H} & -0.060455 & -0.663638 & -1.815335 \\ 7 & \mathrm{H} & 0.991558 & -1.324484 & 0.841492 \\ 8 & \mathrm{H} & 2.123503 & 0.346125 & -1.299550 \\ 9 & \mathrm{H} & 1.099664 & 1.096685 & 1.560093 \\ 10 & \mathrm{H} & 2.302158 & 2.035938 & 0.482793 \\ 11 & \mathrm{~N} & -1.988014 & 0.842807 & -0.448620 \\ 12 & \mathrm{O} & -1.199845 & -0.644091 & 1.128315 \\ 13 & \mathrm{H} & -2.012788 & 0.809609 & -1.455768 \\ 14 & \mathrm{H} & -2.901649 & 0.625030 & -0.082684\end{array}$

trans- $C_{1}$-acylamino 2-aziridinylmethyl radical

\begin{tabular}{ccccc} 
Center & Atomic & \multicolumn{3}{c}{ Coordinates (Angstroms) } \\
Number & & X & Y & Z \\
-----------1 & \\
1 & $\mathrm{~N}$ & -0.808455 & 1.070083 & 0.597002 \\
2 & $\mathrm{C}$ & -0.160696 & -0.238567 & 0.533072 \\
3 & $\mathrm{C}$ & -1.288777 & 0.147276 & -0.448624 \\
4 & $\mathrm{C}$ & -2.567721 & -0.522520 & -0.354724 \\
5 & $\mathrm{H}$ & -2.974123 & -0.811172 & 0.610128 \\
6 & $\mathrm{H}$ & -3.120308 & -0.789782 & -1.247194 \\
7 & $\mathrm{H}$ & -0.969915 & 0.444424 & -1.445870 \\
8 & $\mathrm{H}$ & -1.470644 & 1.097980 & 1.373957 \\
9 & $\mathrm{H}$ & -0.403364 & -1.009876 & 1.261685 \\
10 & $\mathrm{C}$ & 1.201119 & -0.262240 & 0.035678 \\
11 & $\mathrm{~N}$ & 1.791969 & 0.935578 & -0.502586 \\
12 & $\mathrm{O}$ & 1.846341 & -1.296977 & 0.073211 \\
13 & $\mathrm{H}$ & 1.392160 & 1.741240 & -0.047475 \\
14 & $\mathrm{H}$ & 2.787326 & 0.919688 & -0.344237
\end{tabular}


cis- $C_{1}$-vinyl 2-aziridinylmethyl radical

\begin{tabular}{|c|c|c|c|c|}
\hline \multirow{2}{*}{$\begin{array}{l}\text { Center } \\
\text { Number }\end{array}$} & \multirow[t]{2}{*}{ Atomic } & \multicolumn{3}{|c|}{ Coordinates (Angstroms) } \\
\hline & & $\mathrm{X}$ & $\mathrm{Y}$ & $\mathrm{Z}$ \\
\hline 1 & $\mathrm{~N}$ & 0.869250 & -1.194060 & 0.562526 \\
\hline 2 & $\mathrm{C}$ & -0.172994 & -0.627031 & -0.287601 \\
\hline 3 & $\mathrm{C}$ & 1.240631 & -0.145295 & -0.459514 \\
\hline 4 & $\mathrm{C}$ & 1.621480 & 1.188549 & -0.034405 \\
\hline 5 & $\mathrm{H}$ & 1.061435 & 1.700676 & 0.743242 \\
\hline 6 & $\mathrm{H}$ & 2.499202 & 1.678383 & -0.439047 \\
\hline 7 & $\mathrm{H}$ & 1.809848 & -0.570080 & -1.283186 \\
\hline 8 & $\mathrm{H}$ & 0.844481 & -0.752442 & 1.484268 \\
\hline 9 & $\mathrm{C}$ & -1.132103 & 0.301031 & 0.279243 \\
\hline 10 & $\mathrm{H}$ & -0.589986 & -1.311593 & -1.024364 \\
\hline 11 & $\mathrm{C}$ & -2.399924 & 0.288990 & -0.138737 \\
\hline 12 & $\mathrm{H}$ & -0.818225 & 1.007859 & 1.045921 \\
\hline 13 & $\mathrm{H}$ & -3.120248 & 0.985997 & 0.286982 \\
\hline 14 & $\mathrm{H}$ & -2.713802 & -0.417838 & -0.905416 \\
\hline
\end{tabular}

trans- $C_{1}$-vinyl 2-aziridinylmethyl radical

\begin{tabular}{crrrr} 
Center & Atomic & \multicolumn{3}{c}{ Coordinates (Angstroms) } \\
Number & & $\mathrm{X}$ & $\mathrm{Y}$ & $\mathrm{Z}$ \\
- & & 0.666400 & 1.236228 & -0.227840 \\
1 & $\mathrm{~N}$ & -168544 & 0.042997 & -0.325715 \\
2 & $\mathrm{C}$ & -0.104490 & -0.029756 & 0.470558 \\
3 & $\mathrm{C}$ & 2.241358 & -0.782972 & -0.024516 \\
4 & $\mathrm{C}$ & 2.364257 & -0.960116 & -1.089492 \\
5 & $\mathrm{H}$ & 1.016017 & 0.055731 & 1.551183 \\
6 & $\mathrm{H}$ & 1.220774 & 1.343433 & -1.080146 \\
7 & $\mathrm{H}$ & -0.207475 & -0.510348 & -1.263679 \\
8 & $\mathrm{H}$ & -1.433785 & 0.119642 & 0.378410 \\
9 & $\mathrm{H}$ & -2.519068 & -0.465484 & -0.133463 \\
10 & $\mathrm{C}$ & -1.498726 & 0.654509 & 1.324782 \\
11 & $\mathrm{C}$ & -3.469307 & -0.407921 & 0.395359 \\
12 & $\mathrm{H}$ & -2.454127 & -1.000352 & -1.079835 \\
13 & $\mathrm{H}$ & &
\end{tabular}

cis- $C_{1}$-ethenyl 2-aziridinylmethyl radical 


\begin{tabular}{|c|c|c|c|c|}
\hline \multirow{2}{*}{$\begin{array}{l}\text { Center } \\
\text { Number }\end{array}$} & \multirow[t]{2}{*}{ Atomic } & \multicolumn{3}{|c|}{ Coordinates (Angstroms) } \\
\hline & & $\mathrm{X}$ & $\mathrm{Y}$ & $\mathrm{Z}$ \\
\hline 1 & $\mathrm{~N}$ & 0.894978 & -1.069912 & 0.662487 \\
\hline 2 & $\mathrm{C}$ & -0.039466 & -0.809435 & -0.428144 \\
\hline 3 & $\mathrm{C}$ & 1.214701 & 0.014675 & -0.339434 \\
\hline 4 & $\mathrm{C}$ & 1.152918 & 1.411207 & 0.048875 \\
\hline 5 & $\mathrm{H}$ & 0.326507 & 1.780662 & 0.649879 \\
\hline 6 & $\mathrm{H}$ & 1.950848 & 2.100171 & -0.201536 \\
\hline 7 & $\mathrm{H}$ & 2.038545 & -0.269117 & -0.990300 \\
\hline 8 & $\mathrm{H}$ & 0.559148 & -0.627862 & 1.521009 \\
\hline 9 & $\mathrm{C}$ & -1.297826 & -0.145432 & -0.148627 \\
\hline 10 & $\mathrm{H}$ & -0.101980 & -1.595211 & -1.179126 \\
\hline 11 & $\mathrm{C}$ & -2.329682 & 0.399051 & 0.080577 \\
\hline 12 & $\mathrm{H}$ & -3.241776 & 0.880338 & 0.283179 \\
\hline
\end{tabular}

trans $-C_{l}$-ethenyl 2-aziridinylmethyl radical

\begin{tabular}{crrrr} 
Center & Atomic & \multicolumn{3}{c}{ Coordinates (Angstroms) } \\
Number & & $\mathrm{X}$ & $\mathrm{Y}$ & $\mathrm{Z}$ \\
- & & -0.576791 & 1.214036 & -0.163027 \\
1 & $\mathrm{~N}$ & 0.157301 & 0.180775 & 0.563561 \\
2 & $\mathrm{C}$ & -0.949389 & -0.209927 & -0.391611 \\
3 & $\mathrm{C}$ & -2.268373 & -0.637276 & 0.056536 \\
4 & $\mathrm{C}$ & -2.779166 & -0.085800 & 0.838095 \\
5 & $\mathrm{H}$ & -2.711367 & -1.553784 & -0.316700 \\
6 & $\mathrm{H}$ & -0.595668 & -0.669908 & -1.315284 \\
7 & $\mathrm{H}$ & -0.033576 & 1.553785 & -0.950191 \\
8 & $\mathrm{H}$ & 0.148784 & 1.633831 \\
9 & $\mathrm{H}$ & 2.518665 & -0.139949 & 0.181036 \\
10 & $\mathrm{C}$ & -0.402943 & -0.132634 \\
11 & $\mathrm{C}$ & 2.621737 & -0.635412 & -0.409898 \\
12 & $\mathrm{H}$ & &
\end{tabular}

cis- $C_{1}$-phenyl 2-aziridinylmethyl radical

\begin{tabular}{|c|c|c|c|c|}
\hline \multirow{2}{*}{$\begin{array}{l}\text { Center } \\
\text { Number }\end{array}$} & \multirow[t]{2}{*}{ Atomic } & \multicolumn{3}{|c|}{ Coordinates (Angstroms) } \\
\hline & & $\mathrm{X}$ & $\mathrm{Y}$ & $\mathrm{Z}$ \\
\hline 1 & $\mathrm{C}$ & 3.452506 & -0.744636 & 0.354785 \\
\hline 2 & $\mathrm{C}$ & 2.173613 & -0.084803 & 0.539059 \\
\hline 3 & $\mathrm{~N}$ & 1.882824 & 1.222694 & -0.159720 \\
\hline
\end{tabular}




$\begin{array}{rrrrr}4 & \mathrm{C} & 1.193850 & 0.012471 & -0.596970 \\ 5 & \mathrm{H} & 2.657899 & 1.429667 & -0.793495 \\ 6 & \mathrm{H} & 1.472052 & -0.452632 & -1.542362 \\ 7 & \mathrm{C} & -0.225932 & -0.025163 & -0.304905 \\ 8 & \mathrm{H} & 1.761756 & -0.103309 & 1.545496 \\ 9 & \mathrm{H} & 3.898401 & -0.817440 & -0.633413 \\ 10 & \mathrm{H} & 4.014437 & -1.127243 & 1.198536 \\ 11 & \mathrm{C} & -0.920462 & 1.163956 & -0.052667 \\ 12 & \mathrm{C} & -2.291286 & 1.127619 & 0.229328 \\ 13 & \mathrm{C} & -2.967580 & -0.097836 & 0.259084 \\ 14 & \mathrm{C} & -2.273050 & -1.286955 & 0.006847 \\ 15 & \mathrm{C} & -0.902226 & -1.250619 & -0.275148 \\ 16 & \mathrm{H} & -0.394402 & 2.117185 & -0.075814 \\ 17 & \mathrm{H} & -2.831532 & 2.052584 & 0.425532 \\ 18 & \mathrm{H} & -4.033886 & -0.126101 & 0.478436 \\ 19 & \mathrm{H} & -2.799110 & -2.240185 & 0.029994 \\ 20 & \mathrm{H} & -0.361980 & -2.175583 & -0.471352\end{array}$

trans- $C_{1}$-phenyl 2-aziridinylmethyl radical

\begin{tabular}{|c|c|c|c|c|}
\hline \multirow{2}{*}{$\begin{array}{l}\text { Center } \\
\text { Number }\end{array}$} & \multirow[t]{2}{*}{ Atomic } & \multicolumn{3}{|c|}{ Coordinates (Angstroms) } \\
\hline & & $\mathrm{X}$ & $\mathrm{Y}$ & $\mathrm{Z}$ \\
\hline 1 & $\mathrm{~N}$ & -2.144058 & 0.814317 & -0.694039 \\
\hline 2 & $\mathrm{C}$ & -1.291445 & -0.362018 & -0.848101 \\
\hline 3 & $\mathrm{C}$ & -2.367474 & -0.343819 & 0.215664 \\
\hline 4 & $\mathrm{C}$ & -2.079226 & -0.149285 & 1.630673 \\
\hline 5 & $\mathrm{H}$ & -1.391049 & 0.629342 & 1.940546 \\
\hline 6 & $\mathrm{H}$ & -2.473453 & -0.830708 & 2.376181 \\
\hline 7 & $\mathrm{H}$ & -3.220170 & -0.987136 & -0.005526 \\
\hline 8 & $\mathrm{H}$ & -2.864486 & 0.826153 & -1.416518 \\
\hline 9 & $\mathrm{C}$ & 0.090896 & -0.171710 & -0.453873 \\
\hline 10 & $\mathrm{H}$ & -1.434811 & -1.027286 & -1.699765 \\
\hline 11 & $\mathrm{C}$ & 0.939600 & -1.277230 & -0.321484 \\
\hline 12 & $\mathrm{C}$ & 2.274274 & -1.093484 & 0.059150 \\
\hline 13 & $\mathrm{C}$ & 2.760244 & 0.195782 & 0.307396 \\
\hline 14 & $\mathrm{C}$ & 1.911540 & 1.301302 & 0.175007 \\
\hline 15 & $\mathrm{C}$ & 0.576866 & 1.117556 & -0.205627 \\
\hline 16 & $\mathrm{H}$ & 0.561585 & -2.280095 & -0.514584 \\
\hline 17 & $\mathrm{H}$ & 2.934445 & -1.953421 & 0.162130 \\
\hline 18 & $\mathrm{H}$ & 3.798430 & 0.338710 & 0.603475 \\
\hline 19 & $\mathrm{H}$ & 2.289556 & 2.304167 & 0.368107 \\
\hline 20 & $\mathrm{H}$ & -0.083304 & 1.977493 & -0.308607 \\
\hline
\end{tabular}


transition state of C-N cleavage of $c i s$ - $C_{1}$-methyl 2-aziridinylmethyl radical

\begin{tabular}{|c|c|c|c|c|}
\hline \multirow{2}{*}{$\begin{array}{l}\text { Center } \\
\text { Number }\end{array}$} & \multirow[t]{2}{*}{ Atomic } & \multicolumn{3}{|c|}{ Coordinates (Angstroms) } \\
\hline & & $\mathrm{X}$ & $\mathrm{Y}$ & $\mathrm{Z}$ \\
\hline 1 & $\mathrm{C}$ & -1.596311 & -0.652419 & 0.212344 \\
\hline 2 & $\mathrm{C}$ & -0.812705 & 0.230203 & -0.519052 \\
\hline 3 & $\mathrm{C}$ & 0.672980 & 0.307642 & -0.417387 \\
\hline 4 & $\mathrm{~N}$ & 0.219602 & 1.309874 & 0.525893 \\
\hline 5 & $\mathrm{C}$ & 1.346910 & -0.886064 & 0.055249 \\
\hline 6 & $\mathrm{H}$ & 1.157994 & 0.764940 & -1.281672 \\
\hline 7 & $\mathrm{H}$ & 0.141163 & 0.860949 & 1.445815 \\
\hline 8 & $\mathrm{H}$ & -1.269860 & 0.841324 & -1.289994 \\
\hline 9 & $\mathrm{H}$ & -1.153061 & -1.244896 & 1.008901 \\
\hline 10 & $\mathrm{H}$ & -2.625559 & -0.832899 & -0.071938 \\
\hline 11 & $\mathrm{H}$ & 2.161568 & -1.135605 & -0.622971 \\
\hline 12 & $\mathrm{H}$ & 1.746632 & -0.706594 & 1.052211 \\
\hline 13 & $\mathrm{H}$ & 0.638673 & -1.712508 & 0.091472 \\
\hline
\end{tabular}

transition state of C-N cleavage of trans- $C_{1}$-methyl 2-aziridinylmethyl radical

\begin{tabular}{|c|c|c|c|c|}
\hline \multirow{2}{*}{$\begin{array}{l}\text { Center } \\
\text { Number }\end{array}$} & \multirow[t]{2}{*}{ Atomic } & \multicolumn{3}{|c|}{ Coordinates (Angstroms) } \\
\hline & & $\mathrm{X}$ & $\mathrm{Y}$ & $\mathrm{Z}$ \\
\hline 1 & $\mathrm{C}$ & -1.959797 & -0.396296 & 0.149968 \\
\hline 2 & $\mathrm{C}$ & -0.678759 & -0.320586 & -0.381502 \\
\hline 3 & $\mathrm{C}$ & 0.522309 & 0.039536 & 0.422899 \\
\hline 4 & $\mathrm{~N}$ & 0.179164 & 1.275005 & -0.253432 \\
\hline 5 & $\mathrm{H}$ & 0.413286 & -0.022153 & 1.510338 \\
\hline 6 & $\mathrm{C}$ & 1.776864 & -0.531902 & -0.026604 \\
\hline 7 & $\mathrm{H}$ & -0.433198 & 1.805817 & 0.378114 \\
\hline 8 & $\mathrm{H}$ & -0.505673 & -0.621250 & -1.408510 \\
\hline 9 & $\mathrm{H}$ & -2.149680 & -0.177357 & 1.197562 \\
\hline 10 & $\mathrm{H}$ & -2.815388 & -0.629734 & -0.474639 \\
\hline 11 & $\mathrm{H}$ & 2.546416 & -0.353052 & 0.722878 \\
\hline 12 & $\mathrm{H}$ & 1.655415 & -1.604094 & -0.173485 \\
\hline 13 & $\mathrm{H}$ & 2.070974 & -0.067728 & -0.966797 \\
\hline
\end{tabular}

transition state of $\mathrm{C}$-N cleavage of $c i s-C_{1}$-formyl 2-aziridinylmethyl radical

\begin{tabular}{llccc} 
Center & Atomic & \multicolumn{3}{c}{ Coordinates (Angstroms) } \\
Number & & $\mathrm{X}$ & $\mathrm{Y}$ & $\mathrm{Z}$
\end{tabular}




$\begin{array}{rrrrr}1 & \mathrm{C} & 1.646662 & -1.025558 & -0.283791 \\ 2 & \mathrm{C} & 1.283777 & 0.153835 & 0.353861 \\ 3 & \mathrm{C} & -0.131165 & 0.562177 & 0.578604 \\ 4 & \mathrm{~N} & 0.265388 & 1.326202 & -0.587830 \\ 5 & \mathrm{C} & -1.129893 & -0.479337 & 0.436159 \\ 6 & \mathrm{H} & -0.312566 & 1.180953 & 1.459607 \\ 7 & \mathrm{H} & 0.001008 & 0.776023 & -1.414271 \\ 8 & \mathrm{H} & 2.048647 & 0.780436 & 0.798212 \\ 9 & \mathrm{H} & 0.897227 & -1.712298 & -0.668730 \\ 10 & \mathrm{H} & 2.688126 & -1.273406 & -0.458678 \\ 11 & \mathrm{O} & -2.013106 & -0.367564 & -0.398013 \\ 12 & \mathrm{H} & -1.091597 & -1.361320 & 1.073778 \\ ------------- & \end{array}$

transition state of C-N cleavage of trans- $C_{1}$-formyl 2-aziridinylmethyl radical

\begin{tabular}{|c|c|c|c|c|}
\hline \multirow{2}{*}{$\begin{array}{l}\text { Center } \\
\text { Number }\end{array}$} & \multirow[t]{2}{*}{ Atomic } & \multicolumn{3}{|c|}{ Coordinates (Angstroms) } \\
\hline & & $\mathrm{X}$ & $\mathrm{Y}$ & $\mathrm{Z}$ \\
\hline 1 & $\mathrm{~N}$ & -0.245615 & 1.366260 & 0.082895 \\
\hline 2 & $\mathrm{C}$ & 0.065069 & 0.078885 & 0.647437 \\
\hline 3 & $\mathrm{C}$ & -0.973178 & -0.267450 & -0.382719 \\
\hline 4 & $\mathrm{C}$ & -2.316808 & -0.451831 & -0.111282 \\
\hline 5 & $\mathrm{H}$ & -2.720997 & -0.291833 & 0.883016 \\
\hline 6 & $\mathrm{H}$ & -3.013206 & -0.695716 & -0.906502 \\
\hline 7 & $\mathrm{H}$ & -0.584607 & -0.480133 & -1.374025 \\
\hline 8 & $\mathrm{H}$ & -0.263926 & -0.046427 & 1.682078 \\
\hline 9 & $\mathrm{C}$ & 1.419425 & -0.526533 & 0.370122 \\
\hline 10 & $\mathrm{H}$ & 0.422498 & 1.491605 & -0.693092 \\
\hline 11 & $\mathrm{O}$ & 2.122984 & -0.146851 & -0.542727 \\
\hline 12 & $\mathrm{H}$ & 1.728629 & -1.364944 & 1.028728 \\
\hline
\end{tabular}

transition state of C-N cleavage of $c i s$ - $C_{1}$-acetyl 2-aziridinylmethyl radical

\begin{tabular}{|c|c|c|c|c|}
\hline \multirow{2}{*}{$\begin{array}{l}\text { Center } \\
\text { Number }\end{array}$} & \multirow[t]{2}{*}{ Atomic } & \multicolumn{3}{|c|}{ Coordinates (Angstroms) } \\
\hline & & $\mathrm{X}$ & $\mathrm{Y}$ & $\mathrm{Z}$ \\
\hline 1 & $\mathrm{~N}$ & 1.151416 & -1.394929 & 0.318108 \\
\hline 2 & $\mathrm{C}$ & 0.192641 & -0.697608 & -0.498586 \\
\hline 3 & $\mathrm{C}$ & 1.421509 & 0.171130 & -0.612084 \\
\hline 4 & $\mathrm{C}$ & 1.661619 & 1.312313 & 0.130909 \\
\hline 5 & $\mathrm{C}$ & -1.012863 & -0.066911 & 0.151465 \\
\hline 6 & $\mathrm{H}$ & 0.985763 & -1.058552 & 1.278479 \\
\hline
\end{tabular}




$\begin{array}{rrrrr}7 & \mathrm{H} & -0.063096 & -1.217370 & -1.426505 \\ 8 & \mathrm{H} & 2.107123 & -0.114610 & -1.401447 \\ 9 & \mathrm{H} & 2.590124 & 1.858322 & 0.004846 \\ 10 & \mathrm{H} & 0.973094 & 1.655846 & 0.895507 \\ 11 & \mathrm{C} & -2.073662 & 0.345732 & -0.746829 \\ 12 & \mathrm{O} & -1.096337 & 0.113228 & 1.348532 \\ 13 & \mathrm{H} & -3.002997 & 0.447847 & -0.188411 \\ 14 & \mathrm{H} & -1.814933 & 1.302029 & -1.199021 \\ 15 & \mathrm{H} & -2.199754 & -0.402769 & -1.527705\end{array}$

transition state of C-N cleavage of trans- $C_{1}$-acetyl 2-aziridinylmethyl radical

\begin{tabular}{|c|c|c|c|c|}
\hline \multirow{2}{*}{$\begin{array}{l}\text { Center } \\
\text { Number }\end{array}$} & \multirow[t]{2}{*}{ Atomic } & \multicolumn{3}{|c|}{ Coordinates (Angstroms) } \\
\hline & & $\mathrm{X}$ & $\mathrm{Y}$ & $\mathrm{Z}$ \\
\hline 1 & $\mathrm{~N}$ & -0.921482 & 1.251895 & 0.733734 \\
\hline 2 & $\mathrm{C}$ & -0.213533 & 0.009821 & 0.564540 \\
\hline 3 & $\mathrm{C}$ & -1.299778 & -0.099633 & -0.468522 \\
\hline 4 & $\mathrm{C}$ & -2.489631 & -0.780971 & -0.286739 \\
\hline 5 & $\mathrm{H}$ & -2.740668 & -1.238641 & 0.664636 \\
\hline 6 & $\mathrm{H}$ & -3.233601 & -0.814714 & -1.075532 \\
\hline 7 & $\mathrm{H}$ & -1.050312 & 0.308276 & -1.443348 \\
\hline 8 & $\mathrm{H}$ & -0.316418 & -0.681160 & 1.405006 \\
\hline 9 & $\mathrm{C}$ & 1.177542 & 0.042087 & -0.019917 \\
\hline 10 & $\mathrm{H}$ & -0.449888 & 1.921618 & 0.106587 \\
\hline 11 & $\mathrm{O}$ & 1.597238 & 0.998003 & -0.638403 \\
\hline 12 & $\mathrm{C}$ & 1.979291 & -1.152584 & 0.160250 \\
\hline 13 & $\mathrm{H}$ & 3.032143 & -0.906048 & 0.031241 \\
\hline 14 & $\mathrm{H}$ & 1.819458 & -1.549402 & 1.161705 \\
\hline 15 & $\mathrm{H}$ & 1.688412 & -1.899542 & -0.576882 \\
\hline
\end{tabular}

transition state of C-N cleavage of cis- $C_{1}$-carboxyl 2-aziridinylmethyl radical

\begin{tabular}{ccrcc} 
Center & Atomic & \multicolumn{3}{c}{ Coordinates (Angstroms) } \\
Number & & $\mathrm{X}$ & $\mathrm{Y}$ & $\mathrm{Z}$ \\
$-\mathrm{-}$ & $\mathrm{N}$ & -1.122864 & 1.418014 & -0.070527 \\
1 & $\mathrm{C}$ & -0.226652 & 0.491541 & -0.711580 \\
2 & $\mathrm{C}$ & -1.435695 & -0.362916 & -0.418625 \\
3 & $\mathrm{C}$ & -1.571807 & -1.203752 & 0.670621 \\
4 & $\mathrm{C}$ & 1.003412 & 0.113812 & -0.043177 \\
5 & $\mathrm{H}$ & -0.857315 & 1.407605 & 0.925605 \\
6 & $\mathrm{H}$ & -0.074986 & 0.684439 & -1.777589 \\
7 & & & &
\end{tabular}




$\begin{array}{rrrrr}8 & \mathrm{H} & -2.200975 & -0.343514 & -1.185944 \\ 9 & \mathrm{H} & -2.492281 & -1.757789 & 0.818645 \\ 10 & \mathrm{H} & -0.804350 & -1.285851 & 1.432829 \\ 11 & \mathrm{O} & 1.777488 & -0.939538 & -0.544437 \\ 12 & \mathrm{O} & 1.363811 & 0.713918 & 0.956015 \\ 13 & \mathrm{H} & 2.544015 & -1.058142 & 0.004078\end{array}$

transition state of C-N cleavage of trans- $C_{1}$-carboxyl 2-aziridinylmethyl radical

\begin{tabular}{|c|c|c|c|c|}
\hline \multirow{2}{*}{$\begin{array}{l}\text { Center } \\
\text { Number }\end{array}$} & \multirow[t]{2}{*}{ Atomic } & \multicolumn{3}{|c|}{ Coordinates (Angstroms) } \\
\hline & & $X$ & $\mathrm{Y}$ & $\mathrm{Z}$ \\
\hline 1 & $\mathrm{~N}$ & -0.867663 & 1.406880 & 0.278779 \\
\hline 2 & $\mathrm{C}$ & -0.202634 & 0.140852 & 0.523644 \\
\hline 3 & $\mathrm{C}$ & -1.283342 & -0.204996 & -0.436603 \\
\hline 4 & $\mathrm{C}$ & -2.526870 & -0.723610 & -0.081393 \\
\hline 5 & $\mathrm{H}$ & -2.805804 & -0.845214 & 0.960544 \\
\hline 6 & $\mathrm{H}$ & -3.277866 & -0.941440 & -0.833353 \\
\hline 7 & $\mathrm{H}$ & -1.023592 & -0.157401 & -1.490614 \\
\hline 8 & $\mathrm{H}$ & -0.398758 & 1.847750 & -0.523157 \\
\hline 9 & $\mathrm{H}$ & -0.347278 & -0.224020 & 1.542387 \\
\hline 10 & $\mathrm{C}$ & 1.163153 & -0.044222 & 0.073228 \\
\hline 11 & $\mathrm{O}$ & 1.738189 & -1.320592 & 0.087881 \\
\hline 12 & $\mathrm{O}$ & 1.811195 & 0.912325 & -0.318510 \\
\hline 13 & $\mathrm{H}$ & 2.630020 & -1.269851 & -0.235481 \\
\hline
\end{tabular}

transition state of $\mathrm{C}-\mathrm{N}$ cleavage of $c i s-C_{1}$-acylamino 2-aziridinylmethyl radical

\begin{tabular}{crrrr} 
Center & Atomic & \multicolumn{3}{c}{ Coordinates (Angstroms) } \\
Number & & $\mathrm{X}$ & $\mathrm{Y}$ & $\mathrm{Z}$ \\
\hline 1 & $\mathrm{~N}$ & 1.144753 & -1.397239 & 0.144794 \\
2 & $\mathrm{C}$ & 0.184507 & -0.603006 & -0.595936 \\
3 & $\mathrm{C}$ & 1.407560 & 0.247395 & -0.579399 \\
4 & $\mathrm{C}$ & 1.590254 & 1.306146 & 0.300897 \\
5 & $\mathrm{H}$ & 0.837768 & 1.558474 & 1.043553 \\
6 & $\mathrm{H}$ & 2.509170 & 1.882548 & 0.305001 \\
7 & $\mathrm{H}$ & 2.136863 & 0.068028 & -1.361095 \\
8 & $\mathrm{C}$ & -0.975630 & -0.071865 & 0.092883 \\
9 & $\mathrm{H}$ & -0.080066 & -1.035468 & -1.562856 \\
10 & $\mathrm{H}$ & 1.011451 & -1.182183 & 1.140575 \\
11 & $\mathrm{O}$ & -1.058236 & -0.161291 & 1.306793 \\
12 & $\mathrm{~N}$ & -2.030264 & 0.561165 & -0.655862
\end{tabular}




$\begin{array}{lllll}13 & \mathrm{H} & -2.083075 & 0.150483 & -1.574892 \\ 14 & \mathrm{H} & -2.907794 & 0.428940 & -0.177822\end{array}$

transition state of C-N cleavage of trans- $C_{1}$-acylamino 2-aziridinylmethyl radical

\begin{tabular}{|c|c|c|c|c|}
\hline \multirow{2}{*}{$\begin{array}{l}\text { Center } \\
\text { Number }\end{array}$} & \multirow[t]{2}{*}{ Atomic } & \multicolumn{3}{|c|}{ Coordinates (Angstroms) } \\
\hline & & $\mathrm{X}$ & Y & $\mathrm{Z}$ \\
\hline 1 & $\mathrm{~N}$ & 0.703902 & -0.967697 & 0.986190 \\
\hline 2 & $\mathrm{C}$ & 0.197237 & 0.300038 & 0.498650 \\
\hline 3 & $\mathrm{C}$ & 1.233597 & -0.074581 & -0.503813 \\
\hline 4 & $\mathrm{C}$ & 2.519917 & 0.449471 & -0.505404 \\
\hline 5 & $\mathrm{H}$ & 2.839587 & 1.162072 & 0.250542 \\
\hline 6 & $\mathrm{H}$ & 3.257916 & 0.127555 & -1.232308 \\
\hline 7 & $\mathrm{H}$ & 0.916909 & -0.720924 & -1.314401 \\
\hline 8 & $\mathrm{H}$ & 0.388873 & 1.209662 & 1.076644 \\
\hline 9 & $\mathrm{C}$ & -1.176879 & 0.231455 & 0.040829 \\
\hline 10 & $\mathrm{H}$ & 1.458287 & -0.756659 & 1.650994 \\
\hline 11 & $\mathrm{O}$ & -1.872952 & 1.233368 & 0.047973 \\
\hline 12 & $\mathrm{~N}$ & -1.719924 & -1.019243 & -0.422266 \\
\hline 13 & $\mathrm{H}$ & -2.425935 & -0.841426 & -1.119400 \\
\hline 14 & $\mathrm{H}$ & -0.983094 & -1.576943 & -0.824902 \\
\hline
\end{tabular}

transition state of C-N cleavage of $c i s$ - $C_{1}$-vinyl 2-aziridinylmethyl radical

\begin{tabular}{crrrr} 
Center & Atomic & \multicolumn{3}{c}{ Coordinates (Angstroms) } \\
Number & & X & Y & $\mathrm{Z}$ \\
-1 & $\mathrm{~N}$ & -0.736481 & 1.378237 & 0.527412 \\
2 & $\mathrm{C}$ & 0.136845 & 0.579556 & -0.309826 \\
3 & $\mathrm{C}$ & -1.213567 & 0.000371 & -0.555278 \\
4 & $\mathrm{C}$ & -1.670718 & -1.159062 & 0.057894 \\
5 & $\mathrm{H}$ & -1.054741 & -1.700308 & 0.771383 \\
6 & $\mathrm{H}$ & -2.673320 & -1.532550 & -0.120876 \\
7 & $\mathrm{H}$ & -1.815907 & 0.475072 & -1.321404 \\
8 & $\mathrm{C}$ & 1.107509 & -0.298020 & 0.314811 \\
9 & $\mathrm{H}$ & 0.571183 & 1.139971 & -1.140033 \\
10 & $\mathrm{H}$ & -0.745561 & 0.942428 & 1.457781 \\
11 & $\mathrm{C}$ & 2.359599 & -0.344030 & -0.146034 \\
12 & $\mathrm{H}$ & 0.815143 & -0.919578 & 1.159861 \\
13 & $\mathrm{H}$ & 3.088601 & -1.003119 & 0.323091 \\
14 & $\mathrm{H}$ & 2.651965 & 0.277529 & -0.991083
\end{tabular}


transition state of C-N cleavage of trans- $C_{1}$-vinyl 2-aziridinylmethyl radical

\begin{tabular}{crrrr} 
Center & Atomic & \multicolumn{3}{c}{ Coordinates (Angstroms) } \\
Number & & $\mathrm{X}$ & $\mathrm{Y}$ & $\mathrm{Z}$ \\
\hline 1 & $\mathrm{~N}$ & 0.555703 & 1.399949 & -0.102718 \\
2 & $\mathrm{C}$ & -0.129977 & 0.139375 & -0.308523 \\
3 & $\mathrm{C}$ & 1.082827 & -0.241552 & 0.468210 \\
4 & $\mathrm{C}$ & 2.224672 & -0.778486 & -0.112429 \\
5 & $\mathrm{H}$ & 2.291196 & -0.935198 & -1.185956 \\
6 & $\mathrm{H}$ & 3.101235 & -1.013091 & 0.481928 \\
7 & $\mathrm{H}$ & 1.012866 & -0.170504 & 1.547637 \\
8 & $\mathrm{H}$ & -0.204495 & -0.268946 & -1.321408 \\
9 & $\mathrm{C}$ & -1.389583 & 0.047826 & 0.403875 \\
10 & $\mathrm{H}$ & 1.157939 & 1.554014 & -0.920748 \\
11 & $\mathrm{C}$ & -2.455088 & -0.493495 & -0.191017 \\
12 & $\mathrm{H}$ & -1.466426 & 0.420641 & 1.424181 \\
13 & $\mathrm{H}$ & -3.401096 & -0.562252 & 0.344018 \\
14 & $\mathrm{H}$ & -2.378245 & -0.866310 & -1.211323
\end{tabular}

transition state of C-N cleavage of $c i s$ - $C_{l}$-ethenyl 2-aziridinylmethyl radical

\begin{tabular}{|c|c|c|c|c|}
\hline \multirow{2}{*}{$\begin{array}{l}\text { Center } \\
\text { Number }\end{array}$} & \multirow[t]{2}{*}{ Atomic } & \multicolumn{3}{|c|}{ Coordinates (Angstroms) } \\
\hline & & $X$ & $\mathrm{Y}$ & $\mathrm{Z}$ \\
\hline 1 & $\mathrm{~N}$ & 0.777183 & -1.273048 & 0.648309 \\
\hline 2 & $\mathrm{C}$ & -0.013899 & -0.745376 & -0.445907 \\
\hline 3 & $\mathrm{C}$ & 1.195198 & 0.124817 & -0.433163 \\
\hline 4 & $\mathrm{C}$ & 1.221695 & 1.387716 & 0.144448 \\
\hline 5 & $\mathrm{H}$ & 0.345307 & 1.801297 & 0.636727 \\
\hline 6 & $\mathrm{H}$ & 2.130854 & 1.979105 & 0.163153 \\
\hline 7 & $\mathrm{H}$ & 2.057239 & -0.229962 & -0.986444 \\
\hline 8 & $\mathrm{C}$ & -1.276443 & -0.093784 & -0.156234 \\
\hline 9 & $\mathrm{H}$ & -0.095783 & -1.432088 & -1.290702 \\
\hline 10 & $\mathrm{H}$ & 0.460032 & -0.803196 & 1.505194 \\
\hline 11 & $\mathrm{C}$ & -2.311729 & 0.440522 & 0.081298 \\
\hline 12 & $\mathrm{H}$ & -3.226856 & 0.912814 & 0.291260 \\
\hline
\end{tabular}

transition state of $\mathrm{C}-\mathrm{N}$ cleavage of trans- $C_{1}$-ethynyl 2-aziridinylmethyl radical

\begin{tabular}{llcc} 
Center & Atomic & \multicolumn{3}{c}{ Coordinates (Angstroms) } \\
Number & & $\mathrm{X}$ & $\mathrm{Y}$
\end{tabular}




$\begin{array}{rrrrc}1 & \mathrm{~N} & -0.467365 & 1.388810 & -0.114876 \\ 2 & \mathrm{C} & 0.118397 & 0.209545 & 0.494441 \\ 3 & \mathrm{C} & -0.962949 & -0.335484 & -0.367677 \\ 4 & \mathrm{C} & -2.240769 & -0.670422 & 0.075595 \\ 5 & \mathrm{H} & -2.545196 & -0.481792 & 1.100275 \\ 6 & \mathrm{H} & -2.989361 & -1.051246 & -0.611043 \\ 7 & \mathrm{H} & -0.681754 & -0.597477 & -1.384025 \\ 8 & \mathrm{H} & 0.040872 & 1.563713 & -0.991485 \\ 9 & \mathrm{H} & -0.065706 & 0.151625 & 1.568984 \\ 10 & \mathrm{C} & 1.479080 & -0.163046 & 0.159454 \\ 11 & \mathrm{C} & 2.594841 & -0.468570 & -0.115235 \\ 12 & \mathrm{H} & 3.581102 & -0.738634 & -0.358042 \\ ----------1 & \end{array}$

transition state of $\mathrm{C}$-N cleavage of $c i s$ - $C_{1}$-phenyl 2-aziridinylmethyl radical

\begin{tabular}{|c|c|c|c|c|}
\hline \multirow{2}{*}{$\begin{array}{l}\text { Center } \\
\text { Number }\end{array}$} & \multirow[t]{2}{*}{ Atomic } & \multicolumn{3}{|c|}{ Coordinates (Angstroms) } \\
\hline & & $X$ & $\mathrm{Y}$ & $\mathrm{Z}$ \\
\hline 1 & $\mathrm{C}$ & -3.402214 & -0.810435 & -0.251066 \\
\hline 2 & $\mathrm{C}$ & -2.131404 & -0.311248 & -0.506225 \\
\hline 3 & $\mathrm{C}$ & -1.219145 & 0.191258 & 0.558943 \\
\hline 4 & $\mathrm{~N}$ & -1.795014 & 1.399525 & 0.002146 \\
\hline 5 & $\mathrm{H}$ & -1.468761 & -0.095493 & 1.585433 \\
\hline 6 & $\mathrm{C}$ & 0.199241 & 0.054626 & 0.290591 \\
\hline 7 & $\mathrm{H}$ & -2.621540 & 1.629300 & 0.567529 \\
\hline 8 & $\mathrm{H}$ & -1.726695 & -0.362888 & -1.510540 \\
\hline 9 & $\mathrm{H}$ & -3.802561 & -0.844432 & 0.758889 \\
\hline 10 & $\mathrm{H}$ & -4.050890 & -1.136991 & -1.056823 \\
\hline 11 & $\mathrm{C}$ & 0.976328 & 1.191187 & 0.036892 \\
\hline 12 & $\mathrm{C}$ & 2.345805 & 1.059266 & -0.222207 \\
\hline 13 & $\mathrm{C}$ & 2.938193 & -0.209216 & -0.227605 \\
\hline 14 & $\mathrm{C}$ & 2.161106 & -1.345777 & 0.026094 \\
\hline 15 & $\mathrm{C}$ & 0.791629 & -1.213856 & 0.285193 \\
\hline 16 & $\mathrm{H}$ & 0.515535 & 2.177885 & 0.041091 \\
\hline 17 & $\mathrm{H}$ & 2.950268 & 1.943349 & -0.419549 \\
\hline 18 & $\mathrm{H}$ & 4.003450 & -0.311831 & -0.429147 \\
\hline 19 & $\mathrm{H}$ & 2.621899 & -2.332475 & 0.021895 \\
\hline 20 & $\mathrm{H}$ & 0.187166 & -2.097938 & 0.482535 \\
\hline
\end{tabular}

transition state of C-N cleavage of trans- $C_{1}$-phenyl 2-aziridinylmethyl radical

Center Atomic Coordinates (Angstroms)




\begin{tabular}{rrrrr} 
Number & & \multicolumn{1}{c}{ X } & Y & Z \\
-1 & N & -2.074161 & 1.072628 & -0.628022 \\
2 & $\mathrm{C}$ & -1.339508 & -0.156781 & -0.860158 \\
3 & $\mathrm{C}$ & -2.292026 & -0.511106 & 0.224635 \\
4 & $\mathrm{C}$ & -1.983854 & -0.515674 & 1.583497 \\
5 & $\mathrm{H}$ & -1.013032 & -0.184436 & 1.938477 \\
6 & $\mathrm{H}$ & -2.732151 & -0.771371 & 2.326168 \\
7 & $\mathrm{H}$ & -3.253437 & -0.907760 & -0.090052 \\
8 & $\mathrm{H}$ & -2.893471 & 1.060413 & -1.249249 \\
9 & $\mathrm{C}$ & 0.051443 & -0.065606 & -0.460852 \\
10 & $\mathrm{H}$ & -1.455671 & -0.679770 & -1.814498 \\
11 & $\mathrm{C}$ & 0.872611 & -1.197896 & -0.520876 \\
12 & $\mathrm{C}$ & 2.215597 & -1.109866 & -0.135340 \\
13 & $\mathrm{C}$ & 2.737415 & 0.110455 & 0.310220 \\
14 & $\mathrm{C}$ & 1.916247 & 1.242745 & 0.370244 \\
15 & $\mathrm{C}$ & 0.573261 & 1.154714 & -0.015293 \\
16 & $\mathrm{H}$ & 0.466710 & -2.147131 & -0.867458 \\
17 & $\mathrm{H}$ & 2.854348 & -1.990625 & -0.182029 \\
18 & $\mathrm{H}$ & 3.782067 & 0.178930 & 0.610112 \\
19 & $\mathrm{H}$ & 2.322147 & 2.191980 & 0.716826 \\
20 & $\mathrm{H}$ & -0.065491 & 2.035474 & 0.031397 \\
& & & &
\end{tabular}

transition state of C-C cleavage of trans- $C_{1}$-methyl 2-aziridinylmethyl radical

\begin{tabular}{ccrrc} 
Center & Atomic & \multicolumn{3}{c}{ Coordinates (Angstroms) } \\
Number & & X & Y & Z \\
-1 & $\mathrm{C}$ & 1.685351 & 0.695579 & 0.040380 \\
2 & $\mathrm{C}$ & 0.883231 & -0.430363 & -0.397065 \\
3 & $\mathrm{C}$ & -0.912866 & -0.523240 & -0.323728 \\
4 & $\mathrm{~N}$ & 0.021768 & -0.866045 & 0.671428 \\
5 & $\mathrm{C}$ & -1.454146 & 0.793698 & -0.049520 \\
6 & $\mathrm{H}$ & -1.250856 & -1.424376 & -0.833275 \\
7 & $\mathrm{H}$ & -0.078848 & -0.123676 & 1.345838 \\
8 & $\mathrm{H}$ & 1.240930 & -1.147142 & -1.134764 \\
9 & $\mathrm{H}$ & 2.056697 & 1.236248 & -0.828931 \\
10 & $\mathrm{H}$ & 1.439241 & 0.937057 & 1.073356 \\
11 & $\mathrm{H}$ & -2.176848 & 1.056860 & -0.820473 \\
12 & $\mathrm{H}$ & -1.945549 & 0.789054 & 0.922294 \\
13 & $\mathrm{H}$ & -0.646560 & 1.524246 & -0.044441
\end{tabular}

transition state of C-C cleavage of trans- $C_{1}$-methyl 2-aziridinylmethyl radical 


\begin{tabular}{ccrcc} 
Center & Atomic & \multicolumn{3}{c}{ Coordinates (Angstroms) } \\
Number & & X & Y & $\mathrm{Z}$ \\
-1 & $\mathrm{C}$ & -2.095303 & -0.233493 & 0.148058 \\
2 & $\mathrm{C}$ & -0.719718 & -0.467775 & -0.246120 \\
3 & $\mathrm{C}$ & 0.728554 & 0.260277 & 0.536453 \\
4 & $\mathrm{~N}$ & -0.060715 & 0.786443 & -0.503544 \\
5 & $\mathrm{H}$ & 0.439221 & 0.717373 & 1.481583 \\
6 & $\mathrm{C}$ & 1.928475 & -0.336026 & -0.017725 \\
7 & $\mathrm{H}$ & -0.567882 & 1.531378 & -0.051971 \\
8 & $\mathrm{H}$ & -0.413689 & -1.364056 & -0.783659 \\
9 & $\mathrm{H}$ & -2.477127 & -1.108950 & 0.671229 \\
10 & $\mathrm{H}$ & -2.294171 & 0.837183 & 0.153005 \\
11 & $\mathrm{H}$ & 2.685398 & -0.419104 & 0.760790 \\
12 & $\mathrm{H}$ & 1.695840 & -1.326876 & -0.405049 \\
13 & $\mathrm{H}$ & 2.305368 & 0.290056 & -0.825124 \\
& & & &
\end{tabular}

transition state of C-C cleavage of $c i s$ - $C_{1}$-formyl 2-aziridinylmethyl radical

\begin{tabular}{|c|c|c|c|c|}
\hline \multirow{2}{*}{$\begin{array}{l}\text { Center } \\
\text { Number }\end{array}$} & \multirow[t]{2}{*}{ Atomic } & \multicolumn{3}{|c|}{ Coordinates (Angstroms) } \\
\hline & & $X$ & $\mathrm{Y}$ & $\mathrm{Z}$ \\
\hline 1 & $\mathrm{C}$ & -1.613035 & -1.069366 & -0.154947 \\
\hline 2 & $\mathrm{C}$ & -1.291565 & 0.343559 & -0.207880 \\
\hline 3 & $\mathrm{C}$ & 0.369724 & 1.034691 & -0.257141 \\
\hline 4 & $\mathrm{~N}$ & -0.399340 & 0.686345 & 0.869175 \\
\hline 5 & $\mathrm{C}$ & 1.323816 & -0.020029 & -0.539584 \\
\hline 6 & $\mathrm{H}$ & 0.310953 & 2.109654 & -0.421216 \\
\hline 7 & $\mathrm{H}$ & 0.054657 & -0.147530 & 1.207706 \\
\hline 8 & $\mathrm{H}$ & -1.990521 & 1.084687 & -0.592731 \\
\hline 9 & $\mathrm{H}$ & -1.976207 & -1.395728 & -1.128351 \\
\hline 10 & $\mathrm{H}$ & -1.099383 & -1.527134 & 0.689167 \\
\hline 11 & $\mathrm{O}$ & 1.623694 & -0.820480 & 0.330904 \\
\hline 12 & $\mathrm{H}$ & 1.772694 & -0.097658 & -1.528727 \\
\hline
\end{tabular}

transition state of C-C cleavage of trans- $C_{l}$-formyl 2-aziridinylmethyl radical

\begin{tabular}{|c|c|c|c|c|}
\hline \multirow{2}{*}{$\begin{array}{l}\text { Center } \\
\text { Number }\end{array}$} & \multirow[t]{2}{*}{ Atomic } & \multicolumn{3}{|c|}{ Coordinates (Angstroms) } \\
\hline & & $X$ & $\mathrm{Y}$ & $\mathrm{Z}$ \\
\hline 1 & $\mathrm{~N}$ & 0.412432 & -0.437307 & 0.966893 \\
\hline 2 & $\mathrm{C}$ & -0.294480 & 0.683585 & 0.440444 \\
\hline
\end{tabular}




$\begin{array}{rrrrr}3 & \mathrm{C} & 1.087194 & -0.354521 & -0.306818 \\ 4 & \mathrm{C} & 2.376392 & 0.115396 & -0.411052 \\ 5 & \mathrm{H} & 2.911738 & 0.460156 & 0.467350 \\ 6 & \mathrm{H} & 2.850022 & 0.219327 & -1.381421 \\ 7 & \mathrm{H} & 0.597600 & -0.793338 & -1.177726 \\ 8 & \mathrm{H} & -0.173551 & -1.267465 & 1.034508 \\ 9 & \mathrm{H} & 0.030507 & 1.667348 & 0.756875 \\ 10 & \mathrm{C} & -1.573142 & 0.538263 & -0.227692 \\ 11 & \mathrm{O} & -2.074104 & -0.567130 & -0.352367 \\ 12 & \mathrm{H} & -2.086290 & 1.415819 & -0.618197\end{array}$

transition state of C-C cleavage of $\operatorname{cis}$ - $C_{1}$-acetyl 2-aziridinylmethyl radical

\begin{tabular}{ccccc} 
Center & Atomic & \multicolumn{3}{c}{ Coordinates (Angstroms) } \\
Number & & X & Y & $\mathrm{Z}$ \\
- & & & & \\
1 & $\mathrm{~N}$ & 1.122351 & -0.890423 & 0.870653 \\
2 & $\mathrm{C}$ & -0.010711 & -0.913700 & -0.008314 \\
3 & $\mathrm{C}$ & 1.614426 & -0.328609 & -0.365717 \\
4 & $\mathrm{C}$ & 1.854077 & 1.026159 & -0.595825 \\
5 & $\mathrm{H}$ & 1.450276 & 1.790626 & 0.058172 \\
6 & $\mathrm{H}$ & 2.396245 & 1.335358 & -1.482503 \\
7 & $\mathrm{H}$ & 2.082899 & -1.063077 & -1.016760 \\
8 & $\mathrm{H}$ & 0.972027 & -0.140401 & 1.552368 \\
9 & $\mathrm{H}$ & -0.214815 & -1.848911 & -0.523030 \\
10 & $\mathrm{C}$ & -1.061907 & 0.082867 & 0.127137 \\
11 & $\mathrm{C}$ & -2.261632 & -0.156897 & -0.651113 \\
12 & $\mathrm{O}$ & -0.957835 & 1.068901 & 0.852250 \\
13 & $\mathrm{H}$ & -3.088164 & 0.412640 & -0.228746 \\
14 & $\mathrm{H}$ & -2.094637 & 0.155131 & -1.681002 \\
15 & $\mathrm{H}$ & -2.503130 & -1.218532 & -0.628083 \\
----------- &
\end{tabular}

transition state of C-C cleavage of trans- $C_{1}$-acetyl 2-aziridinylmethyl radical

\begin{tabular}{|c|c|c|c|c|}
\hline \multirow{2}{*}{$\begin{array}{l}\text { Center } \\
\text { Number }\end{array}$} & \multirow[t]{2}{*}{ Atomic } & \multicolumn{3}{|c|}{ Coordinates (Angstroms) } \\
\hline & & $\mathrm{X}$ & $\mathrm{Y}$ & $\mathrm{Z}$ \\
\hline 1 & $\mathrm{~N}$ & -1.018066 & 0.831296 & 0.812281 \\
\hline 2 & $\mathrm{C}$ & -0.063062 & -0.223603 & 0.627200 \\
\hline 3 & $\mathrm{C}$ & -1.457149 & 0.171671 & -0.396112 \\
\hline 4 & $\mathrm{C}$ & -2.556234 & -0.682974 & -0.390164 \\
\hline 5 & $\mathrm{H}$ & -3.061421 & -0.926284 & 0.538511 \\
\hline 6 & $\mathrm{H}$ & -2.874510 & -1.175383 & -1.302300 \\
\hline
\end{tabular}




$\begin{array}{rrrrr}7 & \mathrm{H} & -1.038816 & 0.515191 & -1.340675 \\ 8 & \mathrm{H} & -0.204469 & -1.128359 & 1.208786 \\ 9 & \mathrm{C} & 1.210073 & 0.059361 & -0.013542 \\ 10 & \mathrm{H} & -0.550685 & 1.707632 & 0.563856 \\ 11 & \mathrm{O} & 1.453003 & 1.142686 & -0.544700 \\ 12 & \mathrm{C} & 2.188422 & -1.010714 & 0.002972 \\ 13 & \mathrm{H} & 3.181331 & -0.597438 & -0.168067 \\ 14 & \mathrm{H} & 2.164591 & -1.509448 & 0.970763 \\ 15 & \mathrm{H} & 1.954117 & -1.728920 & -0.781376\end{array}$

transition state of C-C cleavage of $c i s-C_{1}$-carboxyl 2-aziridinylmethyl radical

\begin{tabular}{crrrr} 
Center & Atomic & \multicolumn{3}{c}{ Coordinates (Angstroms) } \\
Number & & $\mathrm{X}$ & $\mathrm{Y}$ & $\mathrm{Z}$ \\
- & & & \\
1 & $\mathrm{~N}$ & 1.170698 & -1.195332 & 0.007123 \\
2 & $\mathrm{C}$ & 0.073164 & -0.626172 & -0.719810 \\
3 & $\mathrm{C}$ & 1.628539 & 0.124255 & -0.360917 \\
4 & $\mathrm{C}$ & 1.715236 & 1.216652 & 0.502177 \\
5 & $\mathrm{H}$ & 1.207006 & 1.222186 & 1.459699 \\
6 & $\mathrm{H}$ & 2.242399 & 2.111097 & 0.189423 \\
7 & $\mathrm{H}$ & 2.197402 & 0.140383 & -1.287687 \\
8 & $\mathrm{H}$ & 0.915023 & -1.205360 & 0.999291 \\
9 & $\mathrm{H}$ & -0.006173 & -0.884285 & -1.772566 \\
10 & $\mathrm{C}$ & -1.086411 & -0.120501 & -0.011162 \\
11 & $\mathrm{O}$ & -1.952286 & 0.782254 & -0.639886 \\
12 & $\mathrm{O}$ & -1.307504 & -0.481725 & 1.132969 \\
13 & $\mathrm{H}$ & -2.655392 & 1.013676 & -0.044415
\end{tabular}

transition state of C-C cleavage of trans- $C_{1}$-carboxyl 2-aziridinylmethyl radical

\begin{tabular}{ccccc} 
Center & Atomic & \multicolumn{3}{c}{ Coordinates (Angstroms) } \\
Number & & $\mathrm{X}$ & $\mathrm{Y}$ & $\mathrm{Z}$ \\
$-\mathrm{-}$ & & -1.007348 & 0.861699 & 0.698023 \\
1 & $\mathrm{~N}$ & -0.024695 & -0.171540 & 0.688859 \\
2 & $\mathrm{C}$ & -1.511240 & 0.115357 & -0.430500 \\
3 & $\mathrm{C}$ & -2.650987 & -0.650264 & -0.338204 \\
4 & $\mathrm{C}$ & -3.188663 & -0.730125 & 0.600716 \\
5 & $\mathrm{H}$ & -2.986826 & -1.245308 & -1.180747 \\
6 & $\mathrm{H}$ & -1.039004 & 0.270426 & -1.401937 \\
7 & $\mathrm{H}$ & -0.624042 & 1.762838 & 0.418493 \\
8 & $\mathrm{H}$ & -0.161785 & -0.991722 & 1.383152 \\
9 & $\mathrm{H}$ & & &
\end{tabular}




$\begin{array}{rrrrr}10 & \mathrm{C} & 1.255574 & -0.005693 & 0.028627 \\ 11 & \mathrm{O} & 2.191387 & -1.046849 & 0.044411 \\ 12 & \mathrm{O} & 1.517272 & 1.041139 & -0.540632 \\ 13 & \mathrm{H} & 2.970567 & -0.779487 & -0.428767\end{array}$

transition state of $\mathrm{C}$-C cleavage of $c i s-C_{1}$-acylamino 2-aziridinylmethyl radical

\begin{tabular}{|c|c|c|c|c|}
\hline \multirow{2}{*}{$\begin{array}{l}\text { Center } \\
\text { Number }\end{array}$} & \multirow[t]{2}{*}{ Atomic } & \multicolumn{3}{|c|}{ Coordinates (Angstroms) } \\
\hline & & $\mathrm{X}$ & $\mathrm{Y}$ & $\mathrm{Z}$ \\
\hline 1 & $\mathrm{~N}$ & 1.211718 & -1.161625 & 0.085131 \\
\hline 2 & $\mathrm{C}$ & 0.009536 & -0.816050 & -0.607842 \\
\hline 3 & $\mathrm{C}$ & 1.625227 & 0.124591 & -0.414183 \\
\hline 4 & $\mathrm{C}$ & 1.668777 & 1.288720 & 0.315976 \\
\hline 5 & $\mathrm{H}$ & 2.071358 & 2.197755 & -0.116730 \\
\hline 6 & $\mathrm{H}$ & 1.281313 & 1.342177 & 1.330164 \\
\hline 7 & $\mathrm{H}$ & 2.100533 & 0.075500 & -1.390627 \\
\hline 8 & $\mathrm{H}$ & 1.089100 & -1.130584 & 1.097151 \\
\hline 9 & $\mathrm{C}$ & -1.092380 & -0.164766 & 0.073414 \\
\hline 10 & $\mathrm{H}$ & -0.149001 & -1.250447 & -1.589394 \\
\hline 11 & $\mathrm{O}$ & -1.178057 & -0.223164 & 1.289000 \\
\hline 12 & $\mathrm{~N}$ & -2.085571 & 0.550955 & -0.684818 \\
\hline 13 & $\mathrm{H}$ & -2.453895 & 1.305999 & -0.127764 \\
\hline 14 & $\mathrm{H}$ & -1.664943 & 0.924628 & -1.521182 \\
\hline
\end{tabular}

transition state of C-C cleavage of trans- $C_{l}$-acylamino 2-aziridinylmethyl radical

\begin{tabular}{ccccc} 
Center & Atomic & \multicolumn{3}{c}{ Coordinates (Angstroms) } \\
Number & & $\mathrm{X}$ & $\mathrm{Y}$ & $\mathrm{Z}$ \\
$-\mathrm{-}$ & & -0.845608 & 0.705697 & 0.849359 \\
1 & $\mathrm{~N}$ & -0.025693 & -0.441075 & 0.609508 \\
2 & $\mathrm{C}$ & -1.441199 & 0.283740 & -0.392364 \\
3 & $\mathrm{C}$ & -2.626533 & -0.401458 & -0.517716 \\
4 & $\mathrm{C}$ & -3.044218 & -0.608730 & -1.496607 \\
5 & $\mathrm{H}$ & -3.164295 & -0.776705 & 0.349213 \\
6 & $\mathrm{H}$ & -1.971753 & 0.725486 & -1.267721 \\
7 & $\mathrm{H}$ & -0.273163 & -1.410230 & 1.032283 \\
8 & $\mathrm{H}$ & 1.292405 & -0.220796 & 0.046840 \\
9 & $\mathrm{H}$ & 2.027245 & -1.169069 & -0.174947 \\
10 & $\mathrm{C}$ & 1.734059 & 1.117235 & -0.250167 \\
11 & $\mathrm{O}$ & 2.374627 & 1.094716 & -1.028132 \\
12 & $\mathrm{~N}$ & & &
\end{tabular}


14 $\mathrm{H}$ 0.938303 1.689024

$-0.486584$

transition state of C-C cleavage of $c i s$ - $C_{1}$-vinyl 2-aziridinylmethyl radical

\begin{tabular}{|c|c|c|c|c|}
\hline \multirow{2}{*}{$\begin{array}{l}\text { Center } \\
\text { Number }\end{array}$} & \multirow[t]{2}{*}{ Atomic } & \multicolumn{3}{|c|}{ Coordinates (Angstroms) } \\
\hline & & $\mathrm{X}$ & $\mathrm{Y}$ & $\mathrm{Z}$ \\
\hline 1 & $\mathrm{~N}$ & 0.839047 & -1.123535 & 0.498785 \\
\hline 2 & $\mathrm{C}$ & -0.338625 & -0.740547 & -0.216255 \\
\hline 3 & $\mathrm{C}$ & 1.440360 & -0.157766 & -0.384599 \\
\hline 4 & $\mathrm{C}$ & 1.785447 & 1.129135 & -0.045444 \\
\hline 5 & $\mathrm{H}$ & 2.309707 & 1.764740 & -0.750291 \\
\hline 6 & $\mathrm{H}$ & 1.523810 & 1.555652 & 0.919540 \\
\hline 7 & $\mathrm{H}$ & 1.792463 & -0.588365 & -1.318610 \\
\hline 8 & $\mathrm{H}$ & 0.831138 & -0.775975 & 1.457599 \\
\hline 9 & $\mathrm{C}$ & -1.215593 & 0.302341 & 0.279541 \\
\hline 10 & $\mathrm{H}$ & -0.680198 & -1.394517 & -1.011825 \\
\hline 11 & $\mathrm{C}$ & -2.475694 & 0.377330 & -0.154909 \\
\hline 12 & $\mathrm{H}$ & -0.846323 & 1.024415 & 1.006294 \\
\hline 13 & $\mathrm{H}$ & -3.134327 & 1.160575 & 0.217451 \\
\hline 14 & $\mathrm{H}$ & -2.844964 & -0.344744 & -0.881662 \\
\hline
\end{tabular}

transition state of C-C cleavage of trans- $C_{1}$-vinyl 2-aziridinylmethyl radical

\begin{tabular}{|c|c|c|c|c|}
\hline \multirow{2}{*}{$\begin{array}{l}\text { Center } \\
\text { Number }\end{array}$} & \multirow[t]{2}{*}{ Atomic } & \multicolumn{3}{|c|}{ Coordinates (Angstroms) } \\
\hline & & $\mathrm{X}$ & $\mathrm{Y}$ & $\mathrm{Z}$ \\
\hline 1 & $\mathrm{~N}$ & 0.685873 & 1.134185 & -0.024824 \\
\hline 2 & $\mathrm{C}$ & -0.310571 & 0.196911 & -0.441346 \\
\hline 3 & $\mathrm{C}$ & 1.306762 & -0.063352 & 0.480206 \\
\hline 4 & $\mathrm{C}$ & 2.346298 & -0.736908 & -0.116380 \\
\hline 5 & $\mathrm{H}$ & 2.809005 & -1.585730 & 0.374591 \\
\hline 6 & $\mathrm{H}$ & 2.716809 & -0.460457 & -1.100180 \\
\hline 7 & $\mathrm{H}$ & 1.016472 & -0.302219 & 1.500227 \\
\hline 8 & $\mathrm{H}$ & 1.212777 & 1.504897 & -0.815474 \\
\hline 9 & $\mathrm{H}$ & -0.294104 & -0.239952 & -1.435389 \\
\hline 10 & $\mathrm{C}$ & -1.502225 & 0.073278 & 0.375463 \\
\hline 11 & $\mathrm{C}$ & -2.613307 & -0.456978 & -0.140823 \\
\hline 12 & $\mathrm{H}$ & -1.490855 & 0.412971 & 1.410064 \\
\hline 13 & $\mathrm{H}$ & -3.508280 & -0.549831 & 0.472629 \\
\hline 14 & $\mathrm{H}$ & -2.624677 & -0.796671 & -1.175424 \\
\hline
\end{tabular}


transition state of $\mathrm{C}$-C cleavage of $c i s$ - $C_{1}$-ethynyl 2-aziridinylmethyl radical

\begin{tabular}{|c|c|c|c|c|}
\hline \multirow{2}{*}{$\begin{array}{l}\text { Center } \\
\text { Number }\end{array}$} & \multirow[t]{2}{*}{ Atomic } & \multicolumn{3}{|c|}{ Coordinates (Angstroms) } \\
\hline & & $\mathrm{X}$ & Y & $\mathrm{Z}$ \\
\hline 1 & $\mathrm{~N}$ & 0.821831 & -1.032276 & 0.597474 \\
\hline 2 & $\mathrm{C}$ & -0.246306 & -0.962511 & -0.350730 \\
\hline 3 & $\mathrm{C}$ & 1.370488 & -0.008825 & -0.254687 \\
\hline 4 & $\mathrm{C}$ & 1.367373 & 1.339791 & 0.012614 \\
\hline 5 & $\mathrm{H}$ & 1.875811 & 2.034936 & -0.646107 \\
\hline 6 & $\mathrm{H}$ & 0.838972 & 1.751635 & 0.868618 \\
\hline 7 & $\mathrm{H}$ & 1.980367 & -0.402735 & -1.063792 \\
\hline 8 & $\mathrm{H}$ & 0.554208 & -0.641134 & 1.500563 \\
\hline 9 & $\mathrm{C}$ & -1.400572 & -0.114545 & -0.124625 \\
\hline 10 & $\mathrm{H}$ & -0.281925 & -1.720371 & -1.126397 \\
\hline 11 & $\mathrm{C}$ & -2.347071 & 0.580787 & 0.060781 \\
\hline 12 & $\mathrm{H}$ & -3.183715 & 1.195417 & 0.224669 \\
\hline
\end{tabular}

transition state of C-C cleavage of trans- $C_{1}$-ethynyl 2-aziridinylmethyl radical

\begin{tabular}{|c|c|c|c|c|}
\hline \multirow{2}{*}{$\begin{array}{l}\text { Center } \\
\text { Number }\end{array}$} & \multirow[t]{2}{*}{ Atomic } & \multicolumn{3}{|c|}{ Coordinates (Angstroms) } \\
\hline & & $\mathrm{X}$ & $\mathrm{Y}$ & $\mathrm{Z}$ \\
\hline 1 & $\mathrm{~N}$ & -0.580078 & 1.055149 & -0.336016 \\
\hline 2 & $\mathrm{C}$ & 0.298999 & 0.426278 & 0.594048 \\
\hline 3 & $\mathrm{C}$ & -1.126055 & -0.279123 & -0.414277 \\
\hline 4 & $\mathrm{C}$ & -2.335790 & -0.598342 & 0.158749 \\
\hline 5 & $\mathrm{H}$ & -2.900918 & 0.147081 & 0.708313 \\
\hline 6 & $\mathrm{H}$ & -2.706872 & -1.617398 & 0.134970 \\
\hline 7 & $\mathrm{H}$ & -0.618065 & -1.006268 & -1.049847 \\
\hline 8 & $\mathrm{H}$ & -0.105632 & 1.323853 & -1.196139 \\
\hline 9 & $\mathrm{H}$ & 0.067414 & 0.535983 & 1.646601 \\
\hline 10 & $\mathrm{C}$ & 1.604672 & -0.067114 & 0.201261 \\
\hline 11 & $\mathrm{C}$ & 2.675325 & -0.471695 & -0.120824 \\
\hline 12 & $\mathrm{H}$ & 3.621713 & -0.829319 & -0.405527 \\
\hline
\end{tabular}

transition state of C-C cleavage of $c i s$ - $C_{1}$-phenyl 2-aziridinylmethyl radical

\begin{tabular}{|c|c|c|c|c|}
\hline \multirow{2}{*}{$\begin{array}{l}\text { Center } \\
\text { Number }\end{array}$} & \multirow[t]{2}{*}{ Atomic } & \multicolumn{3}{|c|}{ Coordinates (Angstroms) } \\
\hline & & $X$ & $\mathrm{Y}$ & $\mathrm{Z}$ \\
\hline 1 & $\mathrm{C}$ & 3.617404 & 0.304740 & -0.657874 \\
\hline 2 & $\mathrm{C}$ & 2.184842 & 0.082119 & -0.631312 \\
\hline
\end{tabular}




$\begin{array}{rrrrr}3 & \mathrm{C} & 1.116814 & 0.328118 & 0.796543 \\ 4 & \mathrm{~N} & 1.846505 & -0.820982 & 0.438059 \\ 5 & \mathrm{H} & 1.657959 & 0.879724 & 1.563886 \\ 6 & \mathrm{C} & -0.268046 & 0.168896 & 0.397403 \\ 7 & \mathrm{H} & 2.594645 & -0.837346 & 1.113397 \\ 8 & \mathrm{H} & 1.575351 & 0.110585 & -1.533327 \\ 9 & \mathrm{H} & 3.832021 & 1.223032 & -1.202485 \\ 10 & \mathrm{H} & 4.088526 & -0.267385 & 0.140021 \\ 11 & \mathrm{C} & -1.089568 & 1.293679 & 0.256103 \\ 12 & \mathrm{C} & -2.426673 & 1.139946 & -0.129274 \\ 13 & \mathrm{C} & -2.942257 & -0.138568 & -0.373350 \\ 14 & \mathrm{C} & -2.120735 & -1.263351 & -0.232049 \\ 15 & \mathrm{C} & -0.783630 & -1.109619 & 0.153327 \\ 16 & \mathrm{H} & -0.688517 & 2.288180 & 0.445959 \\ 17 & \mathrm{H} & -3.065700 & 2.014867 & -0.239185 \\ 18 & \mathrm{H} & -3.982334 & -0.258150 & -0.673118 \\ 19 & \mathrm{H} & -2.521786 & -2.257853 & -0.421906 \\ 20 & \mathrm{H} & -0.144603 & -1.984539 & 0.263239\end{array}$

transition state of C-C cleavage of trans- $C_{1}$-phenyl 2-aziridinylmethyl radical

\begin{tabular}{ccccc} 
Center & Atomic & \multicolumn{3}{c}{ Coordinates (Angstroms) } \\
Number & & $\mathrm{X}$ & $\mathrm{Y}$ & $\mathrm{Z}$ \\
\hline 1 & $\mathrm{~N}$ & 2.162876 & -0.340886 & -0.897612 \\
2 & $\mathrm{C}$ & 1.203944 & 0.711806 & -0.823032 \\
3 & $\mathrm{C}$ & 2.504993 & 0.149669 & 0.416469 \\
4 & $\mathrm{C}$ & 2.172638 & -0.547042 & 1.555703 \\
5 & $\mathrm{H}$ & 1.619440 & -1.478183 & 1.492522 \\
6 & $\mathrm{H}$ & 2.391682 & -0.139121 & 2.536704 \\
7 & $\mathrm{H}$ & 3.148615 & 1.028103 & 0.486088 \\
8 & $\mathrm{H}$ & 2.895548 & -0.149479 & -1.578544 \\
9 & $\mathrm{C}$ & -0.147648 & 0.351677 & -0.440924 \\
10 & $\mathrm{H}$ & 1.393205 & 1.693825 & -1.252499 \\
11 & $\mathrm{C}$ & -1.018840 & 1.329001 & 0.054922 \\
12 & $\mathrm{C}$ & -2.323826 & 0.981291 & 0.423853 \\
13 & $\mathrm{C}$ & -2.757620 & -0.343744 & 0.296938 \\
14 & $\mathrm{C}$ & -1.886428 & -1.321067 & -0.198908 \\
15 & $\mathrm{C}$ & -0.581442 & -0.973357 & -0.567840 \\
16 & $\mathrm{H}$ & -0.681410 & 2.359688 & 0.153643 \\
17 & $\mathrm{H}$ & -3.001488 & 1.741509 & 0.809550 \\
18 & $\mathrm{H}$ & -3.772712 & -0.614213 & 0.583913 \\
19 & $\mathrm{H}$ & -2.223858 & -2.351755 & -0.297630 \\
20 & $\mathrm{H}$ & 0.096220 & -1.733575 & -0.953537 \\
& & & &
\end{tabular}


cis- $C_{1}$-methyl trans- $N$-methyl 2-aziridinylmethyl radical

\begin{tabular}{crrrr} 
Center & Atomic & \multicolumn{3}{c}{ Coordinates (Angstroms) } \\
Number & Number & X & Y & Z \\
-1 & N & 0.000000 & 0.000000 & 0.000000 \\
2 & $\mathrm{C}$ & 0.000000 & 0.000000 & 1.448654 \\
3 & $\mathrm{C}$ & 1.316452 & 0.000000 & 0.665103 \\
4 & $\mathrm{C}$ & 2.187813 & -1.153728 & 0.497518 \\
5 & $\mathrm{H}$ & 1.789400 & -2.102893 & 0.159193 \\
6 & $\mathrm{H}$ & 3.231087 & -1.099806 & 0.789398 \\
7 & $\mathrm{H}$ & 1.827924 & 0.965885 & 0.714043 \\
8 & $\mathrm{H}$ & -0.218462 & 0.958312 & 1.931809 \\
9 & $\mathrm{C}$ & -0.543625 & -1.208266 & 2.172349 \\
10 & $\mathrm{C}$ & -0.388361 & 1.235406 & -0.666545 \\
11 & $\mathrm{H}$ & -0.067204 & 2.146200 & -0.129129 \\
12 & $\mathrm{H}$ & 0.048066 & 1.257181 & -1.670944 \\
13 & $\mathrm{H}$ & -1.479776 & 1.263758 & -0.764292 \\
14 & $\mathrm{H}$ & -0.268021 & -2.132078 & 1.657280 \\
15 & $\mathrm{H}$ & -0.155861 & -1.253758 & 3.197025 \\
16 & $\mathrm{H}$ & -1.638479 & -1.164096 & 2.226964
\end{tabular}

trans- $C_{1}$-methyl cis- $N$-methyl 2-aziridinylmethyl radical

\begin{tabular}{|c|c|c|c|c|}
\hline \multirow{2}{*}{$\begin{array}{l}\text { Center } \\
\text { Number }\end{array}$} & \multirow{2}{*}{$\begin{array}{l}\text { Atomic } \\
\text { Number }\end{array}$} & \multicolumn{3}{|c|}{ Coordinates (Angstroms) } \\
\hline & & $\mathrm{X}$ & $\mathrm{Y}$ & $\mathrm{Z}$ \\
\hline 1 & $\mathrm{~N}$ & 0.000000 & 0.000000 & 0.000000 \\
\hline 2 & $\mathrm{C}$ & 0.000000 & 0.000000 & 1.447473 \\
\hline 3 & $\mathrm{C}$ & 1.326518 & 0.000000 & 0.707109 \\
\hline 4 & $\mathrm{C}$ & 2.225128 & -1.138544 & 0.723892 \\
\hline 5 & $\mathrm{H}$ & 3.296450 & -0.985144 & 0.666663 \\
\hline 6 & $\mathrm{H}$ & 1.863968 & -2.159169 & 0.775861 \\
\hline 7 & $\mathrm{H}$ & 1.815243 & 0.969783 & 0.619992 \\
\hline 8 & $\mathrm{H}$ & -0.245059 & -0.951085 & 1.930784 \\
\hline 9 & $\mathrm{C}$ & -0.514207 & 1.229163 & 2.161336 \\
\hline 10 & $\mathrm{C}$ & -0.435023 & -1.215226 & -0.679512 \\
\hline 11 & $\mathrm{H}$ & 0.093146 & -1.306182 & -1.634428 \\
\hline 12 & $\mathrm{H}$ & -0.281090 & -2.144687 & -0.108524 \\
\hline 13 & $\mathrm{H}$ & -1.507166 & -1.122809 & -0.886689 \\
\hline 14 & $\mathrm{H}$ & -0.213052 & 2.136335 & 1.628257 \\
\hline 15 & $\mathrm{H}$ & -0.124651 & 1.278259 & 3.185050 \\
\hline 16 & $\mathrm{H}$ & -1.610159 & 1.218476 & 2.214867 \\
\hline
\end{tabular}


cis- $C_{1}$-formyl trans- $N$-methyl 2-aziridinylmethyl radical

\begin{tabular}{|c|c|c|c|c|}
\hline \multirow{2}{*}{$\begin{array}{l}\text { Center } \\
\text { Number }\end{array}$} & \multirow{2}{*}{$\begin{array}{l}\text { Atomic } \\
\text { Number }\end{array}$} & \multicolumn{3}{|c|}{ Coordinates (Angstroms) } \\
\hline & & $X$ & $\mathrm{Y}$ & $\mathrm{Z}$ \\
\hline 1 & $\mathrm{~N}$ & 0.000000 & 0.000000 & 0.000000 \\
\hline 2 & $\mathrm{C}$ & 0.000000 & 0.000000 & 1.439745 \\
\hline 3 & $\mathrm{C}$ & 1.325313 & 0.000000 & 0.645625 \\
\hline 4 & $\mathrm{C}$ & 2.192304 & -1.156677 & 0.479894 \\
\hline 5 & $\mathrm{H}$ & 1.788034 & -2.117034 & 0.183214 \\
\hline 6 & $\mathrm{H}$ & 3.246212 & -1.085199 & 0.723716 \\
\hline 7 & $\mathrm{H}$ & 1.833661 & 0.965677 & 0.674354 \\
\hline 8 & $\mathrm{H}$ & -0.246989 & 0.958761 & 1.899548 \\
\hline 9 & $\mathrm{C}$ & -0.523193 & -1.212510 & 2.169131 \\
\hline 10 & $\mathrm{C}$ & -0.482762 & 1.059031 & -0.759329 \\
\hline 11 & $\mathrm{O}$ & -1.475281 & 1.704857 & -0.494448 \\
\hline 12 & $\mathrm{H}$ & 0.111655 & 1.213516 & -1.679556 \\
\hline 13 & $\mathrm{H}$ & -0.244749 & -2.137818 & 1.658105 \\
\hline 14 & $\mathrm{H}$ & -1.616663 & -1.170773 & 2.228350 \\
\hline 15 & $\mathrm{H}$ & -0.127569 & -1.245324 & 3.190545 \\
\hline
\end{tabular}

trans- $C_{1}$-formyl cis- $N$-methyl 2-aziridinylmethyl radical

\begin{tabular}{ccccc} 
Center & Atomic & \multicolumn{3}{c}{ Coordinates (Angstroms) } \\
Number & Number & X & Y & Z \\
-1 & N & 0.000000 & 0.000000 & 0.000000 \\
2 & $\mathrm{C}$ & 0.000000 & 0.000000 & 1.438228 \\
3 & $\mathrm{C}$ & 1.327152 & 0.000000 & 0.666705 \\
4 & $\mathrm{C}$ & 2.181279 & -1.174675 & 0.631012 \\
5 & $\mathrm{H}$ & 3.259325 & -1.071494 & 0.611608 \\
6 & $\mathrm{H}$ & 1.756232 & -2.173060 & 0.656027 \\
7 & $\mathrm{H}$ & 1.832762 & 0.962053 & 0.598047 \\
8 & $\mathrm{H}$ & -0.264195 & -0.952302 & 1.900492 \\
9 & $\mathrm{C}$ & -0.495980 & 1.232750 & 2.155570 \\
10 & $\mathrm{C}$ & -0.504853 & -1.036196 & -0.773379 \\
11 & $\mathrm{H}$ & 0.091303 & -1.197566 & -1.690514 \\
12 & $\mathrm{O}$ & -1.523601 & -1.649236 & -0.527223 \\
13 & $\mathrm{H}$ & -0.186563 & 2.141863 & 1.630145 \\
14 & $\mathrm{H}$ & -0.105597 & 1.267460 & 3.178842 \\
15 & $\mathrm{H}$ & -1.590887 & 1.226561 & 2.207096
\end{tabular}

cis- $C_{1}$-phenyl trans- $N$-methyl 2-aziridinylmethyl radical

Center Atomic Coordinates (Angstroms)




$\begin{array}{crrrr}\text { Number } & \text { Number } & \text { X } & \text { Y } & \text { Z } \\ -1 & \mathrm{~N} & 0.000000 & 0.000000 & 0.000000 \\ 2 & \mathrm{C} & 0.000000 & 0.000000 & 1.447222 \\ 3 & \mathrm{C} & 1.317552 & 0.000000 & 0.658947 \\ 4 & \mathrm{C} & 2.187924 & -1.154339 & 0.491250 \\ 5 & \mathrm{H} & 1.790166 & -2.104106 & 0.153895 \\ 6 & \mathrm{H} & 3.230325 & -1.098476 & 0.785139 \\ 7 & \mathrm{H} & 1.823496 & 0.967632 & 0.697233 \\ 8 & \mathrm{H} & -0.222596 & 0.959810 & 1.922000 \\ 9 & \mathrm{C} & -0.536505 & -1.209667 & 2.173121 \\ 10 & \mathrm{C} & -0.431781 & 1.126062 & -0.734388 \\ 11 & \mathrm{C} & 0.348205 & 1.620609 & -1.791137 \\ 12 & \mathrm{C} & -0.110036 & 2.688286 & -2.560358 \\ 13 & \mathrm{C} & -1.348605 & 3.277110 & -2.293472 \\ 14 & \mathrm{C} & -2.127368 & 2.782946 & -1.245160 \\ 15 & \mathrm{C} & -1.676501 & 1.716526 & -0.468322 \\ 16 & \mathrm{H} & -2.288619 & 1.329106 & 0.341673 \\ 17 & \mathrm{H} & -3.093828 & 3.230428 & -1.026930 \\ 18 & \mathrm{H} & -1.702099 & 4.109551 & -2.895194 \\ 19 & \mathrm{H} & 0.506392 & 3.062728 & -3.373776 \\ 20 & \mathrm{H} & 1.308310 & 1.157978 & -2.002397 \\ 21 & \mathrm{H} & -0.265392 & -2.133417 & 1.655564 \\ 22 & \mathrm{H} & -0.138794 & -1.253461 & 3.193781 \\ 23 & \mathrm{H} & -1.630569 & -1.165966 & 2.238513\end{array}$

trans- $C_{1}$-phenyl cis- $N$-methyl 2-aziridinylmethyl radical

\begin{tabular}{ccrrr} 
Center & Atomic & \multicolumn{3}{c}{ Coordinates (Angstroms) } \\
Number & Number & X & Y & Z \\
-1 & N & 0.000000 & 0.000000 & 0.000000 \\
2 & $\mathrm{C}$ & 0.000000 & 0.000000 & 1.444317 \\
3 & $\mathrm{C}$ & 1.327861 & 0.000000 & 0.701050 \\
4 & $\mathrm{C}$ & 2.199813 & -1.158548 & 0.709069 \\
5 & $\mathrm{H}$ & 1.797915 & -2.165146 & 0.695475 \\
6 & $\mathrm{H}$ & 3.276041 & -1.032967 & 0.697300 \\
7 & $\mathrm{H}$ & 1.824903 & 0.965632 & 0.619720 \\
8 & $\mathrm{H}$ & -0.241377 & -0.951632 & 1.924856 \\
9 & $\mathrm{C}$ & -0.511665 & 1.230513 & 2.157143 \\
10 & $\mathrm{C}$ & -0.467528 & -1.105913 & -0.743337 \\
11 & $\mathrm{C}$ & -1.659312 & -1.757932 & -0.393351 \\
12 & $\mathrm{C}$ & -2.162889 & -2.783232 & -1.194974 \\
13 & $\mathrm{C}$ & -1.485076 & -3.179582 & -2.348173 \\
14 & $\mathrm{C}$ & -0.296976 & -2.530842 & -2.698413
\end{tabular}




$\begin{array}{llrrr}15 & \mathrm{C} & 0.208034 & -1.500284 & -1.910215 \\ 16 & \mathrm{H} & 1.127592 & -0.992427 & -2.185298 \\ 17 & \mathrm{H} & 0.240638 & -2.828471 & -3.595252 \\ 18 & \mathrm{H} & -1.876614 & -3.981162 & -2.968032 \\ 19 & \mathrm{H} & -3.090478 & -3.274372 & -0.911608 \\ 20 & \mathrm{H} & -2.197020 & -1.448387 & 0.498656 \\ 21 & \mathrm{H} & -1.606867 & 1.217889 & 2.218449 \\ 22 & \mathrm{H} & -0.114846 & 1.280194 & 3.177756 \\ 23 & \mathrm{H} & -0.216089 & 2.137575 & 1.620731\end{array}$

cis- $C_{1}$-methyl trans- $N$-formyl 2-aziridinylmethyl radical

\begin{tabular}{ccrrr} 
Center & Atomic & \multicolumn{3}{c}{ Coordinates (Angstroms) } \\
Number & Number & X & Y & Z \\
-1 & N & 0.000000 & 0.000000 & 0.000000 \\
2 & $\mathrm{C}$ & 0.000000 & 0.000000 & 1.451942 \\
3 & $\mathrm{C}$ & 1.317406 & 0.000000 & 0.604992 \\
4 & $\mathrm{C}$ & 2.192752 & -1.143459 & 0.421546 \\
5 & $\mathrm{H}$ & 1.806114 & -2.074800 & 0.024794 \\
6 & $\mathrm{H}$ & 3.220329 & -1.103025 & 0.764773 \\
7 & $\mathrm{H}$ & 1.816645 & 0.969385 & 0.700465 \\
8 & $\mathrm{H}$ & -0.196734 & 0.932200 & 1.985367 \\
9 & $\mathrm{C}$ & -0.467405 & -1.226903 & 2.144605 \\
10 & $\mathrm{C}$ & -0.441785 & 1.227172 & -0.653330 \\
11 & $\mathrm{H}$ & -0.100653 & 2.142237 & -0.139096 \\
12 & $\mathrm{H}$ & -1.535856 & 1.236727 & -0.689942 \\
13 & $\mathrm{H}$ & -0.057747 & 1.239874 & -1.678173 \\
14 & $\mathrm{H}$ & -0.445225 & -2.148376 & 1.524976 \\
15 & $\mathrm{O}$ & -0.839937 & -1.239050 & 3.299843
\end{tabular}

trans- $C_{1}$-methyl cis- $N$-formyl 2-aziridinylmethyl radical

\begin{tabular}{|c|c|c|c|c|}
\hline \multirow{2}{*}{$\begin{array}{l}\text { Center } \\
\text { Number }\end{array}$} & \multirow{2}{*}{$\begin{array}{l}\text { Atomic } \\
\text { Number }\end{array}$} & \multicolumn{3}{|c|}{ Coordinates (Angstroms) } \\
\hline & & $X$ & Y & $\mathrm{Z}$ \\
\hline 1 & $\mathrm{~N}$ & 0.000000 & 0.000000 & 0.000000 \\
\hline 2 & $\mathrm{C}$ & 0.000000 & 0.000000 & 1.448236 \\
\hline 3 & $\mathrm{C}$ & 1.326546 & 0.000000 & 0.619340 \\
\hline 4 & $\mathrm{C}$ & 2.213809 & -1.139764 & 0.666094 \\
\hline 5 & $\mathrm{H}$ & 1.851840 & -2.159155 & 0.608528 \\
\hline 6 & $\mathrm{H}$ & 3.276216 & -0.984423 & 0.810388 \\
\hline 7 & $\mathrm{H}$ & 1.817004 & 0.970090 & 0.545212 \\
\hline 8 & $\mathrm{H}$ & -0.211545 & -0.927110 & 1.982736 \\
\hline 9 & $\mathrm{C}$ & -0.399161 & 1.248862 & 2.143804 \\
\hline
\end{tabular}




$\begin{array}{lllll}10 & \mathrm{C} & -0.491088 & -1.201855 & -0.668430 \\ 11 & \mathrm{H} & -0.314235 & -2.137378 & -0.116304 \\ 12 & \mathrm{H} & -1.570551 & -1.091555 & -0.811262 \\ 13 & \mathrm{H} & -0.015010 & -1.279698 & -1.650696 \\ 14 & \mathrm{H} & -0.357916 & 2.158774 & 1.505440 \\ 15 & \mathrm{O} & -0.719071 & 1.296264 & 3.314294\end{array}$

cis- $C_{1}$-formyl trans- $N$-formyl 2-aziridinylmethyl radical

\begin{tabular}{crrrr} 
Center & Atomic & \multicolumn{3}{c}{ Coordinates (Angstroms) } \\
Number & Number & X & Y & Z \\
-1 & $\mathrm{~N}$ & 0.000000 & 0.000000 & 0.000000 \\
2 & $\mathrm{C}$ & 0.000000 & 0.000000 & 1.438888 \\
3 & $\mathrm{C}$ & 1.327640 & 0.000000 & 0.591881 \\
4 & $\mathrm{C}$ & 2.198771 & -1.146782 & 0.406453 \\
5 & $\mathrm{H}$ & 1.806191 & -2.088498 & 0.041656 \\
6 & $\mathrm{H}$ & 3.238294 & -1.089555 & 0.707350 \\
7 & $\mathrm{H}$ & 1.824447 & 0.969306 & 0.665403 \\
8 & $\mathrm{H}$ & -0.237084 & 0.930865 & 1.952309 \\
9 & $\mathrm{C}$ & -0.438742 & -1.242966 & 2.137183 \\
10 & $\mathrm{C}$ & -0.530983 & 1.052811 & -0.749716 \\
11 & $\mathrm{O}$ & -1.519770 & 1.680041 & -0.446029 \\
12 & $\mathrm{H}$ & 0.028723 & 1.207331 & -1.689983 \\
13 & $\mathrm{H}$ & -0.420444 & -2.163404 & 1.516119 \\
14 & $\mathrm{O}$ & -0.780593 & -1.256747 & 3.298745
\end{tabular}

trans- $C_{1}$-formyl cis- $N$-formyl 2-aziridinylmethyl radical

\begin{tabular}{crrrr} 
Center & Atomic & \multicolumn{3}{c}{ Coordinates (Angstroms) } \\
Number & Number & X & Y & Z \\
- & Nume & & \\
1 & $\mathrm{~N}$ & 0.000000 & 0.000000 & 0.000000 \\
2 & $\mathrm{C}$ & 0.000000 & 0.000000 & 1.436118 \\
3 & $\mathrm{C}$ & 1.329832 & 0.000000 & 0.598321 \\
4 & $\mathrm{C}$ & 2.170556 & -1.178397 & 0.596359 \\
5 & $\mathrm{H}$ & 1.737090 & -2.172939 & 0.575848 \\
6 & $\mathrm{H}$ & 3.243975 & -1.080202 & 0.700993 \\
7 & $\mathrm{H}$ & 1.838943 & 0.960267 & 0.530439 \\
8 & $\mathrm{H}$ & -0.245625 & -0.925276 & 1.953613 \\
9 & $\mathrm{C}$ & -0.383365 & 1.263644 & 2.128527 \\
10 & $\mathrm{C}$ & -0.546811 & -1.027688 & -0.769131 \\
11 & $\mathrm{O}$ & -1.560983 & -1.626800 & -0.490885 \\
12 & $\mathrm{H}$ & 0.021610 & -1.183828 & -1.703477 \\
13 & $\mathrm{H}$ & -0.342190 & 2.170762 & 1.486580
\end{tabular}


cis- $C_{1}$-phenyl trans- $N$-formyl 2-aziridinylmethyl radical

\begin{tabular}{|c|c|c|c|c|}
\hline \multirow{3}{*}{$\begin{array}{l}\text { Center } \\
\text { Number } \\
\text {------ } \\
1\end{array}$} & \multirow{3}{*}{$\begin{array}{c}\text { Atomic } \\
\text { Number } \\
\text { N }\end{array}$} & \multicolumn{3}{|c|}{ Coordinates (Angstroms) } \\
\hline & & $\mathrm{X}$ & $\mathrm{Y}$ & $\mathrm{Z}$ \\
\hline & & 0.000000 & 0.000000 & 0.000000 \\
\hline 2 & $\mathrm{C}$ & 0.000000 & 0.000000 & 1.448633 \\
\hline 3 & $\mathrm{C}$ & 1.319743 & 0.000000 & 0.600274 \\
\hline 4 & $\mathrm{C}$ & 2.192982 & -1.144367 & 0.414988 \\
\hline 5 & $\mathrm{H}$ & 1.805844 & -2.075727 & 0.018809 \\
\hline 6 & $\mathrm{H}$ & 3.220353 & -1.103595 & 0.758437 \\
\hline 7 & $\mathrm{H}$ & 1.813855 & 0.971491 & 0.683693 \\
\hline 8 & $\mathrm{H}$ & -0.201814 & 0.933585 & 1.975534 \\
\hline 9 & $\mathrm{C}$ & -0.460363 & -1.231888 & 2.142181 \\
\hline 10 & $\mathrm{C}$ & -0.475975 & 1.126570 & -0.716751 \\
\hline 11 & $\mathrm{C}$ & -1.730329 & 1.677205 & -0.421932 \\
\hline 12 & $\mathrm{C}$ & -2.219493 & 2.739834 & -1.180453 \\
\hline 13 & $\mathrm{C}$ & -1.468805 & 3.263997 & -2.234757 \\
\hline 14 & $\mathrm{C}$ & -0.219980 & 2.711690 & -2.527918 \\
\hline 15 & $\mathrm{C}$ & 0.277987 & 1.648202 & -1.776989 \\
\hline 16 & $\mathrm{H}$ & 1.245533 & 1.211983 & -2.009142 \\
\hline 17 & $\mathrm{H}$ & 0.373582 & 3.110375 & -3.346420 \\
\hline 18 & $\mathrm{H}$ & -1.852611 & 4.093474 & -2.821543 \\
\hline 19 & $\mathrm{H}$ & -3.193053 & 3.160252 & -0.942730 \\
\hline 20 & $\mathrm{H}$ & -2.317875 & 1.266863 & 0.394702 \\
\hline 21 & $\mathrm{H}$ & -0.453482 & -2.149637 & 1.516705 \\
\hline 22 & $\mathrm{O}$ & -0.807715 & -1.247683 & 3.304479 \\
\hline
\end{tabular}

trans- $C_{1}$-phenyl cis- $N$-formyl 2-aziridinylmethyl radical

\begin{tabular}{ccccc} 
Center & Atomic & \multicolumn{3}{c}{ Coordinates (Angstroms) } \\
Number & Number & X & Y & Z \\
------------1000 & \\
1 & N & 0.000000 & 0.000000 & 0.000000 \\
2 & $\mathrm{C}$ & 0.000000 & 0.000000 & 1.443318 \\
3 & $\mathrm{C}$ & 1.327798 & 0.000000 & 0.621025 \\
4 & $\mathrm{C}$ & 2.187651 & -1.161458 & 0.662701 \\
5 & $\mathrm{H}$ & 1.783800 & -2.163780 & 0.582927 \\
6 & $\mathrm{H}$ & 3.253523 & -1.038733 & 0.812173 \\
7 & $\mathrm{H}$ & 1.828153 & 0.964533 & 0.546535 \\
8 & $\mathrm{H}$ & -0.211113 & -0.926005 & 1.978077 \\
9 & $\mathrm{C}$ & -0.406226 & 1.254052 & 2.132425 \\
10 & $\mathrm{C}$ & -0.514716 & -1.097644 & -0.734955
\end{tabular}




$\begin{array}{llrrr}11 & \mathrm{C} & -1.708846 & -1.721221 & -0.351025 \\ 12 & \mathrm{C} & -2.253920 & -2.733285 & -1.142502 \\ 13 & \mathrm{C} & -1.615501 & -3.137821 & -2.315035 \\ 14 & \mathrm{C} & -0.424359 & -2.513607 & -2.696602 \\ 15 & \mathrm{C} & 0.123954 & -1.497136 & -1.918595 \\ 16 & \mathrm{H} & 1.045206 & -1.006387 & -2.218095 \\ 17 & \mathrm{H} & 0.081679 & -2.818040 & -3.609028 \\ 18 & \mathrm{H} & -2.040253 & -3.928663 & -2.926469 \\ 19 & \mathrm{H} & -3.182789 & -3.206686 & -0.835549 \\ 20 & \mathrm{H} & -2.214515 & -1.404176 & 0.556688 \\ 21 & \mathrm{H} & -0.375404 & 2.159732 & 1.487754 \\ 22 & \mathrm{O} & -0.718925 & 1.304430 & 3.303624\end{array}$

cis- $C_{1}$-methyl trans- $N$-phenyl 2-aziridinylmethyl radical

\begin{tabular}{|c|c|c|c|c|}
\hline \multirow{2}{*}{$\begin{array}{l}\text { Center } \\
\text { Number }\end{array}$} & \multirow{2}{*}{$\begin{array}{l}\text { Atomic } \\
\text { Number }\end{array}$} & \multicolumn{3}{|c|}{ Coordinates (Angstroms) } \\
\hline & & $\mathrm{X}$ & $\mathrm{Y}$ & $\mathrm{Z}$ \\
\hline 1 & $\mathrm{~N}$ & 0.000000 & 0.000000 & 0.000000 \\
\hline 2 & $\mathrm{C}$ & 0.000000 & 0.000000 & 1.443932 \\
\hline 3 & $\mathrm{C}$ & 1.325348 & 0.000000 & 0.629803 \\
\hline 4 & $\mathrm{C}$ & 2.196668 & -1.147849 & 0.471240 \\
\hline 5 & $\mathrm{H}$ & 1.787501 & -2.127483 & 0.256142 \\
\hline 6 & $\mathrm{H}$ & 3.251178 & -1.065414 & 0.710419 \\
\hline 7 & $\mathrm{H}$ & 1.828212 & 0.969864 & 0.682958 \\
\hline 8 & $\mathrm{H}$ & -0.179333 & 0.960946 & 1.936523 \\
\hline 9 & $\mathrm{C}$ & -0.530281 & -1.176686 & 2.186721 \\
\hline 10 & $\mathrm{C}$ & -0.413795 & 1.227724 & -0.665467 \\
\hline 11 & $\mathrm{H}$ & -1.506987 & 1.247432 & -0.733679 \\
\hline 12 & $\mathrm{H}$ & -0.001446 & 1.243670 & -1.679772 \\
\hline 13 & $\mathrm{H}$ & -0.082804 & 2.141412 & -0.140230 \\
\hline 14 & $\mathrm{C}$ & -0.983826 & -2.324709 & 1.523255 \\
\hline 15 & $\mathrm{C}$ & -1.466146 & -3.415351 & 2.247013 \\
\hline 16 & $\mathrm{C}$ & -1.502989 & -3.376083 & 3.642386 \\
\hline 17 & $\mathrm{C}$ & -1.057481 & -2.233777 & 4.311250 \\
\hline 18 & $\mathrm{C}$ & -0.576809 & -1.143112 & 3.587796 \\
\hline 19 & $\mathrm{H}$ & -0.232796 & -0.254460 & 4.113267 \\
\hline 20 & $\mathrm{H}$ & -1.087039 & -2.190733 & 5.397029 \\
\hline 21 & $\mathrm{H}$ & -1.879762 & -4.226526 & 4.204484 \\
\hline 22 & $\mathrm{H}$ & -1.819382 & -4.297449 & 1.718681 \\
\hline 23 & $\mathrm{H}$ & -0.964973 & -2.340372 & 0.438117 \\
\hline
\end{tabular}

trans- $C_{1}$-methyl $c i s$ - $N$-phenyl 2-aziridinylmethyl radical

Center Atomic Coordinates (Angstroms)




$\begin{array}{rrrrr}\text { Number } & \text { Number } & \text { X } & \text { Y } & \text { Z } \\ -1 & \text { N } & 0.000000 & 0.000000 & 0.000000 \\ 2 & \mathrm{C} & 0.000000 & 0.000000 & 1.444557 \\ 3 & \mathrm{C} & 1.330412 & 0.000000 & 0.642888 \\ 4 & \mathrm{C} & 2.227469 & -1.133794 & 0.649568 \\ 5 & \mathrm{H} & 3.293291 & -0.975482 & 0.764033 \\ 6 & \mathrm{H} & 1.870783 & -2.154874 & 0.586358 \\ 7 & \mathrm{H} & 1.808800 & 0.976921 & 0.583713 \\ 8 & \mathrm{H} & -0.202778 & -0.954895 & 1.936553 \\ 9 & \mathrm{C} & -0.470332 & 1.199899 & 2.186959 \\ 10 & \mathrm{C} & -0.469648 & -1.202414 & -0.678618 \\ 11 & \mathrm{H} & -0.310616 & -2.136921 & -0.117171 \\ 12 & \mathrm{H} & 0.036005 & -1.289166 & -1.645778 \\ 13 & \mathrm{H} & -1.544955 & -1.095523 & -0.855846 \\ 14 & \mathrm{C} & -0.745635 & 2.404896 & 1.524722 \\ 15 & \mathrm{C} & -1.157924 & 3.526438 & 2.243293 \\ 16 & \mathrm{C} & -1.301189 & 3.462347 & 3.631405 \\ 17 & \mathrm{C} & -1.032454 & 2.264596 & 4.297640 \\ 18 & \mathrm{C} & -0.620900 & 1.142249 & 3.580136 \\ 19 & \mathrm{H} & -0.413738 & 0.210720 & 4.102954 \\ 20 & \mathrm{H} & -1.146631 & 2.203155 & 5.376907 \\ 21 & \mathrm{H} & -1.623929 & 4.337340 & 4.189381 \\ 22 & \mathrm{H} & -1.373790 & 4.452965 & 1.717089 \\ 23 & \mathrm{H} & -0.647161 & 2.439308 & 0.443871\end{array}$

cis- $C_{1}$-formyl trans- $N$-phenyl 2-aziridinylmethyl radical

\begin{tabular}{ccccc} 
Center & Atomic & \multicolumn{3}{c}{ Coordinates (Angstroms) } \\
Number & Number & X & Y & Z \\
- & & & & \\
1 & $\mathrm{~N}$ & 0.000000 & 0.000000 & 0.000000 \\
2 & $\mathrm{C}$ & 0.000000 & 0.000000 & 1.435612 \\
3 & $\mathrm{C}$ & 1.335429 & 0.000000 & 0.615076 \\
4 & $\mathrm{C}$ & 2.200014 & -1.152991 & 0.459938 \\
5 & $\mathrm{H}$ & 1.785123 & -2.138654 & 0.288611 \\
6 & $\mathrm{H}$ & 3.263603 & -1.058469 & 0.646339 \\
7 & $\mathrm{H}$ & 1.835943 & 0.969148 & 0.644493 \\
8 & $\mathrm{H}$ & -0.216759 & 0.960352 & 1.906086 \\
9 & $\mathrm{C}$ & -0.495460 & -1.189682 & 2.182572 \\
10 & $\mathrm{C}$ & -0.492012 & 1.061549 & -0.754956 \\
11 & $\mathrm{O}$ & -1.475888 & 1.712218 & -0.476279 \\
12 & $\mathrm{H}$ & 0.093696 & 1.211937 & -1.681104 \\
13 & $\mathrm{C}$ & -0.949788 & -2.340898 & 1.526733 \\
14 & $\mathrm{C}$ & -1.388141 & -3.443583 & 2.260317
\end{tabular}




$\begin{array}{lllll}15 & \mathrm{C} & -1.380067 & -3.409596 & 3.655990 \\ 16 & \mathrm{C} & -0.935811 & -2.262060 & 4.316588 \\ 17 & \mathrm{C} & -0.498593 & -1.159697 & 3.583993 \\ 18 & \mathrm{H} & -0.157070 & -0.266008 & 4.101858 \\ 19 & \mathrm{H} & -0.933454 & -2.223682 & 5.402652 \\ 20 & \mathrm{H} & -1.724237 & -4.268553 & 4.225673 \\ 21 & \mathrm{H} & -1.743871 & -4.328751 & 1.739529 \\ 22 & \mathrm{H} & -0.971957 & -2.356287 & 0.441383\end{array}$

trans- $C_{1}$-formyl $c i s-N$-phenyl 2-aziridinylmethyl radical

\begin{tabular}{|c|c|c|c|c|}
\hline \multirow{2}{*}{$\begin{array}{l}\text { Center } \\
\text { Number }\end{array}$} & \multirow{2}{*}{$\begin{array}{l}\text { Atomic } \\
\text { Number }\end{array}$} & \multicolumn{3}{|c|}{ Coordinates (Angstroms) } \\
\hline & & $X$ & $\mathrm{Y}$ & $\mathrm{Z}$ \\
\hline 1 & $\mathrm{~N}$ & 0.000000 & 0.000000 & 0.000000 \\
\hline 2 & $\mathrm{C}$ & 0.000000 & 0.000000 & 1.435145 \\
\hline 3 & $\mathrm{C}$ & 1.334395 & 0.000000 & 0.613104 \\
\hline 4 & $\mathrm{C}$ & 2.185739 & -1.169624 & 0.571808 \\
\hline 5 & $\mathrm{H}$ & 1.761994 & -2.168220 & 0.535448 \\
\hline 6 & $\mathrm{H}$ & 3.259606 & -1.066472 & 0.668832 \\
\hline 7 & $\mathrm{H}$ & 1.829377 & 0.968338 & 0.564793 \\
\hline 8 & $\mathrm{H}$ & -0.230692 & -0.954882 & 1.907206 \\
\hline 9 & $\mathrm{C}$ & -0.442372 & 1.209130 & 2.180073 \\
\hline 10 & $\mathrm{C}$ & -0.522101 & -1.029045 & -0.775732 \\
\hline 11 & $\mathrm{O}$ & -1.541086 & -1.636470 & -0.524660 \\
\hline 12 & $\mathrm{H}$ & 0.070897 & -1.189024 & -1.695089 \\
\hline 13 & $\mathrm{C}$ & -0.702333 & 2.420948 & 1.525560 \\
\hline 14 & $\mathrm{C}$ & -1.079467 & 3.547833 & 2.254873 \\
\hline 15 & $\mathrm{C}$ & -1.202819 & 3.479377 & 3.644456 \\
\hline 16 & $\mathrm{C}$ & -0.950836 & 2.273488 & 4.302192 \\
\hline 17 & $\mathrm{C}$ & -0.573617 & 1.145967 & 3.574438 \\
\hline 18 & $\mathrm{H}$ & -0.381770 & 0.207186 & 4.089341 \\
\hline 19 & $\mathrm{H}$ & -1.052139 & 2.209268 & 5.382285 \\
\hline 20 & $\mathrm{H}$ & -1.499763 & 4.358076 & 4.210514 \\
\hline 21 & $\mathrm{H}$ & -1.284994 & 4.480286 & 1.735715 \\
\hline 22 & $\mathrm{H}$ & -0.624302 & 2.464904 & 0.443141 \\
\hline
\end{tabular}

cis- $C_{1}$-phenyl trans- $N$-phenyl 2-aziridinylmethyl radical

\begin{tabular}{ccccc} 
Center & Atomic & \multicolumn{3}{c}{ Coordinates (Angstroms) } \\
Number & Number & X & Y & Z \\
- \hdashline 1 & N & 0.000000 & 0.000000 & 0.000000 \\
2 & C & 0.000000 & 0.000000 & 1.442455 \\
3 & C & 1.326749 & 0.000000 & 0.623727
\end{tabular}




$\begin{array}{rrrrr}4 & \mathrm{C} & 2.196379 & -1.148559 & 0.462735 \\ 5 & \mathrm{H} & 1.788827 & -2.124600 & 0.228983 \\ 6 & \mathrm{H} & 3.246646 & -1.070533 & 0.720857 \\ 7 & \mathrm{H} & 1.824615 & 0.971354 & 0.667461 \\ 8 & \mathrm{H} & -0.184331 & 0.962737 & 1.926465 \\ 9 & \mathrm{C} & -0.526436 & -1.177580 & 2.186576 \\ 10 & \mathrm{C} & -0.456202 & 1.121972 & -0.729071 \\ 11 & \mathrm{C} & -0.971941 & -2.330920 & 1.527240 \\ 12 & \mathrm{C} & -1.447852 & -3.421282 & 2.255547 \\ 13 & \mathrm{C} & -1.486122 & -3.375153 & 3.650699 \\ 14 & \mathrm{C} & -1.049147 & -2.226937 & 4.314987 \\ 15 & \mathrm{C} & -0.574841 & -1.136454 & 3.587325 \\ 16 & \mathrm{H} & -0.237816 & -0.242992 & 4.108986 \\ 17 & \mathrm{H} & -1.080787 & -2.179004 & 5.400398 \\ 18 & \mathrm{H} & -1.858331 & -4.225099 & 4.216443 \\ 19 & \mathrm{H} & -1.795209 & -4.307775 & 1.731011 \\ 20 & \mathrm{H} & -0.953844 & -2.352924 & 0.442030 \\ 21 & \mathrm{C} & -1.701097 & 1.699253 & -0.439221 \\ 22 & \mathrm{H} & -2.294639 & 1.304225 & 0.380698 \\ 23 & \mathrm{C} & -2.173404 & 2.762749 & -1.207202 \\ 24 & \mathrm{H} & -3.139774 & 3.201783 & -0.972567 \\ 25 & \mathrm{C} & -1.415958 & 3.264580 & -2.267604 \\ 26 & \mathrm{H} & -1.786426 & 4.094947 & -2.861900 \\ 27 & \mathrm{C} & -0.177329 & 2.686995 & -2.556992 \\ 28 & \mathrm{C} & 0.302995 & 1.621942 & -1.797215 \\ 29 & \mathrm{H} & 1.262729 & 1.166759 & -2.025431 \\ 30 & \mathrm{H} & 0.421640 & 3.067527 & -3.380453\end{array}$

trans- $C_{1}$-phenyl $c i s-N$-phenyl 2-aziridinylmethyl radical

\begin{tabular}{ccrrr} 
Center & Atomic & \multicolumn{4}{c}{ Coordinates (Angstroms) } \\
Number & Number & X & Y & Z \\
---1 & N & 0.000000 & 0.000000 & 0.000000 \\
1 & C & 0.000000 & 0.000000 & 1.440971 \\
2 & $\mathrm{C}$ & 1.331889 & 0.000000 & 0.644778 \\
3 & $\mathrm{C}$ & 2.203113 & -1.154249 & 0.650736 \\
4 & $\mathrm{H}$ & 1.807765 & -2.160058 & 0.569548 \\
5 & $\mathrm{H}$ & 3.272620 & -1.025577 & 0.767275 \\
6 & $\mathrm{H}$ & 1.818880 & 0.971935 & 0.584253 \\
7 & $\mathrm{H}$ & -0.203761 & -0.955241 & 1.929182 \\
8 & $\mathrm{C}$ & -0.473615 & 1.202718 & 2.180027 \\
9 & $\mathrm{C}$ & -0.501497 & -1.093755 & -0.742103 \\
10 & $\mathrm{C}$ & 0.152160 & -1.499751 & -1.916522 \\
11 & $\mathrm{C}$ & -0.386727 & -2.517104 & -2.699851 \\
12 & & & &
\end{tabular}




$\begin{array}{llrrr}13 & \mathrm{C} & -1.584987 & -3.138235 & -2.335437 \\ 14 & \mathrm{C} & -2.239506 & -2.728551 & -1.173311 \\ 15 & \mathrm{C} & -1.703423 & -1.716357 & -0.376339 \\ 16 & \mathrm{H} & -2.222042 & -1.393131 & 0.521891 \\ 17 & \mathrm{H} & -3.174701 & -3.198451 & -0.879574 \\ 18 & \mathrm{H} & -2.002442 & -3.929544 & -2.951494 \\ 19 & \mathrm{H} & 0.132599 & -2.825378 & -3.603743 \\ 20 & \mathrm{H} & 1.080099 & -1.013461 & -2.202222 \\ 21 & \mathrm{C} & -0.764713 & 2.401384 & 1.514017 \\ 22 & \mathrm{C} & -1.175121 & 3.524241 & 2.231716 \\ 23 & \mathrm{C} & -1.300811 & 3.466247 & 3.621668 \\ 24 & \mathrm{C} & -1.016735 & 2.274031 & 4.291342 \\ 25 & \mathrm{C} & -0.606855 & 1.150305 & 3.574930 \\ 26 & \mathrm{H} & -0.387621 & 0.222846 & 4.099918 \\ 27 & \mathrm{H} & -1.117683 & 2.217796 & 5.372096 \\ 28 & \mathrm{H} & -1.622406 & 4.342141 & 4.178759 \\ 29 & \mathrm{H} & -1.403465 & 4.446361 & 1.703318 \\ 30 & \mathrm{H} & -0.679821 & 2.432976 & 0.431862\end{array}$

transition state of C-N cleavage of $c i s-C_{1}$-methyl trans- $N$-methyl 2-aziridinylmethyl radical

\begin{tabular}{|c|c|c|c|c|}
\hline \multirow{2}{*}{$\begin{array}{l}\text { Center } \\
\text { Number }\end{array}$} & \multirow{2}{*}{$\begin{array}{l}\text { Atomic } \\
\text { Number }\end{array}$} & \multicolumn{3}{|c|}{ Coordinates (Angstroms) } \\
\hline & & $X$ & $\mathrm{Y}$ & $\mathrm{Z}$ \\
\hline 1 & $\mathrm{~N}$ & 0.000000 & 0.000000 & 0.000000 \\
\hline 2 & $\mathrm{C}$ & 0.000000 & 0.000000 & 1.444259 \\
\hline 3 & $\mathrm{C}$ & 1.445762 & 0.000000 & 1.055326 \\
\hline 4 & $\mathrm{C}$ & 2.292978 & -1.106767 & 0.999927 \\
\hline 5 & $\mathrm{H}$ & 1.936157 & -2.115311 & 1.175660 \\
\hline 6 & $\mathrm{H}$ & 3.331379 & -0.989605 & 0.708047 \\
\hline 7 & $\mathrm{H}$ & 1.893912 & 0.984091 & 0.947218 \\
\hline 8 & $\mathrm{H}$ & -0.322424 & 0.933947 & 1.924561 \\
\hline 9 & $\mathrm{C}$ & -0.616328 & -1.221931 & 2.098227 \\
\hline 10 & $\mathrm{C}$ & -0.200088 & 1.305750 & -0.612511 \\
\hline 11 & $\mathrm{H}$ & -1.277389 & 1.435684 & -0.800161 \\
\hline 12 & $\mathrm{H}$ & 0.312211 & 1.347887 & -1.579808 \\
\hline 13 & $\mathrm{H}$ & 0.131458 & 2.161728 & -0.000239 \\
\hline 14 & $\mathrm{H}$ & -0.334262 & -2.135370 & 1.567662 \\
\hline 15 & $\mathrm{H}$ & -0.297929 & -1.306489 & 3.143697 \\
\hline 16 & $\mathrm{H}$ & -1.709457 & -1.148019 & 2.075060 \\
\hline
\end{tabular}

transition state of C-N cleavage of trans- $C_{1}$-methyl cis- $N$-methyl 2-aziridinylmethyl radical

$\begin{array}{lllcl}\text { Center } & \text { Atomic } & \text { Coordinates (Angstroms) } & \\ \text { Number } & \text { Number } & \mathrm{X} & \mathrm{Y} & \mathrm{Z}\end{array}$




$\begin{array}{rrrrr}1 & \mathrm{~N} & 0.000000 & 0.000000 & 0.000000 \\ 2 & \mathrm{C} & 0.000000 & 0.000000 & 1.441916 \\ 3 & \mathrm{C} & 1.438644 & 0.000000 & 1.031456 \\ 4 & \mathrm{C} & 2.280554 & -1.112867 & 1.080950 \\ 5 & \mathrm{H} & 1.915053 & -2.098596 & 1.350571 \\ 6 & \mathrm{H} & 3.322115 & -1.032261 & 0.787871 \\ 7 & \mathrm{H} & 1.877395 & 0.974991 & 0.845080 \\ 8 & \mathrm{H} & -0.323072 & -0.932443 & 1.924047 \\ 9 & \mathrm{C} & -0.574662 & 1.237001 & 2.107043 \\ 10 & \mathrm{C} & -0.321948 & -1.269881 & -0.636481 \\ 11 & \mathrm{H} & -0.145708 & -2.162547 & -0.017951 \\ 12 & \mathrm{H} & 0.241790 & -1.375125 & -1.569383 \\ 13 & \mathrm{H} & -1.391246 & -1.248657 & -0.892712 \\ 14 & \mathrm{H} & -1.669878 & 1.223360 & 2.062206 \\ 15 & \mathrm{H} & -0.272126 & 1.287576 & 3.159358 \\ 16 & \mathrm{H} & -0.227410 & 2.141213 & 1.597005\end{array}$

transition state of C-N cleavage of $c i s$ - $C_{l}$-formyl trans- $N$-methyl 2-aziridinylmethyl radical

\begin{tabular}{ccccc} 
Center & Atomic & \multicolumn{3}{c}{ Coordinates (Angstroms) } \\
Number & Number & X & Y & Z \\
- & & & & \\
1 & N & 0.000000 & 0.000000 & 0.000000 \\
2 & $\mathrm{C}$ & 0.000000 & 0.000000 & 1.448498 \\
3 & $\mathrm{C}$ & 1.438823 & 0.000000 & 1.027705 \\
4 & $\mathrm{C}$ & 2.268296 & -1.119751 & 0.891557 \\
5 & $\mathrm{H}$ & 1.885337 & -2.128618 & 0.992062 \\
6 & $\mathrm{H}$ & 3.313266 & -1.001116 & 0.625788 \\
7 & $\mathrm{H}$ & 1.890757 & 0.985828 & 0.959584 \\
8 & $\mathrm{H}$ & -0.309184 & 0.942827 & 1.905040 \\
9 & $\mathrm{C}$ & -0.618897 & -1.220230 & 2.098698 \\
10 & $\mathrm{C}$ & -0.176164 & 1.248257 & -0.612291 \\
11 & $\mathrm{O}$ & -0.559013 & 2.272425 & -0.070961 \\
12 & $\mathrm{H}$ & 0.034456 & 1.204311 & -1.694872 \\
13 & $\mathrm{H}$ & -0.350724 & -2.138862 & 1.569393 \\
14 & $\mathrm{H}$ & -1.710455 & -1.132645 & 2.080480 \\
15 & $\mathrm{H}$ & -0.299379 & -1.305404 & 3.143403
\end{tabular}

transition state of C-N cleavage of trans- $C_{l}$-formyl $c i s-N$-methyl 2-aziridinylmethyl radical

\begin{tabular}{ccccr} 
Center & Atomic & \multicolumn{4}{c}{ Coordinates } & \multicolumn{2}{c}{ (Angstroms) } \\
Number & Number & X & Y & Z \\
-1 & N & 0.000000 & 0.000000 & 0.000000
\end{tabular}




$\begin{array}{rrrrr}2 & \mathrm{C} & 0.000000 & 0.000000 & 1.440510 \\ 3 & \mathrm{C} & 1.445486 & 0.000000 & 1.029353 \\ 4 & \mathrm{C} & 2.256046 & -1.137407 & 1.049199 \\ 5 & \mathrm{H} & 3.309809 & -1.074633 & 0.799505 \\ 6 & \mathrm{H} & 1.839238 & -2.119715 & 1.248481 \\ 7 & \mathrm{H} & 1.899707 & 0.970403 & 0.857374 \\ 8 & \mathrm{H} & -0.334265 & -0.933650 & 1.900581 \\ 9 & \mathrm{C} & -0.563501 & 1.242170 & 2.102645 \\ 10 & \mathrm{C} & -0.361851 & -1.192674 & -0.632395 \\ 11 & \mathrm{H} & -0.042796 & -1.214510 & -1.688734 \\ 12 & \mathrm{O} & -1.013932 & -2.095677 & -0.136393 \\ 13 & \mathrm{H} & -0.217640 & 2.147392 & 1.592816 \\ 14 & \mathrm{H} & -0.260372 & 1.290250 & 3.154476 \\ 15 & \mathrm{H} & -1.657823 & 1.225751 & 2.060033\end{array}$

transition state of C-N cleavage of cis- $C_{I}$-phenyl trans- $N$-methyl 2-aziridinylmethyl radical

\begin{tabular}{|c|c|c|c|c|}
\hline \multirow{2}{*}{$\begin{array}{l}\text { Center } \\
\text { Number }\end{array}$} & \multirow{2}{*}{$\begin{array}{l}\text { Atomic } \\
\text { Number }\end{array}$} & \multicolumn{3}{|c|}{ Coordinates (Angstroms) } \\
\hline & & $\mathrm{X}$ & $\mathrm{Y}$ & $\mathrm{Z}$ \\
\hline 1 & $\mathrm{~N}$ & 0.000000 & 0.000000 & 0.000000 \\
\hline 2 & $\mathrm{C}$ & 0.000000 & 0.000000 & 1.443306 \\
\hline 3 & $\mathrm{C}$ & 1.425348 & 0.000000 & 0.977492 \\
\hline 4 & $\mathrm{C}$ & 2.280779 & -1.111955 & 0.890365 \\
\hline 5 & $\mathrm{H}$ & 1.918626 & -2.125525 & 1.018411 \\
\hline 6 & $\mathrm{H}$ & 3.321468 & -0.981164 & 0.614015 \\
\hline 7 & $\mathrm{H}$ & 1.873360 & 0.986920 & 0.900308 \\
\hline 8 & $\mathrm{C}$ & -0.607474 & -1.220645 & 2.104451 \\
\hline 9 & $\mathrm{H}$ & -0.286355 & 0.933536 & 1.938482 \\
\hline 10 & $\mathrm{C}$ & -0.240704 & 1.196994 & -0.700409 \\
\hline 11 & $\mathrm{C}$ & -1.078298 & 2.226109 & -0.230025 \\
\hline 12 & $\mathrm{C}$ & -1.337884 & 3.343790 & -1.024158 \\
\hline 13 & $\mathrm{C}$ & -0.759307 & 3.468421 & -2.287479 \\
\hline 14 & $\mathrm{C}$ & 0.074942 & 2.449844 & -2.762480 \\
\hline 15 & $\mathrm{C}$ & 0.324280 & 1.324914 & -1.985363 \\
\hline 16 & $\mathrm{H}$ & 0.959891 & 0.522688 & -2.348644 \\
\hline 17 & $\mathrm{H}$ & 0.526645 & 2.532418 & -3.747841 \\
\hline 18 & $\mathrm{H}$ & -0.959989 & 4.343697 & -2.898915 \\
\hline 19 & $\mathrm{H}$ & -1.998923 & 4.121464 & -0.649513 \\
\hline 20 & $\mathrm{H}$ & -1.554059 & 2.140187 & 0.742368 \\
\hline 21 & $\mathrm{H}$ & -0.346784 & -2.134013 & 1.563071 \\
\hline 22 & $\mathrm{H}$ & -1.700179 & -1.138526 & 2.111296 \\
\hline 23 & $\mathrm{H}$ & -0.261714 & -1.311410 & 3.140493 \\
\hline
\end{tabular}

transition state of C-N cleavage of trans- $C_{1}$-phenyl cis- $N$-methyl 2-aziridinylmethyl radical 


\begin{tabular}{|c|c|c|c|c|}
\hline \multirow{2}{*}{$\begin{array}{l}\text { Center } \\
\text { Number }\end{array}$} & \multirow{2}{*}{$\begin{array}{l}\text { Atomic } \\
\text { Number }\end{array}$} & \multicolumn{3}{|c|}{ Coordinates (Angstroms) } \\
\hline & & $\mathrm{X}$ & $\mathrm{Y}$ & $\mathrm{Z}$ \\
\hline 1 & $\mathrm{~N}$ & 0.000000 & 0.000000 & 0.000000 \\
\hline 2 & $\mathrm{C}$ & 0.000000 & 0.000000 & 1.440990 \\
\hline 3 & $\mathrm{C}$ & 1.422580 & 0.000000 & 0.968891 \\
\hline 4 & $\mathrm{C}$ & 2.255810 & -1.130316 & 0.994833 \\
\hline 5 & $\mathrm{H}$ & 1.856719 & -2.121825 & 1.182361 \\
\hline 6 & $\mathrm{H}$ & 3.302188 & -1.053495 & 0.720094 \\
\hline 7 & $\mathrm{H}$ & 1.874276 & 0.974445 & 0.812665 \\
\hline 8 & $\mathrm{H}$ & -0.298829 & -0.933461 & 1.930397 \\
\hline 9 & $\mathrm{C}$ & -0.561553 & 1.237391 & 2.113555 \\
\hline 10 & $\mathrm{C}$ & -0.373865 & -1.159908 & -0.702491 \\
\hline 11 & $\mathrm{C}$ & -1.392854 & -2.024575 & -0.263633 \\
\hline 12 & $\mathrm{C}$ & -1.796038 & -3.100722 & -1.055293 \\
\hline 13 & $\mathrm{C}$ & -1.182337 & -3.346196 & -2.283885 \\
\hline 14 & $\mathrm{C}$ & -0.165778 & -2.491719 & -2.725262 \\
\hline 15 & $\mathrm{C}$ & 0.228991 & -1.406933 & -1.950389 \\
\hline 16 & $\mathrm{H}$ & 1.009804 & -0.732880 & -2.289842 \\
\hline 17 & $\mathrm{H}$ & 0.317275 & -2.671245 & -3.682462 \\
\hline 18 & $\mathrm{H}$ & -1.494333 & -4.188994 & -2.894334 \\
\hline 19 & $\mathrm{H}$ & -2.595128 & -3.750052 & -0.706241 \\
\hline 20 & $\mathrm{H}$ & -1.890579 & -1.837887 & 0.683672 \\
\hline 21 & $\mathrm{H}$ & -1.657399 & 1.218715 & 2.098949 \\
\hline 22 & $\mathrm{H}$ & -0.231113 & 1.293563 & 3.157009 \\
\hline 23 & $\mathrm{H}$ & -0.233274 & 2.140920 & 1.589984 \\
\hline
\end{tabular}

transition state of C-N cleavage of $c i s$ - $C_{1}$-methyl trans- $N$-formyl 2-aziridinylmethyl radical

\begin{tabular}{ccccc} 
Center & Atomic & \multicolumn{3}{c}{ Coordinates (Angstroms) } \\
Number & Number & X & Y & Z \\
- & Nume & & \\
1 & $\mathrm{~N}$ & 0.000000 & 0.000000 & 0.000000 \\
2 & $\mathrm{C}$ & 0.000000 & 0.000000 & 1.443136 \\
3 & $\mathrm{C}$ & 1.457633 & 0.000000 & 1.073026 \\
4 & $\mathrm{C}$ & 2.303961 & -1.100053 & 1.009609 \\
5 & $\mathrm{H}$ & 1.952191 & -2.114882 & 1.156473 \\
6 & $\mathrm{H}$ & 3.346323 & -0.969192 & 0.739811 \\
7 & $\mathrm{H}$ & 1.890277 & 0.992235 & 0.983454 \\
8 & $\mathrm{H}$ & -0.340587 & 0.897369 & 1.972292 \\
9 & $\mathrm{C}$ & -0.572573 & -1.249093 & 2.068587 \\
10 & $\mathrm{C}$ & -0.223131 & 1.307984 & -0.601034 \\
11 & $\mathrm{H}$ & 0.129546 & 2.160551 & 0.001961 \\
12 & $\mathrm{H}$ & 0.256139 & 1.348156 & -1.584353
\end{tabular}




$\begin{array}{llrrr}13 & \mathrm{H} & -1.306457 & 1.435515 & -0.749360 \\ 14 & \mathrm{H} & -0.564745 & -2.144909 & 1.412582 \\ 15 & \mathrm{O} & -1.016177 & -1.281136 & 3.194431\end{array}$

transition state of C-N cleavage of trans- $C_{l}$-methyl $c i s$ - $N$-formyl 2-aziridinylmethyl radical

\begin{tabular}{|c|c|c|c|c|}
\hline \multirow{2}{*}{$\begin{array}{l}\text { Center } \\
\text { Number }\end{array}$} & \multirow{2}{*}{$\begin{array}{l}\text { Atomic } \\
\text { Number }\end{array}$} & \multicolumn{3}{|c|}{ Coordinates (Angstroms) } \\
\hline & & $\mathrm{X}$ & $\mathrm{Y}$ & $\mathrm{Z}$ \\
\hline 1 & $\mathrm{~N}$ & 0.000000 & 0.000000 & 0.000000 \\
\hline 2 & $\mathrm{C}$ & 0.000000 & 0.000000 & 1.441731 \\
\hline 3 & $\mathrm{C}$ & 1.454337 & 0.000000 & 1.054544 \\
\hline 4 & $\mathrm{C}$ & 2.276840 & -1.114633 & 1.144250 \\
\hline 5 & $\mathrm{H}$ & 1.899718 & -2.088053 & 1.439454 \\
\hline 6 & $\mathrm{H}$ & 3.324166 & -1.046827 & 0.869725 \\
\hline 7 & $\mathrm{H}$ & 1.893516 & 0.968677 & 0.842654 \\
\hline 8 & $\mathrm{H}$ & -0.345260 & -0.893702 & 1.972356 \\
\hline 9 & $\mathrm{C}$ & -0.507549 & 1.268586 & 2.081413 \\
\hline 10 & $\mathrm{C}$ & -0.344896 & -1.274260 & -0.616335 \\
\hline 11 & $\mathrm{H}$ & -0.118578 & -2.161844 & -0.009402 \\
\hline 12 & $\mathrm{H}$ & -1.427803 & -1.266050 & -0.807029 \\
\hline 13 & $\mathrm{H}$ & 0.165998 & -1.366046 & -1.579758 \\
\hline 14 & $\mathrm{O}$ & -1.008018 & 1.308884 & 3.182330 \\
\hline 15 & $\mathrm{H}$ & -0.370754 & 2.180123 & 1.459689 \\
\hline
\end{tabular}

transition state of C-N cleavage of $c i s$ - $C_{1}$-formyl trans- $N$-formyl 2-aziridinylmethyl radical

\begin{tabular}{crrrr} 
Center & Atomic & \multicolumn{3}{c}{ Coordinates (Angstroms) } \\
Number & Number & X & Y & $\mathrm{Z}$ \\
- & & & \\
1 & $\mathrm{~N}$ & 0.000000 & 0.000000 & 0.000000 \\
2 & $\mathrm{C}$ & 0.000000 & 0.000000 & 1.449182 \\
3 & $\mathrm{C}$ & 1.444773 & 0.000000 & 1.043265 \\
4 & $\mathrm{C}$ & 2.274381 & -1.114879 & 0.898069 \\
5 & $\mathrm{H}$ & 1.896174 & -2.128251 & 0.967778 \\
6 & $\mathrm{H}$ & 3.323000 & -0.984478 & 0.654218 \\
7 & $\mathrm{H}$ & 1.882763 & 0.992538 & 0.994146 \\
8 & $\mathrm{H}$ & -0.326157 & 0.910283 & 1.953987 \\
9 & $\mathrm{C}$ & -0.572872 & -1.254424 & 2.067023 \\
10 & $\mathrm{C}$ & -0.159953 & 1.265451 & -0.593051 \\
11 & $\mathrm{H}$ & -0.075459 & 1.207637 & -1.690935 \\
12 & $\mathrm{O}$ & -0.392583 & 2.310656 & -0.012241 \\
13 & $\mathrm{H}$ & -0.631189 & -2.131410 & 1.388293 \\
14 & $\mathrm{O}$ & -0.946852 & -1.304968 & 3.215664
\end{tabular}


transition state of C-N cleavage of trans- $C_{1}$-formyl $c i s-N$-formyl 2-aziridinylmethyl radical

\begin{tabular}{ccccc} 
Center & Atomic & \multicolumn{3}{c}{ Coordinates (Angstroms) } \\
Number & Number & X & Y & Z \\
-1 & N & 0.000000 & 0.000000 & 0.000000 \\
2 & $\mathrm{C}$ & 0.000000 & 0.000000 & 1.444582 \\
3 & $\mathrm{C}$ & 1.448889 & 0.000000 & 1.037781 \\
4 & $\mathrm{C}$ & 2.250857 & -1.139177 & 1.093452 \\
5 & $\mathrm{H}$ & 1.828159 & -2.116953 & 1.297702 \\
6 & $\mathrm{H}$ & 3.309933 & -1.075922 & 0.867951 \\
7 & $\mathrm{H}$ & 1.905863 & 0.965630 & 0.849346 \\
8 & $\mathrm{H}$ & -0.355862 & -0.895621 & 1.954864 \\
9 & $\mathrm{C}$ & -0.498212 & 1.284799 & 2.064669 \\
10 & $\mathrm{C}$ & -0.327686 & -1.226193 & -0.604464 \\
11 & $\mathrm{H}$ & -0.171149 & -1.189397 & -1.695405 \\
12 & $\mathrm{O}$ & -0.770000 & -2.211500 & -0.044425 \\
13 & $\mathrm{H}$ & -0.350509 & 2.188202 & 1.433995 \\
14 & $\mathrm{O}$ & -1.004013 & 1.338835 & 3.160569
\end{tabular}

transition state of C-N cleavage of $c i s-C_{1}$-phenyl trans- $N$-formyl 2-aziridinylmethyl radical

\begin{tabular}{|c|c|c|c|c|}
\hline \multirow{2}{*}{$\begin{array}{l}\text { Center } \\
\text { Number }\end{array}$} & \multirow{2}{*}{$\begin{array}{l}\text { Atomic } \\
\text { Number }\end{array}$} & \multicolumn{3}{|c|}{ Coordinates (Angstroms) } \\
\hline & & $\mathrm{X}$ & $\mathrm{Y}$ & $\mathrm{Z}$ \\
\hline 1 & $\mathrm{~N}$ & 0.000000 & 0.000000 & 0.000000 \\
\hline 2 & $\mathrm{C}$ & 0.000000 & 0.000000 & 1.441190 \\
\hline 3 & $\mathrm{C}$ & 1.435901 & 0.000000 & 0.985650 \\
\hline 4 & $\mathrm{C}$ & 2.292367 & -1.104652 & 0.893218 \\
\hline 5 & $\mathrm{H}$ & 1.936625 & -2.123431 & 0.994967 \\
\hline 6 & $\mathrm{H}$ & 3.334927 & -0.960645 & 0.632326 \\
\hline 7 & $\mathrm{H}$ & 1.868980 & 0.994384 & 0.927517 \\
\hline 8 & $\mathrm{H}$ & -0.301260 & 0.893369 & 1.993176 \\
\hline 9 & $\mathrm{C}$ & -0.558644 & -1.251970 & 2.070889 \\
\hline 10 & $\mathrm{C}$ & -0.246472 & 1.198805 & -0.696646 \\
\hline 11 & $\mathrm{C}$ & -0.976148 & 2.285501 & -0.179308 \\
\hline 12 & $\mathrm{C}$ & -1.230968 & 3.403062 & -0.974675 \\
\hline 13 & $\mathrm{C}$ & -0.754721 & 3.466957 & -2.284559 \\
\hline 14 & $\mathrm{C}$ & -0.029233 & 2.389597 & -2.805894 \\
\hline 15 & $\mathrm{C}$ & 0.215462 & 1.265528 & -2.026473 \\
\hline 16 & $\mathrm{H}$ & 0.766469 & 0.417326 & -2.421539 \\
\hline 17 & $\mathrm{H}$ & 0.339927 & 2.425752 & -3.827474 \\
\hline 18 & $\mathrm{H}$ & -0.951760 & 4.342494 & -2.896616 \\
\hline 19 & $\mathrm{H}$ & -1.807933 & 4.227883 & -0.564549 \\
\hline 20 & $\mathrm{H}$ & -1.374060 & 2.249605 & 0.829972 \\
\hline
\end{tabular}




$\begin{array}{lllll}21 & \mathrm{H} & -0.590473 & -2.138195 & 1.403081 \\ 22 & \mathrm{O} & -0.946439 & -1.294640 & 3.216610\end{array}$

transition state of C-N cleavage of trans- $C_{1}$-phenyl cis- $N$-formyl 2-aziridinylmethyl radical

\begin{tabular}{|c|c|c|c|c|}
\hline \multirow{2}{*}{$\begin{array}{l}\text { Center } \\
\text { Number }\end{array}$} & \multirow{2}{*}{$\begin{array}{l}\text { Atomic } \\
\text { Number }\end{array}$} & \multicolumn{3}{|c|}{ Coordinates (Angstroms) } \\
\hline & & $\mathrm{X}$ & $\mathrm{Y}$ & $\mathrm{Z}$ \\
\hline 1 & $\mathrm{~N}$ & 0.000000 & 0.000000 & 0.000000 \\
\hline 2 & $\mathrm{C}$ & 0.000000 & 0.000000 & 1.439475 \\
\hline 3 & $\mathrm{C}$ & 1.437830 & 0.000000 & 0.983783 \\
\hline 4 & $\mathrm{C}$ & 2.254701 & -1.131097 & 1.044957 \\
\hline 5 & $\mathrm{H}$ & 1.848492 & -2.114391 & 1.256408 \\
\hline 6 & $\mathrm{H}$ & 3.305281 & -1.063267 & 0.785042 \\
\hline 7 & $\mathrm{H}$ & 1.888931 & 0.970405 & 0.806974 \\
\hline 8 & $\mathrm{H}$ & -0.315808 & -0.893446 & 1.983318 \\
\hline 9 & $\mathrm{C}$ & -0.491769 & 1.270661 & 2.084547 \\
\hline 10 & $\mathrm{C}$ & -0.390113 & -1.162462 & -0.690618 \\
\hline 11 & $\mathrm{C}$ & -1.356461 & -2.063090 & -0.207993 \\
\hline 12 & $\mathrm{C}$ & -1.766319 & -3.141194 & -0.993058 \\
\hline 13 & $\mathrm{C}$ & -1.211019 & -3.350086 & -2.255834 \\
\hline 14 & $\mathrm{C}$ & -0.247658 & -2.458162 & -2.740279 \\
\hline 15 & $\mathrm{C}$ & 0.153338 & -1.371205 & -1.972126 \\
\hline 16 & $\mathrm{H}$ & 0.891885 & -0.666361 & -2.341861 \\
\hline 17 & $\mathrm{H}$ & 0.187228 & -2.609500 & -3.724759 \\
\hline 18 & $\mathrm{H}$ & -1.528457 & -4.194817 & -2.860582 \\
\hline 19 & $\mathrm{H}$ & -2.524675 & -3.820280 & -0.612214 \\
\hline 20 & $\mathrm{H}$ & -1.811496 & -1.905129 & 0.765210 \\
\hline 21 & $\mathrm{H}$ & -0.385923 & 2.177164 & 1.449920 \\
\hline 22 & $\mathrm{O}$ & -0.946062 & 1.316299 & 3.204993 \\
\hline
\end{tabular}

transition state of C-N cleavage of $\operatorname{cis}$ - $C_{1}$-methyl trans- $N$-phenyl 2-aziridinylmethyl radical

\begin{tabular}{ccccc} 
Center & Atomic & \multicolumn{3}{c}{ Coordinates (Angstroms) } \\
Number & Number & X & Y & Z \\
- & Nume & & \\
1 & $\mathrm{~N}$ & 0.000000 & 0.000000 & 0.000000 \\
2 & $\mathrm{C}$ & 0.000000 & 0.000000 & 1.440501 \\
3 & $\mathrm{C}$ & 1.458290 & 0.000000 & 1.063959 \\
4 & $\mathrm{C}$ & 2.288140 & -1.113889 & 1.020611 \\
5 & $\mathrm{H}$ & 1.908831 & -2.110806 & 1.215753 \\
6 & $\mathrm{H}$ & 3.329428 & -1.013661 & 0.732938 \\
7 & $\mathrm{H}$ & 1.903323 & 0.984801 & 0.950095 \\
8 & $\mathrm{H}$ & -0.314535 & 0.934262 & 1.924949 \\
9 & $\mathrm{C}$ & -0.591990 & -1.203475 & 2.125520
\end{tabular}




$\begin{array}{llrrc}10 & \mathrm{C} & -0.210679 & 1.305827 & -0.606367 \\ 11 & \mathrm{H} & -1.291376 & 1.438960 & -0.770168 \\ 12 & \mathrm{H} & 0.282654 & 1.347183 & -1.583213 \\ 13 & \mathrm{H} & 0.136570 & 2.158379 & 0.001313 \\ 14 & \mathrm{C} & -1.034709 & -2.314260 & 1.398742 \\ 15 & \mathrm{C} & -1.572683 & -3.420429 & 2.059848 \\ 16 & \mathrm{C} & -1.671854 & -3.429722 & 3.451930 \\ 17 & \mathrm{C} & -1.232027 & -2.323417 & 4.183112 \\ 18 & \mathrm{C} & -0.697175 & -1.218300 & 3.522681 \\ 19 & \mathrm{H} & -0.358028 & -0.357321 & 4.095459 \\ 20 & \mathrm{H} & -1.309566 & -2.320041 & 5.267333 \\ 21 & \mathrm{H} & -2.091718 & -4.290975 & 3.965026 \\ 22 & \mathrm{H} & -1.917291 & -4.275925 & 1.484317 \\ 23 & \mathrm{H} & -0.957467 & -2.291274 & 0.316605\end{array}$

transition state of C-N cleavage of trans- $C_{1}$-methyl $c i s$ - $N$-phenyl 2-aziridinylmethyl radical

\begin{tabular}{crrrr} 
Center & Atomic & \multicolumn{3}{c}{ Coordinates (Angstroms) } \\
Number & Number & X & Y & Z \\
- & N & 0.000000 & 0.000000 & 0.000000 \\
2 & $\mathrm{C}$ & 0.000000 & 0.000000 & 1.438853 \\
3 & $\mathrm{C}$ & 1.453471 & 0.000000 & 1.045125 \\
4 & $\mathrm{C}$ & 2.288869 & -1.109697 & 1.108056 \\
5 & $\mathrm{H}$ & 3.335315 & -1.029435 & 0.832746 \\
6 & $\mathrm{H}$ & 1.920993 & -2.093279 & 1.382284 \\
7 & $\mathrm{H}$ & 1.882469 & 0.977461 & 0.854494 \\
8 & $\mathrm{H}$ & -0.312166 & -0.934492 & 1.923811 \\
9 & $\mathrm{C}$ & -0.554315 & 1.213541 & 2.136917 \\
10 & $\mathrm{C}$ & -0.335786 & -1.268902 & -0.628657 \\
11 & $\mathrm{H}$ & 0.204282 & -1.370339 & -1.575600 \\
12 & $\mathrm{H}$ & -0.139760 & -2.159716 & -0.014133 \\
13 & $\mathrm{H}$ & -1.411929 & -1.251125 & -0.854945 \\
14 & $\mathrm{C}$ & -0.902874 & 2.365183 & 1.422360 \\
15 & $\mathrm{C}$ & -1.389970 & 3.488883 & 2.091922 \\
16 & $\mathrm{C}$ & -1.532961 & 3.474875 & 3.480499 \\
17 & $\mathrm{C}$ & -1.188792 & 2.327281 & 4.198699 \\
18 & $\mathrm{C}$ & -0.703981 & 1.203920 & 3.529685 \\
19 & $\mathrm{H}$ & -0.438892 & 0.310981 & 4.092405 \\
20 & $\mathrm{H}$ & -1.301648 & 2.305654 & 5.279605 \\
21 & $\mathrm{H}$ & -1.913793 & 4.350144 & 4.000236 \\
22 & $\mathrm{H}$ & -1.662271 & 4.376401 & 1.526206 \\
23 & $\mathrm{H}$ & -0.795457 & 2.361348 & 0.342227 \\
& & & &
\end{tabular}

transition state of C-N cleavage of $c i s-C_{1}$-formyl trans- $N$-phenyl 2-aziridinylmethyl radical 


\begin{tabular}{|c|c|c|c|c|}
\hline \multirow{2}{*}{$\begin{array}{l}\text { Center } \\
\text { Number }\end{array}$} & \multirow{2}{*}{$\begin{array}{l}\text { Atomic } \\
\text { Number }\end{array}$} & \multicolumn{3}{|c|}{ Coordinates (Angstroms) } \\
\hline & & $X$ & $\mathrm{Y}$ & $\mathrm{Z}$ \\
\hline 1 & $\mathrm{~N}$ & 0.000000 & 0.000000 & 0.000000 \\
\hline 2 & $\mathrm{C}$ & 0.000000 & 0.000000 & 1.447185 \\
\hline 3 & $\mathrm{C}$ & 1.446035 & 0.000000 & 1.027948 \\
\hline 4 & $\mathrm{C}$ & 2.260602 & -1.129215 & 0.907641 \\
\hline 5 & $\mathrm{H}$ & 1.858823 & -2.127292 & 1.041312 \\
\hline 6 & $\mathrm{H}$ & 3.306376 & -1.027979 & 0.638144 \\
\hline 7 & $\mathrm{H}$ & 1.894658 & 0.986345 & 0.951364 \\
\hline 8 & $\mathrm{H}$ & -0.300720 & 0.943817 & 1.907453 \\
\hline 9 & $\mathrm{C}$ & -0.589819 & -1.206688 & 2.122882 \\
\hline 10 & $\mathrm{C}$ & -0.161474 & 1.257539 & -0.600698 \\
\hline 11 & $\mathrm{H}$ & -0.030275 & 1.202581 & -1.694861 \\
\hline 12 & $\mathrm{O}$ & -0.441425 & 2.301471 & -0.035756 \\
\hline 13 & $\mathrm{C}$ & -0.684789 & -1.224096 & 3.520864 \\
\hline 14 & $\mathrm{C}$ & -1.215347 & -2.330898 & 4.181698 \\
\hline 15 & $\mathrm{C}$ & -1.662518 & -3.434151 & 3.450494 \\
\hline 16 & $\mathrm{H}$ & -2.080256 & -4.295809 & 3.964351 \\
\hline 17 & $\mathrm{C}$ & -1.576833 & -3.420675 & 2.057696 \\
\hline 18 & $\mathrm{C}$ & -1.042647 & -2.313283 & 1.395564 \\
\hline 19 & $\mathrm{H}$ & -0.982472 & -2.288520 & 0.312358 \\
\hline 20 & $\mathrm{H}$ & -1.931001 & -4.272081 & 1.482297 \\
\hline 21 & $\mathrm{H}$ & -1.285116 & -2.330302 & 5.266254 \\
\hline 22 & $\mathrm{H}$ & -0.343426 & -0.363809 & 4.092765 \\
\hline
\end{tabular}

transition state of C-N cleavage of trans- $C_{l}$-formyl $c i s-N$-phenyl 2-aziridinylmethyl radical

\begin{tabular}{ccccc} 
Center & Atomic & \multicolumn{3}{c}{ Coordinates (Angstroms) } \\
Number & Number & X & Y & Z \\
----------1000 & \\
1 & N & 0.000000 & 0.000000 & 0.000000 \\
2 & $\mathrm{C}$ & 0.000000 & 0.000000 & 1.440170 \\
3 & $\mathrm{C}$ & 1.454763 & 0.000000 & 1.032049 \\
4 & $\mathrm{C}$ & 2.262860 & -1.135402 & 1.061338 \\
5 & $\mathrm{H}$ & 3.321897 & -1.067737 & 0.836177 \\
6 & $\mathrm{H}$ & 1.843898 & -2.118994 & 1.248119 \\
7 & $\mathrm{H}$ & 1.900633 & 0.973957 & 0.863028 \\
8 & $\mathrm{H}$ & -0.322692 & -0.935147 & 1.904557 \\
9 & $\mathrm{C}$ & -0.535940 & 1.223256 & 2.132036 \\
10 & $\mathrm{C}$ & -0.334813 & -1.211895 & -0.617276 \\
11 & $\mathrm{O}$ & -0.868757 & -2.169034 & -0.084907 \\
12 & $\mathrm{H}$ & -0.098331 & -1.199398 & -1.694953 \\
13 & $\mathrm{C}$ & -0.856729 & 2.385421 & 1.421291
\end{tabular}




$\begin{array}{lllll}14 & \mathrm{C} & -1.325597 & 3.514174 & 2.094912 \\ 15 & \mathrm{C} & -1.477120 & 3.493805 & 3.482566 \\ 16 & \mathrm{C} & -1.161690 & 2.335441 & 4.196268 \\ 17 & \mathrm{C} & -0.695637 & 1.206480 & 3.523498 \\ 18 & \mathrm{H} & -0.456893 & 0.303081 & 4.080678 \\ 19 & \mathrm{H} & -1.283823 & 2.308996 & 5.275845 \\ 20 & \mathrm{H} & -1.844619 & 4.373002 & 4.005023 \\ 21 & \mathrm{H} & -1.578162 & 4.409776 & 1.533216 \\ 22 & \mathrm{H} & -0.748012 & 2.388839 & 0.341263\end{array}$

transition state of C-N cleavage of $c$ is- $C_{l}$-phenyl trans- $N$-phenyl 2-aziridinylmethyl radical

\begin{tabular}{crrrr} 
Center & Atomic & \multicolumn{3}{c}{ Coordinates (Angstroms) } \\
Number & Number & X & Y & Z \\
-------------1 & \\
1 & N & 0.000000 & 0.000000 & 0.000000 \\
2 & $\mathrm{C}$ & 0.000000 & 0.000000 & 1.439590 \\
3 & $\mathrm{C}$ & 1.439270 & 0.000000 & 0.987002 \\
4 & $\mathrm{C}$ & 2.277623 & -1.118597 & 0.915375 \\
5 & $\mathrm{H}$ & 1.896164 & -2.120476 & 1.076724 \\
6 & $\mathrm{H}$ & 3.319245 & -1.006375 & 0.634660 \\
7 & $\mathrm{H}$ & 1.882570 & 0.988106 & 0.900458 \\
8 & $\mathrm{H}$ & -0.277593 & 0.933846 & 1.939245 \\
9 & $\mathrm{C}$ & -0.582925 & -1.202997 & 2.128406 \\
10 & $\mathrm{C}$ & -0.249750 & 1.199143 & -0.691816 \\
11 & $\mathrm{C}$ & -1.094549 & 2.218370 & -0.212654 \\
12 & $\mathrm{C}$ & -1.359257 & 3.341368 & -0.997223 \\
13 & $\mathrm{C}$ & -0.778962 & 3.480658 & -2.258311 \\
14 & $\mathrm{C}$ & 0.061466 & 2.471299 & -2.741857 \\
15 & $\mathrm{C}$ & 0.316203 & 1.340638 & -1.974792 \\
16 & $\mathrm{H}$ & 0.957183 & 0.545550 & -2.344195 \\
17 & $\mathrm{H}$ & 0.513917 & 2.565482 & -3.725760 \\
18 & $\mathrm{H}$ & -0.983241 & 4.360561 & -2.861824 \\
19 & $\mathrm{H}$ & -2.026046 & 4.111353 & -0.617174 \\
20 & $\mathrm{H}$ & -1.573693 & 2.118536 & 0.756555 \\
21 & $\mathrm{C}$ & -1.051425 & -2.306488 & 1.406522 \\
22 & $\mathrm{C}$ & -1.577909 & -3.413487 & 2.075121 \\
23 & $\mathrm{C}$ & -1.640530 & -3.430373 & 3.469269 \\
24 & $\mathrm{C}$ & -1.176166 & -2.330813 & 4.195356 \\
25 & $\mathrm{C}$ & -0.652585 & -1.224691 & 3.527764 \\
26 & $\mathrm{H}$ & -0.294269 & -0.368869 & 4.096524 \\
27 & $\mathrm{H}$ & -1.225909 & -2.333263 & 5.281132 \\
28 & $\mathrm{H}$ & -2.052084 & -4.292191 & 3.988000 \\
29 & $\mathrm{H}$ & -1.943704 & -4.262912 & 1.503887 \\
30 & $\mathrm{H}$ & -1.004670 & -2.278553 & 0.322675 \\
& & & &
\end{tabular}


transition state of C-N cleavage of trans- $C_{1}$-phenyl cis- $N$-phenyl 2-aziridinylmethyl radical

\begin{tabular}{|c|c|c|c|c|}
\hline \multirow{2}{*}{$\begin{array}{l}\text { Center } \\
\text { Number }\end{array}$} & \multirow{2}{*}{$\begin{array}{l}\text { Atomic } \\
\text { Number }\end{array}$} & \multicolumn{3}{|c|}{ Coordinates (Angstroms) } \\
\hline & & $X$ & $\mathrm{Y}$ & $\mathrm{Z}$ \\
\hline 1 & $\mathrm{~N}$ & 0.000000 & 0.000000 & 0.000000 \\
\hline 2 & $\mathrm{C}$ & 0.000000 & 0.000000 & 1.438147 \\
\hline 3 & $\mathrm{C}$ & 1.438299 & 0.000000 & 0.981781 \\
\hline 4 & $\mathrm{C}$ & 2.264196 & -1.127478 & 1.017942 \\
\hline 5 & $\mathrm{H}$ & 1.863693 & -2.117065 & 1.213058 \\
\hline 6 & $\mathrm{H}$ & 3.314530 & -1.052165 & 0.758178 \\
\hline 7 & $\mathrm{H}$ & 1.879209 & 0.977751 & 0.821989 \\
\hline 8 & $\mathrm{C}$ & -0.542419 & 1.212574 & 2.142484 \\
\hline 9 & $\mathrm{H}$ & -0.286220 & -0.935270 & 1.931092 \\
\hline 10 & $\mathrm{C}$ & -0.385050 & -1.160670 & -0.693036 \\
\hline 11 & $\mathrm{C}$ & -1.396864 & -2.025354 & -0.236859 \\
\hline 12 & $\mathrm{C}$ & -1.806832 & -3.105304 & -1.019382 \\
\hline 13 & $\mathrm{C}$ & -1.207632 & -3.354057 & -2.254597 \\
\hline 14 & $\mathrm{C}$ & -0.199098 & -2.498950 & -2.713012 \\
\hline 15 & $\mathrm{C}$ & 0.202503 & -1.409940 & -1.947849 \\
\hline 16 & $\mathrm{H}$ & 0.977154 & -0.735110 & -2.299594 \\
\hline 17 & $\mathrm{H}$ & 0.271764 & -2.681103 & -3.675704 \\
\hline 18 & $\mathrm{H}$ & -1.525105 & -4.200100 & -2.857635 \\
\hline 19 & $\mathrm{H}$ & -2.600578 & -3.754597 & -0.658568 \\
\hline 20 & $\mathrm{H}$ & -1.885944 & -1.833384 & 0.713748 \\
\hline 21 & $\mathrm{C}$ & -0.688635 & 1.193906 & 3.535569 \\
\hline 22 & $\mathrm{C}$ & -1.160254 & 2.317272 & 4.213857 \\
\hline 23 & $\mathrm{C}$ & -1.494375 & 3.473304 & 3.504691 \\
\hline 24 & $\mathrm{C}$ & -1.355209 & 3.496074 & 2.115699 \\
\hline 25 & $\mathrm{C}$ & -0.881845 & 2.372535 & 1.436662 \\
\hline 26 & $\mathrm{H}$ & -0.780254 & 2.376255 & 0.355864 \\
\hline 27 & $\mathrm{H}$ & -1.620803 & 4.390012 & 1.557153 \\
\hline 28 & $\mathrm{H}$ & -1.865320 & 4.348435 & 4.031622 \\
\hline 29 & $\mathrm{H}$ & -1.271108 & 2.288746 & 5.294738 \\
\hline 30 & $\mathrm{H}$ & -0.431662 & 0.294065 & 4.090942 \\
\hline
\end{tabular}

transition state of C-C cleavage of $c i s$ - $C_{1}$-methyl trans- $N$-methyl 2-aziridinylmethyl radical

\begin{tabular}{ccccc} 
Center & Atomic & \multicolumn{4}{c}{ Coordinates (Angstroms) } \\
Number & Number & X & Y & Z \\
\hdashline 1 & N & 0.000000 & 0.000000 & 0.000000 \\
2 & C & 0.000000 & 0.000000 & 1.422406 \\
3 & C & 1.423213 & 0.000000 & 0.204632
\end{tabular}




$\begin{array}{rrrrr}4 & \mathrm{C} & 2.208626 & -1.107428 & -0.035803 \\ 5 & \mathrm{H} & 1.769145 & -2.042776 & -0.364529 \\ 6 & \mathrm{H} & 3.274336 & -1.080590 & 0.166041 \\ 7 & \mathrm{H} & 1.893035 & 0.968427 & 0.402328 \\ 8 & \mathrm{C} & -0.342985 & -1.243842 & 2.176013 \\ 9 & \mathrm{H} & -0.056551 & 0.967546 & 1.929306 \\ 10 & \mathrm{C} & -0.549878 & 1.178842 & -0.653884 \\ 11 & \mathrm{H} & -0.177906 & 2.126123 & -0.220228 \\ 12 & \mathrm{H} & -1.640955 & 1.163626 & -0.561041 \\ 13 & \mathrm{H} & -0.284275 & 1.157788 & -1.715579 \\ 14 & \mathrm{H} & -1.431071 & -1.414830 & 2.189884 \\ 15 & \mathrm{H} & 0.119634 & -2.121715 & 1.713028 \\ 16 & \mathrm{H} & -0.002601 & -1.179837 & 3.215495\end{array}$

transition state of C-C cleavage of trans- $C_{1}$-methyl cis- $N$-methyl 2-aziridinylmethyl radical

\begin{tabular}{|c|c|c|c|c|}
\hline \multirow{2}{*}{$\begin{array}{l}\text { Center } \\
\text { Number }\end{array}$} & \multirow{2}{*}{$\begin{array}{l}\text { Atomic } \\
\text { Number }\end{array}$} & \multicolumn{3}{|c|}{ Coordinates (Angstroms) } \\
\hline & & $\mathrm{X}$ & $\mathrm{Y}$ & $\mathrm{Z}$ \\
\hline 1 & $\mathrm{~N}$ & 0.000000 & 0.000000 & 0.000000 \\
\hline 2 & $\mathrm{C}$ & 0.000000 & 0.000000 & 1.427701 \\
\hline 3 & $\mathrm{C}$ & 1.421841 & 0.000000 & 0.220672 \\
\hline 4 & $\mathrm{C}$ & 2.281932 & -1.067120 & 0.067709 \\
\hline 5 & $\mathrm{H}$ & 3.348056 & -0.921566 & 0.201290 \\
\hline 6 & $\mathrm{H}$ & 1.941611 & -2.068770 & -0.169692 \\
\hline 7 & $\mathrm{H}$ & 1.836747 & 1.001016 & 0.322459 \\
\hline 8 & $\mathrm{H}$ & -0.055347 & -0.968117 & 1.928585 \\
\hline 9 & $\mathrm{C}$ & -0.364951 & 1.244648 & 2.173236 \\
\hline 10 & $\mathrm{C}$ & -0.588351 & -1.157818 & -0.658557 \\
\hline 11 & $\mathrm{H}$ & -0.243356 & -1.191556 & -1.696853 \\
\hline 12 & $\mathrm{H}$ & -0.342425 & -2.119932 & -0.177162 \\
\hline 13 & $\mathrm{H}$ & -1.677032 & -1.043982 & -0.650313 \\
\hline 14 & $\mathrm{H}$ & 0.036723 & 2.134910 & 1.676245 \\
\hline 15 & $\mathrm{H}$ & 0.011436 & 1.217863 & 3.201831 \\
\hline 16 & $\mathrm{H}$ & -1.458968 & 1.374076 & 2.221823 \\
\hline
\end{tabular}

transition state of C-C cleavage of $c i s$ - $C_{1}$-formyl trans- $N$-methyl 2-aziridinylmethyl radical

\begin{tabular}{ccccc} 
Center & Atomic & \multicolumn{4}{c}{ Coordinates (Angstroms) } \\
Number & Number & X & Y & Z \\
- \hdashline-----100000 \\
1 & N & 0.000000 & 0.000000 & 0.000000 \\
2 & C & 0.000000 & 0.000000 & 1.412678 \\
3 & C & 1.420154 & 0.000000 & 0.191306 \\
4 & C & 2.201207 & -1.117893 & -0.054141
\end{tabular}




$\begin{array}{rrrrr}5 & \mathrm{H} & 1.755966 & -2.059639 & -0.355140 \\ 6 & \mathrm{H} & 3.270863 & -1.085485 & 0.121998 \\ 7 & \mathrm{H} & 1.890627 & 0.970786 & 0.346104 \\ 8 & \mathrm{H} & -0.131878 & 0.967629 & 1.897050 \\ 9 & \mathrm{C} & -0.322720 & -1.246339 & 2.170707 \\ 10 & \mathrm{C} & -0.703615 & 0.883236 & -0.800404 \\ 11 & \mathrm{O} & -1.759680 & 1.396567 & -0.492970 \\ 12 & \mathrm{H} & -0.207039 & 1.033062 & -1.777714 \\ 13 & \mathrm{H} & 0.090880 & -2.130112 & 1.675450 \\ 14 & \mathrm{H} & -1.412452 & -1.381027 & 2.240857 \\ 15 & \mathrm{H} & 0.074165 & -1.196969 & 3.190341\end{array}$

transition state of C-C cleavage of trans- $C_{1}$-formyl cis- $N$-methyl 2-aziridinylmethyl radical

\begin{tabular}{ccrcc} 
Center & Atomic & \multicolumn{3}{c}{ Coordinates (Angstroms) } \\
Number & Number & X & Y & Z \\
-1 & N & 0.000000 & 0.000000 & 0.000000 \\
2 & $\mathrm{C}$ & 0.000000 & 0.000000 & 1.415073 \\
3 & $\mathrm{C}$ & 1.416211 & 0.000000 & 0.184537 \\
4 & $\mathrm{C}$ & 2.220125 & -1.115469 & 0.020035 \\
5 & $\mathrm{H}$ & 3.296575 & -1.028787 & 0.114259 \\
6 & $\mathrm{H}$ & 1.800181 & -2.103692 & -0.138696 \\
7 & $\mathrm{H}$ & 1.864020 & 0.989408 & 0.243637 \\
8 & $\mathrm{H}$ & -0.109700 & -0.969899 & 1.896106 \\
9 & $\mathrm{C}$ & -0.346442 & 1.246779 & 2.165128 \\
10 & $\mathrm{C}$ & -0.721282 & -0.843162 & -0.823570 \\
11 & $\mathrm{H}$ & -0.219335 & -0.982131 & -1.799470 \\
12 & $\mathrm{O}$ & -1.796904 & -1.330084 & -0.539907 \\
13 & $\mathrm{H}$ & 0.019624 & 2.139385 & 1.646557 \\
14 & $\mathrm{H}$ & 0.071269 & 1.228670 & 3.177323 \\
15 & $\mathrm{H}$ & -1.439353 & 1.348329 & 2.255385
\end{tabular}

transition state of C-C cleavage of $c i s$ - $C_{1}$-phenyl trans- $N$-methyl 2-aziridinylmethyl radical

\begin{tabular}{ccccc} 
Center & Atomic & \multicolumn{3}{c}{ Coordinates (Angstroms) } \\
Number & Number & X & Y & Z \\
---1 & N & 0.000000 & 0.000000 & 0.000000 \\
1 & N & 0.000000 & 0.000000 & 1.418886 \\
2 & C & 1.421569 & 0.000000 & 0.194453 \\
3 & C & 2.201900 & -1.112830 & -0.045850 \\
4 & C & 1.758341 & -2.050480 & -0.362104 \\
5 & H & 3.268538 & -1.086521 & 0.150287 \\
6 & H & 1.889165 & 0.971891 & 0.365533 \\
7 & H & & &
\end{tabular}




$\begin{array}{rrrrr}8 & \mathrm{H} & -0.087758 & 0.968047 & 1.916892 \\ 9 & \mathrm{C} & -0.318442 & -1.248113 & 2.176755 \\ 10 & \mathrm{C} & -0.628080 & 1.020531 & -0.743452 \\ 11 & \mathrm{C} & -1.874657 & 1.525156 & -0.343115 \\ 12 & \mathrm{C} & -2.516355 & 2.493349 & -1.113397 \\ 13 & \mathrm{C} & -1.929955 & 2.973150 & -2.286490 \\ 14 & \mathrm{C} & -0.689008 & 2.469281 & -2.683934 \\ 15 & \mathrm{C} & -0.037717 & 1.501441 & -1.922292 \\ 16 & \mathrm{H} & 0.925360 & 1.107404 & -2.233683 \\ 17 & \mathrm{H} & -0.220421 & 2.833529 & -3.594706 \\ 18 & \mathrm{H} & -2.432712 & 3.729208 & -2.882672 \\ 19 & \mathrm{H} & -3.482138 & 2.875159 & -0.791993 \\ 20 & \mathrm{H} & -2.337829 & 1.148842 & 0.564878 \\ 21 & \mathrm{H} & 0.129267 & -2.123452 & 1.695595 \\ 22 & \mathrm{H} & 0.055500 & -1.185910 & 3.204612 \\ 23 & \mathrm{H} & -1.405252 & -1.418655 & 2.226492\end{array}$

transition state of C-C cleavage of trans- $C_{l}$-phenyl cis- $N$-methyl 2-aziridinylmethyl radical

\begin{tabular}{crrrr} 
Center & Atomic & \multicolumn{3}{c}{ Coordinates (Angstroms) } \\
Number & Number & X & Y & Z \\
-----------1 & \\
1 & $\mathrm{~N}$ & 0.000000 & 0.000000 & 0.000000 \\
2 & $\mathrm{C}$ & 0.000000 & 0.000000 & 1.419376 \\
3 & $\mathrm{C}$ & 1.425636 & 0.000000 & 0.175678 \\
4 & $\mathrm{C}$ & 2.256352 & -1.090843 & 0.050613 \\
5 & $\mathrm{H}$ & 1.873751 & -2.086493 & -0.144914 \\
6 & $\mathrm{H}$ & 3.327599 & -0.969453 & 0.166445 \\
7 & $\mathrm{H}$ & 1.852393 & 0.997828 & 0.250010 \\
8 & $\mathrm{H}$ & -0.044030 & -0.967371 & 1.920454 \\
9 & $\mathrm{C}$ & -0.342486 & 1.248409 & 2.169128 \\
10 & $\mathrm{C}$ & -0.693900 & -0.959844 & -0.765679 \\
11 & $\mathrm{C}$ & -1.885726 & -1.523958 & -0.286487 \\
12 & $\mathrm{C}$ & -2.616182 & -2.405401 & -1.083580 \\
13 & $\mathrm{C}$ & -2.171152 & -2.743917 & -2.361663 \\
14 & $\mathrm{C}$ & -0.984024 & -2.180623 & -2.839271 \\
15 & $\mathrm{C}$ & -0.249481 & -1.294737 & -2.056088 \\
16 & $\mathrm{H}$ & 0.670679 & -0.859157 & -2.432445 \\
17 & $\mathrm{H}$ & -0.625195 & -2.431780 & -3.834297 \\
18 & $\mathrm{H}$ & -2.739355 & -3.434605 & -2.978018 \\
19 & $\mathrm{H}$ & -3.539126 & -2.830470 & -0.697076 \\
20 & $\mathrm{H}$ & -2.242290 & -1.260900 & 0.705358 \\
21 & $\mathrm{H}$ & -1.434303 & 1.369982 & 2.263355 \\
22 & $\mathrm{H}$ & 0.075799 & 1.230753 & 3.181485 \\
23 & $\mathrm{H}$ & 0.030757 & 2.137032 & 1.648276
\end{tabular}


transition state of C-C cleavage of $c i s$ - $C_{1}$-methyl trans- $N$-formyl 2-aziridinylmethyl radical

\begin{tabular}{ccccc} 
Center & Atomic & \multicolumn{3}{c}{ Coordinates (Angstroms) } \\
Number & Number & X & Y & Z \\
$-y$ & N & 0.000000 & 0.000000 & 0.000000 \\
2 & $\mathrm{C}$ & 0.000000 & 0.000000 & 1.427349 \\
3 & $\mathrm{C}$ & 1.395855 & 0.000000 & 0.320745 \\
4 & $\mathrm{C}$ & 2.216696 & -1.106149 & 0.081711 \\
5 & $\mathrm{H}$ & 1.800120 & -2.039376 & -0.280200 \\
6 & $\mathrm{H}$ & 3.269827 & -1.069888 & 0.337507 \\
7 & $\mathrm{H}$ & 1.865307 & 0.968191 & 0.520495 \\
8 & $\mathrm{H}$ & -0.049349 & 0.945424 & 1.972326 \\
9 & $\mathrm{C}$ & -0.301298 & -1.221053 & 2.168532 \\
10 & $\mathrm{C}$ & -0.531188 & 1.190328 & -0.654410 \\
11 & $\mathrm{H}$ & -0.164580 & 2.128972 & -0.202936 \\
12 & $\mathrm{H}$ & -0.243316 & 1.173343 & -1.709639 \\
13 & $\mathrm{H}$ & -1.622905 & 1.173191 & -0.583803 \\
14 & $\mathrm{H}$ & -0.352248 & -2.140734 & 1.548370 \\
15 & $\mathrm{O}$ & -0.474865 & -1.246538 & 3.377082
\end{tabular}

transition state of C-C cleavage of trans- $C_{l}$-methyl $c i s-N$-formyl 2-aziridinylmethyl radical

\begin{tabular}{ccccc} 
Center & Atomic & \multicolumn{3}{c}{ Coordinates (Angstroms) } \\
Number & Number & X & Y & Z \\
- & N & 0.000000 & 0.000000 & 0.000000 \\
1 & $\mathrm{C}$ & 0.000000 & 0.000000 & 1.428224 \\
2 & $\mathrm{C}$ & 1.398961 & 0.000000 & 0.337669 \\
3 & $\mathrm{C}$ & 2.253662 & -1.102466 & 0.334160 \\
4 & $\mathrm{H}$ & 1.900949 & -2.117636 & 0.194475 \\
5 & $\mathrm{H}$ & 3.314463 & -0.957102 & 0.501699 \\
6 & $\mathrm{H}$ & 1.840637 & 0.993940 & 0.343265 \\
7 & $\mathrm{H}$ & -0.076570 & -0.944186 & 1.968691 \\
8 & $\mathrm{C}$ & -0.223692 & 1.235774 & 2.168668 \\
9 & $\mathrm{C}$ & -0.573345 & -1.168541 & -0.660104 \\
10 & $\mathrm{H}$ & -0.359873 & -2.121730 & -0.150906 \\
11 & $\mathrm{H}$ & -1.658739 & -1.037959 & -0.695990 \\
12 & $\mathrm{H}$ & -0.187771 & -1.220526 & -1.682433 \\
13 & $\mathrm{H}$ & -0.257388 & 2.150807 & 1.538878 \\
14 & $\mathrm{O}$ & -0.345726 & 1.281968 & 3.385136 \\
15 & & &
\end{tabular}

transition state of C-C cleavage of cis- $_{l}$-formyl trans- $N$-formyl 2-aziridinylmethyl radical 


\begin{tabular}{|c|c|c|c|c|}
\hline \multirow{2}{*}{$\begin{array}{l}\text { Center } \\
\text { Number }\end{array}$} & \multirow{2}{*}{$\begin{array}{l}\text { Atomic } \\
\text { Number }\end{array}$} & \multicolumn{3}{|c|}{ Coordinates (Angstroms) } \\
\hline & & $X$ & $\mathrm{Y}$ & $\mathrm{Z}$ \\
\hline 1 & $\mathrm{~N}$ & 0.000000 & 0.000000 & 0.000000 \\
\hline 2 & $\mathrm{C}$ & 0.000000 & 0.000000 & 1.417386 \\
\hline 3 & $\mathrm{C}$ & 1.397349 & 0.000000 & 0.302092 \\
\hline 4 & $\mathrm{C}$ & 2.214524 & -1.112574 & 0.048410 \\
\hline 5 & $\mathrm{H}$ & 1.792541 & -2.048295 & -0.300431 \\
\hline 6 & $\mathrm{H}$ & 3.272913 & -1.071529 & 0.279246 \\
\hline 7 & $\mathrm{H}$ & 1.866684 & 0.969235 & 0.472968 \\
\hline 8 & $\mathrm{H}$ & -0.124555 & 0.943924 & 1.945669 \\
\hline 9 & $\mathrm{C}$ & -0.260352 & -1.242012 & 2.154039 \\
\hline 10 & $\mathrm{C}$ & -0.664006 & 0.941567 & -0.784648 \\
\hline 11 & $\mathrm{O}$ & -1.698403 & 1.482107 & -0.467318 \\
\hline 12 & $\mathrm{H}$ & -0.155518 & 1.095011 & -1.753806 \\
\hline 13 & $\mathrm{H}$ & -0.262866 & -2.163196 & 1.533834 \\
\hline 14 & $\mathrm{O}$ & -0.445925 & -1.271222 & 3.356450 \\
\hline
\end{tabular}

transition state of C-C cleavage of trans- $C_{1}$-formyl $c i s-N$-formyl 2-aziridinylmethyl radical

\begin{tabular}{ccccc} 
Center & Atomic & \multicolumn{3}{c}{ Coordinates (Angstroms) } \\
Number & Number & X & Y & Z \\
-1 & N & 0.000000 & 0.000000 & 0.000000 \\
2 & $\mathrm{C}$ & 0.000000 & 0.000000 & 1.417653 \\
3 & $\mathrm{C}$ & 1.396756 & 0.000000 & 0.305232 \\
4 & $\mathrm{C}$ & 2.200058 & -1.146021 & 0.270491 \\
5 & $\mathrm{H}$ & 1.769513 & -2.138358 & 0.182770 \\
6 & $\mathrm{H}$ & 3.271964 & -1.060869 & 0.403990 \\
7 & $\mathrm{H}$ & 1.866069 & 0.980689 & 0.293489 \\
8 & $\mathrm{H}$ & -0.125268 & -0.942117 & 1.946011 \\
9 & $\mathrm{C}$ & -0.207254 & 1.252633 & 2.148177 \\
10 & $\mathrm{C}$ & -0.663166 & -0.927107 & -0.800047 \\
11 & $\mathrm{O}$ & -1.709127 & -1.458361 & -0.504417 \\
12 & $\mathrm{H}$ & -0.142938 & -1.072798 & -1.764048 \\
13 & $\mathrm{H}$ & -0.222665 & 2.163850 & 1.512284 \\
14 & $\mathrm{O}$ & -0.331554 & 1.304550 & 3.359926
\end{tabular}

transition state of C-C cleavage of cis- $_{l}$-phenyl trans- $N$-formyl 2-aziridinylmethyl radical

\begin{tabular}{ccccc} 
Center & Atomic & \multicolumn{3}{c}{ Coordinates (Angstroms) } \\
Number & Number & X & Y & Z \\
- \hdashline---0000000 & & \\
1 & N & 0.000000 & 0.000000 & 0.000000 \\
2 & C & 0.000000 & 0.000000 & 1.424508
\end{tabular}




$\begin{array}{rrrrr}3 & \mathrm{C} & 1.395757 & 0.000000 & 0.313856 \\ 4 & \mathrm{C} & 2.212088 & -1.110419 & 0.073175 \\ 5 & \mathrm{H} & 1.791360 & -2.044129 & -0.282648 \\ 6 & \mathrm{H} & 3.265454 & -1.076444 & 0.327980 \\ 7 & \mathrm{H} & 1.862555 & 0.971198 & 0.493916 \\ 8 & \mathrm{H} & -0.071634 & 0.946514 & 1.962295 \\ 9 & \mathrm{C} & -0.287579 & -1.228608 & 2.162977 \\ 10 & \mathrm{C} & -0.586486 & 1.067387 & -0.724402 \\ 11 & \mathrm{C} & -1.833831 & 1.576764 & -0.340303 \\ 12 & \mathrm{C} & -2.433279 & 2.584913 & -1.093549 \\ 13 & \mathrm{C} & -1.800312 & 3.094799 & -2.229101 \\ 14 & \mathrm{C} & -0.557712 & 2.582962 & -2.609115 \\ 15 & \mathrm{C} & 0.051068 & 1.573981 & -1.864738 \\ 16 & \mathrm{H} & 1.014659 & 1.170975 & -2.163423 \\ 17 & \mathrm{H} & -0.055116 & 2.971261 & -3.490995 \\ 18 & \mathrm{H} & -2.269948 & 3.882201 & -2.811338 \\ 19 & \mathrm{H} & -3.400964 & 2.973766 & -0.788039 \\ 20 & \mathrm{H} & -2.330061 & 1.176077 & 0.539199 \\ 21 & \mathrm{H} & -0.326189 & -2.147810 & 1.541097 \\ 22 & \mathrm{O} & -0.458889 & -1.257162 & 3.370639\end{array}$

transition state of C-C cleavage of trans- $C_{1}$-phenyl $c i s-N$-formyl 2-aziridinylmethyl radical

\begin{tabular}{crrrr} 
Center & Atomic & \multicolumn{3}{c}{ Coordinates (Angstroms) } \\
Number & Number & X & Y & Z \\
- & N & 0.000000 & 0.000000 & 0.000000 \\
1 & $\mathrm{C}$ & 0.000000 & 0.000000 & 1.421299 \\
3 & $\mathrm{C}$ & 1.404797 & 0.000000 & 0.303192 \\
3 & $\mathrm{C}$ & 2.230953 & -1.120680 & 0.312109 \\
4 & $\mathrm{H}$ & 1.836871 & -2.122969 & 0.186973 \\
5 & $\mathrm{H}$ & 3.296822 & -1.002657 & 0.468835 \\
6 & $\mathrm{H}$ & 1.854754 & 0.989710 & 0.299189 \\
7 & $\mathrm{H}$ & -0.068305 & -0.943155 & 1.962542 \\
8 & $\mathrm{C}$ & -0.210720 & 1.242731 & 2.155181 \\
9 & $\mathrm{C}$ & -0.641176 & -1.021444 & -0.744819 \\
10 & $\mathrm{C}$ & -1.836762 & -1.586742 & -0.283727 \\
11 & $\mathrm{C}$ & -2.512307 & -2.523234 & -1.067178 \\
12 & $\mathrm{C}$ & -2.005260 & -2.908206 & -2.308274 \\
13 & $\mathrm{C}$ & -0.812863 & -2.340122 & -2.766046 \\
14 & $\mathrm{C}$ & -0.132707 & -1.400243 & -1.996161 \\
15 & $\mathrm{H}$ & 0.791374 & -0.957492 & -2.355351 \\
16 & $\mathrm{H}$ & -0.408287 & -2.628683 & -3.732588 \\
17 & $\mathrm{H}$ & -2.531818 & -3.640211 & -2.913602 \\
18 & $\mathrm{H}$ & -3.440612 & -2.952443 & -0.699740 \\
19 & & & &
\end{tabular}




$\begin{array}{llrrr}20 & \mathrm{H} & -2.241372 & -1.282398 & 0.677281 \\ 21 & \mathrm{O} & -0.316893 & 1.295699 & 3.372303 \\ 22 & \mathrm{H} & -0.250034 & 2.153365 & 1.519632\end{array}$

transition state of C-C cleavage of $c i s$ - $C_{1}$-methyl trans- $N$-phenyl 2-aziridinylmethyl radical

\begin{tabular}{|c|c|c|c|c|}
\hline \multirow{3}{*}{$\begin{array}{l}\text { Center } \\
\text { Number } \\
1\end{array}$} & \multirow{3}{*}{$\begin{array}{l}\text { Atomic } \\
\text { Number } \\
\mathrm{N}\end{array}$} & \multicolumn{3}{|c|}{ Coordinates (Angstroms) } \\
\hline & & $\mathrm{X}$ & $\mathrm{Y}$ & $\mathrm{Z}$ \\
\hline & & 0.000000 & 0.000000 & 0.000000 \\
\hline 2 & $\mathrm{C}$ & 0.000000 & 0.000000 & 1.424421 \\
\hline 3 & $\mathrm{C}$ & 1.401266 & 0.000000 & 0.325208 \\
\hline 4 & $\mathrm{C}$ & 2.222276 & -1.109846 & 0.116132 \\
\hline 5 & $\mathrm{H}$ & 1.806650 & -2.067337 & -0.175313 \\
\hline 6 & $\mathrm{H}$ & 3.275853 & -1.060569 & 0.369207 \\
\hline 7 & $\mathrm{H}$ & 1.868808 & 0.976853 & 0.482885 \\
\hline 8 & $\mathrm{H}$ & -0.006171 & 0.968657 & 1.932513 \\
\hline 9 & $\mathrm{C}$ & -0.417220 & -1.161162 & 2.200091 \\
\hline 10 & $\mathrm{C}$ & -0.519654 & 1.188820 & -0.661475 \\
\hline 11 & $\mathrm{H}$ & -0.218820 & 1.173429 & -1.713770 \\
\hline 12 & $\mathrm{H}$ & -1.612876 & 1.179974 & -0.604577 \\
\hline 13 & $\mathrm{H}$ & -0.153710 & 2.127315 & -0.206408 \\
\hline 14 & $\mathrm{C}$ & -0.793260 & -2.372285 & 1.588095 \\
\hline 15 & $\mathrm{C}$ & -1.175625 & -3.465712 & 2.360754 \\
\hline 16 & $\mathrm{C}$ & -1.191164 & -3.378418 & 3.755876 \\
\hline 17 & $\mathrm{C}$ & -0.822601 & -2.180182 & 4.374649 \\
\hline 18 & $\mathrm{C}$ & -0.438721 & -1.085012 & 3.606401 \\
\hline 19 & $\mathrm{H}$ & -0.151424 & -0.155284 & 4.093131 \\
\hline 20 & $\mathrm{H}$ & -0.835526 & -2.100166 & 5.458663 \\
\hline 21 & $\mathrm{H}$ & -1.490372 & -4.234098 & 4.355116 \\
\hline 22 & $\mathrm{H}$ & -1.468338 & -4.391813 & 1.872162 \\
\hline 23 & $\mathrm{H}$ & -0.794481 & -2.429821 & 0.504481 \\
\hline
\end{tabular}

transition state of C-C cleavage of trans- $C_{1}$-methyl cis- $N$-phenyl 2-aziridinylmethyl radical

\begin{tabular}{ccccc} 
Center & Atomic & \multicolumn{3}{c}{ Coordinates (Angstroms) } \\
Number & Number & X & Y & Z \\
---1 & N & 0.000000 & 0.000000 & 0.000000 \\
1 & N & 0.000000 & 0.000000 & 1.429268 \\
2 & $\mathrm{C}$ & 1.399466 & 0.000000 & 0.349509 \\
3 & $\mathrm{C}$ & 2.269938 & -1.090003 & 0.277657 \\
4 & $\mathrm{C}$ & 3.327345 & -0.943269 & 0.465467 \\
5 & $\mathrm{H}$ & 1.931453 & -2.100048 & 0.078284 \\
6 & $\mathrm{H}$ & 1.824845 & 1.000363 & 0.395336 \\
7 & $\mathrm{H}$ & & &
\end{tabular}




$\begin{array}{rrrrr}8 & \mathrm{H} & -0.049048 & -0.966221 & 1.933951 \\ 9 & \mathrm{C} & -0.339772 & 1.190806 & 2.194077 \\ 10 & \mathrm{C} & -0.559748 & -1.170379 & -0.663457 \\ 11 & \mathrm{H} & -0.346348 & -2.122131 & -0.149236 \\ 12 & \mathrm{H} & -1.646123 & -1.049003 & -0.714326 \\ 13 & \mathrm{H} & -0.160221 & -1.226707 & -1.680789 \\ 14 & \mathrm{C} & -0.613416 & 2.421197 & 1.562679 \\ 15 & \mathrm{C} & -0.913670 & 3.550914 & 2.318481 \\ 16 & \mathrm{C} & -0.946845 & 3.482723 & 3.714735 \\ 17 & \mathrm{C} & -0.678210 & 2.266935 & 4.351900 \\ 18 & \mathrm{C} & -0.375933 & 1.134549 & 3.602371 \\ 19 & \mathrm{H} & -0.165941 & 0.191488 & 4.102403 \\ 20 & \mathrm{H} & -0.706064 & 2.203242 & 5.436725 \\ 21 & \mathrm{H} & -1.182314 & 4.366839 & 4.300899 \\ 22 & \mathrm{H} & -1.129034 & 4.490893 & 1.816392 \\ 23 & \mathrm{H} & -0.601219 & 2.463013 & 0.477886\end{array}$

transition state of C-C cleavage of $c i s-C_{l}$-formyl trans- $N$-phenyl 2-aziridinylmethyl radical

\begin{tabular}{crrrr} 
Center & Atomic & \multicolumn{3}{c}{ Coordinates (Angstroms) } \\
Number & Number & X & Y & Z \\
-1 & N & 0.000000 & 0.000000 & 0.000000 \\
2 & $\mathrm{C}$ & 0.000000 & 0.000000 & 1.415982 \\
3 & $\mathrm{C}$ & 1.403468 & 0.000000 & 0.307905 \\
4 & $\mathrm{C}$ & 2.218956 & -1.118539 & 0.090318 \\
5 & $\mathrm{H}$ & 1.796870 & -2.080462 & -0.175420 \\
6 & $\mathrm{H}$ & 3.279033 & -1.063078 & 0.311422 \\
7 & $\mathrm{H}$ & 1.871665 & 0.977173 & 0.429691 \\
8 & $\mathrm{H}$ & -0.076585 & 0.971588 & 1.903643 \\
9 & $\mathrm{C}$ & -0.378628 & -1.177734 & 2.192495 \\
10 & $\mathrm{C}$ & -0.639589 & 0.944847 & -0.792167 \\
11 & $\mathrm{O}$ & -1.673491 & 1.504490 & -0.498009 \\
12 & $\mathrm{H}$ & -0.110016 & 1.094057 & -1.751968 \\
13 & $\mathrm{C}$ & -0.747409 & -2.390257 & 1.583029 \\
14 & $\mathrm{C}$ & -1.084909 & -3.495897 & 2.359698 \\
15 & $\mathrm{C}$ & -1.062348 & -3.413574 & 3.754584 \\
16 & $\mathrm{C}$ & -0.703018 & -2.211406 & 4.370383 \\
17 & $\mathrm{C}$ & -0.363715 & -1.104704 & 3.597543 \\
18 & $\mathrm{H}$ & -0.085531 & -0.170344 & 4.079818 \\
19 & $\mathrm{H}$ & -0.689710 & -2.136782 & 5.454450 \\
20 & $\mathrm{H}$ & -1.328669 & -4.277471 & 4.357331 \\
21 & $\mathrm{H}$ & -1.374784 & -4.424497 & 1.874987 \\
22 & $\mathrm{H}$ & -0.786210 & -2.446095 & 0.499656 \\
& & & &
\end{tabular}


transition state of C-C cleavage of trans- $C_{1}$-formyl $c i s-N$-phenyl 2-aziridinylmethyl radical

\begin{tabular}{|c|c|c|c|c|}
\hline \multirow{2}{*}{$\begin{array}{l}\text { Center } \\
\text { Number }\end{array}$} & \multirow{2}{*}{$\begin{array}{l}\text { Atomic } \\
\text { Number }\end{array}$} & \multicolumn{3}{|c|}{ Coordinates (Angstroms) } \\
\hline & & $\mathrm{X}$ & $\mathrm{Y}$ & $\mathrm{Z}$ \\
\hline 1 & $\mathrm{~N}$ & 0.000000 & 0.000000 & 0.000000 \\
\hline 2 & $\mathrm{C}$ & 0.000000 & 0.000000 & 1.418265 \\
\hline 3 & $\mathrm{C}$ & 1.398199 & 0.000000 & 0.317951 \\
\hline 4 & $\mathrm{C}$ & 2.217691 & -1.132195 & 0.213411 \\
\hline 5 & $\mathrm{H}$ & 3.287093 & -1.042124 & 0.363840 \\
\hline 6 & $\mathrm{H}$ & 1.801473 & -2.125449 & 0.078245 \\
\hline 7 & $\mathrm{H}$ & 1.850835 & 0.988214 & 0.346138 \\
\hline 8 & $\mathrm{H}$ & -0.098462 & -0.967217 & 1.905993 \\
\hline 9 & $\mathrm{C}$ & -0.315887 & 1.201584 & 2.182142 \\
\hline 10 & $\mathrm{C}$ & -0.648523 & -0.922737 & -0.807763 \\
\hline 11 & $\mathrm{O}$ & -1.700873 & -1.459852 & -0.535910 \\
\hline 12 & $\mathrm{H}$ & -0.109647 & -1.069774 & -1.762246 \\
\hline 13 & $\mathrm{C}$ & -0.576134 & 2.434274 & 1.553375 \\
\hline 14 & $\mathrm{C}$ & -0.844628 & 3.569418 & 2.313197 \\
\hline 15 & $\mathrm{C}$ & -0.859645 & 3.498999 & 3.709302 \\
\hline 16 & $\mathrm{C}$ & -0.606416 & 2.278822 & 4.343292 \\
\hline 17 & $\mathrm{C}$ & -0.335029 & 1.141573 & 3.589114 \\
\hline 18 & $\mathrm{H}$ & -0.138911 & 0.193589 & 4.084679 \\
\hline 19 & $\mathrm{H}$ & -0.623146 & 2.214855 & 5.427996 \\
\hline 20 & $\mathrm{H}$ & -1.072318 & 4.386510 & 4.298792 \\
\hline 21 & $\mathrm{H}$ & -1.051030 & 4.512932 & 1.814804 \\
\hline 22 & $\mathrm{H}$ & -0.582952 & 2.482243 & 0.468491 \\
\hline
\end{tabular}

transition state of C-C cleavage of $c i s$ - $C_{1}$-phenyl trans- $N$-phenyl 2-aziridinylmethyl radical

\begin{tabular}{ccccc} 
Center & Atomic & \multicolumn{3}{c}{ Coordinates (Angstroms) } \\
Number & Number & X & Y & Z \\
- & Nume & & \\
1 & $\mathrm{~N}$ & 0.000000 & 0.000000 & 0.000000 \\
2 & $\mathrm{C}$ & 0.000000 & 0.000000 & 1.421762 \\
3 & $\mathrm{C}$ & 1.401639 & 0.000000 & 0.314357 \\
4 & $\mathrm{C}$ & 2.216681 & -1.114367 & 0.101549 \\
5 & $\mathrm{H}$ & 1.795877 & -2.071398 & -0.183836 \\
6 & $\mathrm{H}$ & 3.271748 & -1.067520 & 0.348382 \\
7 & $\mathrm{H}$ & 1.867177 & 0.978533 & 0.452420 \\
8 & $\mathrm{H}$ & -0.028320 & 0.970770 & 1.921375 \\
9 & $\mathrm{C}$ & -0.402172 & -1.167332 & 2.197891 \\
10 & $\mathrm{C}$ & -0.582071 & 1.056119 & -0.736864 \\
11 & $\mathrm{C}$ & -1.827700 & 1.578063 & -0.359835 \\
12 & $\mathrm{C}$ & -2.422533 & 2.580211 & -1.124510
\end{tabular}




$\begin{array}{llrrr}13 & \mathrm{C} & -1.787819 & 3.074978 & -2.266013 \\ 14 & \mathrm{C} & -0.547266 & 2.552517 & -2.639153 \\ 15 & \mathrm{C} & 0.056849 & 1.549424 & -1.883394 \\ 16 & \mathrm{H} & 1.019343 & 1.139030 & -2.175312 \\ 17 & \mathrm{H} & -0.042483 & 2.928718 & -3.525305 \\ 18 & \mathrm{H} & -2.253793 & 3.858007 & -2.857261 \\ 19 & \mathrm{H} & -3.388629 & 2.977191 & -0.823425 \\ 20 & \mathrm{H} & -2.324927 & 1.188624 & 0.524239 \\ 21 & \mathrm{C} & -0.775979 & -2.379711 & 1.588033 \\ 22 & \mathrm{C} & -1.138372 & -3.477783 & 2.363939 \\ 23 & \mathrm{C} & -1.136034 & -3.391892 & 3.759133 \\ 24 & \mathrm{C} & -0.770455 & -2.191595 & 4.375510 \\ 25 & \mathrm{C} & -0.406401 & -1.091898 & 3.604120 \\ 26 & \mathrm{H} & -0.120746 & -0.160224 & 4.087795 \\ 27 & \mathrm{H} & -0.770486 & -2.113495 & 5.459619 \\ 28 & \mathrm{H} & -1.420404 & -4.250839 & 4.360836 \\ 29 & \mathrm{H} & -1.430308 & -4.405380 & 1.878069 \\ 30 & \mathrm{H} & -0.792596 & -2.437487 & 0.504343\end{array}$

transition state of C-C cleavage of trans- $C_{1}$-phenyl cis- $N$-phenyl 2-aziridinylmethyl radical

\begin{tabular}{|c|c|c|c|c|}
\hline \multirow{2}{*}{$\begin{array}{l}\text { Center } \\
\text { Number }\end{array}$} & \multirow{2}{*}{$\begin{array}{l}\text { Atomic } \\
\text { Number }\end{array}$} & \multicolumn{3}{|c|}{ Coordinates (Angstroms) } \\
\hline & & $\mathrm{X}$ & $\mathrm{Y}$ & $\mathrm{Z}$ \\
\hline 1 & $\mathrm{~N}$ & 0.000000 & 0.000000 & 0.000000 \\
\hline 2 & $\mathrm{C}$ & 0.000000 & 0.000000 & 1.421919 \\
\hline 3 & $\mathrm{C}$ & 1.405658 & 0.000000 & 0.313788 \\
\hline 4 & $\mathrm{C}$ & 2.246936 & -1.110095 & 0.255730 \\
\hline 5 & $\mathrm{H}$ & 1.866042 & -2.109441 & 0.077965 \\
\hline 6 & $\mathrm{H}$ & 3.309751 & -0.989257 & 0.430935 \\
\hline 7 & $\mathrm{H}$ & 1.840064 & 0.996186 & 0.349321 \\
\hline 8 & $\mathrm{C}$ & -0.330104 & 1.193819 & 2.185461 \\
\hline 9 & $\mathrm{H}$ & -0.037432 & -0.965667 & 1.925985 \\
\hline 10 & $\mathrm{C}$ & -0.636220 & -1.012435 & -0.753612 \\
\hline 11 & $\mathrm{C}$ & -1.831476 & -1.588112 & -0.300851 \\
\hline 12 & $\mathrm{C}$ & -2.502874 & -2.520078 & -1.092721 \\
\hline 13 & $\mathrm{C}$ & -1.992599 & -2.894811 & -2.335993 \\
\hline 14 & $\mathrm{C}$ & -0.801019 & -2.318631 & -2.785999 \\
\hline 15 & $\mathrm{C}$ & -0.125418 & -1.382173 & -2.008022 \\
\hline 16 & $\mathrm{H}$ & 0.798610 & -0.934447 & -2.360574 \\
\hline 17 & $\mathrm{H}$ & -0.392990 & -2.598933 & -3.753798 \\
\hline 18 & $\mathrm{H}$ & -2.515166 & -3.624398 & -2.947905 \\
\hline 19 & $\mathrm{H}$ & -3.430784 & -2.955103 & -0.730339 \\
\hline 20 & $\mathrm{H}$ & -2.237349 & -1.291945 & 0.662179 \\
\hline 21 & $\mathrm{C}$ & -0.617197 & 2.421518 & 1.554882 \\
\hline
\end{tabular}




$\begin{array}{lllll}22 & \mathrm{C} & -0.906459 & 3.552540 & 2.312674 \\ 23 & \mathrm{C} & -0.915622 & 3.487430 & 3.709489 \\ 24 & \mathrm{C} & -0.633698 & 2.274238 & 4.345754 \\ 25 & \mathrm{C} & -0.341787 & 1.140503 & 3.594430 \\ 26 & \mathrm{H} & -0.121161 & 0.199150 & 4.092947 \\ 27 & \mathrm{H} & -0.643016 & 2.213699 & 5.430961 \\ 28 & \mathrm{H} & -1.143261 & 4.372574 & 4.297077 \\ 29 & \mathrm{H} & -1.132617 & 4.490596 & 1.812046 \\ 30 & \mathrm{H} & -0.623803 & 2.462893 & 0.469918\end{array}$

\begin{tabular}{|c|c|c|c|c|}
\hline \multirow{2}{*}{$\begin{array}{l}\text { Center } \\
\text { Number }\end{array}$} & \multirow{2}{*}{$\begin{array}{l}\text { Atomic } \\
\text { Number }\end{array}$} & \multicolumn{3}{|c|}{ Coordinates (Angstroms) } \\
\hline & & $\mathrm{X}$ & Y & $\mathrm{Z}$ \\
\hline 1 & $\mathrm{C}$ & 0.000000 & 0.000000 & 0.000000 \\
\hline 2 & $\mathrm{C}$ & 0.000000 & 0.000000 & 1.495697 \\
\hline 3 & $\mathrm{C}$ & 1.338781 & 0.000000 & 0.748097 \\
\hline 4 & $\mathrm{C}$ & 2.198205 & -1.177137 & 0.748199 \\
\hline 5 & $\mathrm{H}$ & 1.770800 & -2.175460 & 0.750027 \\
\hline 6 & $\mathrm{H}$ & 3.278048 & -1.085116 & 0.744443 \\
\hline 7 & $\mathrm{H}$ & 1.859817 & 0.954989 & 0.747897 \\
\hline 8 & $\mathrm{H}$ & -0.274878 & -0.915983 & 2.011838 \\
\hline 9 & $\mathrm{H}$ & -0.281626 & 0.909956 & 2.019426 \\
\hline 10 & $\mathrm{H}$ & -0.281431 & 0.909999 & -0.523763 \\
\hline 11 & $\mathrm{H}$ & -0.274739 & -0.915986 & -0.516186 \\
\hline
\end{tabular}

cis- $C_{1}$-formyl cyclopropylcarbinyl radical

\begin{tabular}{ccrrr} 
Center & Atomic & \multicolumn{3}{c}{ Coordinates (Angstroms) } \\
Number & Number & X & Y & Z \\
-1 & C & 0.000000 & 0.000000 & 0.000000 \\
2 & C & 0.000000 & 0.000000 & 1.503429 \\
3 & $\mathrm{C}$ & 1.357451 & 0.000000 & 0.640621 \\
4 & $\mathrm{C}$ & 2.242074 & -1.144546 & 0.600285 \\
5 & $\mathrm{H}$ & 1.848353 & -2.144983 & 0.470128 \\
6 & $\mathrm{H}$ & 3.295139 & -1.025043 & 0.829331 \\
7 & $\mathrm{H}$ & 1.845513 & 0.969712 & 0.707522 \\
8 & $\mathrm{H}$ & -0.193022 & 0.935851 & 2.021473 \\
9 & $\mathrm{C}$ & -0.372156 & -1.225448 & 2.234942 \\
10 & $\mathrm{H}$ & -0.317272 & -0.930437 & -0.463508 \\
11 & $\mathrm{H}$ & -0.331837 & 0.904633 & -0.502375 \\
12 & $\mathrm{O}$ & -0.356615 & -2.348203 & 1.760862 \\
13 & $\mathrm{H}$ & -0.665623 & -1.066343 & 3.295535
\end{tabular}


trans- $C_{1}$-formyl cyclopropylcarbinyl radical

\begin{tabular}{|c|c|c|c|c|}
\hline \multirow{2}{*}{$\begin{array}{l}\text { Center } \\
\text { Number }\end{array}$} & \multirow{2}{*}{$\begin{array}{l}\text { Atomic } \\
\text { Number }\end{array}$} & \multicolumn{3}{|c|}{ Coordinates (Angstroms) } \\
\hline & & $X$ & $\mathrm{Y}$ & $\mathrm{Z}$ \\
\hline 1 & $\mathrm{C}$ & 0.000000 & 0.000000 & 0.000000 \\
\hline 2 & $\mathrm{C}$ & 0.000000 & 0.000000 & 1.502694 \\
\hline 3 & $\mathrm{C}$ & 1.355418 & 0.000000 & 0.636309 \\
\hline 4 & $\mathrm{C}$ & 2.205327 & -1.165897 & 0.662696 \\
\hline 5 & $\mathrm{H}$ & 1.802136 & -2.155811 & 0.471740 \\
\hline 6 & $\mathrm{H}$ & 3.244005 & -1.089087 & 0.963498 \\
\hline 7 & $\mathrm{H}$ & 1.841791 & 0.970657 & 0.691024 \\
\hline 8 & $\mathrm{H}$ & -0.212586 & -0.926563 & 2.027041 \\
\hline 9 & $\mathrm{C}$ & -0.276471 & 1.256207 & 2.222834 \\
\hline 10 & $\mathrm{H}$ & -0.323146 & 0.926232 & -0.467023 \\
\hline 11 & $\mathrm{H}$ & -0.323425 & -0.911756 & -0.495438 \\
\hline 12 & $\mathrm{O}$ & -0.165365 & 2.365538 & 1.727820 \\
\hline 13 & $\mathrm{H}$ & -0.579634 & 1.137615 & 3.285395 \\
\hline
\end{tabular}

$c i s-C_{1}$-phenyl cyclopropylcarbinyl radical

\begin{tabular}{crrrr} 
Center & Atomic & \multicolumn{3}{c}{ Coordinates (Angstroms) } \\
Number & Number & X & Y & $\mathrm{Z}$ \\
- & Nume & & \\
1 & $\mathrm{C}$ & 0.000000 & 0.000000 & 0.000000 \\
2 & $\mathrm{C}$ & 0.000000 & 0.000000 & 1.495638 \\
3 & $\mathrm{C}$ & 1.349717 & 0.000000 & 0.724076 \\
4 & $\mathrm{C}$ & 2.225207 & -1.161086 & 0.699406 \\
5 & $\mathrm{H}$ & 1.822326 & -2.167690 & 0.723398 \\
6 & $\mathrm{H}$ & 3.302348 & -1.041548 & 0.687705 \\
7 & $\mathrm{H}$ & 1.859124 & 0.961168 & 0.736940 \\
8 & $\mathrm{H}$ & -0.212944 & 0.960923 & 1.963787 \\
9 & $\mathrm{C}$ & -0.441685 & -1.176768 & 2.308315 \\
10 & $\mathrm{H}$ & -0.264978 & -0.920206 & -0.513545 \\
11 & $\mathrm{H}$ & -0.296244 & 0.904594 & -0.525025 \\
12 & $\mathrm{C}$ & -1.403940 & -2.082736 & 1.844655 \\
13 & $\mathrm{C}$ & -1.815628 & -3.159301 & 2.633888 \\
14 & $\mathrm{C}$ & -1.271932 & -3.345421 & 3.904654 \\
15 & $\mathrm{C}$ & -0.313743 & -2.446736 & 4.381453 \\
16 & $\mathrm{C}$ & 0.096421 & -1.375794 & 3.589879 \\
17 & $\mathrm{H}$ & 0.848222 & -0.683654 & 3.961676 \\
18 & $\mathrm{H}$ & 0.117151 & -2.583757 & 5.370059 \\
19 & $\mathrm{H}$ & -1.591124 & -4.182399 & 4.520083 \\
20 & $\mathrm{H}$ & -2.565304 & -3.849311 & 2.254866
\end{tabular}




\begin{tabular}{|c|c|c|c|c|}
\hline 21 & $\mathrm{H}$ & -1.842966 & -1.937991 & 0.861292 \\
\hline$n s-C_{l}$ & cyclo & lcarbiny & ical & \\
\hline Center & Atomic & Coor & inates (Angs & oms) \\
\hline Number & Number & $X$ & Y & $\mathrm{Z}$ \\
\hline 1 & $\mathrm{C}$ & 0.000000 & 0.000000 & 0.000000 \\
\hline 2 & $\mathrm{C}$ & 0.000000 & 0.000000 & 1.500073 \\
\hline 3 & $\mathrm{C}$ & 1.349222 & 0.000000 & 0.682517 \\
\hline 4 & $\mathrm{C}$ & 2.211383 & -1.166195 & 0.684276 \\
\hline 5 & $\mathrm{H}$ & 1.803553 & -2.158569 & 0.516342 \\
\hline 6 & $\mathrm{H}$ & 3.253899 & -1.088087 & 0.971528 \\
\hline 7 & $\mathrm{H}$ & 1.853847 & 0.963225 & 0.723676 \\
\hline 8 & $\mathrm{H}$ & -0.218209 & -0.956125 & 1.969150 \\
\hline 9 & $\mathrm{C}$ & -0.377286 & 1.178145 & 2.322112 \\
\hline 10 & $\mathrm{H}$ & -0.302447 & -0.916446 & -0.501353 \\
\hline 11 & $\mathrm{H}$ & -0.312014 & 0.906102 & -0.513523 \\
\hline 12 & $\mathrm{C}$ & -0.308307 & 2.495923 & 1.839248 \\
\hline 13 & $\mathrm{C}$ & -0.655608 & 3.575722 & 2.649707 \\
\hline 14 & $\mathrm{C}$ & -1.083581 & 3.365964 & 3.962276 \\
\hline 15 & $\mathrm{C}$ & -1.158524 & 2.062461 & 4.455696 \\
\hline 16 & $\mathrm{C}$ & -0.807627 & 0.983974 & 3.644859 \\
\hline 17 & $\mathrm{H}$ & -0.869078 & -0.028113 & 4.038638 \\
\hline 18 & $\mathrm{H}$ & 0.019020 & 2.685403 & 0.820028 \\
\hline 19 & $\mathrm{H}$ & -0.594106 & 4.585654 & 2.252126 \\
\hline 20 & $\mathrm{H}$ & -1.356222 & 4.208540 & 4.591847 \\
\hline 21 & $\mathrm{H}$ & -1.491491 & 1.882809 & 5.474806 \\
\hline
\end{tabular}

oxiranylcarbinyl radical

\begin{tabular}{ccccc} 
Center & Atomic & \multicolumn{3}{c}{ Coordinates (Angstroms) } \\
Number & Number & X & Y & Z \\
- & O & 0.000000 & 0.000000 & 0.000000 \\
1 & O & 0.000000 & 0.000000 & 1.418023 \\
2 & $\mathrm{C}$ & 1.294047 & 0.000000 & 0.680831 \\
3 & $\mathrm{C}$ & 2.112613 & -1.190527 & 0.539201 \\
4 & $\mathrm{C}$ & 1.644515 & -2.166592 & 0.473054 \\
5 & $\mathrm{H}$ & 3.192711 & -1.121486 & 0.484886 \\
6 & $\mathrm{H}$ & 1.810839 & 0.958136 & 0.616203 \\
7 & $\mathrm{H}$ & -0.341934 & -0.920908 & 1.893052 \\
8 & $\mathrm{H}$ & -0.335485 & 0.922088 & 1.897530 \\
9 & $\mathrm{H}$ & &
\end{tabular}

cis- $C_{1}$-formyl oxiranylcarbinyl radical 


\begin{tabular}{crrrr} 
Center & Atomic & \multicolumn{3}{c}{ Coordinates (Angstroms) } \\
Number & Number & X & Y & Z \\
--1 & O & 0.000000 & 0.000000 & 0.000000 \\
2 & $\mathrm{C}$ & 0.000000 & 0.000000 & 1.423729 \\
3 & $\mathrm{C}$ & 1.293574 & 0.000000 & 0.624447 \\
4 & $\mathrm{C}$ & 2.153643 & -1.151763 & 0.427659 \\
5 & $\mathrm{H}$ & 1.740295 & -2.119861 & 0.171384 \\
6 & $\mathrm{H}$ & 3.225999 & -1.052147 & 0.545713 \\
7 & $\mathrm{H}$ & 1.787574 & 0.973644 & 0.614067 \\
8 & $\mathrm{H}$ & -0.292051 & 0.932634 & 1.908949 \\
9 & $\mathrm{C}$ & -0.484386 & -1.241931 & 2.093147 \\
10 & $\mathrm{H}$ & -0.435479 & -2.160709 & 1.471338 \\
11 & $\mathrm{O}$ & -0.902232 & -1.253120 & 3.230711
\end{tabular}

trans- $C_{1}$-formyl oxiranylcarbinyl radical

\begin{tabular}{ccccc} 
Center & Atomic & \multicolumn{3}{c}{ Coordinates (Angstroms) } \\
Number & Number & X & Y & Z \\
---1 & O & 0.000000 & 0.000000 & 0.000000 \\
1 & C & 0.000000 & 0.000000 & 1.422575 \\
2 & $\mathrm{C}$ & 1.294038 & 0.000000 & 0.629999 \\
3 & $\mathrm{C}$ & 2.108557 & -1.191716 & 0.518949 \\
4 & $\mathrm{H}$ & 1.640484 & -2.161497 & 0.394084 \\
5 & $\mathrm{H}$ & 3.186229 & -1.130875 & 0.611844 \\
6 & $\mathrm{H}$ & 1.810643 & 0.959163 & 0.567026 \\
7 & $\mathrm{H}$ & -0.309448 & -0.923625 & 1.911021 \\
8 & $\mathrm{C}$ & -0.417708 & 1.269422 & 2.084715 \\
9 & $\mathrm{H}$ & -0.312442 & 2.173122 & 1.444292 \\
10 & $\mathrm{O}$ & -0.818582 & 1.325083 & 3.226933 \\
11 & & &
\end{tabular}

cis- $C_{1}$-phenyl oxiranylcarbinyl radical

\begin{tabular}{ccccc} 
Center & Atomic & \multicolumn{3}{c}{ Coordinates (Angstroms) } \\
Number & Number & X & Y & Z \\
- & X & & \\
1 & O & 0.000000 & 0.000000 & 0.000000 \\
2 & C & 0.000000 & 0.000000 & 1.417374 \\
3 & C & 1.306650 & 0.000000 & 0.660065 \\
4 & C & 2.155388 & -1.161142 & 0.505071 \\
5 & H & 1.740906 & -2.161588 & 0.519476 \\
6 & H & 3.228176 & -1.040462 & 0.410371 \\
7 & H & 1.803362 & 0.969175 & 0.598912
\end{tabular}




$\begin{array}{rrrrr}8 & \mathrm{H} & -0.268140 & 0.960889 & 1.867493 \\ 9 & \mathrm{C} & -0.554026 & -1.188978 & 2.126501 \\ 10 & \mathrm{C} & -0.977403 & -2.324102 & 1.424061 \\ 11 & \mathrm{C} & -1.489817 & -3.425628 & 2.110423 \\ 12 & \mathrm{C} & -1.585216 & -3.405430 & 3.503152 \\ 13 & \mathrm{C} & -1.172239 & -2.272578 & 4.208541 \\ 14 & \mathrm{C} & -0.664652 & -1.169671 & 3.523398 \\ 15 & \mathrm{H} & -0.350857 & -0.286057 & 4.075410 \\ 16 & \mathrm{H} & -1.250247 & -2.246125 & 5.292242 \\ 17 & \mathrm{H} & -1.985342 & -4.263788 & 4.036128 \\ 18 & \mathrm{H} & -1.820662 & -4.299515 & 1.555172 \\ 19 & \mathrm{H} & -0.916174 & -2.323143 & 0.340409\end{array}$

trans- $C_{1}$-phenyl oxiranylcarbinyl radical

\begin{tabular}{ccccc} 
Center & Atomic & \multicolumn{3}{c}{ Coordinates (Angstroms) } \\
Number & Number & X & Y & Z \\
--1 & O & 0.000000 & 0.000000 & 0.000000 \\
1 & C & 0.000000 & 0.000000 & 1.420573 \\
2 & $\mathrm{C}$ & 1.300265 & 0.000000 & 0.649025 \\
3 & $\mathrm{C}$ & 2.123512 & -1.180635 & 0.497660 \\
4 & $\mathrm{H}$ & 1.663289 & -2.153196 & 0.362381 \\
5 & $\mathrm{H}$ & 3.203127 & -1.113813 & 0.564049 \\
6 & $\mathrm{H}$ & 1.805425 & 0.965766 & 0.607142 \\
7 & $\mathrm{H}$ & -0.288216 & -0.952411 & 1.871006 \\
8 & $\mathrm{C}$ & -0.490010 & 1.215423 & 2.126934 \\
9 & $\mathrm{C}$ & -0.687979 & 2.419101 & 1.437587 \\
10 & $\mathrm{C}$ & -1.122043 & 3.555380 & 2.119157 \\
11 & $\mathrm{C}$ & -1.362895 & 3.503148 & 3.494173 \\
12 & $\mathrm{C}$ & -1.173452 & 2.304256 & 4.185015 \\
13 & $\mathrm{C}$ & -0.742943 & 1.165962 & 3.504263 \\
14 & $\mathrm{H}$ & -0.602513 & 0.231485 & 4.043543 \\
15 & $\mathrm{H}$ & -1.366327 & 2.253750 & 5.253397 \\
16 & $\mathrm{H}$ & -1.702727 & 4.389470 & 4.023168 \\
17 & $\mathrm{H}$ & -1.278885 & 4.482946 & 1.574674 \\
18 & $\mathrm{H}$ & -0.518030 & 2.444585 & 0.365451 \\
19 & & &
\end{tabular}

transition state of $\mathrm{C}-\mathrm{N}$ cleavage of cyclopropylcarbinyl radical

\begin{tabular}{ccccc} 
Center & Atomic & \multicolumn{3}{c}{ Coordinates (Angstroms) } \\
Number & Number & X & Y & Z \\
--------1 & & \\
1 & C & 0.000000 & 0.000000 & 0.000000 \\
2 & C & 0.000000 & 0.000000 & 1.482387
\end{tabular}




$\begin{array}{rrrrr}3 & \mathrm{C} & 1.473560 & 0.000000 & 1.224361 \\ 4 & \mathrm{C} & 2.293474 & -1.113431 & 1.295154 \\ 5 & \mathrm{H} & 1.895710 & -2.095515 & 1.536986 \\ 6 & \mathrm{H} & 3.347204 & -1.051789 & 1.043802 \\ 7 & \mathrm{H} & 1.929435 & 0.974686 & 1.069926 \\ 8 & \mathrm{H} & -0.377515 & -0.908475 & 1.955600 \\ 9 & \mathrm{H} & -0.397388 & 0.892866 & 1.968700 \\ 10 & \mathrm{H} & -0.051587 & -0.932637 & -0.547004 \\ 11 & \mathrm{H} & -0.043477 & 0.926391 & -0.560621\end{array}$

transition state of C-N cleavage of $\mathrm{cis}$ - $C_{1}$-formyl cyclopropylcarbinyl radical

\begin{tabular}{ccccc} 
Center & Atomic & \multicolumn{3}{c}{ Coordinates (Angstroms) } \\
Number & Number & X & Y & Z \\
-1 & $\mathrm{C}$ & 0.000000 & 0.000000 & 0.000000 \\
2 & $\mathrm{C}$ & 0.000000 & 0.000000 & 1.477656 \\
3 & $\mathrm{C}$ & 1.498294 & 0.000000 & 1.243404 \\
4 & $\mathrm{C}$ & 2.330680 & -1.098727 & 1.272408 \\
5 & $\mathrm{H}$ & 1.953770 & -2.103694 & 1.431011 \\
6 & $\mathrm{H}$ & 3.386884 & -0.988353 & 1.051672 \\
7 & $\mathrm{H}$ & 1.934240 & 0.989813 & 1.140086 \\
8 & $\mathrm{H}$ & -0.349265 & 0.915869 & 1.962382 \\
9 & $\mathrm{C}$ & -0.518875 & -1.221022 & 2.191498 \\
10 & $\mathrm{H}$ & -0.060090 & -0.944834 & -0.523212 \\
11 & $\mathrm{H}$ & -0.050154 & 0.927572 & -0.555753 \\
12 & $\mathrm{O}$ & -0.762350 & -2.278819 & 1.652750 \\
13 & $\mathrm{H}$ & -0.626831 & -1.103556 & 3.292190
\end{tabular}

transition state of C-N cleavage of trans- $C_{1}$-formyl cyclopropylcarbinyl radical

\begin{tabular}{ccccc} 
Center & Atomic & \multicolumn{3}{c}{ Coordinates (Angstroms) } \\
Number & Number & X & Y & Z \\
-1 & $\mathrm{C}$ & 0.000000 & 0.000000 & 0.000000 \\
2 & $\mathrm{C}$ & 0.000000 & 0.000000 & 1.476330 \\
3 & $\mathrm{C}$ & 1.497335 & 0.000000 & 1.252311 \\
4 & $\mathrm{C}$ & 2.309295 & -1.104360 & 1.374016 \\
5 & $\mathrm{H}$ & 1.910112 & -2.082786 & 1.625924 \\
6 & $\mathrm{H}$ & 3.371647 & -1.038063 & 1.164001 \\
7 & $\mathrm{H}$ & 1.933400 & 0.978889 & 1.074159 \\
8 & $\mathrm{H}$ & -0.351940 & -0.913392 & 1.962141 \\
9 & $\mathrm{C}$ & -0.477406 & 1.243007 & 2.181063 \\
10 & $\mathrm{H}$ & -0.041358 & -0.934536 & -0.542763 \\
11 & $\mathrm{H}$ & -0.048321 & 0.939266 & -0.535891
\end{tabular}




$\begin{array}{lllll}12 & \mathrm{O} & -0.637380 & 2.311417 & 1.630730 \\ 13 & \mathrm{H} & -0.626650 & 1.135096 & 3.277349\end{array}$

transition state of C-N cleavage of $c i s-C_{l}$-phenyl cyclopropylcarbinyl radical

\begin{tabular}{|c|c|c|c|c|}
\hline \multirow{2}{*}{$\begin{array}{l}\text { Center } \\
\text { Number }\end{array}$} & \multirow{2}{*}{$\begin{array}{l}\text { Atomic } \\
\text { Number }\end{array}$} & \multicolumn{3}{|c|}{ Coordinates (Angstroms) } \\
\hline & & $X$ & Y & $\mathrm{Z}$ \\
\hline 1 & $\mathrm{C}$ & 0.000000 & 0.000000 & 0.000000 \\
\hline 2 & $\mathrm{C}$ & 0.000000 & 0.000000 & 1.484657 \\
\hline 3 & $\mathrm{C}$ & 1.485974 & 0.000000 & 1.235777 \\
\hline 4 & $\mathrm{C}$ & 2.320142 & -1.100662 & 1.267195 \\
\hline 5 & $\mathrm{H}$ & 1.946158 & -2.095930 & 1.484163 \\
\hline 6 & $\mathrm{H}$ & 3.372429 & -1.003083 & 1.021140 \\
\hline 7 & $\mathrm{H}$ & 1.929482 & 0.983627 & 1.103693 \\
\hline 8 & $\mathrm{H}$ & -0.331915 & 0.955587 & 1.904703 \\
\hline 9 & $\mathrm{C}$ & -0.625026 & -1.156653 & 2.230000 \\
\hline 10 & $\mathrm{H}$ & -0.002748 & -0.937099 & -0.542019 \\
\hline 11 & $\mathrm{H}$ & -0.057543 & 0.923885 & -0.562819 \\
\hline 12 & $\mathrm{C}$ & -1.692812 & -1.879759 & 1.686708 \\
\hline 13 & $\mathrm{C}$ & -2.299058 & -2.910645 & 2.408143 \\
\hline 14 & $\mathrm{C}$ & -1.842565 & -3.234163 & 3.685901 \\
\hline 15 & $\mathrm{C}$ & -0.777244 & -2.518401 & 4.238476 \\
\hline 16 & $\mathrm{C}$ & -0.174880 & -1.489414 & 3.516094 \\
\hline 17 & $\mathrm{H}$ & 0.659506 & -0.940962 & 3.945751 \\
\hline 18 & $\mathrm{H}$ & -0.413357 & -2.764209 & 5.232896 \\
\hline 19 & $\mathrm{H}$ & -2.310967 & -4.038099 & 4.247601 \\
\hline 20 & $\mathrm{H}$ & -3.128196 & -3.460197 & 1.969668 \\
\hline 21 & $\mathrm{H}$ & -2.054393 & -1.626470 & 0.693689 \\
\hline
\end{tabular}

transition state of C-N cleavage of trans- $C_{1}$-phenyl cyclopropylcarbinyl radical

\begin{tabular}{ccccc}
$\begin{array}{c}\text { Center } \\
\text { Number }\end{array}$ & $\begin{array}{c}\text { Atomic } \\
\text { Number }\end{array}$ & \multicolumn{4}{c}{ Coordinates (Angstroms) } \\
- \hdashline-1 & X & Z \\
1 & $\mathrm{C}$ & 0.000000 & 0.000000 & 0.000000 \\
2 & $\mathrm{C}$ & 0.000000 & 0.000000 & 1.483554 \\
3 & $\mathrm{C}$ & 1.477871 & 0.000000 & 1.230618 \\
4 & $\mathrm{C}$ & 2.302616 & -1.106567 & 1.321771 \\
5 & $\mathrm{H}$ & 1.910306 & -2.087068 & 1.578132 \\
6 & $\mathrm{H}$ & 3.357168 & -1.041663 & 1.074859 \\
7 & $\mathrm{H}$ & 1.922145 & 0.978225 & 1.063563 \\
8 & $\mathrm{H}$ & -0.331785 & -0.956567 & 1.902471 \\
9 & $\mathrm{C}$ & -0.601731 & 1.169837 & 2.226099 \\
10 & $\mathrm{H}$ & -0.063333 & -0.929717 & -0.550580
\end{tabular}




$\begin{array}{llrrr}11 & \mathrm{H} & -0.007761 & 0.932230 & -0.553009 \\ 12 & \mathrm{C} & -1.767339 & 1.792285 & 1.763771 \\ 13 & \mathrm{C} & -2.341920 & 2.847513 & 2.474195 \\ 14 & \mathrm{C} & -1.755039 & 3.296798 & 3.658267 \\ 15 & \mathrm{C} & -0.591204 & 2.683305 & 4.127311 \\ 16 & \mathrm{C} & -0.020089 & 1.628299 & 3.415639 \\ 17 & \mathrm{H} & 0.888697 & 1.154601 & 3.779191 \\ 18 & \mathrm{H} & -0.125897 & 3.028852 & 5.046975 \\ 19 & \mathrm{H} & -2.199085 & 4.120967 & 4.210330 \\ 20 & \mathrm{H} & -3.246657 & 3.320528 & 2.100712 \\ 21 & \mathrm{H} & -2.223106 & 1.444760 & 0.839827\end{array}$

transition state of C-N cleavage of oxiranylcarbinyl radical

\begin{tabular}{ccccc} 
Center & Atomic & \multicolumn{3}{c}{ Coordinates (Angstroms) } \\
Number & Number & X & Y & Z \\
--1 & O & 0.000000 & 0.000000 & 0.000000 \\
1 & C & 0.000000 & 0.000000 & 1.386608 \\
2 & $\mathrm{C}$ & 1.441091 & 0.000000 & 1.022125 \\
3 & $\mathrm{C}$ & 2.191614 & -1.157717 & 0.886279 \\
4 & $\mathrm{H}$ & 1.738164 & -2.134226 & 1.023849 \\
5 & $\mathrm{H}$ & 3.234936 & -1.121345 & 0.590758 \\
6 & $\mathrm{H}$ & 1.903843 & 0.970091 & 0.877580 \\
7 & $\mathrm{H}$ & -0.386583 & -0.905498 & 1.874003 \\
8 & $\mathrm{H}$ & -0.369257 & 0.914403 & 1.869908 \\
9 & & & &
\end{tabular}

transition state of C-N cleavage of $c i s-C_{l}$-formyl oxiranylcarbinyl radical

\begin{tabular}{ccccc} 
Center & Atomic & \multicolumn{3}{c}{ Coordinates (Angstroms) } \\
Number & Number & X & Y & Z \\
----------1 & & \\
1 & O & 0.000000 & 0.000000 & 0.000000 \\
2 & $\mathrm{C}$ & 0.000000 & 0.000000 & 1.384876 \\
3 & $\mathrm{C}$ & 1.456747 & 0.000000 & 1.029921 \\
4 & $\mathrm{C}$ & 2.240323 & -1.127560 & 0.865289 \\
5 & $\mathrm{H}$ & 1.838351 & -2.129422 & 0.964098 \\
6 & $\mathrm{H}$ & 3.285584 & -1.033362 & 0.591010 \\
7 & $\mathrm{H}$ & 1.894281 & 0.985322 & 0.913238 \\
8 & $\mathrm{H}$ & -0.344347 & 0.917495 & 1.880391 \\
9 & $\mathrm{C}$ & -0.583715 & -1.231337 & 2.053568 \\
10 & $\mathrm{O}$ & -1.004366 & -1.228183 & 3.187643 \\
11 & $\mathrm{H}$ & -0.609596 & -2.138872 & 1.411832
\end{tabular}

transition state of C-N cleavage of trans- $C_{1}$-formyl oxiranylcarbinyl radical 


\begin{tabular}{ccccc} 
Center & Atomic & \multicolumn{3}{c}{ Coordinates (Angstroms) } \\
Number & Number & X & Y & Z \\
-1 & O & 0.000000 & 0.000000 & 0.000000 \\
2 & C & 0.000000 & 0.000000 & 1.384974 \\
3 & $\mathrm{C}$ & 1.455246 & 0.000000 & 1.037319 \\
4 & $\mathrm{C}$ & 2.201144 & -1.157573 & 0.935276 \\
5 & $\mathrm{H}$ & 1.746346 & -2.131972 & 1.079567 \\
6 & $\mathrm{H}$ & 3.252138 & -1.121857 & 0.668441 \\
7 & $\mathrm{H}$ & 1.915770 & 0.969846 & 0.881620 \\
8 & $\mathrm{H}$ & -0.387186 & -0.895239 & 1.887756 \\
9 & $\mathrm{C}$ & -0.497807 & 1.277876 & 2.035390 \\
10 & $\mathrm{H}$ & -0.316744 & 2.192627 & 1.428168 \\
11 & $\mathrm{O}$ & -1.043345 & 1.309815 & 3.113279 \\
--------- & &
\end{tabular}

transition state of C-N cleavage of $c i s-C_{l}$-phenyl oxiranylcarbinyl radical

\begin{tabular}{ccccc} 
Center & Atomic & \multicolumn{3}{c}{ Coordinates (Angstroms) } \\
Number & Number & X & Y & Z \\
- & & 0.000000 & 0.000000 & 0.000000 \\
1 & $\mathrm{O}$ & 0.000000 & 0.000000 & 1.385421 \\
2 & $\mathrm{C}$ & 1.459375 & 0.000000 & 1.019097 \\
3 & $\mathrm{C}$ & 2.233982 & -1.137060 & 0.889610 \\
4 & $\mathrm{C}$ & 1.816685 & -2.123981 & 1.056100 \\
5 & $\mathrm{H}$ & 1.901872 & 0.978916 & 0.869009 \\
6 & $\mathrm{H}$ & -0.312795 & 0.951765 & 1.841440 \\
7 & $\mathrm{H}$ & -0.613182 & -1.182443 & 2.090309 \\
8 & $\mathrm{H}$ & -1.016821 & -2.311437 & 1.369932 \\
9 & $\mathrm{C}$ & -1.580605 & -3.403628 & 2.032198 \\
10 & $\mathrm{C}$ & -1.743697 & -3.376921 & 3.418269 \\
11 & $\mathrm{C}$ & -1.347015 & -2.249054 & 4.141639 \\
12 & $\mathrm{C}$ & -0.789431 & -1.156313 & 3.479270 \\
13 & $\mathrm{C}$ & -0.488716 & -0.275803 & 4.044043 \\
14 & $\mathrm{C}$ & -1.477980 & -2.219167 & 5.220198 \\
15 & $\mathrm{H}$ & -2.183660 & -4.227008 & 3.932988 \\
16 & $\mathrm{H}$ & -1.897088 & -4.274473 & 1.463825 \\
17 & $\mathrm{H}$ & -0.895781 & -2.311047 & 0.291248 \\
18 & $\mathrm{H}$ & & \\
19 & $\mathrm{H}$ & & &
\end{tabular}

transition state of C-N cleavage of trans- $C_{1}$-phenyl oxiranylcarbinyl radical

\begin{tabular}{llccc} 
Center & Atomic & \multicolumn{3}{c}{ Coordinates (Angstroms) } \\
Number & Number & X & Y & Z
\end{tabular}




$\begin{array}{rlrrr}1 & \mathrm{O} & 0.000000 & 0.000000 & 0.000000 \\ 2 & \mathrm{C} & 0.000000 & 0.000000 & 1.385401 \\ 3 & \mathrm{C} & 1.459782 & 0.000000 & 1.035881 \\ 4 & \mathrm{C} & 2.217482 & -1.148017 & 0.917403 \\ 5 & \mathrm{H} & 1.770494 & -2.129107 & 1.043804 \\ 6 & \mathrm{H} & 3.270403 & -1.101993 & 0.658921 \\ 7 & \mathrm{H} & 1.908169 & 0.978319 & 0.901618 \\ 8 & \mathrm{H} & -0.346185 & -0.936812 & 1.847876 \\ 9 & \mathrm{C} & -0.550105 & 1.215609 & 2.088233 \\ 10 & \mathrm{C} & -0.764739 & 2.405251 & 1.384273 \\ 11 & \mathrm{C} & -1.250675 & 3.533802 & 2.045224 \\ 12 & \mathrm{C} & -1.525152 & 3.483212 & 3.413645 \\ 13 & \mathrm{C} & -1.317653 & 2.295633 & 4.118976 \\ 14 & \mathrm{C} & -0.835959 & 1.166081 & 3.457281 \\ 15 & \mathrm{H} & -0.682269 & 0.239354 & 4.006815 \\ 16 & \mathrm{H} & -1.536495 & 2.247345 & 5.182509 \\ 17 & \mathrm{H} & -1.905392 & 4.362365 & 3.927093 \\ 18 & \mathrm{H} & -1.420779 & 4.452783 & 1.490155 \\ 19 & \mathrm{H} & -0.562227 & 2.425502 & 0.317717\end{array}$

transition state of C-C cleavage of cyclopropylcarbinyl radical

\begin{tabular}{ccccc} 
Center & Atomic & \multicolumn{3}{c}{ Coordinates (Angstroms) } \\
Number & Number & X & Y & Z \\
----------1 & \\
1 & $\mathrm{C}$ & 0.000000 & 0.000000 & 0.000000 \\
2 & $\mathrm{C}$ & 0.000000 & 0.000000 & 1.482435 \\
3 & $\mathrm{C}$ & 1.473685 & 0.000000 & 1.224732 \\
4 & $\mathrm{C}$ & 2.293496 & 1.113441 & 1.295115 \\
5 & $\mathrm{H}$ & 1.895641 & 2.095662 & 1.536234 \\
6 & $\mathrm{H}$ & 3.347423 & 1.051587 & 1.044645 \\
7 & $\mathrm{H}$ & 1.929504 & -0.974722 & 1.070378 \\
8 & $\mathrm{H}$ & -0.397121 & -0.893103 & 1.968549 \\
9 & $\mathrm{H}$ & -0.377778 & 0.908246 & 1.955852 \\
10 & $\mathrm{H}$ & -0.051979 & 0.932630 & -0.546991 \\
11 & $\mathrm{H}$ & -0.042973 & -0.926361 & -0.560699
\end{tabular}

transition state of C-C cleavage of $c i s-C_{1}$-formyl cyclopropylcarbinyl radical

\begin{tabular}{ccccc} 
Center & Atomic & \multicolumn{3}{c}{ Coordinates (Angstroms) } \\
Number & Number & X & Y & Z \\
- \hdashline---100000000 & 0.000000 & 0.000000 \\
1 & C & 0.000000 & 0.000000 & 1.489035
\end{tabular}




$\begin{array}{rrrrr}3 & \mathrm{C} & 1.437253 & 0.000000 & 0.403425 \\ 4 & \mathrm{C} & 2.277247 & -1.124563 & 0.357018 \\ 5 & \mathrm{H} & 1.874733 & -2.115670 & 0.182613 \\ 6 & \mathrm{H} & 3.328085 & -1.036008 & 0.612083 \\ 7 & \mathrm{H} & 1.900785 & 0.974441 & 0.534259 \\ 8 & \mathrm{H} & -0.058716 & 0.943777 & 2.022790 \\ 9 & \mathrm{C} & -0.233032 & -1.224967 & 2.241235 \\ 10 & \mathrm{H} & -0.357019 & -0.926055 & -0.446960 \\ 11 & \mathrm{H} & -0.371475 & 0.896819 & -0.493108 \\ 12 & \mathrm{O} & -0.256094 & -2.347748 & 1.751332 \\ 13 & \mathrm{H} & -0.380562 & -1.082019 & 3.333194\end{array}$

transition state of C-C cleavage of trans- $C_{1}$-formyl cyclopropylcarbinyl radical

\begin{tabular}{|c|c|c|c|c|}
\hline \multirow{2}{*}{$\begin{array}{l}\text { Center } \\
\text { Number }\end{array}$} & \multirow{2}{*}{$\begin{array}{l}\text { Atomic } \\
\text { Number }\end{array}$} & \multicolumn{3}{|c|}{ Coordinates (Angstroms) } \\
\hline & & $\mathrm{X}$ & Y & $\mathrm{Z}$ \\
\hline 1 & $\mathrm{C}$ & 0.000000 & 0.000000 & 0.000000 \\
\hline 2 & $\mathrm{C}$ & 0.000000 & 0.000000 & 1.489462 \\
\hline 3 & $\mathrm{C}$ & 1.434678 & 0.000000 & 0.407650 \\
\hline 4 & $\mathrm{C}$ & 2.254551 & -1.136283 & 0.428279 \\
\hline 5 & $\mathrm{H}$ & 1.856779 & -2.124768 & 0.216654 \\
\hline 6 & $\mathrm{H}$ & 3.297890 & -1.065939 & 0.715965 \\
\hline 7 & $\mathrm{H}$ & 1.887715 & 0.979842 & 0.525496 \\
\hline 8 & $\mathrm{C}$ & -0.117738 & 1.244844 & 2.232338 \\
\hline 9 & $\mathrm{H}$ & -0.097406 & -0.939216 & 2.023086 \\
\hline 10 & $\mathrm{H}$ & -0.362556 & -0.904944 & -0.484842 \\
\hline 11 & $\mathrm{H}$ & -0.360375 & 0.921558 & -0.452429 \\
\hline 12 & $\mathrm{O}$ & -0.062991 & 2.358906 & 1.719525 \\
\hline 13 & $\mathrm{H}$ & -0.248066 & 1.130828 & 3.329034 \\
\hline
\end{tabular}

transition state of C-C cleavage of $c i s-C_{1}$-phenyl cyclopropylcarbinyl radical

\begin{tabular}{ccccc} 
Center & Atomic & \multicolumn{3}{c}{ Coordinates (Angstroms) } \\
Number & Number & X & Y & Z \\
- & Nume & & \\
1 & $\mathrm{C}$ & 0.000000 & 0.000000 & 0.000000 \\
2 & $\mathrm{C}$ & 0.000000 & 0.000000 & 1.486142 \\
3 & $\mathrm{C}$ & 1.440968 & 0.000000 & 0.409193 \\
4 & $\mathrm{C}$ & 2.297207 & -1.110581 & 0.332800 \\
5 & $\mathrm{H}$ & 1.925982 & -2.102259 & 0.095464 \\
6 & $\mathrm{H}$ & 3.336856 & -1.027125 & 0.631031 \\
7 & $\mathrm{H}$ & 1.899499 & 0.976552 & 0.541706 \\
8 & $\mathrm{H}$ & -0.035455 & 0.974799 & 1.964345 \\
9 & $\mathrm{C}$ & -0.305730 & -1.133399 & 2.355583
\end{tabular}




$\begin{array}{llrrr}10 & \mathrm{H} & -0.346230 & -0.906972 & -0.494205 \\ 11 & \mathrm{H} & -0.374168 & 0.897865 & -0.492374 \\ 12 & \mathrm{C} & -0.554164 & -2.436661 & 1.880490 \\ 13 & \mathrm{C} & -0.833813 & -3.480042 & 2.760641 \\ 14 & \mathrm{C} & -0.877905 & -3.255371 & 4.138210 \\ 15 & \mathrm{C} & -0.637802 & -1.968396 & 4.627578 \\ 16 & \mathrm{C} & -0.353218 & -0.927026 & 3.749888 \\ 17 & \mathrm{H} & -0.164633 & 0.070381 & 4.141152 \\ 18 & \mathrm{H} & -0.671660 & -1.776297 & 5.697025 \\ 19 & \mathrm{H} & -1.098838 & -4.071127 & 4.821015 \\ 20 & \mathrm{H} & -1.023742 & -4.475246 & 2.366276 \\ 21 & \mathrm{H} & -0.540351 & -2.637910 & 0.813456\end{array}$

transition state of C-C cleavage of trans- $C_{1}$-phenyl cyclopropylcarbinyl radical

\begin{tabular}{|c|c|c|c|c|}
\hline \multirow{2}{*}{$\begin{array}{l}\text { Center } \\
\text { Number }\end{array}$} & \multirow{2}{*}{$\begin{array}{l}\text { Atomic } \\
\text { Number }\end{array}$} & \multicolumn{3}{|c|}{ Coordinates (Angstroms) } \\
\hline & & $X$ & Y & $\mathrm{Z}$ \\
\hline 1 & $\mathrm{C}$ & 0.000000 & 0.000000 & 0.000000 \\
\hline 2 & $\mathrm{C}$ & 0.000000 & 0.000000 & 1.487198 \\
\hline 3 & $\mathrm{C}$ & 1.437959 & 0.000000 & 0.410048 \\
\hline 4 & $\mathrm{C}$ & 2.271245 & -1.128706 & 0.386368 \\
\hline 5 & $\mathrm{H}$ & 1.880958 & -2.113501 & 0.145105 \\
\hline 6 & $\mathrm{H}$ & 3.307160 & -1.063985 & 0.701179 \\
\hline 7 & $\mathrm{H}$ & 1.897643 & 0.976222 & 0.538154 \\
\hline 8 & $\mathrm{H}$ & -0.079936 & -0.968970 & 1.968691 \\
\hline 9 & $\mathrm{C}$ & -0.207382 & 1.165178 & 2.337966 \\
\hline 10 & $\mathrm{H}$ & -0.354433 & -0.914020 & -0.476426 \\
\hline 11 & $\mathrm{H}$ & -0.371462 & 0.892863 & -0.501550 \\
\hline 12 & $\mathrm{C}$ & -0.293794 & 2.481498 & 1.835859 \\
\hline 13 & $\mathrm{C}$ & -0.482995 & 3.564236 & 2.690872 \\
\hline 14 & $\mathrm{C}$ & -0.594996 & 3.369866 & 4.069944 \\
\hline 15 & $\mathrm{C}$ & -0.512510 & 2.072791 & 4.585328 \\
\hline 16 & $\mathrm{C}$ & -0.318014 & 0.989770 & 3.734555 \\
\hline 17 & $\mathrm{H}$ & -0.253418 & -0.015176 & 4.145614 \\
\hline 18 & $\mathrm{H}$ & -0.599586 & 1.905684 & 5.655997 \\
\hline 19 & $\mathrm{H}$ & -0.745278 & 4.216713 & 4.733683 \\
\hline 20 & $\mathrm{H}$ & -0.547557 & 4.567372 & 2.276707 \\
\hline 21 & $\mathrm{H}$ & -0.214522 & 2.660933 & 0.767025 \\
\hline
\end{tabular}

transition state of C-C cleavage of oxiranylcarbinyl radical

\begin{tabular}{|c|c|c|c|}
\hline Center & Atomic & $\mathrm{Coc}$ & Angstroms) \\
\hline Number & Number & $X$ & $\mathrm{Y}$ \\
\hline
\end{tabular}




$\begin{array}{lcccc}1 & \mathrm{O} & 0.000000 & 0.000000 & 0.000000 \\ 2 & \mathrm{C} & 0.000000 & 0.000000 & 1.390468 \\ 3 & \mathrm{C} & 1.396478 & 0.000000 & 0.138895 \\ 4 & \mathrm{C} & 2.147609 & -1.109643 & -0.112396 \\ 5 & \mathrm{H} & 1.681277 & -2.063417 & -0.334708 \\ 6 & \mathrm{H} & 3.229312 & -1.059134 & -0.058193 \\ 7 & \mathrm{H} & 1.842402 & 0.986650 & 0.258530 \\ 8 & \mathrm{H} & -0.155109 & -0.953346 & 1.887162 \\ 9 & \mathrm{H} & -0.221915 & 0.939208 & 1.893708\end{array}$

transition state of C-C cleavage of $c i s-C_{l}$-formyl oxiranylcarbinyl radical

\begin{tabular}{|c|c|c|c|c|}
\hline \multirow{2}{*}{$\begin{array}{l}\text { Center } \\
\text { Number }\end{array}$} & \multirow{2}{*}{$\begin{array}{l}\text { Atomic } \\
\text { Number }\end{array}$} & \multicolumn{3}{|c|}{ Coordinates (Angstroms) } \\
\hline & & $\mathrm{X}$ & $\mathrm{Y}$ & $\mathrm{Z}$ \\
\hline 1 & $\mathrm{O}$ & 0.000000 & 0.000000 & 0.000000 \\
\hline 2 & $\mathrm{C}$ & 0.000000 & 0.000000 & 1.398041 \\
\hline 3 & $\mathrm{C}$ & 1.378497 & 0.000000 & 0.256635 \\
\hline 4 & $\mathrm{C}$ & 2.169202 & -1.099377 & -0.014314 \\
\hline 5 & $\mathrm{H}$ & 1.733710 & -2.050216 & -0.299596 \\
\hline 6 & $\mathrm{H}$ & 3.245024 & -1.029702 & 0.097313 \\
\hline 7 & $\mathrm{H}$ & 1.822295 & 0.987096 & 0.385297 \\
\hline 8 & $\mathrm{H}$ & -0.121476 & 0.961184 & 1.896307 \\
\hline 9 & $\mathrm{C}$ & -0.289480 & -1.226136 & 2.124303 \\
\hline 10 & $\mathrm{H}$ & -0.276278 & -2.151016 & 1.510480 \\
\hline 11 & $\mathrm{O}$ & -0.526480 & -1.243057 & 3.323480 \\
\hline
\end{tabular}

transition state of C-C cleavage of trans $-C_{l}$-formyl oxiranylcarbinyl radical

\begin{tabular}{|c|c|c|c|c|}
\hline \multirow{2}{*}{$\begin{array}{l}\text { Center } \\
\text { Number }\end{array}$} & \multirow{2}{*}{$\begin{array}{l}\text { Atomic } \\
\text { Number }\end{array}$} & \multicolumn{3}{|c|}{ Coordinates (Angstroms) } \\
\hline & & $\mathrm{X}$ & $\mathrm{Y}$ & $\mathrm{Z}$ \\
\hline 1 & $\mathrm{O}$ & 0.000000 & 0.000000 & 0.000000 \\
\hline 2 & $\mathrm{C}$ & 0.000000 & 0.000000 & 1.396413 \\
\hline 3 & $\mathrm{C}$ & 1.382252 & 0.000000 & 0.248208 \\
\hline 4 & $\mathrm{C}$ & 2.139675 & -1.143498 & 0.122972 \\
\hline 5 & $\mathrm{H}$ & 1.672292 & -2.108334 & -0.040437 \\
\hline 6 & $\mathrm{H}$ & 3.216542 & -1.096167 & 0.236244 \\
\hline 7 & $\mathrm{H}$ & 1.841118 & 0.986352 & 0.283785 \\
\hline 8 & $\mathrm{H}$ & -0.132275 & -0.956933 & 1.895510 \\
\hline 9 & $\mathrm{C}$ & -0.214058 & 1.241071 & 2.118902 \\
\hline 10 & $\mathrm{H}$ & -0.195306 & 2.156217 & 1.489016 \\
\hline 11 & $\mathrm{O}$ & -0.390573 & 1.285131 & 3.330225 \\
\hline
\end{tabular}

transition state of C-C cleavage of $c i s-C_{1}$-phenyl oxiranylcarbinyl radical 


\begin{tabular}{|c|c|c|c|c|}
\hline \multirow{2}{*}{$\begin{array}{l}\text { Center } \\
\text { Number }\end{array}$} & \multirow{2}{*}{$\begin{array}{l}\text { Atomic } \\
\text { Number }\end{array}$} & \multicolumn{3}{|c|}{ Coordinates (Angstroms) } \\
\hline & & $\mathrm{X}$ & $\mathrm{Y}$ & $\mathrm{Z}$ \\
\hline 1 & $\mathrm{O}$ & 0.000000 & 0.000000 & 0.000000 \\
\hline 2 & $\mathrm{C}$ & 0.000000 & 0.000000 & 1.395354 \\
\hline 3 & $\mathrm{C}$ & 1.387623 & 0.000000 & 0.263223 \\
\hline 4 & $\mathrm{C}$ & 2.180917 & -1.102956 & 0.034700 \\
\hline 5 & $\mathrm{H}$ & 1.755859 & -2.079998 & -0.161326 \\
\hline 6 & $\mathrm{H}$ & 3.256830 & -1.017102 & 0.136687 \\
\hline 7 & $\mathrm{H}$ & 1.827368 & 0.994996 & 0.335256 \\
\hline 8 & $\mathrm{H}$ & -0.061695 & 0.983348 & 1.863411 \\
\hline 9 & $\mathrm{C}$ & -0.443019 & -1.161247 & 2.137115 \\
\hline 10 & $\mathrm{C}$ & -0.773968 & -2.373609 & 1.498477 \\
\hline 11 & $\mathrm{C}$ & -1.182491 & -3.473877 & 2.246626 \\
\hline 12 & $\mathrm{C}$ & -1.269888 & -3.392118 & 3.639623 \\
\hline 13 & $\mathrm{C}$ & -0.950042 & -2.191704 & 4.282973 \\
\hline 14 & $\mathrm{C}$ & -0.542326 & -1.087983 & 3.542088 \\
\hline 15 & $\mathrm{H}$ & -0.295726 & -0.155985 & 4.045949 \\
\hline 16 & $\mathrm{H}$ & -1.021514 & -2.118027 & 5.364944 \\
\hline 17 & $\mathrm{H}$ & -1.589657 & -4.253896 & 4.218877 \\
\hline 18 & $\mathrm{H}$ & -1.440674 & -4.400654 & 1.740634 \\
\hline 19 & $\mathrm{H}$ & -0.727797 & -2.427660 & 0.415813 \\
\hline
\end{tabular}

transition state of C-C cleavage of trans- $C_{1}$-phenyl oxiranylcarbinyl radical

\begin{tabular}{|c|c|c|c|c|}
\hline \multirow{2}{*}{$\begin{array}{l}\text { Center } \\
\text { Number }\end{array}$} & \multirow{2}{*}{$\begin{array}{l}\text { Atomic } \\
\text { Number }\end{array}$} & \multicolumn{3}{|c|}{ Coordinates (Angstroms) } \\
\hline & & $X$ & $\mathrm{Y}$ & $\mathrm{Z}$ \\
\hline 1 & $\mathrm{O}$ & 0.000000 & 0.000000 & 0.000000 \\
\hline 2 & $\mathrm{C}$ & 0.000000 & 0.000000 & 1.396346 \\
\hline 3 & $\mathrm{C}$ & 1.385227 & 0.000000 & 0.267267 \\
\hline 4 & $\mathrm{C}$ & 2.156697 & -1.121986 & 0.048839 \\
\hline 5 & $\mathrm{H}$ & 1.700534 & -2.078023 & -0.183945 \\
\hline 6 & $\mathrm{H}$ & 3.232419 & -1.074119 & 0.174011 \\
\hline 7 & $\mathrm{H}$ & 1.829540 & 0.989463 & 0.360461 \\
\hline 8 & $\mathrm{H}$ & -0.092249 & -0.977128 & 1.865473 \\
\hline 9 & $\mathrm{C}$ & -0.357024 & 1.194621 & 2.129046 \\
\hline 10 & $\mathrm{C}$ & -0.574005 & 2.424711 & 1.472393 \\
\hline 11 & $\mathrm{C}$ & -0.891261 & 3.564964 & 2.203796 \\
\hline 12 & $\mathrm{C}$ & -0.998446 & 3.507313 & 3.597122 \\
\hline 13 & $\mathrm{C}$ & -0.788774 & 2.291556 & 4.257713 \\
\hline 14 & $\mathrm{C}$ & -0.471226 & 1.147266 & 3.535094 \\
\hline 15 & $\mathrm{H}$ & -0.310042 & 0.203519 & 4.051246 \\
\hline 16 & $\mathrm{H}$ & -0.876045 & 2.238500 & 5.339750 \\
\hline
\end{tabular}




$\begin{array}{lllll}17 & \mathrm{H} & -1.247386 & 4.400348 & 4.163579 \\ 18 & \mathrm{H} & -1.062302 & 4.504968 & 1.685454 \\ 19 & \mathrm{H} & -0.508442 & 2.460913 & 0.389455\end{array}$

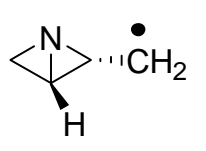

\begin{tabular}{|c|c|c|c|c|}
\hline \multirow{3}{*}{$\begin{array}{l}\text { Center } \\
\text { Number } \\
-1\end{array}$} & \multirow{3}{*}{$\begin{array}{l}\text { Atomic } \\
\text { Number } \\
\text { N }\end{array}$} & \multicolumn{3}{|c|}{ Coordinates (Angstroms) } \\
\hline & & $X$ & $\mathrm{Y}$ & $\mathrm{Z}$ \\
\hline & & 0.000000 & 0.000000 & 0.000000 \\
\hline 2 & $\mathrm{C}$ & 0.000000 & 0.000000 & 1.483908 \\
\hline 3 & $\mathrm{C}$ & 1.324466 & 0.000000 & 0.815741 \\
\hline 4 & $\mathrm{C}$ & 2.264181 & -1.105955 & 0.814118 \\
\hline 5 & $\mathrm{C}$ & -0.626385 & -1.117210 & 0.761376 \\
\hline 6 & $\mathrm{H}$ & 3.294844 & -0.931178 & 0.527216 \\
\hline 7 & $\mathrm{H}$ & 1.964970 & -2.128186 & 1.011090 \\
\hline 8 & $\mathrm{H}$ & 1.782354 & 0.983221 & 0.714339 \\
\hline 9 & $\mathrm{H}$ & -0.459055 & 0.783129 & 2.079739 \\
\hline 10 & $\mathrm{H}$ & -0.167466 & -2.104331 & 0.747244 \\
\hline 11 & $\mathrm{H}$ & -1.710923 & -1.123001 & 0.671438 \\
\hline
\end{tabular}

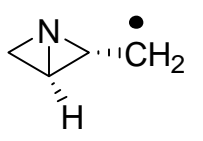

\begin{tabular}{|c|c|c|c|c|}
\hline \multirow{2}{*}{$\begin{array}{l}\text { Center } \\
\text { Number }\end{array}$} & \multirow{2}{*}{$\begin{array}{l}\text { Atomic } \\
\text { Number }\end{array}$} & \multicolumn{3}{|c|}{ Coordinates (Angstroms) } \\
\hline & & $\mathrm{X}$ & Y & $\mathrm{Z}$ \\
\hline 1 & $\mathrm{~N}$ & 0.000000 & 0.000000 & 0.000000 \\
\hline 2 & $\mathrm{C}$ & 0.000000 & 0.000000 & 1.489274 \\
\hline 3 & $\mathrm{C}$ & 1.309237 & 0.000000 & 0.780958 \\
\hline 4 & $\mathrm{C}$ & 2.113106 & -1.206262 & 0.671655 \\
\hline 5 & $\mathrm{C}$ & -0.590596 & 1.133124 & 0.765536 \\
\hline 6 & $\mathrm{H}$ & 3.194906 & -1.153372 & 0.670572 \\
\hline 7 & $\mathrm{H}$ & 1.635704 & -2.174426 & 0.567278 \\
\hline 8 & $\mathrm{H}$ & 1.857819 & 0.945906 & 0.781662 \\
\hline 9 & $\mathrm{H}$ & -0.459617 & -0.782554 & 2.082635 \\
\hline 10 & $\mathrm{H}$ & -0.069758 & 2.094119 & 0.759068 \\
\hline 11 & $\mathrm{H}$ & -1.672412 & 1.192437 & 0.671391 \\
\hline
\end{tabular}




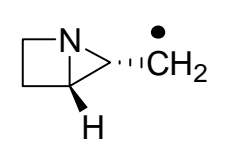

\begin{tabular}{crrrr} 
Center & Atomic & \multicolumn{3}{c}{ Coordinates (Angstroms) } \\
Number & Number & X & Y & $\mathrm{Z}$ \\
- & & & & \\
1 & $\mathrm{~N}$ & 0.000000 & 0.000000 & 0.000000 \\
2 & $\mathrm{C}$ & 0.000000 & 0.000000 & 1.504311 \\
3 & $\mathrm{C}$ & 1.313828 & 0.000000 & 0.785965 \\
4 & $\mathrm{C}$ & 2.279112 & -1.079678 & 0.794332 \\
5 & $\mathrm{H}$ & 3.322650 & -0.871083 & 0.589294 \\
6 & $\mathrm{H}$ & 2.000152 & -2.111938 & 0.969813 \\
7 & $\mathrm{H}$ & 1.747075 & 0.993197 & 0.689555 \\
8 & $\mathrm{C}$ & -0.631411 & -1.386457 & 1.599578 \\
9 & $\mathrm{H}$ & -0.314367 & 0.871377 & 2.078085 \\
10 & $\mathrm{C}$ & -0.548906 & -1.405564 & 0.051029 \\
11 & $\mathrm{H}$ & 0.095945 & -2.155594 & -0.417233 \\
12 & $\mathrm{H}$ & -1.524977 & -1.419849 & -0.440476 \\
13 & $\mathrm{H}$ & -1.659491 & -1.361978 & 1.969879 \\
14 & $\mathrm{H}$ & -0.074864 & -2.145873 & 2.159805
\end{tabular}

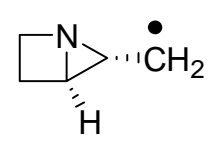

\begin{tabular}{|c|c|c|c|c|}
\hline \multirow{2}{*}{$\begin{array}{l}\text { Center } \\
\text { Number }\end{array}$} & \multirow{2}{*}{$\begin{array}{l}\text { Atomic } \\
\text { Number }\end{array}$} & \multicolumn{3}{|c|}{ Coordinates (Angstroms) } \\
\hline & & $\mathrm{X}$ & $\mathrm{Y}$ & $\mathrm{Z}$ \\
\hline 1 & $\mathrm{~N}$ & 0.000000 & 0.000000 & 0.000000 \\
\hline 2 & $\mathrm{C}$ & 0.000000 & 0.000000 & 1.506942 \\
\hline 3 & $\mathrm{C}$ & 1.299013 & 0.000000 & 0.749167 \\
\hline 4 & $\mathrm{C}$ & 2.097761 & -1.211806 & 0.636313 \\
\hline 5 & $\mathrm{H}$ & 3.161661 & -1.192832 & 0.841520 \\
\hline 6 & $\mathrm{H}$ & 1.623509 & -2.159181 & 0.406343 \\
\hline 7 & $\mathrm{H}$ & 1.859611 & 0.937095 & 0.762771 \\
\hline 8 & $\mathrm{H}$ & -0.314705 & -0.870970 & 2.078414 \\
\hline 9 & $\mathrm{C}$ & -0.589816 & 1.402985 & 1.605190 \\
\hline 10 & $\mathrm{C}$ & -0.494223 & 1.424400 & 0.055424 \\
\hline 11 & $\mathrm{H}$ & 0.197696 & 2.145079 & -0.395714 \\
\hline 12 & $\mathrm{H}$ & -1.462073 & 1.498527 & -0.447311 \\
\hline 13 & $\mathrm{H}$ & -0.011337 & 2.142590 & 2.170682 \\
\hline 14 & $\mathrm{H}$ & -1.622255 & 1.413279 & 1.965173 \\
\hline
\end{tabular}




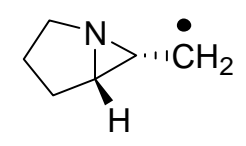

\begin{tabular}{crrrr} 
Center & Atomic & \multicolumn{3}{c}{ Coordinates (Angstroms) } \\
Number & Number & X & Y & Z \\
- & Nume & & \\
1 & $\mathrm{~N}$ & 0.000000 & 0.000000 & 0.000000 \\
2 & $\mathrm{C}$ & 0.000000 & 0.000000 & 1.460234 \\
3 & $\mathrm{C}$ & 1.332157 & 0.000000 & 0.743871 \\
4 & $\mathrm{C}$ & 2.281988 & -1.091125 & 0.751694 \\
5 & $\mathrm{H}$ & 2.003491 & -2.122384 & 0.928037 \\
6 & $\mathrm{H}$ & 3.328879 & -0.884092 & 0.560664 \\
7 & $\mathrm{H}$ & 1.790799 & 0.981567 & 0.639809 \\
8 & $\mathrm{H}$ & -0.303507 & 0.927814 & 1.946224 \\
9 & $\mathrm{C}$ & -0.567081 & -1.302083 & 2.012781 \\
10 & $\mathrm{C}$ & -0.493683 & -1.319081 & -0.445863 \\
11 & $\mathrm{H}$ & 0.180925 & -1.738044 & -1.199722 \\
12 & $\mathrm{H}$ & -1.458077 & -1.133121 & -0.932297 \\
13 & $\mathrm{H}$ & 0.109761 & -3.016768 & 0.815910 \\
14 & $\mathrm{C}$ & -0.667106 & -2.247122 & 0.787292 \\
15 & $\mathrm{H}$ & -1.626904 & -2.773258 & 0.760450 \\
16 & $\mathrm{H}$ & 0.052318 & -1.721994 & 2.813353 \\
17 & $\mathrm{H}$ & -1.556211 & -1.105002 & 2.441567
\end{tabular}

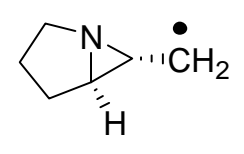

\begin{tabular}{|c|c|c|}
\hline Center & Atomic & Coordinates (Angstroms) \\
\hline Number & Number & $X$ \\
\hline
\end{tabular}

$\begin{array}{rrrrr}1 & \mathrm{~N} & 0.000000 & 0.000000 & 0.000000 \\ 2 & \mathrm{C} & 0.000000 & 0.000000 & 1.465658 \\ 3 & \mathrm{C} & 1.314195 & 0.000000 & 0.704795 \\ 4 & \mathrm{C} & 2.143142 & -1.187444 & 0.562449 \\ 5 & \mathrm{H} & 1.691660 & -2.136947 & 0.297453 \\ 6 & \mathrm{H} & 3.199641 & -1.158197 & 0.804935 \\ 7 & \mathrm{H} & 1.857059 & 0.945770 & 0.743310 \\ 8 & \mathrm{H} & -0.316243 & -0.920837 & 1.952450 \\ 9 & \mathrm{C} & -0.508272 & 1.330963 & 2.005802 \\ 10 & \mathrm{C} & -0.446374 & 1.344279 & -0.424013 \\ 11 & \mathrm{C} & -0.373917 & 2.290120 & 0.801006 \\ 12 & \mathrm{H} & 0.583879 & 2.819951 & 0.833261 \\ 13 & \mathrm{H} & -1.482383 & 1.237416 & -0.767782\end{array}$




$\begin{array}{llrrr}14 & \mathrm{H} & 0.151931 & 1.690105 & -1.273816 \\ 15 & \mathrm{H} & -1.157828 & 3.053399 & 0.780285 \\ 16 & \mathrm{H} & 0.043979 & 1.676342 & 2.887193 \\ 17 & \mathrm{H} & -1.558880 & 1.219084 & 2.302635\end{array}$

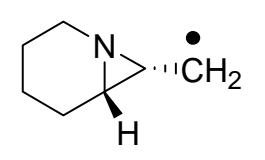

\begin{tabular}{crrrr} 
Center & Atomic & \multicolumn{3}{c}{ Coordinates (Angstroms) } \\
Number & Number & X & Y & Z \\
----------1 & \\
1 & N & 0.000000 & 0.000000 & 0.000000 \\
2 & $\mathrm{C}$ & 0.000000 & 0.000000 & 1.456548 \\
3 & $\mathrm{C}$ & 1.328541 & 0.000000 & 0.719389 \\
4 & $\mathrm{C}$ & 2.254730 & -1.110981 & 0.728507 \\
5 & $\mathrm{H}$ & 1.939332 & -2.144681 & 0.790970 \\
6 & $\mathrm{H}$ & 3.318850 & -0.918475 & 0.652928 \\
7 & $\mathrm{H}$ & 1.808543 & 0.974081 & 0.632485 \\
8 & $\mathrm{H}$ & -0.276710 & 0.970370 & 1.875585 \\
9 & $\mathrm{C}$ & -0.527295 & -1.172846 & 2.279059 \\
10 & $\mathrm{C}$ & -0.449245 & -1.214141 & -0.710412 \\
11 & $\mathrm{H}$ & 0.412449 & -1.830916 & -0.997892 \\
12 & $\mathrm{H}$ & -0.920232 & -0.876751 & -1.641789 \\
13 & $\mathrm{C}$ & -1.438210 & -2.059980 & 0.098642 \\
14 & $\mathrm{H}$ & -1.703685 & -2.956035 & -0.475947 \\
15 & $\mathrm{H}$ & -2.366911 & -1.491611 & 0.249696 \\
16 & $\mathrm{C}$ & -0.842176 & -2.438299 & 1.457995 \\
17 & $\mathrm{H}$ & 0.062919 & -3.038907 & 1.301697 \\
18 & $\mathrm{H}$ & -1.535399 & -3.072370 & 2.023713 \\
19 & $\mathrm{H}$ & 0.166966 & -1.399120 & 3.098630 \\
20 & $\mathrm{H}$ & -1.458967 & -0.835032 & 2.751730
\end{tabular}<smiles>[CH2][C@@H]1[C@H]2CCCCN12</smiles>

\begin{tabular}{ccccc}
$\begin{array}{l}\text { Center } \\
\text { Number }\end{array}$ & $\begin{array}{c}\text { Atomic } \\
\text { Number }\end{array}$ & \multicolumn{4}{c}{ Coordinates (Angstroms) } \\
\hdashline & X & Y & Z \\
\hline 1 & N & 0.000000 & 0.000000 & 0.000000 \\
2 & $\mathrm{C}$ & 0.000000 & 0.000000 & 1.454081 \\
3 & $\mathrm{C}$ & 1.317237 & 0.000000 & 0.682353 \\
4 & $\mathrm{C}$ & 2.151046 & -1.184479 & 0.536663 \\
5 & $\mathrm{H}$ & 1.704056 & -2.132839 & 0.259867
\end{tabular}




$\begin{array}{rrrrr}6 & \mathrm{H} & 3.201826 & -1.158474 & 0.804412 \\ 7 & \mathrm{H} & 1.858810 & 0.944306 & 0.726623 \\ 8 & \mathrm{H} & -0.303623 & -0.950346 & 1.896333 \\ 9 & \mathrm{C} & -0.504060 & 1.233254 & 2.183850 \\ 10 & \mathrm{C} & -0.471981 & 1.247678 & -0.618839 \\ 11 & \mathrm{C} & -0.002010 & 2.571848 & 0.021043 \\ 12 & \mathrm{C} & 0.000573 & 2.556712 & 1.578219 \\ 13 & \mathrm{H} & -0.607411 & 3.379453 & 1.970718 \\ 14 & \mathrm{H} & 1.019717 & 2.740570 & 1.938404 \\ 15 & \mathrm{H} & 1.005213 & 2.807355 & -0.342004 \\ 16 & \mathrm{H} & -0.646963 & 3.374363 & -0.356374 \\ 17 & \mathrm{H} & -0.182608 & 1.226411 & -1.675934 \\ 18 & \mathrm{H} & -1.568464 & 1.193582 & -0.592961 \\ 19 & \mathrm{H} & -1.603761 & 1.206913 & 2.152350 \\ 20 & \mathrm{H} & -0.231407 & 1.178630 & 3.245412\end{array}$

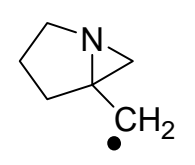

\begin{tabular}{lllcl} 
Center & Atomic & \multicolumn{2}{c}{ Coordinates (Angstroms) } \\
Number & Number & $\mathrm{X}$ & $\mathrm{Y}$ & $\mathrm{Z}$
\end{tabular}

$\begin{array}{rrrrr}1 & \mathrm{~N} & 0.000000 & 0.000000 & 0.000000 \\ 2 & \mathrm{C} & 0.000000 & 0.000000 & 1.458668 \\ 3 & \mathrm{C} & 1.327636 & 0.000000 & 0.747748 \\ 4 & \mathrm{C} & 2.158531 & -1.192847 & 0.696963 \\ 5 & \mathrm{H} & 1.711786 & -2.178662 & 0.761992 \\ 6 & \mathrm{H} & 3.231126 & -1.119300 & 0.552942 \\ 7 & \mathrm{C} & 1.983439 & 1.372065 & 0.581245 \\ 8 & \mathrm{H} & -0.350989 & -0.926567 & 1.909249 \\ 9 & \mathrm{H} & -0.298183 & 0.897994 & 2.001619 \\ 10 & \mathrm{C} & -0.142135 & 1.345326 & -0.590552 \\ 11 & \mathrm{C} & 0.847259 & 2.304108 & 0.113917 \\ 12 & \mathrm{H} & 0.373742 & 2.798075 & 0.969151 \\ 13 & \mathrm{H} & 1.199490 & 3.096786 & -0.553435 \\ 14 & \mathrm{H} & 0.101010 & 1.251807 & -1.655319 \\ 15 & \mathrm{H} & -1.185078 & 1.675980 & -0.514891 \\ 16 & \mathrm{H} & 2.766299 & 1.297574 & -0.183838 \\ 17 & \mathrm{H} & 2.461469 & 1.721968 & 1.503396\end{array}$

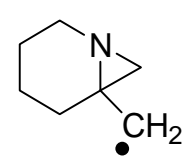




\begin{tabular}{|c|c|c|c|c|}
\hline \multirow{2}{*}{$\begin{array}{l}\text { Center } \\
\text { Number }\end{array}$} & \multirow{2}{*}{$\begin{array}{l}\text { Atomic } \\
\text { Number }\end{array}$} & \multicolumn{3}{|c|}{ Coordinates (Angstroms) } \\
\hline & & $X$ & Y & $\mathrm{Z}$ \\
\hline 1 & $\mathrm{~N}$ & 0.000000 & 0.000000 & 0.000000 \\
\hline 2 & $\mathrm{C}$ & 0.000000 & 0.000000 & 1.455153 \\
\hline 3 & $\mathrm{C}$ & 1.315214 & 0.000000 & 0.694226 \\
\hline 4 & $\mathrm{C}$ & 2.085551 & -1.240154 & 0.579743 \\
\hline 5 & $\mathrm{H}$ & 1.579926 & -2.172415 & 0.354257 \\
\hline 6 & $\mathrm{H}$ & 3.147813 & -1.258926 & 0.800925 \\
\hline 7 & $\mathrm{C}$ & 2.128286 & 1.293003 & 0.625042 \\
\hline 8 & $\mathrm{H}$ & -0.343878 & -0.923181 & 1.917616 \\
\hline 9 & $\mathrm{H}$ & -0.278463 & 0.901630 & 1.998588 \\
\hline 10 & $\mathrm{C}$ & -0.325394 & 1.248315 & -0.705041 \\
\hline 11 & $\mathrm{C}$ & -0.047471 & 2.568793 & 0.044042 \\
\hline 12 & $\mathrm{C}$ & 1.303087 & 2.576853 & 0.817279 \\
\hline 13 & $\mathrm{H}$ & 1.108289 & 2.716457 & 1.887221 \\
\hline 14 & $\mathrm{H}$ & 1.913792 & 3.433904 & 0.511908 \\
\hline 15 & $\mathrm{H}$ & 2.624092 & 1.314465 & -0.355722 \\
\hline 16 & $\mathrm{H}$ & 2.933930 & 1.257950 & 1.369526 \\
\hline 17 & $\mathrm{H}$ & 0.246361 & 1.221810 & -1.641280 \\
\hline 18 & $\mathrm{H}$ & -0.079368 & 3.383981 & -0.688516 \\
\hline 19 & $\mathrm{H}$ & -1.384931 & 1.201830 & -0.985777 \\
\hline 20 & $\mathrm{H}$ & -0.869657 & 2.766010 & 0.741953 \\
\hline
\end{tabular}

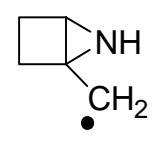

\begin{tabular}{|c|c|c|c|c|}
\hline \multirow{2}{*}{$\begin{array}{l}\text { Center } \\
\text { Number }\end{array}$} & \multirow{2}{*}{$\begin{array}{l}\text { Atomic } \\
\text { Number }\end{array}$} & \multicolumn{3}{|c|}{ Coordinates (Angstroms) } \\
\hline & & $X$ & $\mathrm{Y}$ & $\mathrm{Z}$ \\
\hline 1 & $\mathrm{~N}$ & 0.000000 & 0.000000 & 0.000000 \\
\hline 2 & $\mathrm{C}$ & 0.000000 & 0.000000 & 1.445536 \\
\hline 3 & $\mathrm{C}$ & 1.332098 & 0.000000 & 0.613993 \\
\hline 4 & $\mathrm{C}$ & 2.268773 & 1.042512 & 0.306536 \\
\hline 5 & $\mathrm{H}$ & 3.280482 & 1.005017 & 0.696126 \\
\hline 6 & $\mathrm{H}$ & 1.942306 & 1.929247 & -0.223906 \\
\hline 7 & $\mathrm{C}$ & 1.662198 & -1.388555 & 1.202399 \\
\hline 8 & $\mathrm{C}$ & 0.341130 & -1.369623 & 2.032842 \\
\hline 9 & $\mathrm{H}$ & -0.480907 & 0.829141 & 1.957269 \\
\hline 10 & $\mathrm{H}$ & -0.247371 & -0.910477 & -0.397651 \\
\hline 11 & $\mathrm{H}$ & 0.526173 & -1.342650 & 3.110671 \\
\hline 12 & $\mathrm{H}$ & -0.387785 & -2.164396 & 1.825048 \\
\hline 13 & $\mathrm{H}$ & 2.552251 & -1.378058 & 1.838525 \\
\hline 14 & $\mathrm{H}$ & 1.775003 & -2.188406 & 0.459294 \\
\hline
\end{tabular}




\begin{tabular}{rrrrr}
\hline & \multicolumn{4}{c}{} \\
\hline \\
\hline
\end{tabular}

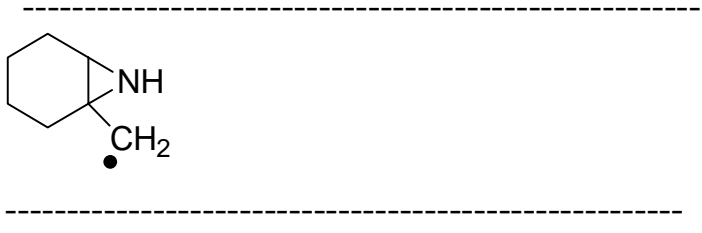

\begin{tabular}{|c|c|c|c|c|}
\hline \multirow{2}{*}{$\begin{array}{l}\text { Center } \\
\text { Number }\end{array}$} & \multirow{2}{*}{$\begin{array}{l}\text { Atomic } \\
\text { Number }\end{array}$} & \multicolumn{3}{|c|}{ Coordinates (Angstroms) } \\
\hline & & $X$ & Y & $\mathrm{Z}$ \\
\hline 1 & $\mathrm{~N}$ & 0.000000 & 0.000000 & 0.000000 \\
\hline 2 & $\mathrm{C}$ & 0.000000 & 0.000000 & 1.464530 \\
\hline 3 & $\mathrm{C}$ & 1.323352 & 0.000000 & 0.752038 \\
\hline 4 & $\mathrm{C}$ & 2.121198 & 1.213351 & 0.736407 \\
\hline 5 & $\mathrm{H}$ & 1.657062 & 2.184056 & 0.886930 \\
\hline 6 & $\mathrm{H}$ & 3.182759 & 1.184138 & 0.516138 \\
\hline 7 & $\mathrm{C}$ & 2.060073 & -1.334885 & 0.730660 \\
\hline 8 & $\mathrm{C}$ & -0.419960 & -1.282368 & 2.159150 \\
\hline 9 & $\mathrm{H}$ & -0.282869 & 0.923147 & 1.973200 \\
\hline 10 & $\mathrm{H}$ & -0.174477 & 0.943141 & -0.352269 \\
\hline 11 & $\mathrm{H}$ & -1.480211 & -1.227777 & 2.440818 \\
\hline 12 & $\mathrm{H}$ & 0.143434 & -1.347016 & 3.101133 \\
\hline
\end{tabular}




\begin{tabular}{|c|c|c|c|c|}
\hline 13 & $\mathrm{C}$ & -0.182888 & -2.540945 & 1.299515 \\
\hline 14 & $\mathrm{H}$ & -0.212541 & -3.416472 & 1.958820 \\
\hline 15 & $\mathrm{H}$ & -1.025710 & -2.645620 & 0.609421 \\
\hline 16 & $\mathrm{H}$ & 1.699553 & -3.455610 & 0.646273 \\
\hline 17 & $\mathrm{H}$ & 2.570334 & -1.447125 & 1.698081 \\
\hline 18 & $\mathrm{H}$ & 2.849598 & -1.312817 & -0.032300 \\
\hline 19 & $\mathrm{H}$ & 0.873086 & -2.527506 & -0.595052 \\
\hline 20 & $\mathrm{C}$ & 1.133401 & -2.533726 & 0.467160 \\
\hline itio & te of C-N & age of & & \\
\hline Center & Atomic & Coor & linates (Angs & coms) \\
\hline Number & Number & $X$ & $\mathrm{Y}$ & $\mathrm{Z}$ \\
\hline 1 & $\mathrm{~N}$ & 0.000000 & 0.000000 & 0.000000 \\
\hline 2 & $\mathrm{C}$ & 0.000000 & 0.000000 & 1.471458 \\
\hline 3 & $\mathrm{C}$ & 1.402772 & 0.000000 & 1.030288 \\
\hline 4 & $\mathrm{C}$ & 2.290209 & -1.095727 & 1.029805 \\
\hline 5 & $\mathrm{C}$ & -0.612162 & -1.127039 & 0.749828 \\
\hline 6 & $\mathrm{H}$ & 3.317215 & -0.959655 & 0.709259 \\
\hline 7 & $\mathrm{H}$ & 1.967274 & -2.106647 & 1.250041 \\
\hline 8 & $\mathrm{H}$ & 1.836707 & 0.988166 & 0.895854 \\
\hline 9 & $\mathrm{H}$ & -0.493153 & 0.758490 & 2.074394 \\
\hline 10 & $\mathrm{H}$ & -0.149865 & -2.111660 & 0.727728 \\
\hline 11 & $\mathrm{H}$ & -1.698130 & -1.135312 & 0.668171 \\
\hline
\end{tabular}

$\begin{array}{lcccc}\text { Center } & \text { Atomic } & \text { Coordinates (Angstroms) } & \\ \text { Number } & \text { Number } & \mathrm{X} & \mathrm{Y} & \mathrm{Z}\end{array}$

$\begin{array}{rrrrr}1 & \mathrm{~N} & 0.000000 & 0.000000 & 0.000000 \\ 2 & \mathrm{C} & 0.000000 & 0.000000 & 1.469536 \\ 3 & \mathrm{C} & 1.417953 & 0.000000 & 1.088031 \\ 4 & \mathrm{C} & 2.218350 & -1.152221 & 1.067220 \\ 5 & \mathrm{C} & -0.552430 & 1.155597 & 0.746192 \\ 6 & \mathrm{H} & 3.276921 & -1.088045 & 0.842575 \\ 7 & \mathrm{H} & 1.789319 & -2.138290 & 1.213933 \\ 8 & \mathrm{H} & 1.904338 & 0.968493 & 0.992191 \\ 9 & \mathrm{H} & -0.512899 & -0.734813 & 2.084289 \\ 10 & \mathrm{H} & -0.020135 & 2.109048 & 0.743711\end{array}$




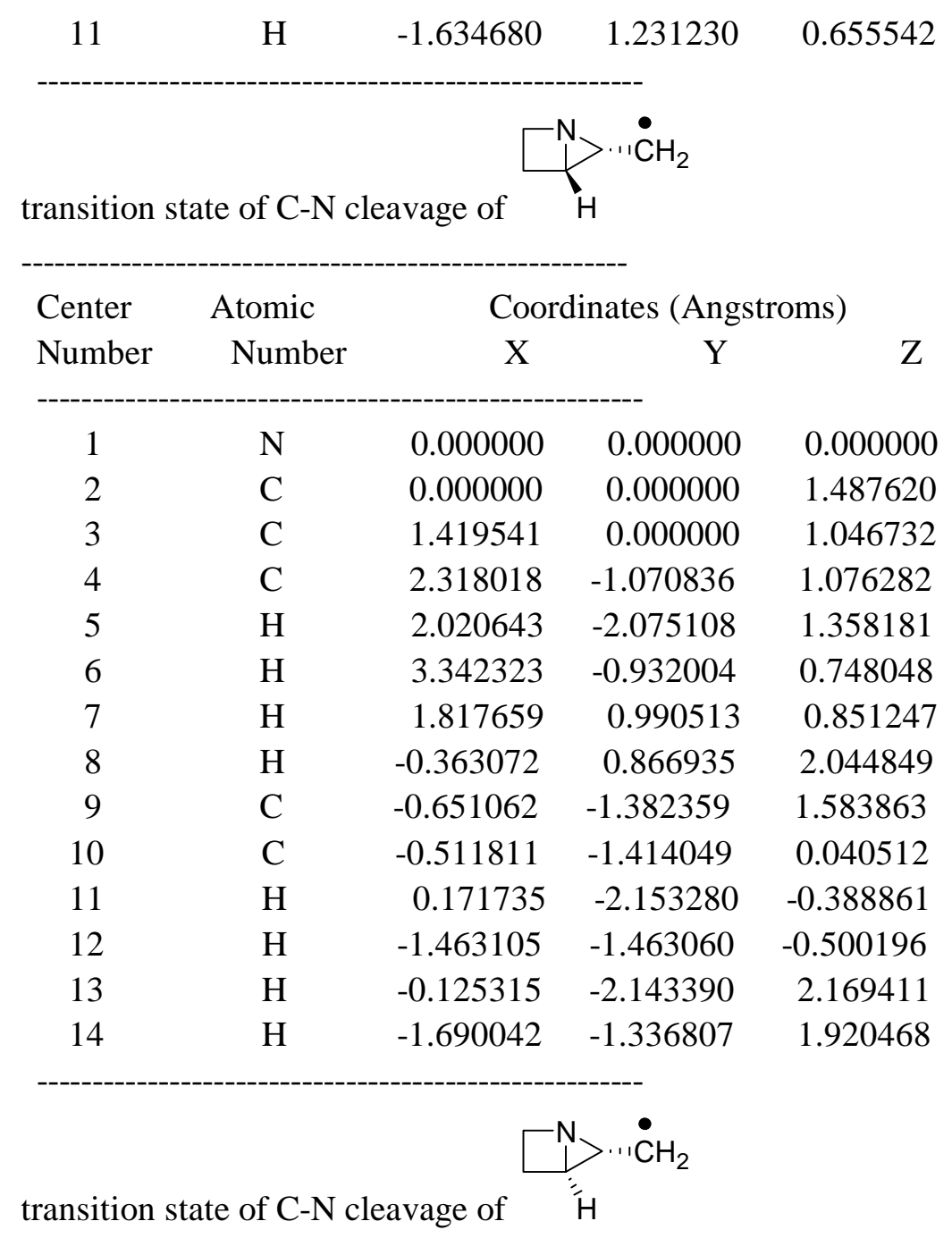

\begin{tabular}{|c|c|c|c|c|}
\hline \multirow{3}{*}{$\begin{array}{l}\text { Center } \\
\text { Number } \\
-1 \\
1\end{array}$} & \multirow{3}{*}{$\begin{array}{l}\text { Atomic } \\
\text { Number } \\
\mathrm{N}\end{array}$} & \multicolumn{3}{|c|}{ Coordinates (Angstroms) } \\
\hline & & $\mathrm{X}$ & $\mathrm{Y}$ & $\mathrm{Z}$ \\
\hline & & 0.000000 & 0.000000 & 0.000000 \\
\hline 2 & $\mathrm{C}$ & 0.000000 & 0.000000 & 1.485535 \\
\hline 3 & $\mathrm{C}$ & 1.427509 & 0.000000 & 1.081268 \\
\hline 4 & $\mathrm{C}$ & 2.217149 & -1.151203 & 1.029547 \\
\hline 5 & $\mathrm{H}$ & 1.790745 & -2.135174 & 1.197218 \\
\hline 6 & $\mathrm{H}$ & 3.259469 & -1.097469 & 0.734788 \\
\hline 7 & $\mathrm{H}$ & 1.913486 & 0.966632 & 0.978583 \\
\hline 8 & $\mathrm{H}$ & -0.374727 & -0.863822 & 2.039750 \\
\hline 9 & $\mathrm{C}$ & -0.605613 & 1.401975 & 1.583867 \\
\hline 10 & $\mathrm{C}$ & -0.409693 & 1.448632 & 0.045222 \\
\hline 11 & $\mathrm{H}$ & -1.327748 & 1.590168 & -0.536600 \\
\hline 12 & $\mathrm{H}$ & 0.346490 & 2.145036 & -0.336991 \\
\hline 13 & $\mathrm{H}$ & -0.070368 & 2.132591 & 2.199663 \\
\hline 14 & $\mathrm{H}$ & -1.658875 & 1.391881 & 1.876732 \\
\hline
\end{tabular}




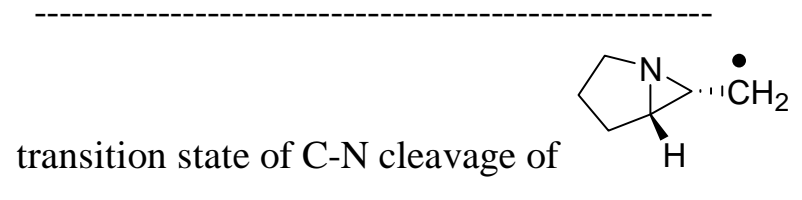

\begin{tabular}{|c|c|c|c|c|}
\hline \multirow{2}{*}{$\begin{array}{l}\text { Center } \\
\text { Number }\end{array}$} & \multirow{2}{*}{$\begin{array}{l}\text { Atomic } \\
\text { Number }\end{array}$} & \multicolumn{3}{|c|}{ Coordinates (Angstroms) } \\
\hline & & $\mathrm{X}$ & $\mathrm{Y}$ & $\mathrm{Z}$ \\
\hline 1 & $\mathrm{~N}$ & 0.000000 & 0.000000 & 0.000000 \\
\hline 2 & $\mathrm{C}$ & 0.000000 & 0.000000 & 1.453495 \\
\hline 3 & $\mathrm{C}$ & 1.430162 & 0.000000 & 1.009062 \\
\hline 4 & $\mathrm{C}$ & 2.325638 & -1.074706 & 1.058577 \\
\hline 5 & $\mathrm{H}$ & 3.352057 & -0.938724 & 0.734685 \\
\hline 6 & $\mathrm{H}$ & 2.039347 & -2.068173 & 1.383365 \\
\hline 7 & $\mathrm{H}$ & 1.846065 & 0.981975 & 0.809138 \\
\hline 8 & $\mathrm{H}$ & -0.340377 & 0.929621 & 1.916812 \\
\hline 9 & $\mathrm{C}$ & -0.627260 & -1.283231 & 1.998808 \\
\hline 10 & $\mathrm{C}$ & -0.430747 & -1.337367 & -0.439527 \\
\hline 11 & $\mathrm{H}$ & 0.277595 & -1.750262 & -1.165979 \\
\hline 12 & $\mathrm{H}$ & -1.378667 & -1.177513 & -0.972173 \\
\hline 13 & $\mathrm{H}$ & 0.177760 & -2.985794 & 0.869355 \\
\hline 14 & $\mathrm{C}$ & -0.632288 & -2.254738 & 0.793676 \\
\hline 15 & $\mathrm{H}$ & -1.566476 & -2.822391 & 0.732025 \\
\hline 16 & $\mathrm{H}$ & -0.083350 & -1.680462 & 2.862800 \\
\hline 17 & $\mathrm{H}$ & -1.648164 & -1.064260 & 2.331608 \\
\hline
\end{tabular}

\begin{tabular}{|c|c|c|c|c|}
\hline \multirow{2}{*}{$\begin{array}{l}\text { Center } \\
\text { Number }\end{array}$} & \multirow{2}{*}{$\begin{array}{l}\text { Atomic } \\
\text { Number }\end{array}$} & \multicolumn{3}{|c|}{ Coordinates (Angstroms) } \\
\hline & & $\mathrm{X}$ & Y & $\mathrm{Z}$ \\
\hline 1 & $\mathrm{~N}$ & 0.000000 & 0.000000 & 0.000000 \\
\hline 2 & $\mathrm{C}$ & 0.000000 & 0.000000 & 1.454919 \\
\hline 3 & $\mathrm{C}$ & 1.436659 & 0.000000 & 1.056255 \\
\hline 4 & $\mathrm{C}$ & 2.244992 & -1.138419 & 0.997568 \\
\hline 5 & $\mathrm{H}$ & 1.830055 & -2.130416 & 1.145777 \\
\hline 6 & $\mathrm{H}$ & 3.290242 & -1.066837 & 0.715955 \\
\hline 7 & $\mathrm{H}$ & 1.915204 & 0.971023 & 0.967654 \\
\hline 8 & $\mathrm{H}$ & -0.358193 & -0.924547 & 1.913872 \\
\hline 9 & $\mathrm{C}$ & -0.591605 & 1.303489 & 1.989484 \\
\hline 10 & $\mathrm{C}$ & -0.317406 & 1.380933 & -0.417700 \\
\hline 11 & $\mathrm{H}$ & -1.309451 & 1.326740 & -0.890941 \\
\hline 12 & $\mathrm{H}$ & 0.380929 & 1.725770 & -1.189017 \\
\hline
\end{tabular}




\begin{tabular}{|c|c|c|c|c|}
\hline 13 & $\mathrm{H}$ & 0.595879 & 2.824080 & 0.958247 \\
\hline 14 & $\mathrm{C}$ & -0.355440 & 2.296104 & 0.830824 \\
\hline 15 & $\mathrm{H}$ & -1.135875 & 3.060502 & 0.764959 \\
\hline 16 & $\mathrm{H}$ & -0.136826 & 1.624168 & 2.933075 \\
\hline 17 & $\mathrm{H}$ & -1.664246 & 1.161882 & 2.169303 \\
\hline Center & Atomic & Coor & linates (Angst & roms) \\
\hline Number & Number & $\mathrm{X}$ & Y & $\mathrm{Z}$ \\
\hline 1 & $\mathrm{~N}$ & 0.000000 & 0.000000 & 0.000000 \\
\hline 2 & $\mathrm{C}$ & 0.000000 & 0.000000 & 1.450226 \\
\hline 3 & $\mathrm{C}$ & 1.443091 & 0.000000 & 1.023620 \\
\hline 4 & $\mathrm{C}$ & 2.307083 & -1.092079 & 1.042444 \\
\hline 5 & $\mathrm{H}$ & 1.990192 & -2.087346 & 1.333379 \\
\hline 6 & $\mathrm{H}$ & 3.337087 & -0.976861 & 0.722153 \\
\hline 7 & $\mathrm{H}$ & 1.869593 & 0.978608 & 0.831112 \\
\hline 8 & $\mathrm{H}$ & -0.319797 & 0.974214 & 1.832389 \\
\hline 9 & $\mathrm{C}$ & -0.596548 & -1.165405 & 2.254400 \\
\hline 10 & $\mathrm{C}$ & -0.363362 & -1.230950 & -0.707924 \\
\hline 11 & $\mathrm{H}$ & -1.233734 & -0.936919 & -1.315494 \\
\hline 12 & $\mathrm{H}$ & 0.434441 & -1.471987 & -1.421341 \\
\hline 13 & $\mathrm{C}$ & -0.750221 & -2.468824 & 0.122317 \\
\hline 14 & $\mathrm{C}$ & -1.504047 & -2.050233 & 1.387275 \\
\hline 15 & $\mathrm{H}$ & 0.205935 & -1.782327 & 2.677651 \\
\hline 16 & $\mathrm{H}$ & -1.155127 & -0.761364 & 3.106020 \\
\hline 17 & $\mathrm{H}$ & -2.411961 & -1.496210 & 1.108473 \\
\hline 18 & $\mathrm{H}$ & -1.830649 & -2.927439 & 1.958197 \\
\hline 19 & $\mathrm{H}$ & -1.360531 & -3.136199 & -0.499099 \\
\hline 20 & $\mathrm{H}$ & 0.145292 & -3.037404 & 0.405455 \\
\hline
\end{tabular}

\begin{tabular}{ccccc}
$\begin{array}{c}\text { Center } \\
\text { Number }\end{array}$ & $\begin{array}{r}\text { Atomic } \\
\text { Number }\end{array}$ & \multicolumn{3}{c}{ Coordinates (Angstroms) } \\
$-\mathrm{X}$ & $\mathrm{Y}$ & $\mathrm{Z}$ \\
1 & $\mathrm{~N}$ & 0.000000 & 0.000000 & 0.000000 \\
2 & $\mathrm{C}$ & 0.000000 & 0.000000 & 1.452772 \\
3 & $\mathrm{C}$ & 1.442075 & 0.000000 & 1.060563 \\
4 & $\mathrm{C}$ & 2.262280 & -1.124965 & 0.996814 \\
& & & &
\end{tabular}




\begin{tabular}{|c|c|c|c|c|}
\hline 5 & $\mathrm{H}$ & 1.858445 & -2.122343 & 1.140212 \\
\hline 6 & $\mathrm{H}$ & 3.307368 & -1.040097 & 0.717817 \\
\hline 7 & $\mathrm{H}$ & 1.903001 & 0.980978 & 0.976479 \\
\hline 8 & $\mathrm{H}$ & -0.333584 & -0.970668 & 1.830899 \\
\hline 9 & $\mathrm{C}$ & -0.556933 & 1.179762 & 2.274717 \\
\hline 10 & $\mathrm{C}$ & -0.218957 & 1.279872 & -0.682954 \\
\hline 11 & $\mathrm{H}$ & 0.548267 & 1.393408 & -1.459953 \\
\hline 12 & $\mathrm{H}$ & -1.172430 & 1.147987 & -1.221521 \\
\hline 13 & $\mathrm{C}$ & -0.332329 & 2.547990 & 0.178653 \\
\hline 14 & $\mathrm{H}$ & -0.753632 & 3.356623 & -0.431574 \\
\hline 15 & $\mathrm{H}$ & 0.659082 & 2.891572 & 0.507106 \\
\hline 16 & $\mathrm{C}$ & -1.211072 & 2.259864 & 1.398902 \\
\hline 17 & $\mathrm{H}$ & -2.197235 & 1.916627 & 1.055062 \\
\hline 18 & $\mathrm{H}$ & -1.381640 & 3.166968 & 1.990499 \\
\hline 19 & $\mathrm{H}$ & -1.278878 & 0.801170 & 3.007107 \\
\hline 20 & $\mathrm{H}$ & 0.260484 & 1.630361 & 2.852976 \\
\hline Center & Atomic & \multicolumn{3}{|c|}{ Coordinates (Angstroms) } \\
\hline Number & Number & $X$ & Y & $\mathrm{Z}$ \\
\hline 1 & $\mathrm{~N}$ & 0.000000 & 0.000000 & 0.000000 \\
\hline 2 & $\mathrm{C}$ & 0.000000 & 0.000000 & 1.448012 \\
\hline 3 & $\mathrm{C}$ & 1.440693 & 0.000000 & 1.054450 \\
\hline 4 & $\mathrm{C}$ & 2.219687 & -1.164931 & 1.059912 \\
\hline 5 & $\mathrm{H}$ & 1.788955 & -2.129151 & 1.308688 \\
\hline 6 & $\mathrm{H}$ & 3.254702 & -1.144536 & 0.732549 \\
\hline 7 & $\mathrm{C}$ & 2.061691 & 1.363902 & 0.800042 \\
\hline 8 & $\mathrm{H}$ & -0.377776 & -0.932141 & 1.871625 \\
\hline 9 & $\mathrm{H}$ & -0.383655 & 0.874694 & 1.984321 \\
\hline 10 & $\mathrm{C}$ & 0.025693 & 1.350374 & -0.572219 \\
\hline 11 & $\mathrm{H}$ & -1.000851 & 1.748921 & -0.600936 \\
\hline 12 & $\mathrm{H}$ & 0.365327 & 1.255169 & -1.610232 \\
\hline 13 & $\mathrm{H}$ & 1.374064 & 3.082704 & -0.394095 \\
\hline 14 & $\mathrm{C}$ & 0.963795 & 2.281187 & 0.228828 \\
\hline 15 & $\mathrm{H}$ & 0.417850 & 2.767674 & 1.044388 \\
\hline 16 & $\mathrm{H}$ & 2.890681 & 1.253234 & 0.090491 \\
\hline 17 & $\mathrm{H}$ & 2.483314 & 1.779850 & 1.724280 \\
\hline
\end{tabular}


transition state of $\mathrm{C}-\mathrm{N}$ cleavage of

\begin{tabular}{ccccc} 
Center & Atomic & \multicolumn{3}{c}{ Coordinates (Angstroms) } \\
Number & Number & X & Y & Z \\
-------------1 & \\
1 & N & 0.000000 & 0.000000 & 0.000000 \\
2 & $\mathrm{C}$ & 0.000000 & 0.000000 & 1.439548 \\
3 & $\mathrm{C}$ & 1.441209 & 0.000000 & 1.034508 \\
4 & $\mathrm{C}$ & 2.150170 & -1.211056 & 0.916351 \\
5 & $\mathrm{H}$ & 1.633024 & -2.164204 & 0.949615 \\
6 & $\mathrm{H}$ & 3.209099 & -1.218276 & 0.678313 \\
7 & $\mathrm{C}$ & 2.218910 & 1.310560 & 1.093489 \\
8 & $\mathrm{H}$ & -0.387882 & -0.920261 & 1.884391 \\
9 & $\mathrm{H}$ & -0.352168 & 0.890018 & 1.972778 \\
10 & $\mathrm{C}$ & -0.308205 & 1.267961 & -0.664153 \\
11 & $\mathrm{H}$ & -1.401094 & 1.401976 & -0.605476 \\
12 & $\mathrm{H}$ & -0.064195 & 1.127387 & -1.725042 \\
13 & $\mathrm{C}$ & 0.396486 & 2.543917 & -0.169266 \\
14 & $\mathrm{C}$ & 1.901396 & 2.312536 & -0.029633 \\
15 & $\mathrm{H}$ & 3.289827 & 1.078896 & 1.077672 \\
16 & $\mathrm{H}$ & 2.021654 & 1.794525 & 2.060984 \\
17 & $\mathrm{H}$ & 2.298490 & 1.935210 & -0.982237 \\
18 & $\mathrm{H}$ & 2.419421 & 3.256253 & 0.180422 \\
19 & $\mathrm{H}$ & 0.193077 & 3.345989 & -0.889654 \\
20 & $\mathrm{H}$ & -0.019377 & 2.879230 & 0.791256
\end{tabular}

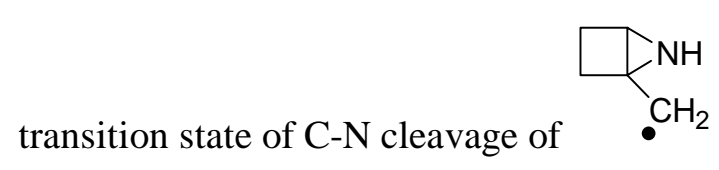

\begin{tabular}{ccccc} 
Center & Atomic & \multicolumn{4}{c}{ Coordinates (Angstroms) } \\
Number & Number & X & Y r & Z \\
---1 & N & 0.000000 & 0.000000 & 0.000000 \\
1 & C & 0.000000 & 0.000000 & 1.433392 \\
2 & $\mathrm{C}$ & 1.391725 & 0.000000 & 0.409158 \\
3 & $\mathrm{C}$ & 2.261147 & 1.032200 & 0.051115 \\
4 & $\mathrm{H}$ & 1.894620 & 1.895650 & -0.491821 \\
5 & $\mathrm{H}$ & 3.291227 & 1.028590 & 0.391806 \\
6 & $\mathrm{C}$ & 1.758352 & -1.322169 & 1.105646 \\
7 & $\mathrm{C}$ & 0.509743 & -1.300244 & 2.032232 \\
8 & $\mathrm{H}$ & -0.437026 & 0.842712 & 1.958446 \\
9 & & & &
\end{tabular}




$\begin{array}{llrrr}10 & \mathrm{H} & -0.291862 & -0.897672 & -0.400809 \\ 11 & \mathrm{H} & 0.776146 & -1.204207 & 3.088871 \\ 12 & \mathrm{H} & -0.177006 & -2.153909 & 1.929392 \\ 13 & \mathrm{H} & 2.693710 & -1.253035 & 1.668046 \\ 14 & \mathrm{H} & 1.833008 & -2.174939 & 0.416980 \\ & & & \end{array}$

\begin{tabular}{crrrr} 
Center & Atomic & \multicolumn{3}{c}{ Coordinates (Angstroms) } \\
Number & Number & X & Y & Z \\
--1 & N & 0.000000 & 0.000000 & 0.000000 \\
1 & $\mathrm{C}$ & 0.000000 & 0.000000 & 1.438803 \\
2 & $\mathrm{C}$ & 1.432276 & 0.000000 & 0.252008 \\
3 & $\mathrm{C}$ & 2.234264 & 1.106517 & 0.077988 \\
4 & $\mathrm{H}$ & 1.821498 & 2.076389 & -0.185062 \\
5 & $\mathrm{H}$ & 3.306992 & 1.041670 & 0.225022 \\
6 & $\mathrm{C}$ & 1.978027 & -1.401633 & 0.489807 \\
7 & $\mathrm{C}$ & 0.066440 & -1.344994 & 2.094379 \\
8 & $\mathrm{H}$ & -0.159334 & 0.923886 & 1.986758 \\
9 & $\mathrm{H}$ & -0.294882 & 0.902512 & -0.370543 \\
10 & $\mathrm{H}$ & -0.931872 & -1.776211 & 2.277087 \\
11 & $\mathrm{H}$ & 0.551376 & -1.244803 & 3.074518 \\
12 & $\mathrm{H}$ & 1.302492 & -3.121114 & 1.683406 \\
13 & $\mathrm{C}$ & 0.881912 & -2.260793 & 1.152131 \\
14 & $\mathrm{H}$ & 0.216850 & -2.649025 & 0.375641 \\
15 & $\mathrm{H}$ & 2.865790 & -1.327723 & 1.129385 \\
16 & $\mathrm{H}$ & 2.298323 & -1.852582 & -0.458364 \\
17 & & & &
\end{tabular}

transition state of C-N cleavage of

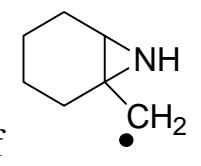

\begin{tabular}{lcccc} 
Center & Atomic & \multicolumn{3}{c}{ Coordinates (Angstroms) } \\
Number & Number & X & Y & Z \\
\hdashline 1 & N & 0.000000 & 0.000000 & 0.000000 \\
2 & $\mathrm{C}$ & 0.000000 & 0.000000 & 1.431947 \\
3 & $\mathrm{C}$ & 1.436692 & 0.000000 & 0.219973 \\
4 & $\mathrm{C}$ & 2.179028 & 1.164878 & 0.158731 \\
5 & $\mathrm{H}$ & 1.701439 & 2.139335 & 0.096346 \\
6 & $\mathrm{H}$ & 3.261229 & 1.144245 & 0.222996 \\
& & & &
\end{tabular}




\begin{tabular}{|c|c|c|c|c|}
\hline 7 & $\mathrm{C}$ & 2.101149 & -1.366443 & 0.138833 \\
\hline 8 & $\mathrm{C}$ & -0.345792 & -1.261407 & 2.164461 \\
\hline 9 & $\mathrm{H}$ & -0.065150 & 0.953940 & 1.952766 \\
\hline 10 & $\mathrm{H}$ & -0.304490 & 0.894281 & -0.384620 \\
\hline 11 & $\mathrm{H}$ & -1.443211 & -1.386589 & 2.196040 \\
\hline 12 & $\mathrm{H}$ & -0.017403 & -1.157997 & 3.207603 \\
\hline 13 & $\mathrm{C}$ & 0.287046 & -2.520962 & 1.545102 \\
\hline 14 & $\mathrm{H}$ & 0.119246 & -3.368102 & 2.221658 \\
\hline 15 & $\mathrm{H}$ & -0.218988 & -2.760656 & 0.601881 \\
\hline 16 & $\mathrm{C}$ & 1.787888 & -2.340175 & 1.289672 \\
\hline 17 & $\mathrm{H}$ & 2.270447 & -1.981651 & 2.210184 \\
\hline 18 & $\mathrm{H}$ & 2.244849 & -3.309696 & 1.053423 \\
\hline 19 & $\mathrm{H}$ & 3.186013 & -1.221382 & 0.080803 \\
\hline 20 & $\mathrm{H}$ & 1.787155 & -1.825444 & -0.809013 \\
\hline
\end{tabular}

\begin{tabular}{|c|c|c|c|c|}
\hline \multirow{2}{*}{$\begin{array}{l}\text { Center } \\
\text { Number }\end{array}$} & \multirow{2}{*}{$\begin{array}{l}\text { Atomic } \\
\text { Number }\end{array}$} & \multicolumn{3}{|c|}{ Coordinates (Angstroms) } \\
\hline & & $X$ & Y & $\mathrm{Z}$ \\
\hline 1 & $\mathrm{~N}$ & 0.000000 & 0.000000 & 0.000000 \\
\hline 2 & $\mathrm{C}$ & 0.000000 & 0.000000 & 1.454820 \\
\hline 3 & $\mathrm{C}$ & 1.429684 & 0.000000 & 0.228589 \\
\hline 4 & $\mathrm{C}$ & 2.304215 & -1.054678 & 0.180508 \\
\hline 5 & $\mathrm{C}$ & -0.560340 & -1.156248 & 0.763611 \\
\hline 6 & $\mathrm{H}$ & 1.985305 & -2.085197 & 0.074734 \\
\hline 7 & $\mathrm{H}$ & 3.367177 & -0.871505 & 0.291001 \\
\hline 8 & $\mathrm{H}$ & 1.823560 & 1.014177 & 0.238476 \\
\hline 9 & $\mathrm{H}$ & -0.414809 & 0.800692 & 2.062768 \\
\hline 10 & $\mathrm{H}$ & -0.043442 & -2.113018 & 0.768275 \\
\hline 11 & $\mathrm{H}$ & -1.640067 & -1.223513 & 0.647776 \\
\hline
\end{tabular}

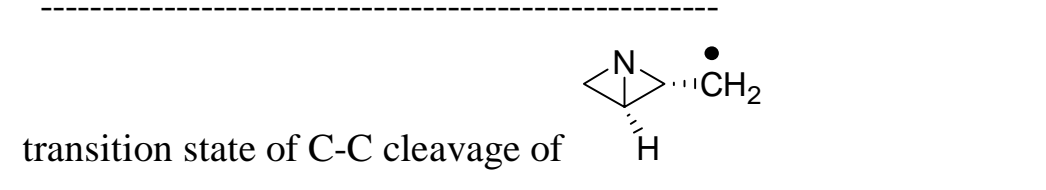

\begin{tabular}{ccccc} 
Center & Atomic & \multicolumn{3}{c}{ Coordinates (Angstroms) } \\
Number & Number & X & Y & Z \\
- \hdashline 1 & N & 0.000000 & 0.000000 & 0.000000 \\
2 & C & 0.000000 & 0.000000 & 1.453719 \\
3 & C & 1.437008 & 0.000000 & 0.197612
\end{tabular}




\begin{tabular}{|c|c|c|c|c|}
\hline 4 & $\mathrm{C}$ & 2.191599 & -1.110964 & -0.055238 \\
\hline 5 & $\mathrm{C}$ & -0.513569 & 1.174702 & 0.772361 \\
\hline 6 & $\mathrm{H}$ & 3.268138 & -1.081420 & 0.067589 \\
\hline 7 & $\mathrm{H}$ & 1.730833 & -2.047437 & -0.351903 \\
\hline 8 & $\mathrm{H}$ & 1.917462 & 0.956572 & 0.402962 \\
\hline 9 & $\mathrm{H}$ & -0.399637 & -0.805134 & 2.060511 \\
\hline 10 & $\mathrm{H}$ & 0.060412 & 2.103233 & 0.760926 \\
\hline 11 & $\mathrm{H}$ & -1.587761 & 1.298702 & 0.654074 \\
\hline \multicolumn{5}{|c|}{ transition state of $\mathrm{C}-\mathrm{C}$ cleavage of } \\
\hline Center & Atomic & \multicolumn{3}{|c|}{ Coordinates (Angstroms) } \\
\hline Number & Number & $X$ & $\mathrm{Y}$ & $\mathrm{Z}$ \\
\hline 1 & $\mathrm{~N}$ & 0.000000 & 0.000000 & 0.000000 \\
\hline 2 & $\mathrm{C}$ & 0.000000 & 0.000000 & 1.465874 \\
\hline 3 & $\mathrm{C}$ & 1.424187 & 0.000000 & 0.275312 \\
\hline 4 & $\mathrm{C}$ & 2.330434 & -1.028154 & 0.176948 \\
\hline 5 & $\mathrm{H}$ & 2.053172 & -2.055671 & -0.027789 \\
\hline 6 & $\mathrm{H}$ & 3.382051 & -0.823674 & 0.342755 \\
\hline 7 & $\mathrm{H}$ & 1.798000 & 1.017492 & 0.370133 \\
\hline 8 & $\mathrm{H}$ & -0.146779 & 0.897602 & 2.061435 \\
\hline 9 & $\mathrm{C}$ & -0.551074 & -1.399843 & 1.598392 \\
\hline 10 & $\mathrm{C}$ & -0.583860 & -1.389851 & 0.042815 \\
\hline 11 & $\mathrm{H}$ & -0.004396 & -2.146963 & -0.490143 \\
\hline 12 & $\mathrm{H}$ & -1.594273 & -1.354929 & -0.370987 \\
\hline 13 & $\mathrm{H}$ & 0.109934 & -2.136876 & 2.070075 \\
\hline 14 & $\mathrm{H}$ & -1.537155 & -1.465152 & 2.071014 \\
\hline
\end{tabular}

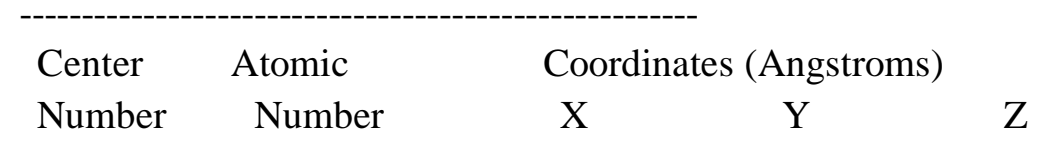

$\begin{array}{lllll}1 & \mathrm{~N} & 0.000000 & 0.000000 & 0.000000 \\ 2 & \mathrm{C} & 0.000000 & 0.000000 & 1.457638 \\ 3 & \mathrm{C} & 1.427855 & 0.000000 & 0.261520 \\ 4 & \mathrm{C} & 2.192768 & -1.126742 & 0.073566 \\ 5 & \mathrm{H} & 1.741435 & -2.059614 & -0.246924 \\ 6 & \mathrm{H} & 3.249264 & -1.120694 & 0.318199 \\ 7 & \mathrm{H} & 1.905701 & 0.961985 & 0.452814\end{array}$




$\begin{array}{rrrrr}8 & \mathrm{H} & -0.126736 & -0.900746 & 2.049955 \\ 9 & \mathrm{C} & -0.543536 & 1.402626 & 1.600680 \\ 10 & \mathrm{C} & -0.537680 & 1.407641 & 0.042342 \\ 11 & \mathrm{H} & 0.106744 & 2.139831 & -0.454287 \\ 12 & \mathrm{H} & -1.533254 & 1.437437 & -0.406763 \\ 13 & \mathrm{H} & 0.102140 & 2.137930 & 2.098217 \\ 14 & \mathrm{H} & -1.541342 & 1.469861 & 2.051193 \\ - & & & \\ \text { transition state of C-C cleavage of } & & \end{array}$

\begin{tabular}{|c|c|c|c|c|}
\hline \multirow{2}{*}{$\begin{array}{l}\text { Center } \\
\text { Number }\end{array}$} & \multirow{2}{*}{$\begin{array}{l}\text { Atomic } \\
\text { Number }\end{array}$} & \multicolumn{3}{|c|}{ Coordinates (Angstroms) } \\
\hline & & $\mathrm{X}$ & $\mathrm{Y}$ & $\mathrm{Z}$ \\
\hline 1 & $\mathrm{~N}$ & 0.000000 & 0.000000 & 0.000000 \\
\hline 2 & $\mathrm{C}$ & 0.000000 & 0.000000 & 1.438230 \\
\hline 3 & $\mathrm{C}$ & 1.426091 & 0.000000 & 0.250894 \\
\hline 4 & $\mathrm{C}$ & 2.330028 & -1.027467 & 0.091888 \\
\hline 5 & $\mathrm{H}$ & 2.049155 & -2.041918 & -0.164158 \\
\hline 6 & $\mathrm{H}$ & 3.386833 & -0.825207 & 0.226624 \\
\hline 7 & $\mathrm{H}$ & 1.816180 & 1.011246 & 0.350086 \\
\hline 8 & $\mathrm{H}$ & -0.111989 & 0.950457 & 1.952936 \\
\hline 9 & $\mathrm{C}$ & -0.459721 & -1.312829 & 2.016533 \\
\hline 10 & $\mathrm{C}$ & -0.599729 & -1.280342 & -0.425249 \\
\hline 11 & $\mathrm{H}$ & -0.077720 & -1.664757 & -1.306286 \\
\hline 12 & $\mathrm{H}$ & -1.632886 & -1.058968 & -0.714983 \\
\hline 13 & $\mathrm{H}$ & 0.287688 & -2.921544 & 0.741662 \\
\hline 14 & $\mathrm{H}$ & -1.470700 & -2.874182 & 0.821440 \\
\hline 15 & $\mathrm{H}$ & 0.222447 & -1.709119 & 2.779349 \\
\hline 16 & $\mathrm{H}$ & -1.434114 & -1.177555 & 2.508642 \\
\hline 17 & $\mathrm{C}$ & -0.572855 & -2.249399 & 0.787169 \\
\hline
\end{tabular}

\begin{tabular}{ccccc}
$\begin{array}{c}\text { Center } \\
\text { Number }\end{array}$ & $\begin{array}{c}\text { Atomic } \\
\text { Number }\end{array}$ & \multicolumn{3}{c}{ Coordinates (Angstroms) } \\
$-\mathrm{X}$ & $\mathrm{Y}$ & $\mathrm{Z}$ \\
\hline 1 & $\mathrm{~N}$ & 0.000000 & 0.000000 & 0.000000 \\
2 & $\mathrm{C}$ & 0.000000 & 0.000000 & 1.432810 \\
3 & $\mathrm{C}$ & 1.429804 & 0.000000 & 0.224310 \\
4 & $\mathrm{C}$ & 2.197322 & -1.119977 & -0.008663 \\
5 & $\mathrm{H}$ & 1.738485 & -2.050266 & -0.326244
\end{tabular}




\begin{tabular}{|c|c|c|c|c|}
\hline 6 & $\mathrm{H}$ & 3.261093 & -1.113748 & 0.204047 \\
\hline 7 & $\mathrm{H}$ & 1.914771 & 0.956113 & 0.426089 \\
\hline 8 & $\mathrm{C}$ & -0.420547 & 1.331213 & 2.008254 \\
\hline 9 & $\mathrm{H}$ & -0.114094 & -0.948933 & 1.945503 \\
\hline 10 & $\mathrm{C}$ & -0.563712 & 1.298867 & -0.418931 \\
\hline 11 & $\mathrm{H}$ & -0.074573 & 1.644070 & -1.334747 \\
\hline 12 & $\mathrm{H}$ & -1.624193 & 1.131970 & -0.641389 \\
\hline 13 & $\mathrm{C}$ & -0.408612 & 2.271149 & 0.777879 \\
\hline 14 & $\mathrm{H}$ & -1.206663 & 3.018876 & 0.806679 \\
\hline 15 & $\mathrm{H}$ & 0.540084 & 2.815495 & 0.721277 \\
\hline 16 & $\mathrm{H}$ & 0.230370 & 1.684484 & 2.817852 \\
\hline 17 & $\mathrm{H}$ & -1.432217 & 1.250667 & 2.436336 \\
\hline
\end{tabular}

\begin{tabular}{|c|c|c|c|c|}
\hline \multirow{2}{*}{$\begin{array}{l}\text { Center } \\
\text { Number }\end{array}$} & \multirow{2}{*}{$\begin{array}{l}\text { Atomic } \\
\text { Number }\end{array}$} & \multicolumn{3}{|c|}{ Coordinates (Angstroms) } \\
\hline & & $X$ & Y & $\mathrm{Z}$ \\
\hline 1 & $\mathrm{~N}$ & 0.000000 & 0.000000 & 0.000000 \\
\hline 2 & $\mathrm{C}$ & 0.000000 & 0.000000 & 1.426469 \\
\hline 3 & $\mathrm{C}$ & 1.425227 & 0.000000 & 0.222089 \\
\hline 4 & $\mathrm{C}$ & 2.304305 & -1.054595 & 0.111445 \\
\hline 5 & $\mathrm{H}$ & 1.993570 & -2.074669 & -0.084319 \\
\hline 6 & $\mathrm{H}$ & 3.367299 & -0.873892 & 0.226086 \\
\hline 7 & $\mathrm{H}$ & 1.837034 & 1.006327 & 0.288108 \\
\hline 8 & $\mathrm{H}$ & -0.077185 & 0.982486 & 1.890111 \\
\hline 9 & $\mathrm{C}$ & -0.317946 & -1.213749 & 2.259838 \\
\hline 10 & $\mathrm{C}$ & -0.649056 & -1.107883 & -0.728381 \\
\hline 11 & $\mathrm{C}$ & -1.487114 & -2.016678 & 0.179949 \\
\hline 12 & $\mathrm{C}$ & -0.681898 & -2.446538 & 1.411011 \\
\hline 13 & $\mathrm{H}$ & 0.511404 & -1.445651 & 2.945177 \\
\hline 14 & $\mathrm{H}$ & -1.172641 & -0.959852 & 2.905877 \\
\hline 15 & $\mathrm{H}$ & 0.228750 & -2.965371 & 1.087195 \\
\hline 16 & $\mathrm{H}$ & -1.248566 & -3.160832 & 2.019708 \\
\hline 17 & $\mathrm{H}$ & -2.391642 & -1.483119 & 0.504544 \\
\hline 18 & $\mathrm{H}$ & -1.824645 & -2.885541 & -0.398066 \\
\hline 19 & $\mathrm{H}$ & 0.115513 & -1.701628 & -1.242221 \\
\hline 20 & $\mathrm{H}$ & -1.285026 & -0.667354 & -1.507445 \\
\hline
\end{tabular}




\begin{tabular}{|c|c|c|c|c|}
\hline \multirow{2}{*}{$\begin{array}{l}\text { Center } \\
\text { Number }\end{array}$} & \multirow{2}{*}{$\begin{array}{l}\text { Atomic } \\
\text { Number }\end{array}$} & \multicolumn{3}{|c|}{ Coordinates (Angstroms) } \\
\hline & & $\mathrm{X}$ & $\mathrm{Y}$ & $\mathrm{Z}$ \\
\hline 1 & $\mathrm{~N}$ & 0.000000 & 0.000000 & 0.000000 \\
\hline 2 & $\mathrm{C}$ & 0.000000 & 0.000000 & 1.425784 \\
\hline 3 & $\mathrm{C}$ & 1.418044 & 0.000000 & 0.227870 \\
\hline 4 & $\mathrm{C}$ & 2.195204 & -1.111979 & -0.042070 \\
\hline 5 & $\mathrm{H}$ & 1.739498 & -2.034488 & -0.386183 \\
\hline 6 & $\mathrm{H}$ & 3.258996 & -1.108187 & 0.172153 \\
\hline 7 & $\mathrm{H}$ & 1.905770 & 0.951082 & 0.454801 \\
\hline 8 & $\mathrm{H}$ & -0.114670 & -0.975841 & 1.888726 \\
\hline 9 & $\mathrm{C}$ & -0.229957 & 1.253798 & 2.239085 \\
\hline 10 & $\mathrm{C}$ & -0.615843 & 1.135612 & -0.696899 \\
\hline 11 & $\mathrm{H}$ & -0.144481 & 1.207511 & -1.682960 \\
\hline 12 & $\mathrm{H}$ & -1.670068 & 0.869443 & -0.856667 \\
\hline 13 & $\mathrm{C}$ & -0.558411 & 2.475803 & 0.054966 \\
\hline 14 & $\mathrm{H}$ & 0.471188 & 2.859990 & 0.081633 \\
\hline 15 & $\mathrm{H}$ & -1.154948 & 3.218432 & -0.489420 \\
\hline 16 & $\mathrm{C}$ & -1.082857 & 2.294129 & 1.485740 \\
\hline 17 & $\mathrm{H}$ & -2.127540 & 1.954608 & 1.445364 \\
\hline 18 & $\mathrm{H}$ & -1.078080 & 3.246416 & 2.029512 \\
\hline 19 & $\mathrm{H}$ & 0.731407 & 1.721868 & 2.507077 \\
\hline 20 & $\mathrm{H}$ & -0.702044 & 0.981352 & 3.190667 \\
\hline
\end{tabular}

\begin{tabular}{ccrcc} 
Center & Atomic & \multicolumn{3}{c}{ Coordinates (Angstroms) } \\
Number & Number & X & Y & Z \\
-1 & N & 0.000000 & 0.000000 & 0.000000 \\
2 & C & 0.000000 & 0.000000 & 1.428916 \\
3 & $\mathrm{C}$ & 1.443537 & 0.000000 & 0.203081 \\
4 & $\mathrm{C}$ & 2.204563 & -1.143944 & 0.134446 \\
5 & $\mathrm{H}$ & 1.738457 & -2.116876 & 0.024004 \\
6 & $\mathrm{H}$ & 3.277665 & -1.103631 & 0.289724 \\
7 & $\mathrm{C}$ & 1.992027 & 1.427534 & 0.116378 \\
8 & $\mathrm{H}$ & -0.137547 & -0.954867 & 1.921464 \\
9 & $\mathrm{H}$ & -0.139053 & 0.915768 & 1.998646 \\
10 & $\mathrm{C}$ & -0.331216 & 1.309981 & -0.588340 \\
11 & $\mathrm{C}$ & 0.745302 & 2.313745 & -0.102178 \\
12 & $\mathrm{H}$ & 0.437999 & 2.794360 & 0.832616 \\
13 & $\mathrm{H}$ & 0.917432 & 3.112338 & -0.829854 \\
14 & $\mathrm{H}$ & 2.671531 & 1.485703 & -0.742063
\end{tabular}




\begin{tabular}{|c|c|c|c|c|}
\hline 15 & $\mathrm{H}$ & 2.567459 & 1.720909 & 1.000226 \\
\hline 16 & $\mathrm{H}$ & -0.289293 & 1.196859 & -1.677593 \\
\hline 17 & $\mathrm{H}$ & -1.351965 & 1.591972 & -0.311433 \\
\hline \multicolumn{5}{|c|}{ transition state of C-C cleavage of } \\
\hline \multirow{2}{*}{$\begin{array}{l}\text { Center } \\
\text { Number }\end{array}$} & \multirow{2}{*}{$\begin{array}{l}\text { Atomic } \\
\text { Number }\end{array}$} & \multicolumn{3}{|c|}{ Coordinates (Angstroms) } \\
\hline & & $X$ & Y & $\mathrm{Z}$ \\
\hline 1 & $\mathrm{~N}$ & 0.000000 & 0.000000 & 0.000000 \\
\hline 2 & $\mathrm{C}$ & 0.000000 & 0.000000 & 1.422038 \\
\hline 3 & $\mathrm{C}$ & 1.436935 & 0.000000 & 0.215623 \\
\hline 4 & $\mathrm{C}$ & 2.149576 & -1.180120 & 0.102014 \\
\hline 5 & $\mathrm{H}$ & 1.636014 & -2.123206 & -0.052121 \\
\hline 6 & $\mathrm{H}$ & 3.218867 & -1.200227 & 0.285300 \\
\hline 7 & $\mathrm{C}$ & 2.106966 & 1.361738 & 0.139771 \\
\hline 8 & $\mathrm{H}$ & -0.180658 & -0.939734 & 1.931796 \\
\hline 9 & $\mathrm{H}$ & -0.109794 & 0.932807 & 1.975782 \\
\hline 10 & $\mathrm{C}$ & -0.512328 & 1.202625 & -0.681239 \\
\hline 11 & $\mathrm{H}$ & -0.623325 & 2.049506 & 0.018577 \\
\hline 12 & $\mathrm{H}$ & -1.512897 & 0.975506 & -1.067013 \\
\hline 13 & $\mathrm{C}$ & 0.449404 & 1.579750 & -1.810793 \\
\hline 14 & $\mathrm{C}$ & 1.839486 & 1.987370 & -1.256820 \\
\hline 15 & $\mathrm{H}$ & 1.913282 & 3.078933 & -1.177318 \\
\hline 16 & $\mathrm{H}$ & 0.019551 & 2.385549 & -2.416012 \\
\hline 17 & $\mathrm{H}$ & 0.550203 & 0.707604 & -2.467895 \\
\hline 18 & $\mathrm{H}$ & 2.617630 & 1.670707 & -1.960025 \\
\hline 19 & $\mathrm{H}$ & 3.181811 & 1.270155 & 0.324289 \\
\hline 20 & $\mathrm{H}$ & 1.713461 & 2.028024 & 0.914745 \\
\hline
\end{tabular}

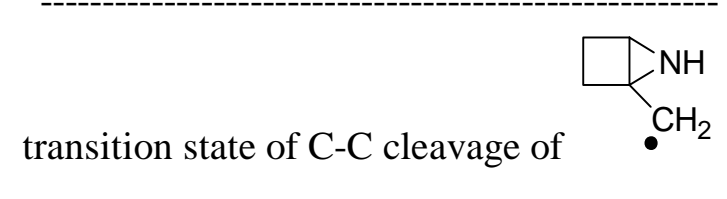

\begin{tabular}{ccccc} 
Center & Atomic & \multicolumn{3}{c}{ Coordinates (Angstroms) } \\
Number & Number & X & Y & Z \\
\hdashline 1 & N & 0.000000 & 0.000000 & 0.000000 \\
2 & C & 0.000000 & 0.000000 & 1.440210 \\
3 & C & 1.467262 & 0.000000 & 1.088845 \\
4 & C & 2.396463 & 0.984367 & 0.807896 \\
5 & H & 2.087839 & 2.008280 & 0.627720
\end{tabular}




\begin{tabular}{|c|c|c|c|c|}
\hline 6 & $\mathrm{H}$ & 3.446478 & 0.737996 & 0.683262 \\
\hline 7 & $\mathrm{C}$ & 1.611615 & -1.457315 & 1.538285 \\
\hline 8 & $\mathrm{C}$ & 0.146308 & -1.392167 & 2.087702 \\
\hline 9 & $\mathrm{H}$ & -0.541814 & 0.823845 & 1.908806 \\
\hline 10 & $\mathrm{H}$ & -0.017756 & -0.969706 & -0.338693 \\
\hline 11 & $\mathrm{H}$ & -0.559782 & -2.152936 & 1.736079 \\
\hline 12 & $\mathrm{H}$ & 0.119043 & -1.359962 & 3.181087 \\
\hline 13 & $\mathrm{H}$ & 2.367736 & -1.595036 & 2.317894 \\
\hline 14 & $\mathrm{H}$ & 1.804122 & -2.188520 & 0.745058 \\
\hline
\end{tabular}

\begin{tabular}{crrrr} 
Center & Atomic & \multicolumn{3}{c}{ Coordinates (Angstroms) } \\
Number & Number & X & Y & Z \\
-1 & N & 0.000000 & 0.000000 & 0.000000 \\
2 & $\mathrm{C}$ & 0.000000 & 0.000000 & 1.449596 \\
3 & $\mathrm{C}$ & 1.459676 & 0.000000 & 1.099886 \\
4 & $\mathrm{C}$ & 2.253997 & 1.134876 & 1.001365 \\
5 & $\mathrm{H}$ & 1.831830 & 2.130790 & 1.101898 \\
6 & $\mathrm{H}$ & 3.311057 & 1.063280 & 0.765313 \\
7 & $\mathrm{C}$ & 1.972460 & -1.430228 & 1.146185 \\
8 & $\mathrm{C}$ & -0.316344 & -1.379309 & 2.022027 \\
9 & $\mathrm{H}$ & -0.419030 & 0.861706 & 1.978036 \\
10 & $\mathrm{H}$ & -0.036223 & 0.978514 & -0.309243 \\
11 & $\mathrm{C}$ & 0.715450 & -2.309516 & 1.345194 \\
12 & $\mathrm{H}$ & 0.334476 & -2.629301 & 0.372422 \\
13 & $\mathrm{H}$ & 0.922338 & -3.206696 & 1.937106 \\
14 & $\mathrm{H}$ & 2.650563 & -1.518570 & 2.005904 \\
15 & $\mathrm{H}$ & 2.542776 & -1.703755 & 0.252572 \\
16 & $\mathrm{H}$ & -0.173463 & -1.354220 & 3.110230 \\
17 & $\mathrm{H}$ & -1.351019 & -1.679807 & 1.823607 \\
& & & &
\end{tabular}

transition state of $\mathrm{C}-\mathrm{C}$ cleavage of

\begin{tabular}{|c|c|c|c|c|}
\hline \multirow{2}{*}{$\begin{array}{l}\text { Center } \\
\text { Number }\end{array}$} & \multirow{2}{*}{$\begin{array}{l}\text { Atomic } \\
\text { Number }\end{array}$} & \multicolumn{3}{|c|}{ Coordinates (Angstroms) } \\
\hline & & $\mathrm{X}$ & Y & $\mathrm{Z}$ \\
\hline 1 & $\mathrm{~N}$ & 0.000000 & 0.000000 & 0.000000 \\
\hline
\end{tabular}




$\begin{array}{rrrrr}2 & \mathrm{C} & 0.000000 & 0.000000 & 1.454097 \\ 3 & \mathrm{C} & 1.469116 & 0.000000 & 1.133079 \\ 4 & \mathrm{C} & 2.177299 & 1.193926 & 1.119173 \\ 5 & \mathrm{H} & 1.693694 & 2.141707 & 1.339668 \\ 6 & \mathrm{H} & 3.229345 & 1.222038 & 0.850828 \\ 7 & \mathrm{C} & 2.188188 & -1.327399 & 0.958951 \\ 8 & \mathrm{C} & -0.635103 & -1.248998 & 2.084093 \\ 9 & \mathrm{H} & -0.343477 & 0.921526 & 1.938454 \\ 10 & \mathrm{H} & -0.033527 & 0.978706 & -0.309800 \\ 11 & \mathrm{C} & 1.249751 & -2.541113 & 0.957943 \\ 12 & \mathrm{H} & 0.674695 & -2.560645 & 0.025834 \\ 13 & \mathrm{H} & 1.847646 & -3.459954 & 0.996508 \\ 14 & \mathrm{H} & 2.894764 & -1.428991 & 1.796169 \\ 15 & \mathrm{H} & 2.791317 & -1.301841 & 0.043583 \\ 16 & \mathrm{C} & 0.301055 & -2.470999 & 2.159771 \\ 17 & \mathrm{H} & -0.297541 & -3.386387 & 2.238483 \\ 18 & \mathrm{H} & 0.903881 & -2.415296 & 3.077854 \\ 19 & \mathrm{H} & -1.519709 & -1.491084 & 1.482374 \\ 20 & \mathrm{H} & -0.995480 & -0.998454 & 3.089168\end{array}$

Table S12. $\quad \hat{\mathrm{S}}^{2}$ values of all the radicals in Table S1-S3

\begin{tabular}{ccccc}
\hline $\mathrm{R}_{2}{ }^{\mathrm{b}}$ & $\mathrm{R}_{1}$ & Reactants & $\begin{array}{c}\text { Transition States } \\
\text { of C-C cleavage }\end{array}$ & $\begin{array}{c}\text { Transition States } \\
\text { of C-N cleavage }\end{array}$ \\
\hline $\mathrm{H}$ & cis $-\mathrm{H}$ & 0.7563 & 0.7759 & 0.7775 \\
$\mathrm{H}$ & trans $-\mathrm{CH}_{2} \mathrm{CH}_{3}$ & 0.7552 & 0.7782 & 0.7756 \\
$\mathrm{H}$ & trans $-\mathrm{COCH}_{3}$ & 0.7552 & 0.7788 & 0.7723 \\
$\mathrm{H}$ & trans $-\mathrm{CONH}_{2}$ & 0.7552 & 0.7787 & 0.7726 \\
$\mathrm{H}$ & trans $-\mathrm{COOCH} 3$ & 0.7552 & 0.7789 & 0.7719 \\
cis $-\mathrm{CH}_{3}$ & cis $-\mathrm{H}$ & 0.7565 & 0.7753 & 0.7775 \\
trans $-\mathrm{CH}_{3}$ & cis $-\mathrm{H}$ & 0.7563 & 0.7762 & 0.7779 \\
cis $-\mathrm{CHO}$ & cis $-\mathrm{H}$ & 0.7567 & 0.7691 & 0.7788 \\
trans $-\mathrm{CHO}$ & trans $-\mathrm{H}$ & 0.7562 & 0.7709 & 0.779 \\
cis $-\mathrm{COCH}{ }_{3}$ & cis $-\mathrm{H}$ & 0.7565 & 0.7708 & 0.7787 \\
trans $-\mathrm{COCH}$ & trans $-\mathrm{H}$ & 0.7559 & 0.7721 & 0.7809 \\
cis $-\mathrm{COOH}$ & cis $-\mathrm{H}$ & 0.7563 & 0.7728 & 0.7786 \\
trans $-\mathrm{COOH}$ & trans $-\mathrm{H}$ & 0.7556 & 0.7744 & 0.7806 \\
cis $-\mathrm{CONH}{ }_{2}$ & cis $-\mathrm{H}$ & 0.7563 & 0.7736 & 0.7786 \\
trans $-\mathrm{CONH}{ }_{2}$ & cis $-\mathrm{H}$ & 0.7561 & 0.7745 & 0.7785 \\
cis $-\mathrm{CH}=\mathrm{CH}_{2}$ & cis $-\mathrm{H}$ & 0.7563 & 0.7697 & 0.7785 \\
trans $-\mathrm{CH}=\mathrm{CH}_{2}$ & cis $-\mathrm{H}$ & 0.7561 & 0.7683 & 0.7788
\end{tabular}




\begin{tabular}{ccccc} 
cis $-\mathrm{C} \equiv \mathrm{CH}$ & cis $-\mathrm{H}$ & 0.7563 & 0.7709 & 0.7785 \\
trans $-\mathrm{C} \equiv \mathrm{CH}$ & trans $-\mathrm{H}$ & 0.7555 & 0.7706 & 0.7800 \\
cis $-\mathrm{Ph}$ & trans $-\mathrm{H}$ & 0.7565 & 0.7701 & 0.7780 \\
$\mathrm{Ph}$ & cis $-\mathrm{H}$ & 0.7562 & 0.7704 & 0.7788 \\
$\mathrm{H}$ & trans $-\mathrm{CH}_{3}$ & 0.7552 & 0.7782 & 0.7755 \\
cis $-\mathrm{CH}_{3}$ & trans $-\mathrm{CH}_{3}$ & 0.7564 & 0.7753 & 0.7741 \\
trans $-\mathrm{CH}{ }_{3}$ & cis $-\mathrm{CH}_{3}$ & 0.7563 & 0.7762 & 0.7741 \\
cis $-\mathrm{CHO}$ & trans $-\mathrm{CH}_{3}$ & 0.7569 & 0.7688 & 0.7768 \\
trans $-\mathrm{CHO}$ & cis $-\mathrm{CH}_{3}$ & 0.7565 & 0.7694 & 0.7764 \\
cis $-\mathrm{Ph}$ & trans $-\mathrm{CH}$ & 0.7564 & 0.7697 & 0.7751 \\
trans $-\mathrm{Ph}$ & cis $-\mathrm{CH}$ & 0.7564 & 0.7691 & 0.7758 \\
$\mathrm{H}$ & cis $-\mathrm{CHO}$ & 0.7562 & 0.7773 & 0.7737 \\
cis $-\mathrm{CH} H_{3}$ & trans $-\mathrm{CHO}$ & 0.7564 & 0.7764 & 0.7773 \\
trans $-\mathrm{CH}$ & cis $-\mathrm{CHO}$ & 0.7562 & 0.7776 & 0.7744 \\
cis $-\mathrm{CHO}$ & trans $-\mathrm{CHO}$ & 0.7577 & 0.7675 & 0.7783 \\
trans $-\mathrm{CHO}$ & cis $-\mathrm{CHO}$ & 0.7565 & 0.7699 & 0.7750 \\
cis $-\mathrm{Ph}$ & trans $-\mathrm{CHO}$ & 0.7562 & 0.7676 & 0.7785 \\
trans $-\mathrm{Ph}$ & cis $-\mathrm{CHO}$ & 0.7565 & 0.7695 & 0.7750 \\
$\mathrm{H}$ & trans $-\mathrm{Ph}$ & 0.7551 & 0.7784 & 0.7676 \\
cis $-\mathrm{CH} \mathrm{H}_{3}$ & trans $-\mathrm{Ph}$ & 0.7562 & 0.7767 & 0.7731 \\
trans $-\mathrm{CH} H_{3}$ & cis $-\mathrm{Ph}$ & 0.7562 & 0.7772 & 0.7690 \\
cis $-\mathrm{CHO}$ & trans $-\mathrm{Ph}$ & 0.7564 & 0.7704 & 0.7739 \\
trans $-\mathrm{CHO}$ & cis $-\mathrm{Ph}$ & 0.7564 & 0.771 & 0.7713 \\
cis $-\mathrm{Ph}$ & trans $-\mathrm{Ph}$ & 0.7565 & 0.7695 & 0.7712 \\
trans $-\mathrm{Ph}$ & cis $-\mathrm{Ph}$ & 0.7563 & 0.7707 & 0.7710 \\
\hline
\end{tabular}

Table S13. Calculated Barriers $\left(\Delta H^{\dagger}\right)$ and Reaction Enthalpies $(\Delta H)$ for cis- and trans-2-Aziridinylcarbinyl Radical in TableS1 ${ }^{\mathrm{a}}$. $(\mathrm{kJ} / \mathrm{mol})$

\begin{tabular}{ccc}
\hline $\mathrm{R}_{1}$ & Calculated Barriers & Reaction Enthalpies \\
\cline { 2 - 3 } cis $-\mathrm{H}$ & 79.6 & -4.6 \\
trans $-\mathrm{CH}_{3}$ & 77.5 & 7.6 \\
trans $-\mathrm{CH}_{2} \mathrm{CH}_{3}$ & 77.5 & 8.1 \\
cis $-\mathrm{CHO}$ & 21.6 & 2.2 \\
trans $-\mathrm{COCH}$ & 26.1 & 2.1 \\
trans $-\mathrm{CONH}$ & 39.9 & 2.7 \\
trans $-\mathrm{COOCH}$ & 31.6 & 2.5 \\
trans $-\mathrm{Ph}$ & 45.5 & 5.0 \\
\hline
\end{tabular}

${ }^{\mathrm{a}} \Delta H^{+}$'s and $\Delta H_{s}$ are calculated using the $\mathrm{ONIOM}(\mathrm{QCISD}(\mathrm{T}) / 6-311+\mathrm{G}(2 \mathrm{~d}, 2 \mathrm{p}): \mathrm{B} 3 \mathrm{LYP} / 6-311+\mathrm{G}(3 \mathrm{df}, 2 \mathrm{p}))$ method. 
Table S14. Reaction free energies $\left(\Delta G, \mathrm{~kJ} \mathrm{~mol}^{-1}\right)$ for $\mathrm{N}$ - and $\mathrm{C} 1$-disubstituted 2-aziridinylmethyl radicals ${ }^{\mathrm{b}}$.

\begin{tabular}{cccc}
\hline $\mathrm{R}_{2}$ & $\mathrm{R}_{1}$ & C-N cleavage & C-C cleavage \\
\hline $\mathrm{H}$ & cis $-\mathrm{H}$ & -34.5 & -82.2 \\
$\mathrm{H}$ & trans $-\mathrm{CH}_{3}$ & -62.8 & -79.6 \\
$\mathrm{H}$ & cis $-\mathrm{CHO}$ & -36.2 & -92.5 \\
$\mathrm{H}$ & trans $-\mathrm{COCH}{ }_{3}$ & -40.3 & -71.4 \\
$\mathrm{H}$ & trans $-\mathrm{CONH}{ }_{2}$ & -45.2 & -61.4 \\
$\mathrm{H}$ & trans $-\mathrm{COOCH}$ & -42.4 & -77.9 \\
$\mathrm{H}$ & trans $-\mathrm{Ph}$ & -86.2 & -73.4 \\
cis $-\mathrm{CH}_{3}$ & cis $-\mathrm{H}$ & -29.3 & -86.6 \\
trans $-\mathrm{CH}$ & cis $-\mathrm{H}$ & -22.0 & -64.4 \\
cis $-\mathrm{CHO}$ & cis $-\mathrm{H}$ & -19.3 & -151.5 \\
trans $-\mathrm{CHO}$ & trans $-\mathrm{H}$ & -17.4 & -117.6 \\
cis $-\mathrm{Ph}$ & trans $-\mathrm{H}$ & -28.1 & -131.2 \\
trans $-\mathrm{Ph}$ & cis $-\mathrm{H}$ & -14.8 & -89.9 \\
\hline
\end{tabular}

${ }^{\mathrm{b}} \Delta G s$ are calculated using the $\mathrm{B} 3 \mathrm{LYP} / 6-311++\mathrm{G}(3 \mathrm{df}, 2 \mathrm{p}) / / \mathrm{B} 3 \mathrm{LYP} / 6-31 \mathrm{G}(\mathrm{d})$ method. 


\section{Possible explanation for the extraordinary $C_{1}$-substituent effects:}

The NBO analyses reveal a significant hyperconjugative interaction between the $\sigma\left(\mathrm{C}_{1}-\mathrm{C}_{2}\right)$ orbital and the antibonding $\pi^{*}(\mathrm{CH}=\mathrm{O})$ or $\pi^{*}\left(\mathrm{CH}=\mathrm{CH}_{2}\right)$ orbital. At the same time, there is a significant hyperconjugative interaction between the $\sigma\left(\mathrm{C}_{1}-\mathrm{N}\right)$ orbital and the antibonding $\pi^{*}(\mathrm{CH}=\mathrm{O})$ or $\pi^{*}\left(\mathrm{CH}=\mathrm{CH}_{2}\right)$ orbital. The energies of these hyperconjugation interactions can be calculated by deletion of the off-diagonal Fock matrix elements between the interacting orbitals and from the second order perturbation approach, ${ }^{14}$ i.e.

$$
E(2)=q_{i} \frac{\langle i|F| j\rangle^{2}}{\varepsilon_{j}-\varepsilon_{i}}=q_{i} \frac{F_{i j}{ }^{2}}{\varepsilon_{j}-\varepsilon_{i}}
$$

where $\langle i|F| j\rangle$, or $F_{i j}$ is the Fock matrix element between the $i$ and $j$ NBO orbitals, $\varepsilon_{i}$ and $\varepsilon_{j}$ are the energies of $i$ and $j$ NBO's, and $q_{i}$ is the population of the donor orbital. As shown in Table 5 , the hyperconjugative interaction between $\sigma\left(\mathrm{C}_{1}-\mathrm{C}_{2}\right)$ and $\pi^{*}(\mathrm{CH}=\mathrm{O})$ is about $16-20 \mathrm{~kJ} / \mathrm{mol}$. The hyperconjugative interaction between $\sigma\left(\mathrm{C}_{1}-\mathrm{C}_{2}\right)$ and $\pi *\left(\mathrm{CH}=\mathrm{CH}_{2}\right)$ is about $6-9 \mathrm{~kJ} / \mathrm{mol}$. The hyperconjugative interaction between $\sigma\left(\mathrm{C}_{1}-\mathrm{N}\right)$ and $\pi^{*}(\mathrm{CH}=\mathrm{O})$ is about $3 \mathrm{~kJ} / \mathrm{mol}$. The hyperconjugative interaction between $\sigma\left(\mathrm{C}_{1}-\mathrm{N}\right)$ and $\pi^{*}\left(\mathrm{CH}=\mathrm{CH}_{2}\right)$ is about $4-5 \mathrm{~kJ} / \mathrm{mol}$. 
Table 5. The hypercomjugative interaction energies in $\mathrm{C}_{1}$-substituted

2-aziridinylmethyl radicals $(\mathrm{kJ} / \mathrm{mol})$.

\begin{tabular}{ccccc}
\multicolumn{5}{c}{} \\
\multicolumn{5}{c}{} \\
\hline $\mathrm{R}_{2}$ & $\sigma\left(\mathrm{C}_{1}-\mathrm{C}_{2}\right)-\pi^{*}$ & $\sigma\left(\mathrm{C}_{1}-\mathrm{C}_{2}\right)-\sigma^{*}\left(\mathrm{C}_{2}-\mathrm{N}\right)$ & $\sigma\left(\mathrm{C}_{1}-\mathrm{N}\right)-\pi^{*}$ & $\sigma\left(\mathrm{C}_{1}-\mathrm{N}\right)-\sigma^{*}\left(\mathrm{C}_{2}-\mathrm{N}\right)$ \\
\hline cis $-\mathrm{CHO}$ & 16.1 & 2.2 & 3.2 & 9.2 \\
trans $-\mathrm{CHO}$ & 19.7 & 2.8 & 3.0 & 9.1 \\
cis $-\mathrm{CH}=\mathrm{CH}_{2}$ & 6.1 & 2.8 & 5.2 & 9.4 \\
trans $-\mathrm{CH}=\mathrm{CH}_{2}$ & 7.9 & 2.9 & 4.4 & 9.1 \\
$\mathrm{H}$ & - & 3.0 & - & 9.6 \\
cis $-\mathrm{CH}_{3}$ & - & 3.0 & - & 9.7 \\
trans $-\mathrm{CH}_{3}$ & - & 3.4 & - & 9.6 \\
cis $-\mathrm{NH}_{2}$ & - & 3.1 & - & 9.8 \\
trans $-\mathrm{NH}_{2}$ & - & 3.1 & - & 9.7 \\
\hline
\end{tabular}

It is worth noting that the $\sigma\left(\mathrm{C}_{1}-\mathrm{C}_{2}\right)$ and $\sigma\left(\mathrm{C}_{1}-\mathrm{N}\right)$ orbitals also interact with the antibonding $\sigma^{*}\left(\mathrm{C}_{2}-\mathrm{N}\right)$ orbital via hyperconjugation. Compared to the unsubstituted case, the hyperconjugative interaction between the $\pi^{*}$ orbital of the $\mathrm{CH}=\mathrm{O}$ or $\mathrm{CH}=\mathrm{CH}_{2}$ substituent and the $\sigma\left(\mathrm{C}_{1}-\mathrm{C}_{2}\right)$ or $\sigma\left(\mathrm{C}_{1}-\mathrm{N}\right)$ orbital should decrease the energy level of the $\sigma\left(\mathrm{C}_{1}-\mathrm{C}_{2}\right)$ or $\sigma\left(\mathrm{C}_{1}-\mathrm{N}\right)$ orbital. As a result, the $\sigma\left(\mathrm{C}_{1}-\mathrm{C}_{2}\right)-\sigma^{*}\left(\mathrm{C}_{2}-\mathrm{N}\right)$ and $\sigma\left(\mathrm{C}_{1}-\mathrm{N}\right)-\sigma^{*}\left(\mathrm{C}_{2}-\mathrm{N}\right)$ hyperconjugative interactions should be weaker in the $\mathrm{C}_{1}-\mathrm{CHO}$ and $\mathrm{C}_{1}-\mathrm{CH}=\mathrm{CH}_{2}$ cases than in the unsubstituted case. This prediction is confirmed by the NBO analysis. As shown in Table 5, the $\sigma\left(\mathrm{C}_{1}-\mathrm{C}_{2}\right)-\sigma *\left(\mathrm{C}_{2}-\mathrm{N}\right)$ interactions in the $\mathrm{C}_{1}-\mathrm{CHO}$ and $\mathrm{C}_{1}-\mathrm{CH}=\mathrm{CH}_{2}$ cases are $0.1-0.7 \mathrm{~kJ} / \mathrm{mol}$ weaker than that in the unsubstituted case. The $\sigma\left(\mathrm{C}_{1}-\mathrm{N}\right)-\sigma^{*}\left(\mathrm{C}_{2}-\mathrm{N}\right)$ interactions in the $\mathrm{C}_{1}-\mathrm{CHO}$ and $\mathrm{C}_{1}-\mathrm{CH}=\mathrm{CH}_{2}$ cases are 0.2-0.5 $\mathrm{kJ} / \mathrm{mol}$ weaker than that in the unsubstituted case. 
Since a weaker $\sigma\left(C_{1}-C_{2}\right)-\sigma^{*}\left(C_{2}-N\right)$ or $\sigma\left(C_{t}-N\right)-\sigma^{*}\left(C_{2}-N\right)$ hyperconjuation should increase the strength of the $\mathrm{C}_{2}-\mathrm{N}$ bond, it is now understandable that the $\mathrm{C}-\mathrm{N}$ cleavage activation free energy is increased by the $\pi$-acceptor $C_{1}$-substituents (including the groups with both $\pi$-acceptor and $\pi$-donor characters). On the other hand, the $\pi$-donor substituents such as $\mathrm{CH}_{3}$ and $\mathrm{NH}_{2}$ do not have the $\pi^{*}$ orbital and therefore, they cannot have the $\sigma\left(\mathrm{C}_{1}-\mathrm{C}_{2}\right)-\pi^{*}$ or $\sigma\left(\mathrm{C}_{1}-\mathrm{N}\right)-\pi^{*}$ hyperconjugative interaction. In consequence, the $\pi$-donor substituents cannot increase the $\mathrm{C}-\mathrm{N}$ cleavage activation free energy by weakening the $\sigma\left(\mathrm{C}_{1}-\mathrm{C}_{2}\right)-\sigma^{*}\left(\mathrm{C}_{2}-\mathrm{N}\right)$ or $\sigma\left(\mathrm{C}_{1}-\mathrm{N}\right)-\sigma^{*}\left(\mathrm{C}_{2}-\mathrm{N}\right)$ hyperconjuation. 